National Institute on Drug Abuse

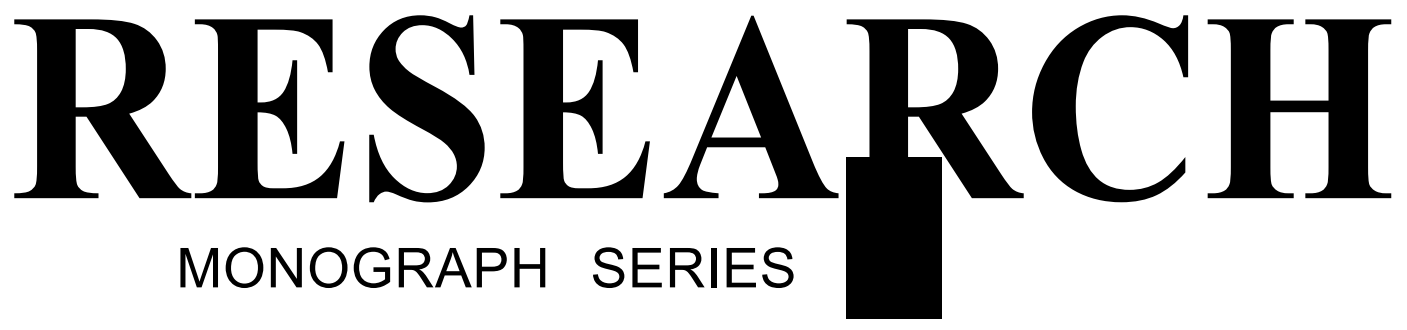

\title{
Problems of Drug
}

Dependence 1994:

Proceedings of the

\section{6th Annual Scientific}

Meeting

The College on Problems

of Drug Dependence, Inc.

\section{Volume I}




\section{Problems of Drug \\ Dependence, 1994:}

Proceedings of the 56th Annual

Scientific Meeting, The College on Problems of Drug Dependence, Inc.

\section{Volume I: Plenary Session Symposia and Annual Reports}

Editor:

Louis S. Harris, Ph.D.

NIDA Research Monograph 152 1995

U.S. DEPARTMENT OF HEALTH AND HUMAN SERVICES

Public Health Service

National Institutes of Health

National Institute on Drug Abuse

5600 Fishers Lane

Rockville, MD 20857 


\section{ACKNOWLEDGMENT}

The College on Problems of Drug Dependence, Inc., an independent, nonprofit organization, conducts drug testing and evaluations for academic institutions, government, and industry. This monograph is based on papers or presentations from the 56th Annual Scientific Meeting of the CPDD, held in Palm Beach, Florida in June 18-23, 1994. In the interest of rapid dissemination, it is published by the National Institute on Drug Abuse in its Research Monograph series as reviewed and submitted by the CPDD. Dr. Louis S. Harris, Department of Pharmacology and Toxicology, Virginia Commonwealth University was the editor of this monograph.

\section{COPYRIGHT STATUS}

The National Institute on Drug Abuse has obtained permission from the copyright holders to reproduce certain previously published material as noted in the text. Further reproduction of this copyrighted material is permitted only as part of a reprinting of the entire publication or chapter. For any other use, the copyright holder's permission is required. All other material in this volume except quoted passages from copyrighted sources is in the public domain and may be used or reproduced without permission form the Institute or the authors. Citation of the source is appreciated.

Opinions expressed in this volume are those of the authors and do not necessarily reflect the opinions or official policy of the National Institute on Drug Abuse or any other part of the Department of Health and Human Services.

The U.S. Government does not endorse or favor any specific commercial product or company. Trade, proprietary, or company names appearing in this publication are used only because they are considered essential in the context of the studies reported herein.

NIH Publication No. 95-3882

Printed 1995

NIDA Research Monographs are indexed in the Index Medicus. They are selectively included in the coverage of American Statistics Index, Biosciences Information Service, Chemical Abstracts, Current Contents, Psychological Abstracts, and Psychopharmacology Abstracts. 


\title{
BOARD OF DIRECTORS
}

George E. Bigelow, Ph.D., President

Edward Sellers, M.D., Ph.D., President-Elect

Thomas J. Crowley, M.D., Past-President

Joseph V. Brady, Ph.D., Treasurer

Robert L. Balster, Ph.D.

Edgar H. Brenner, JD

Lawrence S. Brown, Jr., M.D., MPH

Leonard Cook, Ph.D.

Linda A. Dykstra, Ph.D.

Charles W. Gorodetzky, M.D., Ph.D.

Roland R. Griffiths, Ph.D.

Jack Henningfield, Ph.D.
Stephen G. Holtzman, Ph.D.

Thomas R. Kosten, M.D.

Mary Jeanne Kreek, M.D.

Billy R. Martin, Ph.D.

A. Thomas McLellan, Ph.D.

David F. Musto, M.D.

Charles P. O'Brien, MD, Ph.D.

Roy W. Pickens, Ph.D.

Craig Reinarman, Ph.D.

James E. Smith, Ph.D.

Maxine L. Stitzer, Ph.D.

James H. Woods, Ph.D.

\section{EXECUTIVE OFFICER}

Martin W. Adler, Ph.D.

\section{SCIENTIFIC PROGRAM COMMITTEE}

\author{
Mary Jeanne Kreek, M.D., Chair \\ Martin W. Adler, Ph.D. \\ William L. Dewey, Ph.D. \\ Loretta P. Finnegan, M.D. \\ Ellen B. Geller, M.A. \\ Thomas Kosten, Ph.D. \\ Billy R. Martin, Ph.D. \\ Kenner C. Rice, Ph.D. \\ Eric J. Simon, Ph.D. \\ James E. Smith, Ph.D. \\ Maxine L. Stitzer, Ph.D.
}


The following organizations have generously supported the work of the College on Problems of Drug Dependence during the past year:

Berlex Labs, Inc.

Boehringer Ingelheim Pharmaceuticals, Inc.

Burroughs Wellcome Company

DuPont - Merck

Hoffman-La Roche, Inc.

Merck \& Co., Inc.

National Institute on Drug Abuse

Neurobiological Technologies

Parke Davis

Pfizer Inc.

Sandoz, Ltd. (Basle)

Schering-Plough, Inc.

Sterling - Winthrop

The Upjohn Company

Wyeth-Ayerst Laboratories

Zeneca 


\section{TABLE OF CONTENTS}

\section{PLENARY SESSION}

President's Comments: Behavioral Science is Fundamental to Drug Abuse Research and Treatment

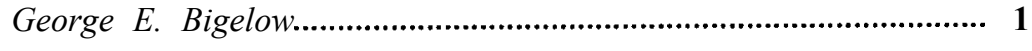

Drug Abuse and Addiction Research: Opportunities for Progress in 1995

Alan I. Leshner

Introduction to the Nathan B. Eddy Memorial Award Recipient J. V. Brady

The Nathan B. Eddy Lecture: Science, Policy, Happenstance

J. H. Jaffe

SYMPOSIUM I

The Relationship of Science to Drug Abuse Policy

Titles and Participants

Chairpersons: C. R. Schuster and S. W. Gust

Does Science Really Inform the Making of Substance Abuse Policy? H. Kleber

Formulating Drug Policy: A View from Congress R. H. Weich

Scientists and Policymakers: Strange Bedfellows

A. L. Solarz

Discussant

M. Kleiman

\section{SYMPOSIUM II}

Update on Opioid Receptor Cloning: Implications for Research on Addiction

Titles and Participants

Chairpersons: M. J. Kreek and E. Simon

Characterization of Opioid Receptors at the Molecular Level

C. Evans

Human Opiate Receptors and Their Genes

G. Uhl; J. Wang; P. Johnson; Y. Imai; D. Walther; J. M. Wu;

$W$. Wang; and A. Moriwaki

Opioid Receptors: Molecular Cloning and Cellular Function

Lei Yu 
Genomic Structure and Analysis of Promoter Sequence of a Mouse Mu Opioid Receptor Gene
B. H. Min; L. B. Augustin; R. F. Felsheim; J. A. Fuchs: and H. Loh

Mapping and Modulation of Opioid Receptor Gene Expression M. J. Kreek

Quantitation and Localization of Opioid Receptor Gene Expression C. Inturrisi

Historical Summary of NIDA Supported Opioid Research: A Tribute to NIDA on its 20th Anniversary

E. J. Simon.

SYMPOSIUM III

Drug Abuse and the Health of Women .45

Titles and Participants

Chairpersons: L P. Finnegan and M. J. Kreek

Epidemiology of Drug Abuse Among Women Z. Sloboda

Infectious Disorders in Drug Abusing Women H. Haverkos

Endocrine Changes and Drug Abuse in Women M. J. Kreek and N. Mello

Psychopathology, Life Events and Treatment - History of Female Drug Abusers

L. B. Cottler

The Impact of Drug Abuse on Children D. Frank

SYMPOSIUM IV

Affective Disorders and Substance Abuse: From Bench to Bedside and Back

Titles and Participants

Chairpersons: T. R. Kosten

Common Genetic Mechanisms in Depression and Substance Abuse R. W. Pickens

Clinical Studies on the Relationship of Affective Disorders to Substance Abuse

T. R. Kosten

Co-vulnerability Between Affective Disorders and Substance Abuse: Preclinical Perspectives

E. Gardner 
Post-Drug Elevations in Reward Thresholds as an Animal Model of Affective Disorders

A. Markou and G. Koob

SYMPOSIUM V

Young Investigator's Symposium

Environmental Modulation of Drug Effects

Titles and Participants

Chairpersons: J. Kamien and L. Amass

Environmental Determinants of the Neurochemical Effects of Drugs S. L. Vrana

Environmental Modulation of the Discriminative Stimulus Effects of Drugs

J. B. Kamien

Environmental Modulation of the Reinforcing Effects of Drugs M. A. Nader

Environmental Modulation of the Subject-Rated Effects of Drugs C. R. Rush

Environmental Modulation of Drug Effects in Drug Abuse Treatment L. Amass

SYMPOSIUM VI

Brain Imaging in Substance Abuse...........................................................58

Titles and Participants

Chairpersons: C. P. O'Brien and A. R. Childress

Brain Metabolism in Substance Abuse

E. London

Fluoro-dopa Studies of Human and Animal Cocaine Abuse

L. Baxter, Jr.

EEG Brain Mapping of Drug-Induced Intoxication

S. Lukas

Dopaminergic Involvement in Cocaine Dependence

N. Volkow

SPECT Imaging of DA Transporters in Cocaine Dependence with $\left[{ }^{123} 1\right]$-CIT

R. Malison

Brain Imaging During Drug-Craving States

A. R. Childress

Discussant

C. P. O'Brien 
Titles and Participants

Chairpersons: A. H. Newman and K. C. Rice

In Memoriam

P. S. Anderson

A Tribute to Dr. Frank Robinson

K. C. Rice

Interaction of Nonpeptidal Ligands at Opioid Receptor Sites P. Portoghese

Development of Selective Nonpeptide Ligands for Further Characterization of Delta Opioid Receptors

$S$. Calderon

Opioids - From Analgesics to Addict Treatments

J. Lewis

Substituted 3-Carbamoylecgonine Methyl Ester Analogues: Novel Probes for the Cocaine Recognition Site on the Central Dopamine Transporter R. Kline

Studies of Conformationally Constrained Phenethylamines

P. Anderson

Novel 30.-Diphenylmethoxytropane Analogs are Potent Dopamine Uptake Inhibitors Without a Cocaine-Like Behavioral Profile

A. H. Newman

\section{SYMPOSIUM VIII}

Is There a Dose-Response Curve for Psychosocial Services?.....................71

Titles and Participants

Chairpersons: J. A. Hoffman and B. W. Fletcher

Effects of Amount of Services on Outcomes During Treatment:

Preliminary Analysis from Two National Studies of Community-Based

Treatment Programs

R. L. Hubbard; R. M. Etheridge; S. G. Craddock; and

G. Dunteman

Psychosocial Treatments for Cocaine Abuse: Treatment Intensity as a Predictor of Long-Term Clinical Outcomes

J. A. Hoffman; B. D. Caudill; J. Koman, III; J. W. Lucke;

P. M. Flynn; and D. W. Mayo

Dose-Response Studies of Psychosocial Services During Substance

Abuse Treatment

A. T. McLellan 
Etiology, Epidemiology and Natural History of Heroin Addiction:

A Social Science Approach

Titles and Participants

Chairpersons: J. C. Ball and D. N. Nurco

Sociology of Drug Abuse in Contemporary America R. R. Clayton

Social Pathology and the Onset of Heroin Addiction D. N. Nurco

Special Treatment Needs of Female Opiate Addicts

T. A. Hagan

Characteristics of Patients in Methadone Maintenance Programs in the United States

J. C. Ball and G. A. Groves

SYMPOSIUM $X$

Buprenorphine: Current Status for the Treatment of Opioid

Dependence

Titles and Participants

Chairpersons: W. K. Bickel and M. L Stitzer

Clinical Pharmacology of Buprenorphine M. L. Stitzer

Buprenorphine as a Detoxification Agent R. E. Johnson

Routes of Prior Opiate Administration: Effects on Outcome Variables in the NIDA/MDD \#999a Buprenorphine Multicenter Study D. L. Segal and J. L. Hill

Improving Buprenorphine Outcomes with Behavioral Treatment W. K. Bickel, L. Amass, and S. T. Higgins

\section{ANNUAL REPORTS}

Biological Evaluation of Compounds for Their Physical Dependence Potential and Abuse Liability. XVIII. Drug Evaluation Committee of the College on Problems of Drug Dependence, Inc. (1994)

A. E. Jacobson

Progress Report from the Testing Program for Stimulant and Depressant Drugs (1994)

G. Winger; L. Woolverton; J. K. Rowlett; J. A. English;

G. A. Patrick; M. A. Nader; R. E. McDaniel; W. T. Hawkins;

B. W. Massey; L. S. Harris; and J. H. Woods 
Evaluation of New Compounds for Opioid Activity (1994)

J. H. Woods; F. Medzihradsky; C. B. Smith; C. P. France; and $G$. Winger

Dependence Studies of New Compounds in the Rhesus Monkey, Rat and Mouse (1994)

M. D. Aceto; E. R. Bowman; L. S. Harris; and E. L. May........... 162

Subject Index

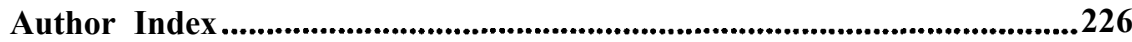




\title{
PRESIDENT'S COMMENTS: BEHAVIORAL SCIENCE IS FUNDAMENTAL TO DRUG ABUSE RESEARCH AND TREATMENT
}

\author{
George E. Bigelow
}

It is an honor to have this opportunity, as president of the College, to offer some personal comments relevant to the drug abuse field. The theme I have chosen to emphasize is that behavioral science methods arc of great importance and value -indeed fundamenral -- to both the scientific study of, and the practical treatment of drug abuse.

This theme is important for two reasons -- one a congratulatory reason related to the past, the other a cautionary reason related to the future. First, the congratulatory and historical reason: Throughout NIDA's 20-year history there has been a strong and mutually beneficial relationship between the Institute and the behavioral sciences. Behavioral science research has guided and transformed our conceptualization of the nature of drug abuse and has contributed to major scientific advances in both the laboratory and the clinic. NIDA has served as a model Institute for demonstrating how behavioral sciences can be effectively represented and integrated with more fundamental biological sciences to sustain a broad and balanced research program addressing all aspects of the drug abuse problem. NIDA is to be congratulated for this continuing success. The second reason for emphasizing this behavioral sciences theme is the cautionary one regarding the future: NIDA is undergoing a period of transition -- with a new

Director and administrative transfer into NIH -- and special vigilance and efforts may be needed to sustain into the future NIDA's successful broad-based, and balanced research program. Specifically, there is reason for concern that the behavioral science components of NIDA's research program may suffer as a consequence of the transfer to $\mathrm{NIH}$, where there is a strong molecular biology zeitgeist and where behavioral sciences have traditionally been poorly represented despite repeated congressional mandates to provide greater emphasis. Ideally, NIDA, NIAAA, and NIMH would serve as models for the rest of NIH about how effectively to integrate behavioral sciences so as to sustain broad-based and balanced research programs. However, as the grant review of these institutes is transferred to NIH special effort and caution will likely be needed if the important contributions of the behavioral sciences are to be preserved.

The sections below briefly address the following points regarding the role of behavioral sciences in drug abuse research and treatment: First, that drug abuse is a behavioral disorder for which the critical endpoints of interest are behavioral; second, that drug self-administration is biologically normal; third, that drugtaking is learned behavior and is controlled by the normal principles of learning; fourth, that there are excellent behavioral models of drug abuse available in both the animal laboratory and the human laboratory; fifth, that behavioral-sciencederived treatments arc effective, and appear to have a cross-drug generality that is lacking in more pharmacologically- or biologically-based treatments. 


\section{BEHAVIORAL DISORDER}

It should be self-evident that drug abuse is a behavioral disorder. The core defining feature of the disorder is the behavior of drug self-administration, and the critical endpoints of interest at both the individual level and the societal level are behaviors. Drug self-administration itself is not the only behavioral endpoint of interest; drug-seeking behavior is also of major interest, since it often appears as criminal activity. One of the especially interesting relationships revealed by behavioral science research is that these two behavioral endpoints may be affected in opposite directions by the same manipulations. This is illustrated by the effect of variations in the ease of drug availability (or cost) on drug use versus drug seeking behavior. Making drugs more difficult to obtain or more costly can decrease use at the same time that it increases drug-seeking-related criminal activity. This adverse behavioral effect of supply-reduction strategies is one of the strong empirical bases for advocacy of demand-reduction strategies as preferable.

\section{BIOLOGICAL NORMALITY}

The development of laboratory animal models of drug self-administration three to four decades ago has transformed scientific conceptualization of the nature of drug abuse. The discovery that normal healthy laboratory animals will selfadminister the same drugs that humans abuse has made evident the biological normality of drug reinforcement and drug abuse. No longer is it necessary to postulate brain disease, psychiatric illness, or sociologic disadvantage as a necessary foundation for drug abuse; these are now seen as being neither necessary nor sufficient. Rather, it is recognized that drugs of abuse have CNS effects in normal individuals that, in appropriate behavioral circumstances, can lead to these drugs' functioning as reinforcers and coming to control behavior.

I have recently been reading the popular non-fiction best-seller "Listening lo Prozac" by psychiatrist Peter Kramer, about the behaviorally transforming effects of psychotherapeutic medications, in this case fluoxetine, or PROZAC. One of the messages that Kramer is surprised to learn by listening to PROZAC -i.e., by observing the effects of fluoxetine in patients -- is that even individuals without significant disorders, normal individuals, can be behaviorally transformed by the medication. I am not as surprised as Kramer is; his realization seems analogous to what we have learned in the drug abuse field. Even normal individuals are vulnerable to the behaviorally transforming effect of drug reinforcement.

\section{LEARNED BEHAVIOR}

Behavioral science has not only shown drug reinforcement to be biologically normal, but has identified the mechanism of acquisition of drug selfadministration to be the normal processes of learning that are relevant to acquisition of other learned behaviors. That is, the mechanism of the behaviorally transforming effect of drug reinforcement is learning. Extensive research has further documented the substantial commonalities between animals and humans with respect to the controlling variables of this learned drug-taking behavior (Griffiths et al., 1980). 


\section{BEHAVIORAL MODELS}

One of the great strengths of the drug abuse field is that it has excellent experimental behavioral models of drug abuse that can be used to study the phenomenon and to examine factors that influence it. These arc available in both the animal laboratory and the human laboratory. Procedures such as assessing the profile of subjective and behavioral effects of drugs. drug discrimination, and drug self-administration have proven remarkably useful as research tools, making it possible to bring into the laboratory the critical defining elements of drug abuse and to examine directly the effects of variables that can be manipulated. Integration of these methods with more basic neuropharmacological information has permitted characterization of the specific pharmacodynamic effects associated with different profiles of neuroreceptor binding (Preston and Bigelow, in press).

These behavioral models have now been productively adapted and directed toward use in medications development research. In this research the goal is to identify medications that will reduce the drug-abuse-related characteristics of drugs of abuse.

This use of these laboratory behavioral models is illustrated by research related to the development of buprenorphine as a treatment for opioid abuse and dependence. A study in our laboratory examined the ability of buprenorphine to attenuate the subjective, behavioral and physiological effects of opioid challenge injections (Bickel et al., 1988). The maintenance dose of buprenorphine was varied and response to opioid challenge infections was assessed at each buprenorphine dose level. The results showed an orderly buprenorphine-doserelated decline in opioid challenge response. Similarly, Mello and Mendelson (1980) used a human laboratory opioid self-administration model and directly demonstrated that buprenorphine reduced opioid self-administration. These laboratory studies were critical steps in the developmental sequence for buprenorphine as a drug abuse treatment medication, and they provided the empirical basis for selection of the dosages to be used in subsequent multi-site outpatient clinical trials.

These human laboratory-model methods have now been adapted by several laboratories, including our own, to screen for compounds that may useful in treatment of cocaine abuse (Bigelow, 1993; Walsh et al., in press).

\section{THERAPEUTIC EFFICACY}

Drug abuse treatment procedures directly derived from behavioral science data and conceptualizations of drug abuse have repeatedly been shown to have significant therapeutic efficacy in outpatient clinical trials of drug abuse treatment. One of the basic principles of behavioral science is that if one wishes to reduce the frequency of some behavior it is most effective to reinforce some alternative, incompatible behavior. One such approach has been to offer incentives or reinforcers contingent upon providing drug-free urinalysis samples. There have now been numerous demonstrations that such positive reinforcement approaches to promoting drug abstinence are effective. These methods were pioneered by Stitzer at our laboratory at Johns Hopkins (e.g., Stitzer et al., 1992), and suhsequently elaborated and more fully developed by Higgins at the University of Vermont. For example, in one of Higgins' studies 
cocaine abusers received either a standard abstinence counseling treatment based on the traditional AA 12-step model, or a behaviorally-based contingent reinforcement treatment in which vouchers exchangeable for goods or services were earned contingent upon providing drug-free urinalysis results. Outcomes were dramatically superior in the behavioral treatment condition (Higgins et al., 1991)

One of the especially attractive features of these behavioral treatment methods is that they appear to have broad applicability to abuse of different drug classes. That is, methods developed and used to treat one type of drug abuse (e.g., opioid abuse) may be readily transferable and efficacious in the treatment of another type of drug abuse (e.g., cocaine abuse). This is in marked contrast to the very narrow pharmacological specificity that typically characterizes the efficacy of biological or pharmacological treatments.

A final point to be made regarding efficacy is that integration of behavioral and pharmacological treatments may be more effective than either alone, and in some cases such integration is essential for the pharmacological treatment to show any efficacy at all. For example, naltrexone treatment for opioid abuse and disulfiram (Antabuse) treatment for alcoholism have non-significant clinical efficacy when the treatment is provided as a routine medication prescription. However, when these medications arc combined with behavioral interventions -especially interventions designed to maintain medication ingestion -- these treatments can be remarkably effective. Even in the case of methadone treatment, which has substantial pharmacological efficacy (Strain et al., 1993), treatment outcomes depend critically upon the intensity of concurrent behavioral and psychosocial intervention provided (McLellan et al., 1993).

\section{CONCLUDING REMARKS}

In extolling the value, importance and contributions of behavioral science to the drug abuse field, I do not in any way minimize the value, importance and contributions of other research approaches. Drug abuse is a broad multidisciplinary field, and we should be committed to sustaining and promoting strong science throughout all of the diverse aspects and domains of the field. Science has two general goals that must be pursued simultaneously. One is to gain fundamental knowledge. The other is to yield practical benefits. Especially in the drug abuse field publicly-supported researchers are called upon to produce practical benefits to society. Practical benefits require appropriate technology. Appropriate technology is technology that is both effective and feasible within the system with which one has to deal. In drug abuse, the important individual and public health outcomes are at the level of complex behavioral systems and we must rely upon a substantial degree of compliance and individual acceptability in order to implement interventions. I would argue that in this context behavioral science or behavioral technology is appropriate technology. While we must concurrently pursue all levels of technology and analysis to answer basic scientific questions about drug abuse, we must continue to preserve and expand our behavioral science knowledge base in order best to achieve our practical public health and therapeutic goals in drug abuse. 


\section{REFERENCES}

Bickel, W.K.; Stitzer, M.L.; Bigelow, G.E.; Liebson, I.A.; Jasinski, D.R.; and Johnson, R.E. Buprenorphine: Dose-related blockade of opioid challenge effects in opioid dependent humans. J Pharmacol Exp Ther 247:47-53, 1988.

Bigelow, G.E. The role of human laboratory studies in drug abuse medications development. In Harris, L.S., ed. Problems of Drug Dependence, 1992, NIDA Research Monograph Series No. 132, 1993, pp. 38.

Griffiths, R.R.; Bigelow, G.E.; and Henningfield, J.E. Similarities in animal and human drug taking behavior. In: Mello, N.K., ed. Advances in Substance Abuse: Behavioral and Biological Research. Greenwich, CT: JAI Press, 1980, pp. 1-90.

Higgins, S.T.; Delaney, D.D.; Budney, A.J.; Bickel, W.K.; Hughes, J.R.; Foerg, F.; and Fenwick, J.W. A behavioral approach to achieving initial cocaine abstinence. Am J Psychiatry 148:1218-1224, 1991.

Kramer, P.D. Listening to Prozac. New York: Viking, 1993.

McLellan, A.T.; Arndt, I.O.; Metzger, D.S.; Woods, G.E.; and O’Brien, C.P. The effects of psychosocial treatment services in substance abuse treatment. JAMA 269:1953-1959, 1993.

Mello, N.K. and Mendelson, J.H. Buprenorphine suppresses heroin use by heroin addicts. Science, 207:657-659, 1980.

Preston, K.L. and Bigelow, G.E. Drug discrimination assessment of agonistantagonist opioids in humans: A three-choice saline-hydromorphonebutorphanol procedure. J Pharmacol Exp Ther in press.

Stitzer, M.L.; Iguchi, M.Y.; and Felch, L.J. Contingent take-home incentive: Effects on drug use of methadone maintenance patients. J Consult Clin Psychol 60:927-934, 1992.

Strain, E.C.; Stitzer, M.L.; Liebson, I.A.; and Bigelow, G.E. Dose-response effects of methadone in the treatment of opioid dependence. Ann Intern Med 119:23-27, 1993

Walsh, S.L.; Preston, K.L.; Sullivan, J.T.; Fromme, R.; and Bigelow, G.E. Fluoxetine alters the effects of intravenous cocaine in humans. J Clin Psychoipharmacol in press.

\section{AFFILIATION}

Behavioral Pharmacology Research Unit Johns Hopkins University School of Medicine

Mailing Address: BPRU, Behavioral Biology Research Center; Johns Hopkins Bayview Campus; 5510 Nathan Shock Drive; Baltimore, MD 21224-6823 


\title{
DRUG ABUSE AND ADDICTION RESEARCH: OPPORTUNITIES FOR PROGRESS IN 1995
}

\author{
Alan I. Leshner
}

I am extremely pleased to be here representing the National Institute on Drug Abuse (NIDA) as its new Director and to share with you my sense of excitement about the boundless opportunities for progress that await us in the drug abuse and addiction research field.

This year, as most of you know, marks the Institute's 20th anniversary -- two decades of progress in drug abuse research supported through NIDA. To commemorate the many remarkable achievements that have been made in the field we have planned a number of activities. These include a satellite symposium, cosponsored by NIDA and the National Foundation for Brain Research, to be held later this month at C.I.N.P. which will highlight some of the field's most notable advances in neuroscience and a day-long symposium to be held at NIH in the fall covering the full breadth of NIDA's research. Since many of you in this audience have played such a critical role in the accomplishments we celebrate, we hope that you can participate in these events and garner some of the recognition you so richly deserve.

From my perspective, we in the drug abuse arena are faced with the most complex, and probably the most alarming social and public health issue confronting this country. And, at the same time, we are faced with the most stigmatized public health issue that there is. We hide our drug treatment centers so no one knows what they are. We never enlist addicts to help us advocate. It is a terribly stigmatized disease and a terribly stigmatized set of problems and scientific issues which clearly makes our job all the more difficult.

Because of the magnitude of the problems, and because of the social concern that surrounds drug abuse and addiction, I believe that MDA must evolve itself into what I've been calling a prototypical public health institute. This is more than just the scientific establishment that carries out activities because they are interesting and potentially useful. In my view, a prototypical public health institute is characterized not only by superb science, but by the systematic interactive set of activities it engages in to reach out to the public; to the practice community; to the consumer and the advocacy communities in an effort to increase their understanding of and appreciation for what we do; and to help us in shaping our research agenda. Faced with a public health problem like drug abuse and addiction, it is not responsible to shape our research agenda in a vacuum. And therefore, over time, we will increase our interaction with the community, and will engage in more dialogue with policy makers to try to ensure that the questions that we as an Institute are asking are the questions that in fact are of paramount importance in the minds of these groups.

Since the College on Problems of Drug Dependence comprises most of the key figures in drug abuse research, I feel it is important for me, as NIDA's new director, to describe for you my philosophical position with regard to an Institute like NIDA and how it administers science. While at the National Science 
Foundation and then at the National Institute of Mental Health, I was often called upon to articulate, for a wide range of communities, my views on the federal role in the support of science. In an idealized view of how science, technology and product development or service delivery progresses, science informs technology which, in turn, informs product development. However, as we all know, this concept does not always match up with reality. Technology at the moment is driving science far more than science is driving technology. The molecular genetics revolution, the imaging revolution, the computer and information science revolutions are all changing the way in which we engage in scientific investigation far more than science is changing technology. In the case of product development in our fields, it has often been the discovery of products that drives science and technology far more than in the other direction. Nonetheless, the process, in general, is truly an interactive one.

There is also an idealized view in which basic research informs clinical research, which, in turn, informs applied or services research. In practice, however, the process is far more complex than this, and no one does any of these things in a vacuum. The truth is that progress in a particular area is an interactive process. Because it's an interactive process, we as a scientific institute have an obligation to be listening to the world as much as we are telling the world. Although this concept is easy to articulate, it is an extremely difficult thing to operationalize and something NIDA will be working on over the next few years in a very systematic way. If we want to have an impact, it is imperative that we find ways to enhance our interactions and our dialogue with our constituency groups.

The third philosophical point I would like to address involves the way in which we relate to the scientific community. If we characterize the various ways the federal government supports science as being along a continuum, on one end would be a totally reactive approach. That is, the products coming in are reviewed and then the best are supported. This strategy, also known as the thousand flowers, field generated, or reactive approach is what was said to be true in the good old days.

And then we went through a period characterized by the Sputnik event that shifted the Federal Government's approach to the other end of the continuum which was putting a man on the moon -- a very directive, targeted, pro-active tactic which is viewed as the other end of the continuum. That era came to be known as the bad new days because the scientific community felt that they were essentially being directed by the Federal Government.

In truth, this continuum varies by agency or institute, and it varies according to the purpose of the scientific enterprise. And it tracks with whether we as a Federal Government are at that point in history supporting science for its own sake, for knowledge's sake, or whether we do it because it is meeting a specific set of national needs.

As a public health institute NIDA probably stands somewhere in the middle of this continuum. That is, we are not able to or interested in just propagating a thousand flowers -- let the world send us whatever there is. Employing this approach, science often does not progress as rapidly as it might with at least a somewhat targeted strategy. At the other extreme, there are few problems for which a totally strategic, targeted approach has been taken, and too much targeting has not been helpful as an overall strategy.

So for NIDA I think we need to determine where the frontier is and where the 
cutting edge is and move in that direction on the continua, about in the middle. To illustrate my views, I find the metaphor of a hockey game particularly appropriate. As Wayne Gretzky has said, "I skate to where the puck is going to be." What we need the drug abuse research community to do is to help us identify where the puck is going to be and determine the best way to get there. This is what we will be soliciting your input on in the coming months.

In carrying out this metaphor, skating translates into setting our priorities, our emphases, and our initiatives. Our task is to try to help to focus the research that we support in particular areas. What we will be asking of you as knowledgeable members of the drug abuse research community is to help us skate in the right direction.

In order to lay out a plan for addressing the problems of drug abuse and addiction, I think it is important for me to spend a little bit of time talking about a conceptualization of this complex disease. First of all, I consider it extremely important to recognize drug abuse and addiction as both a social and a public health issue. And we must focus our research efforts on both aspects. I would also like to make the point that drug addiction itself, once it occurs, is a brain disease; it is an event that modifies the brain in some way that is expressed in behavioral ways and in a social context. And when you conceptualize drug abuse and addiction in these ways, you see it not only as among the most complex, but the most difficult kinds of issues that you could try to combat. Because of its complexity, the study of drug abuse and addiction requires that we take a multiple discipline attack.

What we need to do is to bring the multiple levels of analysis and the tools of multiple disciplines to bear on this problem. NIDA historically has taken this type of broad-based approach, from the molecular level all the way to the level of studying political systems and policy systems, and service systems, and we will continue to do that as well.

Since my arrival at NIDA about three and a-half months ago, we have been trying to articulate some of the major initiative areas and some of the major priorities that NIDA is going to take on in the near term. These have included the programs that are already ongoing as well as activities that we have in the mill. I would like to go through these sequentially and give you some very broad descriptions of what we've triggered internally and, hopefully, research areas that you will help us shape.

\section{TREATMENT DEVELOPMENT}

Historically, drug abuse and addiction treatment has been directed in two ways at NIDA and we will continue to do that, focusing both on the development of medications and on more traditional behavioral and psychosocial treatment. I subscribe to the view that, in many cases, the most effective treatment ultimately will be a combination of these. If you view addiction as a brain disease that is expressed in behavioral ways and in a social context, you cannot use only biological treatment, nor can you use only behavioral treatment. Increasingly, we are learning how behavioral treatment can modify the brain in addition to changes in the brain having the capability of altering behavior.

In medications development, we take basically two kinds of approaches. One I call opportunistic, the other is strategic. Although the opportunistic approach has actually been highly productive in many fields, it has not given us too many 
medications to treat drug abuse and addiction so far. The second approach is a more strategic one, to use the science that we generate as a base from which we then proceed to develop new medications. That is, applying medicinal chemistry and other similar techniques to capitalize on neuroscience advances. The area that I think we are most hopeful about and that our Medications Development Division has been working diligently on is, taking advantage of the discovery that cocaine works via the dopamine re-uptake transporter, searching for a dopamine sparing cocaine blocker. This is a far more strategic approach to developing effective treatments than we have been able to take in the past. Over time, we will try to become more and more strategic so that the small amount of funds we do have available is employed in the most leveraged way that is possible.

NIDA's treatment improvement program also includes the area of behavioral or psychosocial treatment. My limited experience suggests to me that much of what we currently support in this area is incremental research. There are a range of extant behavioral treatments that we have been exploring but, to date, we have not really devoted a great deal of our energy or resources in search of truly new or novel approaches. We will, of course, continue to support research to provide incremental improvements on treatments currently available and to understand what can make those treatments work better and for whom. But at the same time, I believe that we need to expand our horizons and start looking for truly novel approaches to treatment as well.

To facilitate this process we have begun to talk inside the Institute about convening a meeting of treatment researchers from within the drug abuse field and treatment researchers from outside the drug abuse field, to brainstorm about approaches that have proven to be effective in other areas of scientific investigation which might be applied to drug abuse and addiction. I believe that if we want to achieve a measurable increment in drug treatment in this country, we are going to have to take some novel looks at ways in which to approach treatment. As a scientific institute, we will always do that based on science. We will do it based on theory. We will do it based on findings that have occurred in a variety of fields. But we have to start looking at some novel approaches.

\section{NEUROSCIENCE}

Neuroscience is an area in which NIDA has made remarkable advances over the course of the last few years. There are a variety of activities that we are currently involved in as well as others we hope to be initiating soon.

First of all, soon after I arrived at NIDA, we established an Institute-wide group we have called NIDA's Neuroscience Consortium. Formerly our Neuroscience Program Management Committee, the name of this group was modified not only to reflect its importance but also the fact that we want the activities the group becomes involved in to span the entire range of what NIDA supports -- basic science, clinical science, and, where possible, applied science as well. Cochaired by Drs. Christine Hartel and Karen Skinner, the group is currently in the process of developing a strategy and a set of steps we should take to advance what we do in the neuroscience arena.

The long standing Clinical Human Neuroscience Initiative NIDA has been supporting is another example of the Institute's commitment to broadening our neuroscience program. I personally view this as an exciting and necessary area to explore. Thus far, we have received numerous excellent proposals and we are very hopeful that as these projects are reviewed we can make some significant 
awards in the near term.

The Human Brain Project is another activity you may have heard about on numerous occasions as something NIDA is centrally interested in. I am proud to be among this project's fathers, and I believe that the information that will result is a significant contribution that the Federal Government will make to the establishment of an infrastructure for neuroscience research. For this reason, I think every neuroscience institute has an obligation to be a part of it. NIDA will continue to play an active role in this important activity.

The progress made possible by the technological advances of the past decade are particularly extraordinary. It is truly astonishing that over the past 20 years, the drug abuse field has accomplished the task of cloning one or more receptors in the brain for every major drug of abuse. This is an achievement that the drug abuse field alone can purport and one whose implications for research in the field are enormous. Building on these advances, NIDA will move beyond cloning, using the technology currently available to pursue this basic cloning phenomenon both from a neuroscience perspective as well as from a medications development perspective.

\section{$\underline{\text { AIDS }}$}

HIV infection and AIDS continue to take an enormous toll on the health of our nation. Drug related behaviors such as sharing of contaminated injection equipment or having sex with an injecting drug user are the second leading cause of HIV infection and AIDS, accounting for more than one-third of cases reported. Our efforts to bring drug using individuals into treatment are the first and most effective line of defense. But for those who continue to use drugs, modifying the behaviors that place them at risk can significantly reduce their chances of contracting this devastating disease. For over a decade, NIDA's efforts at devising effective behavior change strategies have helped to curtail the potential magnitude of the problem. But much still remains to be done. Clearly, our AIDS program needs far more rationalization. In the months to come, we will be spending more time working on our portfolio and plans for this area. AIDS related research represents one-third of our budget and we need to be very systematic about what we are doing and what more we need to do in this arena.

\section{HEALTH SERVICES RESEARCH}

Another area where NIDA has made a great deal of progress recently is services research. This field represents a major opportunity and one for which it is vital that we play an active role now. Although we may not have articulated the extent of our involvement as best we might, NIDA has actually been at the forefront of the activity that has recently been taking place. There has been one issue on the table which has been of paramount importance in the public health arena in 1994, and that issue is health care reform. If we want to be credible players in the process, if we want to make sure that the people we serve -- people with drug abuse and addiction problems in this country -- are fairly treated; we must be sure that we have a knowledge base that we can contribute about the effectiveness of treatment and about cost effective ways to organize and finance care. Although this type of knowledge base is a critical need in all areas of public health, throughout much of the debate over health care reform, the addictive and mental disorders have been experiencing a relatively more difficult time in ensuring their inclusion in the health care legislation being proposed. We are constantly being asked for data about the effectiveness of treatment, whether or not it works, who 
does it work for, is it cost effective, and what does it cost. It is the services research base that will provide the information to answer these and a host of other policy related questions.

What NIDA intends to do is to move from the live year planning process that has been under development and devise a strategic plan for services research that will involve a large number of people in both the policy and practice communities, as well as in the scientific community. It is my hope that many of you will help us determine what questions need to be asked, and what issues need to be addressed in order to best inform policy and practice. Over time, we intend to increase our efforts to generate scientific information to bring to bear on these issues.

\section{CLINICAL RESEARCH INFRASTRUCTURE}

Tied to our need for a variety of approaches to drug abuse treatment is the concomitant problem of having far too sparse a clinical research infrastructure for drug abuse in this country. It is clear that our clinical research infrastructure is inadequate. There is an urgent need to attract more clinical researchers to the drug abuse and addiction field and to expand our overall capability to support their efforts. We will accomplish these objectives in a variety of ways, focusing both on more researchers and on more institutions.

\section{RESEARCH INFRASTRUCTURE}

In addition to the clinical research infrastructure inadequacies that exist in the drug abuse field, research infrastructure in general is also relatively deficient. That is, we have far too few researchers in our field. This is a smaller field than it ought to be, given the magnitude of the problem, and given the public interest it receives. In order to actually accomplish that multiple discipline strategic attack on drug abuse and addiction that is necessitated by the problem's complexity, we are going to have to be systematic about the way in which we increase the size and the scope of the field. And again, we will have to do it both institutionally and in terms of individual investigators.

Within the NIH structure we support a number of mechanisms which provide opportunities for strengthening the field's infrastructure including First Grants -the awards for young investigators, small grants and research training. Over time, you will be seeing increased attention to these kinds of activities. It is imperative that we develop seed corn for the future in order for the field to continue to flourish.

It is my firm belief that the best way to recruit people into a particular scientific field is through mentoring. I would challenge anyone to tell me how you could conceivably get interested in an area of research if you never met anyone who did that kind of research. A study was done many years ago of how Nobel Laureates had come into science. And every single one of them had done it by a hands-on research experience with someone they bonded to. In order to facilitate this process we need to find ways to provide resources to the scientific leaders in our field to enable them to function as mentors. This, I would argue, is a complementary strategy to developing seed corn to try to bring additional people into the field. Thus, NIDA will also be developing opportunities in each of these areas.

In addition to needing more researchers, we also need to make sure that we are making the best use of the mechanisms we have available. Among the things I've 
been concerned about since coming to NIDA, is to ensure that the Institute's Research Centers program is organized in as strategic a way as possible. We have, therefore, begun discussions to try to rationalize and develop our Research Centers program.

This is a very important issue, because the way you conceptualize research centers determines both what you are trying to accomplish by establishing them as well as the way in which you structure support for them. In my view, research centers fall into two groups -- those that I would term enabling research centers and those that are stimulating research centers.

The stimulating research centers are relatively easy to conceptualize. In an area where research is sorely needed but is not being conducted you might infuse some money to initially set up a program. This was the technique used, incredibly effectively, when the AIDS problem began. The Public Health Service established a large number of AIDS centers initially to stimulate research on AIDS. As more and more researchers came into the AIDS field, the centers became a much smaller part of the total AIDS budget and there were more and more R01s being supported, but it was a way to draw people into the field. This method has been used effectively in services research, in prevention research, and a whole variety of other areas. It is a very useful technique and a very important one in stimulating research in an area where it is needed. In designing our portfolio we may need to create centers for this purpose.

However, I believe that most of the centers we support should be of the other category which I call enabling research. Why do you want a center? You want a center in order to do something that you couldn't do if you just had an R01. There is no point in having a center if it is merely an aggregation of R01 applications. In this case, each of the projects gets reviewed individually and instead of going through five committees, the projects go through one committee as one large integrated project. From NIDA's point of view, all that does is eat R01s. This is not a very effective way of supporting centers in the majority of cases.

What we do want is to provide support for research that could not be done without a center vehicle. And clinical research is very often an example of that. For example, if you don't have the infrastructure present, if you don't have a subject population, if you don't have beds, if you don't have the kind of core support that you need to do clinical research, it often is very difficult to do that research. It also is true in cases of shared equipment. One of the biggest things that has come along and is providing tremendous opportunities for progress in our field is the neuro-imaging revolution. I believe that neuro-imaging will revolutionize our understanding of the brain. However, we are finding it difficult to get any time on existing machines. And if you can't get time on machines and you have to line people up four weeks in advance -- a little hard with people who are addicted to drugs -- then the fact that the technology exists does you very little good. We need to create mechanisms to enable the research we want done to be done.

Over time, we will try to rationalize our Research Centers programs so that the centers that we establish are designed to accomplish specific goals. Our job is to then provide the resources that are necessary to accomplish the goal. If you view our task, as I do, as facilitating science or enabling the science to be done, we must not only provide the resources necessary to conduct the work, but we must do it in such a way as to ensure that the goal of the research is accomplished. 


\section{MINORITY/SPECIAL POPULATIONS}

There are also a set of research issues relating to minority/special populations in which we need to invest more of our efforts and resources. First of all, I think we all know that drug abuse and addiction disproportionately afflict people in minority groups. Therefore, we have to pay far more attention to the issues surrounding these differences than we have in the past. In addition to this, there are an embarrassingly small number of minority researchers in our field. So we will need to be devoting significant amounts of energy to increasing the pool of minority researchers.

Historically, people have talked about recruiting minority researchers as an equity issue. I want to make it clear however, that for me, this is not an equity equity issue. This is not an issue about giving minority people chances. This is an issue about taking full advantage of the human resource pool available for science in this country. We have tremendously untapped resources and tremendously untapped expertise because we have not reached out in a very effective way to the community of potential researchers who come from minority groups. Thus, in the future, we will strive to be far more systematic about how we direct our efforts. This is not just an issue of fairness, it is a critical national need.

It is also the case that there are tremendous differences among subgroups within what are classified as minority groups. The two examples I know best are the "Asian" population and the "Latino" population. That is, the Asian population is of course made up of many different ethnic and cultural groups. And we have learned the hard way that by addressing all of the various factions collectively, we are not getting very far in finding workable solutions. The same is true for the Latino population. Here too, there are very real and significant differences; for example, between the Mexican American community and the Puerto Rican community. If we do not attend to these differences and continue our current practice of aggregating groups, we are not going to be successful in developing drug abuse prevention and treatment strategies that are effective with these groups. This is an area of NIDA's research we are actively attempting to strengthen.

A similar situation also exists in drug abuse research related to women. There is no question that the area of maternal/fetal drug effects is a very important one and one to which NIDA will have to devote sustained efforts. However, at the same time, it is clear that we have paid far too little attention to women, irrespective of their reproductive/maternal roles. We need to consider the fact that there are physiological, behavioral, and social gender related differences and we need to attend to the relevance of those issues per se. We will be expanding our activities in that realm as well.

\section{INFORMATION EXCHANGE}

One of the major messages that I have been conveying to people on the Hill and in the Administration is that NIDA wants to be useful. We want to be perceived as a part of this country's battle on drug abuse and addiction and we want the world to see us that way. This we hope to accomplish by moving our strategies involving information exchange into a more interactive mode than they have been in the past and by significantly broadening our community and constituency outreach and interaction efforts. And in order to ensure our research's relevance, we also need advice from people in the field about what questions we should be asking. 


\section{TOPICS IN THE MILL}

In addition to the areas already addressed, there are a number of other topics that we have begun discussing. I would like to briefly mention some of these activities that we have "in the mill".

\section{PROTECTIVE/RESILIENCE FACTORS}

Over the years we have spent a lot of time and a lot of effort attempting to identify factors which place certain individuals at risk for drug abuse and addiction. A vast number of such factors have been implicated, ranging from biological to psychological, social or environmental in nature. Since there appear to be so many risk factors for drug abuse which, in some cases, serve only to stigmatize those considered to be "at high risk", it makes sense to focus more efforts on identifying those characteristics which make certain individuals more resistant to drug abusing behaviors than others. Particularly where precursors of substance abuse are not amenable to change (such as economic deprivation), prevention programs can foster protective mechanisms that will increase the chances that participants can overcome their high-risk circumstances without turning to substance abuse. To gain that insight, in the future we intend to devote far more effort to the study of protective and resilience factors.

\section{EPIDEMIOLOGY RESEARCH}

In the area of epidemiology research, NIDA needs to move to the next generation. Here, we will focus efforts on improving existing methodologies for collecting and analyzing epidemiologic and ethnographic data and on increasing the reliability and validity of instruments that measure the nature and extent of drug abuse and dependence.

\section{PREVENTION RESEARCH}

Prevention research is also an area which we need to expand. Research has shown a reduction in drug use among young people who are exposed to comprehensive drug abuse prevention programs that promote the formation of anti-drug attitudes at all levels of society. We hope to do more in identifying effective comprehensive prevention interventions that use schools, media, family, peers, and community organizations to shape and reinforce positive self-regulated behavior changes. We will also support efforts to determine the efficacy of drug-free policies and legislation in a variety of settings. In addition to the more traditional approaches we have been taking to identify effective interventions, this is another area where we will be looking for novel approaches with demonstrated success in other fields to apply to the prevention of drug abuse.

\section{BEHAVIORAL SCIENCES}

Until recently, many areas of behavioral science that have relevance to drug abuse and addiction were not very prominent in our research portfolio. Because drug abuse and addiction is a disease expressed in behavioral ways, it is important that we concentrate more efforts on the study of the complex processes affected including learning and memory, motivation, and cognition. Clearly, this is an area in which I think we need to devote some strategic thinking. 


\section{CHILDREN AND ADOLESCENTS}

To a large extent, drug abuse and addiction are diseases of childhood and adolescence. The fact that they often have their origins there is an important point to consider in designing effective prevention and treatment strategies. Some of NIDA's public information activities have been fashioned with close attention paid to age differences. Since such targeted approaches offer the best hope of reaching their intended audience they will be employed more and more as new efforts are launched.

Clearly, the need to expand our knowledge about drug abuse and addiction has never been greater. The enormous health, social, and economic costs of using illicit substances; the apparent resurgence of illicit drugs among our youth; the devastating toll that drug abuse and addiction related HIV infection and AIDS continues to take on our nation's health all continue to heighten the urgency of the work that we do.

Fortunately, the opportunities for exploration and discovery in the drug abuse field have never been more abundant. And the technologies available allow us to venture into increasingly exciting areas. As NIDA begins its third decade, I look forward with great optimism to working with all of you in the drug abuse research community to surpass the remarkable achievements made thus far in the field, and to bring to those we seek to help, the best prevention and treatment strategies that science can offer.

AFFILIATION:

National Institute on Drug Abuse, Rockville, MD 20857 


\title{
INTRODUCTION TO THE NATHAN B. EDDY MEMORIAL AWARD RECIPIENT
}

\author{
J. V. Brady
}

It has been well over a quarter of a century since my path first crossed that of Jerome Jaffe, the honored recipient of this year's Nathan B. Eddy Memorial Award. It was the 1960s and we shared a role in that perennial agony we have come to know as "Study Section". Then, only in his early thirties, Jerry brought to the table an unparalleled reputation for scholarship as the author of the first "Drug Addiction and Drug Abuse" chapter in the prestigious Goodman and Gilman's, "The Pharmacological Basis of Therapeutics," a contribution he has continued to revise and update every five years for the past three decades. His accomplishments as an innovator of the model Illinois Drug Abuse Programs were equally well-known at the time and he was already credited with some of the earliest studies on long-acting pharmacotherapeutic agents for opioid dependence. His LAAM studies for example, had a 30-year jump on the FDA, about the lead time he continues to maintain over the rest of us in the drug abuse field.

Jerome Herbert Jaffe was born and bred in Philadelphia, bursting upon the scene as one of the early "New Deal" babies shortly after Independence Day, on July 6, 1933. His undergraduate and graduate degrees in Psychology, as well as his medical degree, were all awarded by Temple University between 1954 and 1958, and by 1959 he was a Resident in Psychiatry at the United States Public Health Service Hospital in Lexington, Kentucky. It was during this tour of duty in the Public Health Service that Jerry made early contact with the founding fathers in the field at the Addiction Research Center - Abe Wikler, Harris Isbell, Frank Fraser, among others - who were to have an abiding influence on his most productive career.

Within but a few years after returning to an academic appointment at the Albert Einstein College of Medicine in New York in the mid-1960s, he moved to the University of Chicago and the Directorship of the Illinois Drug Abuse Programs. And, at the ripe old age of 36, Jerry was summoned to service on the World Health Organization Expert Committee to rub elbows in Geneva with Dale Cameron and no less a luminary than Nathan B. Eddy, for whom this highest CPDD award has been named.

With this kind of a track record, it is little wonder that Jerry's accomplishments would come to the attention of the White House when the President found himself in need of a Special Consultant for Narcotics and Dangerous Drugs, and ultimately, a Director of the Special Action Office for Drug Abuse Prevention the first "Drug Czar". It was in those first few highly visible years in Washington during the early 1970s under Jerry's leadership that the first Federal Strategy was written and many of the programs that formed the basis for current research and treatment in drug abuse were initiated. Those years also witnessed the reversal of a 50-year policy prohibiting the use opioids in the treatment of narcotic dependence. As a result, methadone maintenance was approved and 
guidelines were developed for what has since proven to be the most effective available treatment for opioid dependence.

Jerry's return to teaching and research at Columbia and the University of Connecticut in the mid $70 \mathrm{~s}$ and early $80 \mathrm{~s}$ was characterized by yet another investigative initiative that proved to be a decade or two ahead of its time nicotine dependence. It was largely through his efforts that recognition of the disorder was acknowledged by inclusion in the DSM III diagnostic manual and he assumed a primary role as an expert witness in the first and only successful litigation against the tobacco industry.

Over the past decade, our distinguished Eddy Awardee has been back at the helm in the greater Washington megalopolis. He first served as Director of the relocated Addiction Research Center in Baltimore, then briefly as Acting Director of NIDA, and most recently at the PHS complex in Rockville, Maryland where he lent an expert hand to the establishment of the Office of Treatment Improvement with Beny Primm, and is presently on duty with the newly constituted Center for Substance Abuse Treatment.

At the ARC, Jerry was responsible for assembling the nucleus of the neuroscience group, now under the productive leadership of such luminaries as Mike Kuhar. In addition, he continues his own scholarly involvement through recently published reports on PET studies of drug effects in the human brain, adding to a pre-existing bibliography of some 300 or more research papers and authoritative reviews in the drug abuse field.

While this recognition of Jerome Jaffe's many accomplishments may be long overdue, it is nonetheless as richly deserved an Eddy Award as any this College has ever been honored to present for outstanding scientific and professional contributions to the field of drug dependence and abuse.

\section{AFFILIATION:}

The Johns Hopkins University School of Medicine

Baltimore, Maryland 


\title{
THE NATHAN B. EDDY LECTURE: SCIENCE, POLICY, HAPPENSTANCE
}

\author{
J. H. Jaffe
}

I am profoundly honored to have been selected to receive the Nathan B. Eddy Award. To be numbered among those previous awardees who have created and shaped this field is an unexpected event. I consider this recognition by my colleagues to be the high point of an academic/medical career that has been influenced (if not defined) by unexpected events. I would like to tell you about three of these events and the impact they had on my career and, indirectly perhaps, on drug abuse research.

In 1957, my third year of medical school, I read Abraham Wikler's book, "The Relation of Psychiatry to Pharmacology." This led me by a circuitous route to the Public Health Service Hospital at Lexington, Kentucky--not as a researcher, as I had hoped, but as a resident in psychiatry. Nevertheless, I attended the seminars of the great researchers of the Addiction Research Center: Harris Isbell, Abraham Wikler, H. Frank Fraser, William Martin, and others. I also developed lasting relationships with clinical researchers, such as Jack O’Donnell, James (Fred) Maddux, and Karst Besteman.

\section{EINSTEIN AND THE BLUE BIBLE}

I decided to seek more training in pharmacology before continuing my psychiatry residency, and was accepted as a post-doctoral fellow at Albert Einstein College of Medicine. I was attracted by the quality of the faculty in psychopharmacology. There was, too, a sense of excitement at the prospect of working in a department headed by Alfred Gilman, the co-author of "The Pharmacological Basis of Therapeutics"-- G \& G -- the "blue bible" I had used as a medical student just a few years before.

At Einstein, in 1961, Seth Sharpless and I hypothesized that the counteradaptation underlying physical dependence on centrally active drugs might be linked to the phenomenon of denervation or disuse supersensitivity. Our work on barbiturate physical dependence showed that, contrary to what was believed at that time, rebound hyperexcitability phenomena associated with barbiturate use developed very rapidly (Jaffe and Sharpless, 1965). The onset was perhaps as rapid as with opioids, which, as Wikler, Fraser and Isbell had shown in 1953, probably begins with the first dose. We also demonstrated rapidly developing tolerance and withdrawal-like phenomena after cholinergic blockade (Freedman et al., 1969).

In 1963, Murray Jarvik invited me to my first CPDD meeting. There I met Nathan Eddy and other luminaries in the field. Later that year, the first of the significant unexpected events of my career occurred. Lou Goodman and Al Gilman decided it was time for a 3rd edition of $G \& G$ and that the text would be multi-authored. To my great surprise, (since I had not ever published a refereed paper), I was asked to contribute two chapters, one on narcotic analgesics, and another--possibly the first in a general pharmacology text--on drug addiction. I was flattered to be asked to contribute and could not turn down such an invitation. 
A cardinal rule when writing for $G \& G$ was not to exceed the number of words or pages allotted. Every word counted, including citations and references; the more the citations, the less room for facts. Despite admonitions, my chapter on addiction exceeded the allowable length when I sent it to Lou Goodman in July of 1964. Goodman's exacting editorial style and encyclopedic knowledge were legendary - raw material for nightmares. I fully expected the chapter to be returned from his scrutiny with large blue marks on every page, and derisive comments of the kind he sometimes lavished on distinguished professors of pharmacology. Gilman had shown me one such manuscript with Goodman's comments, perhaps to help me focus my efforts. Instead, in Lou Goodman's characteristic bold hand, a brief and very complimentary comment appeared on the face sheet. He and Gilman decided to put the entire chapter into small print rather than cut it. Gilman expressed his view that, because the text was used throughout the world, my chapters might ultimately do more for patient care and for the field in general than anything that I could have accomplished at that time in the laboratory or in direct patient contact. (In retrospect, I'm not certain whether he was expressing confidence in the influence of $G \& G$ or some doubts about my clinical and laboratory skills.)

In the 29 years since the third edition of $G \& G$, the process of revising the chapters every five years has made it necessary for me to periodically revisit the entire literature in the field and has afforded me the wonderful opportunity to be a chronicler of the progress of knowledge on addiction and opioids. I welcome this occasion as an opportunity to express my appreciation to all of you who have contributed to what is now a vast body of scientific literature.

Because of my junior status in 1964, there was limited scope for my inherent iconoclasm. But I did put forward a conceptualization of drug dependence that differed from the authorities of the time. Eddy, Seevers, Halbach, and Isbell had not yet published their suggested revision of the WHO perspective that divided drug using behaviors into habits and addictions, the latter being distinguished by the presence of physical dependence. In an attempt to convey the complexity of the notion of compulsive drug use as I saw it, I wrote:

Addiction will be used to mean a behavioral pattern of compulsive drug use. ... Addiction is thus viewed as an extreme on a continuum of involvement with drug use and refers in a quantitative rather than a qualitative sense to the degree to which drug use pervades the total life activity of the user. Addiction in this frame of reference cannot be used interchangeably with physical dependence. (Jaffe, 1965a, p. 286.)

Some years later, the idea of a behavioral syndrome varying in severity and not equivalent to physical dependence was incorporated into the DSM-III-R generic definition of drug dependence. I found it curious even in 1964 that tobacco use was not generally viewed as an addiction, and I managed to squeeze this comment into an already over-length chapter:

Next to caffeine, nicotine (tobacco) is the substance most widely used for its effect on mood. ... The evidence that smoking is linked to cardiovascular and neoplastic disease and the inability of many persons to refrain from the use of tobacco in spite of efforts to do so suggest that this form of compulsive drug use will receive increasing attention in the future. (Jaffe, 1965a, p. 302) 
This was a cautious comment. After all, Al Gilman was a heavy smoker and there were limits to his faith in his junior staff. (Besides, nicotine was in the chapter on Ganglionic Stimulating and Blocking Agents.) In 1973, I included nicotine and tobacco in the first Federal Strategy for Drug Abuse and Drug Traffic Prevention; and, in 1974, I was permitted to include nicotine in the revision of the chapter on addiction for the 5th Edition (1975) of G \& G. I noted then that tobacco use, although rarely viewed as a form of drug abuse or an addiction, meets all of the accepted criteria for drug dependence. The following year, I had the satisfaction of working with Bob Spitzer to develop criteria for including tobacco dependence along with other forms of dependence in DSM-III; and in 1976 I persuaded William Pollin that NIDA should not continue to neglect nicotine.

At the time I wrote the chapter on narcotic analgesics, in 1964, physicians were so concerned about causing iatrogenic addiction that they were often unwilling to prescribe adequate doses of opioids for patients in pain. For that reason, I wrote:

Some degree of physical dependence and tolerance develop whenever a narcotic is given in therapeutic dosage over a prolonged period,... such considerations should not in any way prevent the physician from fulfilling his primary obligation to ease the patient's discomfort. ... no patient should ever wish for death because of his physician's reluctance to use adequate amounts of potent narcotics. (Jaffe, 1965b, p. 264)

As many of you have heard me say before, those last words, in italics, were for my father, who died of cancer--in pain. Despite my status on a pharmacology faculty, I had been unable to convince the attending physician that his fears of addicting my 70-year-old father were unfounded. Starting in 1975, I co-authored this chapter with William Martin, but this phrase was not changed.

\section{METHADONE, LAAM, AND CYCLAZOCINE}

By 1964, the problem of illicit drug use and heroin addiction had become a front page topic and arguments for heroin maintenance were being made by some respected scholars. Although my NIMH Career Development Award was based on laboratory work aimed at understanding physical dependence, I had become interested in exploring the clinical implications of opioid maintenance. I initiated a study aimed at testing the notion that opiate addicts become tolerant to the euphoric "rush" if allowed to use opioids i.v. over a prolonged period. I worked with a few patients maintained on hydromorphone i.v., which caused me to be visited regularly by an agent of the Bureau of Narcotics, but I was allowed to proceed. As I now recall, I learned of Vincent Dole and Marie Nyswander's work on oral methadone in early 1965, and quickly found in my own work that there was substantial benefit to both patients and therapists when oral methadone was the only opioid maintenance option.

At a CPDD meeting in February 1965, Martin, Gorodetzky and their coworkers suggested that cyclazocine, an opioid antagonist, might provide a means to test Abe Wikler's ideas about the role of conditioning. By September of 1965, I had designed a study, obtained supplies and, with my colleague, Leon Brill, launched a pilot study of cyclazocine. (Obviously, initiating clinical research was easier 30 years ago.) We presented our findings on 3-month's experience with cyclazocine at the CPDD meeting in February 1966, and our paper was published that year (Jaffe and Brill, 1966). The eagerness with which heroin addicts sought such treatment contradicted the belief that they were unmotivated to stop using drugs. It 
was obvious then that it would be useful to compare treatment with antagonists to treatment with methadone. I had gotten hooked on what works for whom.

Around this time I became interested in finding out if alpha-acetyl methadol (LAAM), a long-acting, methadone-like agent, would be effective in treating some of the addicts I was seeing. The slow onset and long duration of LAAM, described by Fraser and Isbell in 1952, seemed to be properties that could be advantageous for treating opioid dependence. Unable to locate any space on the Einstein campus for these clinical studies, I was happy to accept Daniel X. Freedman's offer to join his department at the University of Chicago and to carry out both basic and clinical studies of opioid dependence.

\section{THE UNIVERSITY OF CHICAGO AND THE ILLINOIS DRUG ABUSE PROGRAMS}

Although I formally joined the Chicago faculty in July of 1966, I did not move to Chicago until six months later. In the interim, I had the good fortune to work in Vincent Dole's laboratory at Rockefeller University. At the same time, I provided consultation to the newly established Narcotic Advisory Council of the State of Illinois--a role arranged by Danny Freedman.

In stark contrast to New York, Illinois had virtually no provisions for taking care of drug addicts. New York had a major detoxification hospital, a large-scale statesupported civil commitment program with active acquisition of residential facilities, and an expanding methadone program. There was also a growing therapeutic community movement sponsored by the City of New York which built on the techniques of Daytop Village and eventually gave rise to the Phoenix House program. The only way an addict could get help in Illinois was to plead guilty to a misdemeanor and then be detoxified under supervision in the infirmary of the jail -the Bridewell.

In January, 1967, I moved to Chicago and began to set up my laboratory at the University of Chicago. I also continued to provide consultation to the Illinois Narcotic Advisory Council. My advice can be quickly summarized. Addicts, I told them, are a heterogeneous group and we really don't know what treatment works best for which drug addicts. Therefore, Illinois should build a system that supports each type of treatment approach for which there is some evidence of efficacy; e.g., methadone maintenance, therapeutic communities, detoxification and post-detoxification aftercare with or without antagonists; then carefully evaluate the outcome for various patients, expand those methods that work, and shrink or eliminate those that don't. I also suggested that, despite the Congressional wisdom expressed in the Narcotic Addict Rehabilitation Act of 1966 establishing a Federal civil commitment program, civil commitment, already in the law in Illinois, should be held in abeyance. The Council decided that my advice made sense and said they would put through legislation to establish and fund a quasi-experimental treatment program for Illinois along the lines I suggested. However, they had one condition: I had to agree to run it. I knew from my New York experience that, for many people, treatment can make the difference between a decent life and one of stress and misery. I believed I had no real moral choice but to agree. This was the second unexpected event that sharply altered the course of my life.

Here ends the part of the narrative where the work involved just a few coworkers whose roles can be individually acknowledged. The next two episodes involve the work of many talented coworkers and facilitators who formed the teams for which 
I am only a spokesperson. In both instances, they are too numerous to list. Yet some played such vital roles that on this occasion their names must be mentioned.

Over the course of five years, Danny Freedman and the University of Chicago were generously supportive -- even more so than I realized at the time. So, too, were others in positions of authority, such as Governor Richard Ogilvie and Harold Visotsky, then Director of the Illinois Department of Mental Health, who responded with forbearance and resources to the unusual activities of what came to be called the Illinois Drug Abuse Programs (IDAP).

Within the first 18 months, IDAP had established a 15-bed detoxification ward at the hospital of the University of Chicago, residential and daycare facilities for patients who completed detoxification, several methadone maintenance programs, and a new therapeutic community--Gateway Houses Foundation, all in the immediate vicinity of the University. With Danny's help we recruited able scientists, like Bob Schuster and Patrick Hughes, to the University of Chicago and the Illinois Drug Abuse Programs. Others already in the Department of Psychiatry, such as Ed Senay and John Chappel, were seduced to get involved almost full time in the research and clinical work. Somewhat later, in mid-1969, we persuaded David Deitch and several of his top level coworkers at Daytop Village to come to Illinois - not just to strengthen the nascent therapeutic community that we had started at Gateway, but to help build firm links between the varied approaches we had initiated for helping opioid addicts recover and to develop a staff training program.

\section{PHARMACOLOGICAL AND EPIDEMIOLOGICAL STUDIES}

Although Dole and Nyswander had initially stabilized their patients on methadone on an in-patient basis, we found that our patients did quite well with totally ambulatory transfer from heroin to methadone. We also found that many patients showed remarkable improvement on relatively low doses of methadone. Our retention rates for these patients were comparable to those seen in New York. Further, about $20 \%$ of the first patients admitted to IDAP's methadone program elected to discontinue methadone but remained affiliated with the program. A few of these patients later required restabilization on methadone. The observation that some patients wanted to stop taking methadone and were able to do so successfully led us to the next study, which asked whether patients maintained at these lower doses were more likely to be able to successfully withdraw from methadone, when compared to patients maintained on doses closer to those that had been used in the studies by Dole and coworkers. In a study of patients randomly assigned to an average of 35 or $100 \mathrm{mg}$ of methadone we found, as others have subsequently, that the low dose group was more likely to have urine tests positive for illicit opioids. In this short study, the first of its kind, we did not find that lower doses were associated with successful withdrawal (Jaffe, 1970).

Early in 1969, Bob Schuster, Ed Senay, and I launched our first studies of LAAM and found that it had promise as an alternative to methadone (Jaffe et al., 1970). Patrick Hughes and his coworkers initiated work on the social structure of heroin using communities and on the epidemiology of heroin addiction (Hughes et al 1971-1972). John Chappel carried out work with cyclazocine and with mutual support groups (Chappel et al., 1971). 


\section{MULTIMODALITY AND ACCOUNTABILITY}

IDAP was founded with the intention of conducting a study of random assignment to maintenance, detoxification, and the Gateway therapeutic community. We actually initiated that study, but it proved impractical and it was discontinued after about eight months. Apart from the more formal scientific studies that were carried out and published, IDAP developed a system of treatment accountability that impressed the State of Illinois and assured us of continued support. We had been awarded one of six NIMH community grants in 1968, but by 1971 this provided only $14 \%$ of the program support. In the end we built a cohesive multimodality program in which the focus was on how to help people recover from addiction by finding which of the available programs worked best for them at a given time, rather than on which single treatment approach was better than another. It was a team approach that recognized the unique contributions of scientists, clinicians, and of former patients--many of whom developed skills and became program managers. The rancorous battles that characterized relationships among programs in New York seemed to have been avoided (Glasscote et al., 1972).

During the time we operated the inpatient detoxification unit, we noted that few major medical problems arose that required the resources of an acute medical unit. (The problem of HIV infection was not yet on the horizon.) But many problems did develop due to limitations on space and to hospital regulations and procedures designed to deal with different types of patients. In mid-1969, we closed the 15bed hospital detoxification unit at the University (much to the distress of Danny Freedman and the hospital administration) and used the same funding resources to open a new facility. The new program component was housed in an unused building, intended as staff housing, on the grounds of Tinley Park, a mental health center outside of Chicago. The building accommodated 100 people. Staffed only with a part-time psychiatrist, two nurses, and several counselors, the Tinley Park facility was nevertheless able to handle routine withdrawal. In addition, it provided a TC-like atmosphere for patients referred from other units of the program who, for a variety of reasons, needed more services or support than the existing ambulatory program could provide. Unlike the typical TC of the time, the duration of residence was adjusted to each patient's needs. Tinley Park served as a crisis center for outpatients on methadone, as well as for those who were not. All patients were expected to take responsibility within the community. Some patients stayed for a few weeks; others for several months; some brought their children with them. There was a separate program for adolescents. Tinley Park was also the training site for new staff and the presence of trainees enriched the program.

An even more complex multimodality unit, Safari House, was developed within IDAP in a building provided by the University of Chicago. Because it was located in the community, it served not only as a crisis intervention and residential unit, but also as an outpatient unit and day hospital. Like Tinley Park, Safari accepted women with children.

\section{THE SYSTEM AND THE PEOPLE}

As the program expanded and diversified, IDAP developed a central intake to address the gatekeeper function of conducting rapid assessments and helping to match patients with the treatment that seemed most appropriate. The objective was same day treatment. A centralized oversight responsibility made IDAP responsible for setting standards for staff qualifications, for treatment quality based on objective outcome measures, and for developing contracts between community 
organizations and IDAP for services. Payment for treatment services was based on capitation, an average cost for a year's treatment for a patient of average complexity. We also assumed responsibility for providing training and for the networking that would facilitate moving patients among treatment units as the needs of the patient changed. We put constraints on the use of acute hospital inpatient space and instead developed residential, day-hospital, and outpatient care. Two medical beds at a hospital on the north side of Chicago sufficed as medical backup for the entire program.

By late 1970, we also developed a "re-entry" clinic that allowed patients who had done poorly in one unit to get a fresh start, this time with more attention paid to concurrent psychiatric problems. When the waiting list got long, we started a "holding clinic," or pre-treatment methadone dispensary, attached to central intake to help actively addicted opioid users until a place in a regular treatment unit opened. In today's terminology, this would be called "interim" methadone. We knew it wasn't much, but it was the best we could do, and patients and staff believed it was better than nothing. At the same time, Patrick Hughes' community outreach team was showing us that people who don't seek treatment spontaneously may do so if only they are asked, and they do well if made to feel welcome.

Many interested people from the U.S. and abroad visited the Illinois Drug Abuse Programs to see what we were doing. One visitor who later played a major role in the field, and in several subsequent events, was Robert DuPont, who shortly thereafter initiated a major treatment program in Washington, D.C.

By July of 1971, IDAP had established more than 20 geographic units that were providing care to more than 2,000 people. The IDAP staff developed data on outcome of our methadone programs comparable to the studies done by other methadone programs. Gateway contracted for a follow-up of its residents. These data showed that a substantial percentage of patients had made major improvements in their lives, as well as reduced their drug use.

There are many kinds of evidence in addition to the follow-ups, urine tests, employment pay slips and rap sheets that researchers generally use as measures of improvement. At IDAP we also had the evidence of our day to day interactions with our patients. These interactions were not limited to brief office visits. We got to know dozens, if not hundreds, of patients quite personally. There were program picnics, baseball games, and celebrations. We met wives and husbands, reunited; children no longer ashamed of parents or worried that a parent might die or go to jail; parents no longer ashamed of their children. Patients and staff shared food and the pride of an enterprise jointly undertaken. Some of the celebrations were held in my home. My children were regular visitors at Illinois' first therapeutic community, in a large house two blocks from our own on Chicago's South Side. Former IDAP professionals only recently have told me that the great 1970 Fourth of July picnic at Tinley Park, when the staff and the patients of all the programs and their families joined together, remains one of the most memorable and emotionally uplifting events of their lives.

Here, listed alphabetically, are the names of some of the people at IDAP without whom none of this could have happened. Ed Senay and I agree that this is only a partial list: John Acheson; Ellen Afterman; John Chappel; Carl Charnett; Gail Crawford; Michael Darcy; David Deitch; William Duerk; Sam DiMenza; Barbara Evans; Nevin Fidler; Kirkland Fritz; Patrick Hughes; Krishan Kaistha; Herman Lancaster; Clarence Lawson; Mary Ann Manson; Pierre Renault; Charles R. 
Schuster; Edward Senay; Edward Washington; Matthew Wright; and Mischa Zaks. Some key IDAP staff included in this list began as patients. Their role in IDAP's success cannot be overstated.

\section{NATIONAL STRATEGIES AND THE WHITE HOUSE AD HOC COMMITTEE}

It was around the time of the Tinley Park picnic that some of the groundwork was laid that ultimately led to the third unexpected event. That summer, I spent several weeks in London with Griffith Edwards, of the Institute of Psychiatry, drafting a working paper for the WHO Expert Committee on Drug Dependence. The Vice-Chairman of the committee was Nathan Eddy. Over the few days of the meeting in Geneva, I came to appreciate why Dr. Eddy was held in such universal high regard. The working paper Griffith Edwards and I submitted to the Committee focused on questions of national strategies; e.g., how does a nation decide which varieties of drug use are of concern; who is charged with determining the responses to these concerns and specifying the goals; who is responsible for reviewing data to determine the degree to which the responses have been effective.

Shortly thereafter, I was contacted by Jeffrey Donfeld, a member of the Domestic Council staff at the White House. We had met previously when, Donfeld, at Bob DuPont's suggestion, visited IDAP in the course of looking at drug treatment programs throughout the U.S. Donfeld had become convinced, probably by DuPont's efforts in D.C., that much could be done about drug abuse just by expanding treatment. On behalf of the Domestic Council, he asked me to convene an ad-hoc committee of non-government experts to review and report confidentially to the White House on the Federal government's activities in drug abuse treatment, prevention, and research; and also to make recommendations on how to spend additional resources if they were made available. Over the eight weeks Donfeld allotted to the task, Edward Brecher, Sydney Cohen, Jonathan Cole, Gilbert Geis, John Kramer, William McGlothlin, Jack Mendelson, Helen Nowlis, John O'Donnell, Roger Smith and I carried out the review and drafted the report. Those of you who have attended these meetings for a long time will recognize that the group could have been a subcommittee of the CPDD.

The committee report recommended expanding treatment, including methadone; involving academic centers in the evaluation of treatment; and establishing an agency or office charged with developing and evaluating an overall national strategy. I heard nothing more from the White House until the following Spring, when I was invited to the White House to discuss a very different problem-heroin use in Vietnam.

\section{HEROIN, VIETNAM, SAODAP}

Eighteen years ago, in 1976, I described to a CPDD audience how my involvement in the Vietnam intervention was linked to President Nixon's announcement of the "War on Drugs" and the creation of the Special Action Office for Drug Abuse Prevention (Jaffe, 1976). That intervention was predicated on the belief that behavior could be altered more effectively by a modestly aversive but highly predictable consequence, such as a urine test and brief treatment in Vietnam, than by an improbably catastrophic consequence, such as a court martial. I also acknowledged my debt to a number of colleagues whose work provided the intellectual framework for that intervention. Among others, these included Abe Wikler, Bill Martin, Don Jasinski, and Avram Goldstein. 
What I did not describe in 1976 were the unexpected events surrounding the President's announcement on June 17, 1971. Based partly on the recommendations of the Ad Hoc Committee report, the White House had been preparing legislation to coordinate the Federal government's efforts to address the growing drug abuse problem. As I recall, on June 17th, I was invited to consult with the Domestic Council staff, and I fully expected to return to Chicago that evening. Instead, I was escorted to the Cabinet room and there, in front of a bipartisan group of Congressional leaders, President Nixon announced his plan for a War on Drugs and the establishment of a new office to run that war - the Special Action Office for Drug Abuse Prevention (SAODAP). He then introduced me as the person who would be in charge of the new office. This statement left me too shocked to say to the President that no one had asked me. It also left me wondering how to tell Faith, my wife, that we were moving again. She heard it for the first time on the news that night.**

I would like to mention briefly some of the work of SAODAP during its first two years. The challenges we faced were wide ranging. One goal was to make treatment so available that no drug users could say they committed crimes because they could not get treatment for their addiction. But which treatments, and on what infrastructure? NIDA did not yet exist. As far as the White House could determine, in all of the Federal agencies combined, only 250 people were involved with drug abuse research, education, grants administration and evaluation. This included those charged with implementing the NARA civil commitment programs.

At the same time, we needed to ensure a legislative base for the agency. Initially funded by Executive Order, without Congressional consensus on its necessity and authority, SAODAP would have run out of funding in less than a year. Then, a task of particular interest to me, there was the need to create a science base. And a special challenge to me, as a neophyte in government, was the explicit charge to coordinate the activities relating to drug abuse carried out by all the different departments and agencies of government. Once the legislation was enacted, we were also charged with developing a written strategy. If such a strategy was to be more rational in the future, we also had to develop the data necessary to determine whether the elements of the strategy were having the intended impact. These broad task descriptions encompass most of what we did in two incredibly busy years. But under each of these headings there were sub-tasks, some of which were themselves major challenges.

\section{DEMAND AND SUPPLY}

The Vietnam intervention continued to be a front burner issue. The results of the urine testing seemed to indicate that the intervention was having the intended effect: with each passing month, fewer Army personnel tested positive for opiates at time of departure. I used these data repeatedly to point out that sometimes less is more: that while all the MPs and electronic fences and threats of court martial could not bring heroin use under control, it was being reduced by properly arranged contingencies, even though the severity of the penalty had been reduced. The argument I presented internally within the Executive branch was that more can be accomplished by demand reduction than by supply control. These arguments were temporarily effective, as can be seen from budget allocations of that time. For a brief interval, policy was driven by data and results, and SAODAP was showing that at least one of its programs (the intervention in the military) was effective. 
A central theme was the expansion of treatment capacity. In doing so we used several principles developed at IDAP. We funded a variety of programs, including residential programs, but we limited the use of acute hospital beds. While residential and outpatient capacity grew, the percentage of Federally supported treatment capacity that used inpatient beds, including V.A. Hospitalbased programs, shrank from $6 \%$ to $4 \%$.

It was our intention to increase not just the number of programs, but their actual capacity and their geographic distribution. Just specifying treatment capacity was a problem, since up to that time grantees were not necessarily funded to treat any particular number of people and, once funded, they sometimes felt no particular concern if no one sought the kind of treatment they offered. We adopted the IDAP management concepts, including words like "slots," "static capacity," and "dynamic capacity," which probably have outlived their utility and await some equally simple concepts to replace them.

\section{METHADONE TREATMENT EXPANSION AND OTHER SMALL TASKS}

Legitimizing methadone maintenance and developing acceptable regulations for it was highly visible, highly controversial, and very expensive in terms of political capital. But it was only incidental to the overall mission of making effective treatment more than an afterthought in the national response to the drug problem. Within the first 18 months, the number of communities with Federally supported drug treatment programs rose from 54 to 214 and the number of programs to almost 400. We had developed more federally supported treatment capacity within the first two years than had been developed over the previous 50 years.

Paul Perito, the Deputy Director of SAODAP, and Grasty Crews (in the office of the general counsel) played critical roles in the process of establishing the legislative base for the office. The requirement for a formal written National Strategy emerged in response to a question at one of those numerous Congressional hearings associated with the SAODAP legislation, as various congressmen probed to learn how we would use the power of the office to solve problems. (Perito estimates that we testified more than 100 times before 11 committees in the House and the Senate over two years.) Other ideas that were converted into legislative authorities or mandates included special authorities to develop new pharmacological agents, and a national training center. Finally I learned to say less in front of Congress. The authority to establish a National Institute on Drug Abuse (NIDA) was written into the same legislation that created the Special Action Office, and it was my able successor at SAODAP, Bob DuPont, who initiated and was the first director of NIDA.

Here is a short list of some of the other projects that kept us busy: Vietnam intervention and follow-up; methadone treatment and methadone regulations; confidentiality regulations; Federal Strategy; NIDA research centers; naltrexone; LAAM; TASC; Career Teachers; National Training Center; Young Men \& Drugs; DAWN; CODAP; formula grants; management concepts and language; treatment in the V.A.; laboratory standards for urine testing facilities. I should at least comment at this meeting on the development of pharmacological treatments. The Veterans Administration study of LAAM that SAODAP designed and funded formed one of the pivotal studies that allowed NIDA, 20 years later, to obtain FDA approval. Clinical studies of naltrexone were also funded, and CPDD played a critical role in one of these studies. 
Last year's Eddy Award winner, Lee Robins, related how I contracted with her to conduct the Vietnam follow-up study and how happy the Department of Defense was to learn that relapse to heroin addiction was unexpectedly low (Robins, 1994). She did not tell you how I selected her to do the work, or how unhappy the DOD was initially with the idea of a follow-up study. I would like to acknowledge some other contributors to this classic study. Shortly after I was appointed "drug czar," I went to Vietnam (accompanied by my friend and colleague Beny Prirnm, among others) to inspect the intervention program I had designed and DOD had implemented. On my return, I reported to President Nixon at San Clemente. After hearing my report, the President said, "You should write a book about this; some of the greatest advances in medicine have come about from the lessons learned in wars." I took this as a directive, and so was born both the decision to conduct a follow-up study and the authority to see that it got done. Danny Freedman recommended Lee Robins as the best person to carry out the follow-up, and David Nurco, as a consultant to SAODAP, worked with Lee and reported back on any agency roadblocks to the study, all of which were grudgingly removed after phone calls from the White House to the reluctant agencies.

\section{SAODAP AND RESOURCE ALLOCATION}

It was obvious to me that more resources were needed for research on drug abuse. For those in this audience who have been in this field for less than 20 years, it is useful to be reminded how little research support there was in 1971 . In 1969 , in the last Johnson administration budget for drug abuse issues, the total for all Federal demand side activities and law enforcement efforts was less than \$100 million, with about \$50 million devoted to treatment and prevention. Although the treatment and prevention budget increased in 1970, in the initial FY 1971 budget, the total for research - clinical, basic, epidemiological, evaluation for all agencies was about $\$ 12$ million. The 1971 Supplementary amendment doubled the research budget. The growth in Federal funding for research, treatment, and training took place over a relatively few years and then was again flat. For a brief period, from 1972 through 1974, the balance between support for supply control and demand reduction activities shifted in the direction of demand reduction. In 1974, two-thirds of $\$ 750$ million of Federal resources for drug abuse went to treatment, research, education, and prevention. According to an analysis by the Institute of Medicine (Gerstein and Harwood, 1990). when adjustment is made for inflation, the absolute level of support for treatment and prevention was lower in 1989 than it was in 1974 . While the 1974 proportion of the resources allocated to demand side activities has not survived over the years, SAODAP's early decisions provided a solid beginning for research and a foundation for later expansion.

As the years have passed and my experience with government grows, I have come to appreciate what an extraordinary period that was, and what a supremely competent and dedicated group of people were assembled to work at SAODAP. Here is a partial list, in alphabetical order: Robert Angarola, Virginia Bannister, Deborah Hastings Black, Richard Boucher, Grasty Crews, Gerald DeAngelis, Lee Dogoloff, Jeffrey Donfeld, William Duerk, Alan Green, Jim Gregg, Ralph Howard, Thomas Keegan, Richard Klass, John Kramer, Brian LeBert-Francis, Mary Ann Manson, Bernard McColgan, John Meagher, Raymond Milkman, Stuart Nightingale, Vincent Nowlis, Paul Perito, Robert Pinko, Jack Richard, Kathleen Ross, A. John Rush, and Charles Yarbrough. 
I have already mentioned the vital role Paul Perito played as Deputy Director. In addition, to the small staff of SAODAP itself, we were able to use consultants who could not make a full time commitment. Among the consultants who gave generously of their time and expertise were John Ball, Roger Meyer, Jack Mendelson, David Nurco, and Beny Primm.

With the exception of the data on the efficacy of methadone maintenance, the great expansion of treatment in 1971 was based largely on faith and persuasive advocacy. In the intervening 23 years, we have developed not only better methods for assessing the efficacy of treatment, but better questions as well. We know a lot about the characteristics of patients likely to do poorly. We also know a lot about what makes a good program - but improving poor programs involves management and resources issues more than science.

\section{TREATMENT, SCIENCE AND RESOURCE ALLOCATION}

It seems to me that with respect to the science of the treatment of drug dependence several knotty problems remain - and they are probably interrelated. First there remains the puzzling and sometimes inspiring capacity of drug dependent individuals to improve as a result of a variety of life changes, not all of which can be thought of as treatment. For example, Lee Robins told us last year about opioid dependent drug users who regained control over drug use unrelated to any identifiable intervention. A follow-up of some addicts from the early years of the California Civil Commitment program found that those who had achieved abstinence from opioids (albeit only 30\%) generally had positive views of their treatment experience under the program, but frequently attributed their recovery to religion as well (Bailey et al., 1994). George Vaillant (1973) long ago told us of the relationship between parole supervision and recovery from opioid dependence. There are also those who achieve recovery as a result of treatment in therapeutic communities. Even when we look at treatment with methadone we find that, while many patients do best when maintained on methadone indefinitely, there are those 15 to $30 \%$ who find a way to do well after withdrawal from methadone.

Despite our efforts thus far, we are still not able to predict with great accuracy which individuals will respond best to which treatment at a particular stage in their drug-using careers. While we may be making some progress in matching alcoholics to specific treatments, in the case of cocaine dependence we are probably no further along than we are with opioid dependence. For the work-aday clinician there is still a need for data to help with the decisions about what to recommend to that next patient who comes through the door.

Perhaps related to our current inability to specify the one best treatment that will help a given individual at a given time, there persists in some influential quarters a disbelief in the efficacy of treatment in general. Too often I have heard individuals, who until recently were in policy making positions, say that treatment just doesn't work. Without the efficacy of supply control interventions being questioned, for the past 18 years Federal resources were disproportionately directed at crop control, border interdiction, and law enforcement activities aimed at control of local drug distribution. A recent RAND analysis suggests that available data indicate that treatment is far more cost effective in reducing cocaine use than are crop control, border interdiction, or even domestic law enforcement (Rydell and Everingham, 1994) a point I believed but could not prove 23 years ago. 
Without waiting for Congress to act on health care reform, private insurers have been carving out treatment of drug problems as an area where sharp cost reductions are possible. Unfortunately, even as the data mount showing that time spent in treatment is a powerful predictor of outcome for many patients, the amount and duration of treatment is being reduced along with costs. To an undetermined extent, the trend to move medicaid patients into managed care arrangements is probably reducing the time spent in treatment. The system seems to be responding to factors other than data. It would seem that some fiscal decision makers have drawn the inference that since so many interventions work for some people, then all interventions are probably equally efficacious, and we might as well emphasize those that cost the least and offend the sensibilities of the fewest. Take heart. Press on with clinical and basic research. Every once in a while an unexpected event occurs and data competently developed and honestly interpreted regain their capacity to influence policy.

On behalf of my wife, who has been responsible for almost every manuscript I have ever turned in; my children, now grown, who six times sacrificed their dining room table at the altar of the blue bible (and once for the first Federal Strategy); and on behalf of my coworkers at IDAP and SAODAP and those in the Federal bureaucracy and Congress who made SAODAP make a difference, I gratefully accept this Nathan B. Eddy Award.

\section{** ADDENDUM}

Shortly after I gave this lecture, I found a memorandum written by Jeffrey Donfeld describing a meeting in the Oval Office on June 10, 1971, at which, apparently, I first met President Nixon and discussed the plan for the Vietnam intervention. The memo also describes my suggestion that it was important to do a follow-up study of that intervention. The discovery of the Donfeld memo led Faith to search out the press clippings that she and other family members had saved. During the days following the Oval Office meeting, my name and picture appeared in the media describing me as the person likely to head a major program dealing with drugs. It is clear that I must have known that I might be asked to play some role in a new program. Nevertheless, the emotional impact of being publicly named by the President to head the program at the meeting in the Cabinet Room on the 17th --without my having been formally offered such a position -was such that, for the 23 years since then, I have mis-remembered the precise chronology of the events.

\section{REFERENCES}

Bailey, R.C.; Hser, Y.I.; Hsieh S.-C.; and Anglin, M.D. Influences affecting maintenance and cessation of narcotics addiction. I Drug Issues 24:249-292, 1994.

Chappel J.N.; Senay E.C.; and Jaffe J.H. Cyclazocine in a multimodality treatment program: comparative results. Int $\underline{\mathrm{J}} \underline{\text { Addiction }}$ 6:509-523, 1971.

Eddy N.; Halbach H.; Isbell H.; and Seevers M. Drug dependence, its significance and characteristics. Bull Wrld Hlth Org 32:721-733, 1965.

Freedman, M.J.; Jaffe, J.H.; and Sharpless, S.K. Central nervous system supersensitivity to pilocarpine after withdrawal of chronically administered scopolamine. J Pharmacol Exp Ther 167:45-55, 1969. 
Gerstein, D.R. and Harwood, H.J., eds. Treating Drug Problems, Washington, D.C.: National Academy Press, 1990.

Glasscote, R.M.; Sussex, J.N.; Jaffe, J.H.; Ball, J.; and Brill, L. The Treatment of Drug Abuse: Programs, Problems, Prospects, Washington, D.C.: American Psychiatric Association, Joint Information Service, 1972.

Hughes, P.H.; Barker, N.W.; Crawford, G.A.; and Jaffe, J.H. The natural history of a heroin epidemic. Amer J Public Health (July) 1972.

Hughes, P.H.; Crawford, G.A.; Barker, N.W.; Schumann, S.; and Jaffe, J.H.

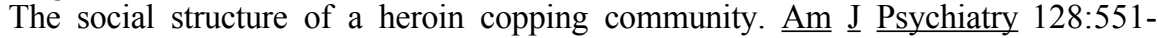
$558,1971$.

Jaffe, J.H. Drug addiction and drug abuse. In: Goodman, L. and Gilman, A., eds. The Pharmacological Basis of Therapeutics. New York: The Macmillan Co., 1965a, pp. 285-311.

Jaffe, J.H. Narcotic analgesics. In: Goodman, L. and Gilman, A., eds. The Pharmacological Basis of Therapeutics. New York: The Macmillan Co., 1965b, pp. 247-284.

Jaffe, J.H. Further experience with methadone in the treatment of narcotics users. Int $\underline{\mathrm{J}}$ Addiction 5:375-389, 1970.

Jaffe, J.H. Looking back around the corner. In: Proceedings of the 38th Annual Scientific Meeting. Committee on Problems of Drug Dependence, National Academy of Sciences - National Research Council, Washington, D.C., 1976, pp. 429-440.

Jaffe, J.H. Footnotes in the evolution of the American national response: some little known aspects of the first American Strategy for Drug Abuse and Drug Traffic Prevention. Brit Addiction 82:587-600, 1987.

Jaffe, J.H. and Brill, L. Cyclazocine, a long acting narcotic antagonist: its voluntary acceptance as a treatment modality by ambulatory narcotics users. Int $\underline{J}$ Addiction 1:99-123, 1966.

Jaffe, J.H.; Schuster, C.R.; Smith, B.B.; Blachly, P.H. A comparison of acetylmethadol and methadone in the treatment of chronic heroin users. A pilot study. JAMA 211:1834-1836, 1970.

Jaffe, J.H. and Sharpless, S.K. The rapid development of physical dependence on barbiturates. J Pharmacol Exp Ther 150:140-145, 1965.

Robins, L.N. The Nathan B. Eddy Lecture: Challenging Conventional Wisdom About Drug Abuse. In: Harris, L.S., ed. Problems of Drugs Dependence, 1993; Proceedings of the 55th Annual Scientific Meeting. The College on Problems of Drug Dependence. Inc., NIDA Res Monogr 140:30-45, 1994.

Rydell, C.P. and Everingham, S.S. Controlling Cocaine. Supply Versus Demand Programs, Santa Monica: RAND, 1994.

Special Action Office for Drug Abuse Prevention. Annual Report, 1973, Washington, D.C., U.S. Govt. Printing Office, 1973. 
Strategy Council on Drug Abuse. Federal Strategy for Drug Abuse and Drug Traffic Prevention 1973. Washington, DC.: The Strategy Council, 1973.

Vaillant, G.E. A 20-year follow-up of New York narcotic addicts. Arch Gen Psychiatry 29:237-241, 1973.

Wikler, A. The Relation of Psychiatry to Pharmacology. Baltimore: Williams \& Wilkins, 1957.

\section{AFFILIATION}

Center for Substance Abuse Treatment/SAMHSA

Rockwall II - 8th floor, 5515 Security Lane

Rockville, MD 20852 


\title{
THE RELATIONSHIP OF SCIENCE TO DRUG ABUSE POLICY
}

\author{
H. Kleber, R. Weich, A. Solarz, and M. Kleiman
}

According to many, drug abuse is the number one ethical, moral, legal, social, and public health problem that faces the United States today. Further, since different members of our pluralistic society conceptualize this multi-faceted problem in different ways, the social and public health policies suggested for its solution are diverse and often contradictory. Finally, as is the case with most contentious areas of human behavior, there is often an inverse relationship between the passion with which people express their views about a policy and the availability of evidence supporting its effectiveness. Consequently, it is with some fear and trepidation that a scientist enters this arena.

Nonetheless, in this symposia the participants have attempted to bring some light to the discussion of the potential role of science in the conceptualization, elaboration, implementation, and evaluation of policies in the area of drug abuse. It began with comments by the chairs on the way in which policies are often predetermined by the way problems are conceptualized, and then moved to the participants who discussed some specific ways in which science and drug abuse policy interact, and the obstacles which must be overcome to facilitate this interaction.

To start with, it is important to identify who the policy makers are and how they can best be informed about relevant science. Dr. Herb Kleber provided his unique perspective as the former Assistant Director of the Office of National Drug Control Policy, and Mr. Ron Weich from his perspective as a Congressional staff member dealing with drug abuse policy and legislation for Senator Edward Kennedy. Dr. Andrea Solarz discussed her experiences as a science advocate in attempting to educate congressional staff on the latest biobehavioral research findings that are relevant to drug abuse, and Dr. Mark Kleiman served as discussant.

In his presentation, Mr. Weich gave the conference attendees an appreciation of the rapid pace and time sensitivity of policy making, speaking via telephone from his office in Washington, D.C. during a break in ongoing staff meetings, on the legislation which would later become the Omnibus Crime Bill of 1994. He emphasized (and demonstrated) that the pace of legislative activity on drug-related issues is sometimes quite rapid and often enmeshed within much larger legislative initiatives. The bill he was working on at the time was an excellent example, given that millions of dollars of drug abuse treatment provisions (although not research) were included among the many anti-crime provisions of the bill. He acknowledged the important work of the College and of the research community; noting that although data may often be used inappropriately to lend support to a certain partisan position, in the final analysis the knowledge gained from the body of research on drug abuse and dependence is extremely valuable in guiding the legislative process. 
Herb Kleber was the Deputy Director of Demand Reduction in the Office of National Drug Control Policy for two years, where he had responsibility for setting the national strategy for reducing the demand for illegal drugs. He also has a long and distinguished career in research in the substance abuse field, and brought this wealth of first hand experience in both the policymaking and scientific realm to the symposium. He noted that policy should ideally be driven by timely and accurate scientific data. Unfortunately, often either such data does not exist or exists in a form not usable or useful for policymakers. There are, of course, those not infrequent episodes where good data does exist and is ignored, for example, the Swedish Methadone Study.

There are many policy issues that could benefit from accurate data, and Dr. Kleber reviewed several of them. For example, many call for alternatives to incarceration or for increased treatment in prison. However, Attorney General Janet Reno has emphasized that prison cells are a scarce commodity in many communities and should be used to their maximum advantage. This speaks to the need for more information on predicting dangerousness and recidivism as well as exploring possibilities for shorter but surer and swifter prison sentences.

Funding for treatment in the recent past has been a bipartisan failure -- the Republican administration, under President Bush, asked for insufficient funds and the Democratic controlled Congress provided about 1/3 of what increase was requested. This stems from a lack of belief in treatment efficacy, both among policy makers and the general public. No one ever lost an election by not voting for more funds for treatment, but many have won or lost depending on whether they called for more police. This, of course, also relates to the moral belief that the public should not be paying for treatment for individuals who brought their problems on themselves, although we ignore that for other diseases, such as heart attacks related to life style issues.

Dr. Kleber summarized the primary research needs for drug policy in three areas: epidemiology, treatment, and prevention. In the area of epidemiology, he identified the critical need for better data on the numbers and characteristics of addicts: how many individuals are addicted to various drugs?; what are their relevant demographic and psychological characteristics?; how many need treatment?; and what kind(s) of treatment are needed? Also, critical to development of policy is valid, reliable and timely data on changes in drug use in various segments of the population. In the treatment area, he identified several important needs. First, there is a lack of data on what happens to individuals once they enter the treatment system, the "natural history" of treatment services. Second, there is a need for better information on what treatment services work best for what individuals, that is, on matching patients to the optimal treatment modality and services. Finally, we need to do a better job of measuring treatment outcome in ways that make sense and are useful to the policymaker. Finally, in the area of prevention, we need to collect more information on factors which are protective against drug abuse and addiction; and how to target our prevention efforts to different segments of the adolescent population.

Dr. Kleber identified several policy areas that he thought were ready for change. He called for a more equitable division of anti-drug funding between supply reduction and demand reduction activities, which would include expansion of treatment services capacity to 2.5 million episodes per year. He also called for increased emphasis on addicts involved with the criminal justice system; for example, by utilizing therapeutic communities as alternatives to prison; and for 
increased efforts to improve the comprehensiveness of treatment to include HIV and TB services.

Andrea Solarz, Assistant Director for Science Policy of the American Psychological Association, titled her presentation, "Scientists and Policymakers: Strange Bedfellows?" In it she noted that there actually is a relationship, albeit tenuous, between the two. Unquestionably, national policies do get developed without being adequately informed by what we have learned from relevant research. At the same time, however, there are many opportunities to get the results of scientific study into the policymaking process. The major challenges facing organizations that are trying to foster a greater appreciation for the contributions of science to the policy process, are informing Congress about the important contributions behavioral science can make to the policymaking process, and convincing scientists that they have an important role to play in this activity.

Few scientists are actively involved in public policymaking, and many actually resist involvement. There arc a number of reasons for this: they are uncomfortable with the policy world and the policy process, which seems unmethodical and unpredictable; they may not believe that advocacy is an appropriate role for scientists; or they may feel that they arc not well enough informed or skilled to participate. There are many reasons why policymaking should be important to scientists. For example, there arc a number of direct "pocketbook" issues. Congress decides how much money will go to the federal research institutes, and often directs what areas should be funded. Congress may also direct what types of studies CANNOT be done, sometimes targeting studies that had previously been approved through peer review. Legislation may even direct HOW research can be conducted, for example, by regulating the use of animals in research or restricting the types of questions that can he asked of human subjects.

Scientists have significant information to contribute. There are many areas where behavioral scientists offer special insight and expertise because of their knowledge of behavior, for example, in the areas of anti-crime legislation, welfare reform, or drug prevention legislation. Finally, the involvement of scientists is critical because if they don't participate, there are many other voices competing for attention, and those are the ones that will be heard. There is not presently a loud advocacy voice on Capitol Hill with a primary focus on substance abuse research. Most of the organizations in Washington that focus on substance abuse are interested primarily in services issues. And although most would agree that research is important, their support is incidental to the main work that they do.

Scientists should become more involved and can, through professional associations, such as the American Psychological Association. For example, the APA often invites members to present testimony before Congress, brief Congressional staff on research relevant to critical policy issues, and help identify gaps in the field. While few scientists pursue interests in policymaking by actually working on Capitol Hill, there are many other ways in which they can be involved. Visits to legislators, or calls or letters on legislation can have real impact. And, scientists can help to educate policymakers about the importance of science and its contributions to the development of sound public policy, for example by actually hosting a visit to their institution.

The relationship between policymakers and scientists is one that works both ways: what policymakers do affects science; hut what scientists do can influence policymakers as well. Clearly, a choice does not need to be made between doing 
science and doing public policymaking. Scientists have much to contribute to the process, and can do so even without a big commitment of time or an in-depth understanding of the process.

The discussant, Mark A. Kleiman, noted that the influence of science on policy may be less powerful than the influence of policy on science. In the desired arrangement, key uncertainties whose resolution would inform important policy choices are identified, and research resources are directed toward activities that might resolve those uncertainties. More common, however, are situations in which research resources are made differentially available for projects which fit pre-conceived notions or current drug policy. In today's political environment, that means research which reinforces the public fear of drugs (to reduce prevalence) and of drug users (to create political support for prevention, treatment, and enforcement). The situation is further complicated by the legislative distinction between licit and illicit drugs. Obviously, from a scientific standpoint, the current legal status of drugs should not but too often does affect research. Several examples of how data can be used to support one policy position over another were given to illustrate the complex and evolving relationships between science and drug policy.

\section{Conclusion}

Policies are determined by many different factors in addition to scientific data. Political and economic considerations, as well as ethical and moral judgments enter into the decision-making process of policymakers. Which of these influences predominates depends not only upon the issue but as well, the "zeitgeist" of the society. The principle role of the scientist is to provide, where possible, scientific data to assist in the policy-making process, recognizing that only rarely will such contributions be determinative of the outcome. It is hoped that symposia, such as this, will provide a context in which scientists and policymakers and policy analysts can begin a discourse that will lead to greater communication and understanding. In this manner the CPDD membership may become of greater assistance to policy makers in the establishment and evaluation of drug abuse policy. In fact, several mechanisms already exist, through the auspices of the American Psychological Association and the American Association for the Advancement of Science, for researchers to contribute directly to the legislative policy process through fellowship programs. Attendees were encouraged to become more actively involved in science policy-making through these and other programs.

\section{SUMMARY}

Steven W. Gust

\section{AFFILIATIONS}

Columbia University, New York, New York

U.S. Senate Labor and Human Resources Committee, Washington, D.C.

John F. Kennedy School of Government, Washington, D.C.

American Psychological Association, Washington, D.C.

National Institute on Drug Abuse, Baltimore, MD 


\title{
S Y M P O S I U M F O R 2 OT T H A N N I V E R S A R Y CELEBRATION OF NIDA: UPDATE ON OPIOID RECEPTOR CLONING: IMPLICATIONS FOR RESEARCH ON ADDICTION
}

\author{
M. J. Kreek; E. Simon; C. Evans; G. Uhl; J. B. Wang; P. \\ Johnson; Y. Imai; D. Walther; J. M. Wu; W. F. Wang; A. \\ Moriwaki; L. Yu; B. Min; L. Augustin; R. Felsheim; J. Fuchs; H. \\ Loh; and C. Inturrisi
}

\section{Characterization of Opioid Receptors at the Molecular Level \\ Chris Evans, Ph.D., Department of Psychiatry \& Biobehavioral Sciences, University of California, Los Angeles, CA}

The molecular characterization of the opioid receptors at the nucleic acid level has begun to reveal the features that constitute the heterogeneity that has been observed for two decades in both opioid binding sites and the physiological action of different opioids. The original isolation of ad opioid receptor cDNA from the neuroblastoma/glioma cell line NG108-15 provided the template from which the $\mu$ and $\kappa$ opioid receptors have subsequently been identified. These three cloned receptors, which thus far constitute the opioid receptor family, have high interhomology $(70-80 \%$ identity) with the exception of the $\mathrm{N}$ - and $\mathrm{C}$ terminal domains and the third and fourth extracellular domains. The homology profile is highly consistent with the observation that all three receptors act through similar intracellular signalling systems although with somewhat different ligand selectivities. The repeated isolation of identical $\mu, \partial$ and $\kappa$ receptor sequences has raised questions as to the etiology of the subtypes of these receptor classes i.e., whether subtypes such as $\partial 1 / \partial 2$ indeed have different primary structures. The lack of appropriate clones for these subtypes has reinstated the hypothesis that the environment of the receptor or post-translational changes may contribute to the observed pharmacological heterogeneity.

Genomic analysis has identified multiple introns in the protein coding region of all three opioid receptor genes and conserved features in the intron/exon boundaries. Unlike some G-protein coupled receptor families, the human opioid receptor genes are not clustered and have been mapped to distinct chromosomes with the delta on chromosome 1 , the kappa on chromosome 8 , and the mu on chromosome 6 .

In situ hybridization with cRNA probes in both human and rodent tissues has begun to reveal the individual cells expressing the $\partial, \mu$, and $\kappa$ opioid receptors. The distribution of mRNA encoding the receptors is, in the main, consistent with prior mapping studies based on radioligand binding and autoradiography. For example, the distinct patch distribution of mu receptor binding in rodent striatum is consistent with a patch-like distribution of the $\mu$ receptor mRNA, whereas in human striatum both the $\mu$ receptor mRNA and binding does not appear to conform to an obvious patch/matrix distribution. In situ has revealed a number of additional areas in rodent brain that may express opioid receptors including the motor neurons of the ventral horn which express $\partial$ receptor mRNA and the deep cerebella nuclei which express both $\mu$ and $\partial$ receptor mRNA. Clearly the nucleic acid and antibody probes derived from the cDNA and genomic clones will considerably enhance our understanding of the neuroanatomy of the endogenous opioid receptors. 


\section{Human Opiate Receptors and Their Genes}

George Uhl ${ }^{\# !++}$, Jia-Bei Wang ${ }^{\#}$, Peter Johnson", Yasuo Imai ${ }^{\#,}$ Donna Walther, Jun Min Wü, Wen-Fei Wang", Akiyoshi Moriwaki", "Molecular Neurobiology Branch, *Office of the Director, Intramural Research Program/NIDA, 'Pharmacology Research Associate Program, NIGMS; ${ }^{+}$Depts. of Neurology and Neuroscience, Johns Hopkins University School of Medicine Baltimore, MD

$\mu$ Opiate receptor distributions and pharmacologic properties place them among the receptors most identified with the analgesic and addicting properties of opiate drugs. Recent studies have identified cDNAs encoding rodent and human $\mu$, and $\partial$ and rodent $\kappa$ opiate receptors. The availability of these cDNAs has allowed us

to define the structure of the human $\mu$ opiate receptor gene, to identify human $\boldsymbol{\kappa}$ opiate receptor cDNAs, to stud developmental and other patterns of the $\mu$ opiate receptor's regulated expression, and to identify structure-function relationships in these interesting members of the seven transmembrane domain, G-protein linked receptor family. Analysis of $\geq 50 \mathrm{~kb}$ of genomic sequence reveals that the human $\mu$ receptor gene contains three introns interrupting four protein-coding exons, ranging in size from 359 to $>750$ base pairs. Studies with chromosomal in situ hybridization and rodent/human hybrid cells both indicate localization of a singlecopy gene to chromosome 6, bands q24-25. Ribonuclease protection assays revealed the most abundant $\mu \mathrm{OR}$ mRNA was in thalamus, hypothalamus, midbrain, and spinal cord. In situ hybridization studies reveal that subpopulations of neurons in several thalamic nuclei express $\mu$ OR mRNA, with most abundant expression in neurons of the medial aspect of the lateral habenula. $\mu \mathrm{OR}$ immunoreactivity was detected in the dorsal horn of the spinal cord, nucleus caudalis of the trigeminal nuclear complex, the vagal nuclei, nucleus ambiguous, the locus coeruleus, the periaqueductal grey, and other brainstem regions. These distributions of mRNA and protein appear in developmental patterns that display regional specificity. A subcloned PCR amplification product from human brain cDNA which encoded amino acids corresponding to the putative second extracellular loop of the human $\mathrm{K}$ opiate receptor sequence was introduced into the human $\mu$ opiate receptor by recombinant PCR. The N/K chimera retained high binding affinities for morphine, DAMGO, and naloxone; while, its affinities for a-neoendorphin, dynorphin A (1-13), and dynorphin A (1-17) were increased by more than 250-fold, 100-fold, and 10-fold, respectively, when compared to affinities for these peptides displayed by the wildtype $\mu$ receptor. The chimeric receptor was fully active in cyclase inhibition mediated by morphine and dynorphin A. These experiments appear to identify substantial contributors to the "address" aspect of dynorphin recognition.

\section{Opioid Receptors: Molecular Cloning and Cellular Function}

Lei Yu, Ph.D., Department of Medical and Molecular Genetics, Indiana University School of Medicine, Purdue, IN

The $\mu$ opioid receptor represents the high affinity binding site for the opioid narcotics with high abuse liability such as morphine, codeine, methadone, and fentanyl. In addition, heroin (diacetylmorphine), a semi-synthetic derivative of morphine, crosses the blood-brain barrier much more readily than morphine due to its increased hydrophobicity. Once in the brain, heroin is rapidly hydrolyzed to morphine, which acts at the $\mu$ opioid receptor and results in an euphoric effect, thus conferring the reinforcing properties of the drug and contributing to the development of drug dependence. Because of its high affinity for these aped narcotics, the $\mu$ receptor is considered the main cellular mediator in the 
narcotics, the $\mu$ receptor is considered the main cellular mediator in the development of tolerance and opioid addiction (Di Chiara and North, 1192). Using a strategy of low stringency hybridization for isolating opioid receptors (Chen et al., 1993) related to the mouse $\partial$ opioid receptor, we have cloned the cDNAs for the $\mu$ opioid receptor from the rat and human brain. Upon transfection into cultured mammalian cells, the cDNA clones express the $\mu$ opioid receptor with $\mathrm{nM}$ affinity for opioid narcotics, as well as for endogenous opioid peptides ß-endorphin, Met- and Leu-enkephalins, and dynorphin A (1-17).

The $\mu$ opioid receptor is known to exert two types of inhibitory effects on a cell: reduction of the intracellular level of cAMP; and inhibition of neuronal firing. Using the cloned $\mu$ opioid receptor, we have started to study the molecular mechanisms for both of these processes. A mammalian cell line has been established in which the $\mu$ opioid receptor is expressed at a high level. Acute activation of the $\mu$ receptor results in a decrease in the intracellular cAMP. After the cells are chronically exposed to morphine, withdrawal of morphine causes a rebound in cAMP, suggesting that the cells may be used as a model for study of the biochemical changes that may occur in aped dependence. When expressed in amphibian oocytes, the $\mu$ opioid receptor inhibits a low voltage-activated $\mathrm{Ca}^{2+}$ channel and stimulates a $\mathrm{G}$ protein-activated $\mathrm{K}+$ channel. Upon repeated stimulation, the $\mu$ receptor-coupled $\mathrm{K}+$ current is desensitized. Interestingly, the desensitization is modulated by protein kinases: the cAMP-dependent protein kinase (PKA) blocks desensitization, whereas both the protein kinase $\mathrm{C}$ (PKC) and the type II $\mathrm{Ca}^{2+}$ /calmodulin-dependent protein kinase (CaM kinase II) potentiate desensitization. Our results suggest that these cellular models are useful in studying the cellular function and tolerance development involving the $\mu$ opioid receptor (Chen and $\mathrm{Yu}, 1994)$.

\section{References}

Chen, Y.; Mestek, A.; Liu, J.; Hurley, J.A.; and Yu, L. Molecular Pharmacology 44:8-12, 1993.

Chen, Y., and Yu, L. Journal of Biological Chemistry 269:7839-7842, 1994.

\section{Genomic Structure and Analysis of Promoter Sequence of a Mouse $\boldsymbol{\mu}$ Opioid Receptor Gene}

Bon H. Mink, Lance B. Augustin*, Roderick F. Felsheim*, James A. Fuchs", and Horace H. Lob*, * Department of Pharmacology, University of Minnesota, Minneapolis, MN; `Department of Biochemistry, University of Minnesota, St. Paul, MN; and \&Department of Biochemistry, Kon-Kuk University Choongjucity, Korea

We have isolated mouse $\mu$ opioid receptor (MOR) genomic clones containing the entire amino acid coding sequence corresponding to rat MOR-1 cDNA, including additional 5' flanking sequence. The mouse MOR gene is $.53 \mathrm{~kb}$ long, and the coding sequence is divided by three introns, with exon junctions in codons 95 and 213 and between codons 386-387. The first intron is .26kb, the second $.8 \mathrm{~kb}$, and the third $.12 \mathrm{~kb}$. Multiple transcription initiation sites were observed, with four major sites confirmed by 5'RACE and RNase protection located between 291 and 268 bp upstream of the translocation start codon. Comparison of the 5' flanking sequence with a transcription Factor database revealed putative cis-acting regulatory elements for transcription factors affected by cAMP, as well as those involved in the action of gluco- and mineralo-corticoids, cytokines and immune cell specific factors. 


\section{Mapping and Modulation of Opioid Receptor Gene Expression}

Mary Jeanne Kreek, M.D., Laboratory on the Biology of Addictive Diseases, The Rockefeller University, New York, NY

For several years our laboratory has had a major research interest in the possible role of the endogenous opioid system in three major addictive diseases: heroin addiction, cocaine dependency, and alcoholism. We have shown in basic clinical research studies that the endogenous opioid system components involved in hypothalamic-pituitary-adrenal axis as well as hypothalamic-pituitary-gonadal axis, important stress responsive and reproductive biological systems, are profoundly disrupted during cycles of heroin addiction. More recently, we have also shown disruption of the hypothalamic-pituitary-adrenal axis in recently abstinent cocaine addicts. Prolactin release is also disregulated in each of these addictive diseases, possibly reflecting derangement of normal opioid-dopamine modulation. We have developed an experimental protocol in which cocaine is administered to rats in a "binge" pattern, similar to that used by cocaine addicts; using this paradigm, we (Unterwald et al.) have shown that both mu and kappa opioid receptor density is significantly increased in the nucleus accumbens, caudate putamen. as well as some other brain regions which are part of the mesolimbic, mesocortical, or nigrostriatal dopaminergic systems. Using techniques of solution hybridization protection assay modified within our research Center (Inturrisi, Branch, Robertson et al.), we have re-examined the localization of opioid peptide gene expression in the brain, and have found that both enkephalin gene expression (Branch et al.) and prodynorphin gene expression (Spangler et al.) are abundant in the nucleus accumbens, and caudate putamen, and also in the hypothalamus, important for regulation of endocrine neuropeptide release.

Following the cloning of the specific opioid receptors and the availability of probes generously supplied by several collaborators (L. Yu, G. Uhl, C. Evans, B. Kieffer, and M. Abood), we have initiated studies in mapping of opioid receptor gene expression. We have found that there is abundant expression of the mu opioid receptors in the nucleus accumbens, caudate putamen, ventral tegmental area, substantia nigra, and hypothalamus, as well as in other areas where mu receptors have been previously identified by selective ligand binding to be very abundant; kappa opioid receptor mRNA has been found to be abundant in these areas (Spangler et al.). Our studies of the effects of cocaine on opioid peptide gene expression have shown that there are no persistent changes in enkephalin mRNA levels (Branch et al.) but increased expression of prodynorphin mRNA levels after chronic cocaine administration (Spangler et al.). In on-going studies, simultaneous measurement of dynorphin and kappa receptor mRNA in the same animal following binge pattern cocaine or saline administration is being determined; preliminary findings suggest there is a positive correlation between the increases in mRNA of both of these opioid system genes (Spangler et al.). In other on-going studies (Unterwald et al.), the effects of naltrexone on mu receptor mRNA levels has been studied, and to date, no alterations found despite significant "up-regulation" (i.e., increased density of mu opioid receptors. Also, preliminary studies of the effects of methadone on $\mathrm{mu}$ opioid gene expression (Unterwald et al.) have found no significant changes. Studies conducted in rodent models are being paralleled with studies using specific opioid receptor-directed ligands and the PET imaging techniques by our group in collaboration with the NIH to determine the effects of drugs of abuse and treatment agents in humans on the endogenous opioid system. 


\section{Quantitation and Localization of Opioid Receptor Gene Expression}

Charles E. Inturrisi, Department of Pharmacology, Cornell University Medical College, Ithaca, NY

We have developed a specific and sensitive solution hybridization assay for the delta opioid receptor (DOR) mRNAs using the cDNA of Evans et al., 1992. With Dr. V. Pickel we have prepared site directed antibodies to DOR so that we can determine both DOR receptor mRNA and protein levels. Exposure of neuroblastoma hybrid (NG108-15) cells to either naloxone or ethanol results in an increase in DOR transcripts. Image analysis of northern blots of the RNA extracts of control and treated NG 108-15 cells indicates that each of the six DOR mRNA transcripts is increased proportionately by ethanol or naloxone. The timecourse of this up-regulation differs for naloxone and ethanol and the inductive effects of these two drugs are at least addictive. Our results suggest that they upregulate DOR mRNA by independent mechanisms.

After six days of exposure to retinoic acid, DOR mRNA levels in NG108-15 cells were increased 3-fold. Retinoic acid treatment induced differentiation and when the cells are labeled with DOR antiserum we observed the presence of neurite-like processes with numerous branching patterns not seen in vehicle treated cells. These results suggest that the DOR gene may possess retinoic acid regulatory elements.

Steady-state DOR mRNA levels are not altered in mice made tolerant to the analgesic effects of the selective delta ${ }_{2}$ opioid receptor agonist, [D$\mathrm{Ala}^{2}$ ]Deltorphin II (DELT II), In contrast to NG108-15 cells which contain six different DOR mRNA transcripts, mouse brain RNA extracts contains only a single $10 \mathrm{~kb}$ DOR transcript which appears to be approximately the same size as the largest of the six DOR transcripts found in NG108-15 cells. In samples obtained by microdissection of CNS regions, DOR mRNA levels were highest in the caudate-putamen at $3.3 \pm .03 \mathrm{pg} / \mathrm{ug}$ RNA and lowest in cerebellum $(0.5$ $\mathrm{pg} / \mathrm{ug}$ RNA). Intermediate levels were observed in frontal cortex, nucleus accumbens, olfactory tubercule and medial thalamus. The DOR mRNA levels in these CNS regions of or DELT II tolerant mice did not differ significantly from control values. These data suggest that altered steady-state DOR mRNA levels are not one of the adaptive changes observed in mice tolerant to this delta opioid agonist.

ACKNOWLEDGEMENTS: All of the involved studies presented in this NIDA 20th Anniversary Symposium were supported in part by NIDA.

AFFILIATIONS: National Institutes on Drug Abuse, National Institutes of Health, Rockville, MD

Department of Pharmacology, University of Minnesota, St. Paul, MN

Department of Biochemistry, University of Minnesota, St. Paul, MN

Department of Biochemistry, Kon-Kuk University, Choongju-City, KOREA

Departments of Neurology and Neuroscience, Johns Hopkins University, Baltimore, Maryland

Department of Medical and Molecular Genetics, Indiana University, Purdue, IN Laboratory on the Biology of Addictive Diseases, The Rockefeller University, New York, New York

Department of Pharmacology, Cornell University Medical College, Ithaca, NY

Pharmacology Research Associate Program, NIGMS, NIH, Bethesda, MD

Department of Psychiatry and Biobehavioral Sciences, University of California, Los Angeles, CA 


\title{
HISTORICAL SUMMARY OF NIDA SUPPORTED OPIOID RESEARCH: A TRIBUTE TO NIDA ON ITS 20TH ANNIVERSARY
}

\author{
E. J. Simon
}

It is a real privilege to chair the symposium entitled, "Uptake on Opioid Receptor Cloning: Implications for Research on Addiction", together with my good friend, Mary Jeanne Kreek. The symposium was designated as a celebration of the 20th anniversary of NIDA and Mary Jeanne asked me to say a few words of a historical nature in honor of NIDA's birthday. I am honored and well qualified to perform this pleasant task, because I am NIDA's longest continuous grantee and have served on many of NIDA's committees as well as on its Advisory Council.

I want this brief talk to be a tribute to NIDA leadership and especially to the wisdom and courage they have shown in supporting basic research from the very beginning. It is not difficult to convince Congress of the need for basic research on oncogenes, cell division or metabolic pathways in the case of research on cancer or genetic diseases, but it takes enormous courage and foresight to advocate basic research for a condition like Drug Abuse, which, as you know, is not even considered a disease by most people, let alone a condition that may involve deficiencies at the cellular or molecular level.

NIDA's progress in treatment and prevention research was ably discussed by Dr. Alan Leshner, the new Director of NIDA, and by this year's winner of the Nathan B. Eddy Award, Dr. Jerome Jaffe. I plan to concentrate on the history of basic biochemical research on opiates. This clearly comes under the rubric of what Dr. Leshner called "sharing the secret". There are two good reasons why I shall limit myself to the opiates. First, it is the area I know something about, having been involved in it since the beginning of the modern era of this research and second, because it is the topic of this symposium. How well known is it that a brand new system of chemical signaling in the brain was discovered almost entirely by NIDA grantees? It is a much too well kept secret! This work also opened up the important and active field of neuropeptide research, which had lain dormant until the discovery of the endogenous opioid peptides.

We shall begin the story some decades before the birth of NIDA to pay tribute to the organic chemists and pharmacologists whose brilliant research laid the ground work. In their quest to find an effective, non-addictive analgesic, a typical applied research effort, these investigators made some very important fundamental discoveries: 1) the requirement of certain structural features for effective analgesia; 2) the stereoselectivity of analgesic action; and 3) the important finding that small changes, such as the replacement of the methyl group on the tertiary nitrogen of morphine and its congeners, leads to the production of effective opiate antagonists. Some of the names that should be mentioned in this connection are Pohl, Unna, Nathan B. Eddy, Harry Collier and some who are still with us, such as Everette May, Syd Archer, Eddie Way, Hans Kosterlitz, Albert Herz and others too numerous to mention, to whom I apologize. 
These discoveries gave rise to the receptor concept, the idea that these drugs must bind to specific sites in the brain in order to exert their pharmacological effects. As early as 1954, Beckett published his picture of what such a receptor might look like, based on the structures common to all effective opiate analgesics. This was followed by many attempts to demonstrate the existence of such receptors. Unsuccessful but nonetheless important contributions were made by Phil Portoghese and A. Takemori, N. Ingoglia, and V. Dole, and by A. Goldstein. Such an unsuccessful attempt was also contributed by Mrs. Van Praag and myself in 1971.

In this context, the contributions of Dr. Hans Kosterlitz must be mentioned. Their importance cannot be overstated. He took the guinea pig myenteric plexus, first developed by Paton and Schaumann, and improved it for use as a reliable bioassay and a model for the actions of opiates. He took considerable, usually good natured, ribbing about his preoccupation with "a piece of gut". However, nobody laughed when he and John Hughes used this piece of gut to discover the enkephalins. He then went on to develop several other bioassay systems (mouse, rat and rabbit vas deferens), which proved useful for determination and characterization of ligands of the multiple families of opioid receptors.

In 1973 three laboratories, Lars Tcrenius at Uppsala, Sweden, Candace Pert and Solomon Snyder at Johns Hopkins and my own laboratory at New York University, independently and simultaneously demonstrated the existence of stereospecific opiate binding sites in animal and human brain. This was quickly followed by the discovery of the endogenous opioid peptides. First, the enkephalins by Hughes and Kosterlitz, then the endorphins, independently by R. Guillemin and by Smyth and Bradbury, and, finally, the dynorphins by A. Goldstein and his collaborators.

To show you that no ideas are completely now under the sun, let me cite an astonishing statement made in the curly 19th century by Baudelaire, a brilliant writer and non-scientist: "Everyone carries within him his own morphine, which is constantly being secreted and renewed."

The speed with which the molecular genetics and biosynthesis of the opioid peptides was worked out is truly remarkable. This work was done by Nakanishi and the late S. Numa in Japan and by Sidney Udenfried, the late Ed Herbert and his students, Mains and Eipper, in the USA. We know a great deal about the structure and metabolism (biosynthesis, processing) of the three protein precursors, which account for all of the known peptides. We also know the structures of the genes that encode these proteins. They arc sufficiently similar to suggest the hypothesis that during evolution they were derived from a single gene-by-gene duplication.

The idea that there may be several types of opioid receptors had been in the air for a while, but it was the late Bill Martin who provided the first evidence. His work together with the discovery of $\delta$ receptors in the mouse vas deferens by Kosterlitz and co-workers, provided the evidence for the existence of three major opioid receptor families, $\mu, \delta$, and $\kappa$. There is evidence for subtypes of these as well as for other types of opioid receptors. However, these three types remain the best studied and best established ones.

The race to clone one or more of the opioid receptors started about six to eight years ago. All of the major opioid biochemistry laboratories, including my own, and many of the outstanding cloning laboratories all over the world 
(I counted 17 laboratories without really trying) spent much time and effort to achieve this goal, but without success. It was thus left to two young investigators, C. Evans, UCLA and B. Kieffer, University of Strasburg, France, one of whom (Evans) is speaking at today's Symposium, to solve this problem. They did it by using expression cloning techniques to clone and sequence the $\delta$ receptor from NG108-15 cells in culture. Once its sequence was known, low stringency screening with oligodeoxynucleotide probes, derived from the $\delta$ receptor cDNA structure, quickly led others to the sequences of the $\mu$ and $x$ types of opioid receptors. At the time this is being written, only one gene has been cloned for each of the three major receptor types, but subtypes will surely be found in the near future. Some of the people involved in this work (Lei Yu, George Uhl) are also represented here and we shall hear from all of the speakers that indeed "there is life after cloning".

This brings the story up-to-date with, of necessity, many omissions. I hope that it proves to the younger scientists the considerable foresight and great courage of the NIDA leadership in supporting first class fundamental neuroscience research related to the opiate drugs and that this policy has paid off in very important results. I want to end by welcoming Alan Leshner to his new and exciting position and compliment the search committee on its excellent choice. I wish NIDA well for the next 20 years and more. I am willing to make the prediction that the results I have reviewed, together with the important findings impact on our understanding of the underlying causes of drug abuse and of other mental disorders. They will undoubtedly lead to a more rational approach to treatment and prevention. I am certain that under the able leadership of Alan Leshner and Richard Millstein the wise policy of supporting basic research will be continued, the secret will be told, and NIDA will be one of the most respected members of the NIH community of Institutes.

AFFILIATION:

New York University, Medical Center, New York, NY 


\title{
DRUG ABUSE AND THE HEALTH OF WOMEN
}

\author{
L. P. Finnegan, Z. Sloboda, H. W. Haverkos, N. K. Mello, \\ M. J. Kreek, L. B. Cottler and D. A. Frank
}

Drug abuse among women has risen again as an important issue after an almost 20 -year hiatus. In the mid- 1970s, as a result of the women's movement, a great deal of interest in women drug abusers and their special needs, particularly relative to bearing children, spurred several meetings, articles and monographs summarizing the state of knowledge and specifying directions for new research. The dual epidemics of cocaine and crack abuse and of the human immunodeficiency virus and their differential and devastating effect on women, has lead to a resurgence of interest in women and drug abusing behaviors. In general, studies of deviant and antisocial behaviors have shown that prevalence rates are significantly lower for girls and women than for boys and men. However, these observations are changing, and certainly for substance abuse, women are beginning to show increasing rates of involvement. Women's substance abusing behaviors also have been of a more traditional nature-using alcohol or prescription medications thus avoiding the criminal distribution networks. And even when women do use more stigmatized drugs such as marijuana, cocaine, and heroin, they generally have received these drugs through their male significant other. Recent trends in our national and locally-based epidemiologic studies indicate that these trends appear to be changing; i.e., more women are using tobacco and marijuana and, in some cities (i.e., Atlanta), more women are involved at high levels, in the trafficking of drugs. It is not clear as to why these changes are taking place but there arc several hypotheses related to the changing role of women in this society and to the multiple generations of drug use in families. Current epidemiologic data on drug using behaviors among women have been reported. Drug use by women, as reported in the 1992 National Household Survey on Drug Abuse, for the 30 days prior to the interview was: 4.4 million women used an illicit substance; 3.1 million used marijuana; 0.4 million used cocaine. Lifetime prevalence of heroin use among women is estimated to be 1.0 million (1991). The percent use of cocaine in the past year was $1.8 \%$ white female, $2.7 \%$ black, $2.3 \%$ hispanic. Heroin use is difficult to ascertain. Marijuana past year use in women was 7.4\% white, $8.2 \%$ black, $5.0 \%$ hispanic. The 1992 National Client Data System women admissions was 411,839; 53\% are non-hispanic white; $29 \%$ are non-hispanic black; and $8 \%$ are hispanic.

Infectious diseases are commonly diagnosed and reported among female drug abusers. Severe communicable infectious diseases, such as HIV infection /AIDS, tuberculosis (TB), hepatitis B virus infection, and syphilis are common among women and amenable to intervention in drug abuse treatment settings. In fact, drug abuse treatment programs may be ideal sites to identify those infections and initiate and maintain appropriate medical management. AIDS is the newest, fastest growing, and most life-threatening of the four listed above. In the 1990's, AIDS is increasing twice as rapidly among women than among men; female injecting drug users and female sexual partners of injecting drug users are at especially high risk. Tuberculosis has re-emerged as an important pathogen in several parts of the USA, due, in part, to the HIV epidemic. Making an early diagnosis of active TB and ensuring compliance with TB treatment regimens will 
be instrumental in blunting the impact of $\mathrm{TB}$ in the drug using community. Hepatitis B virus infection is a common disease among drug users and can be prevented by Hepatitis B vaccination, which became available in the 1980's. Syphilis is making a resurgence in the U.S., due in part to the "crack" cocaine epidemic. Increases in congenital syphilis rates in some parts of the country are disturbing. Drug abuse treatment providers can be instrumental in battling these diseases among drug users by integrating services and follow-up with primary medical care and public health providers.

Studies have shown that several aspects of human physiology are significantly disrupted in the setting of drug abuse. These abnormalities include profound disruption of neuroendocrine function and possibly related disruption of specific indices of immune function. Both restoration of drug induced abnormalities of neurocndocrine function, and possibly related indices of immune function, as well as prevention of HIV infection is essential for women and men, and may be achieved. The endogenous opioid system, with the now defined three classes of endogenous opioid peptides, the endorphins, enkephalins, and dynorphins, and three types of opioid receptors, mu, delta, and kappa, is deranged in the setting of heroin addiction. Of a special importance is the opioid peptide precursor, proopiome-lanocortin (POMC), which yields both ACTH and beta-endorphin; the release of POMC neuropeptides, as reflected by circadian rhythm and plasma levels, is abnormal in the setting of heroin addiction. Studies on the HPA axis in our laboratory and others have determined that, in addition to the well established control of POMC peptide release by CRF and the negative feedback control by cortisol, that the endogenous opioids, acting at mu and possibly also kappa opioid receptors, are involved in the normal tonic regulation of the synthesis-release of the POMC peptides. In humans, acute use of any opiate, and chronic use of any short-acting opiate such as heroin or morphine, causes suppression of the HPA axis; conversely, in the setting of opiate withdrawal, (or following administration of an opioid antagonist to an opiate naive subject) activation of the HPA axis is observed. During long-term methadone maintenance treatment, normalization of this important axis occurs. The responsivity of the HP axis to chemically induce stress has also been shown by our laboratory to be abnormal in the setting of opiate dependency with suppression of responsivity. This response is restored to normal in the setting of chronic methadone maintenance treatment. However, it has recently been found to be hyper-responsive in both drug-free former opiate addicts and recent abstinent cocaine addicts.

Immune function also is profoundly disrupted in heroin addicts. This may be due in large part to injection of foreign substances, infection with multiple diseases, and lifestyle. However, laboratory models have shown that the endogenous opioid system may be involved in normal regulation of the endogenous opioid system, and thus exogenous opioids may contribute to the derangements observed. Studies have found that whereas there were significant abnormalities of absolute numbers of CD4 and CD8 cell numbers, levels of immunoglobulin G and $\mathrm{M}$, and of special importance, a natural reduction of natural killer cell activity in heroin addicts, each of these indices normalize during long-term methadone maintenance treatment. Further studies are ongoing using techniques of molecular biology and imaging to study the derangements of the endogenous opioid system in the setting of addiction, and the changes which occur during specific pharmacotherapies. Many of these findings are of special importance to women with respect to the effects of the endogenous opioids in the normal modulation of prolactin release, exogenous opioids in abnormal disruption of prolactin release, as well as of the peptides of the hypothalamic-pituitary-gonadal axis. 
Alcohol and cocaine abuse each are associated with a series of reproductive disorders including amenorrhea, luteal phase dysfunction, anovulation and hyperprolactinemia. The way in which abused drugs affect the regulation of the hypothalamic pituitary ovarian axis is poorly understood. The increasing prevalence of polydrug abuse further complicates efforts to analyze the effects of individual drugs in clinical studies. Fortunately, neuroendocrine control of the menstrual cycle in rhesus monkey is very similar to that of women, and the effects of drugs on the endocrine system can be studied in the primate model of drug selfadministration. One of the major recent findings to emerge from both clinical and primate studies is that acute alcohol and cocaine intoxication can increase rather than suppress hormones important for a normal reproductive function. For example, alcohol stimulates estradiol under basal (non-stimulated) conditions as well as after luteinizing hormone releasing hormone and opioid antagonist stimulation. High estradiol during the follicular phase may disrupt folliculogenesis by decreasing $\mathrm{FSH}$ which in turn may lead to luteal phase defects. An increase in estradiol during the luteal phase could result in luteal phase defects by a direct suppressive effect on progesterone. Both cocaine and alcohol stimulate ACTH and by inference, corticotrophin releasing hormone (CRH). Synthetic $\mathrm{CRH}$ administration suppresses gonadotropin release which may result in anovulation and amenorrhea. Cocaine also stimulates luteinizing hormone which could lead to premature ovulation. In summary, either the acute stimulatory effects of alcohol or cocaine intoxication on gonadotropins, estradiol or ACTH or the suppressive effects of chronic intoxication may disrupt the intricate functional integration of the endocrine system and lead to infertility disorders and menstrual cycle disruption.

In reviewing the psychosocial risk factors associated with female drug use, the characteristics of St. Louis female drug abusers from two NIDA-funded projects conducted from 1990 to 1994 are described. The Substance Abuse and Risk for AIDS study aimed to determine risk factors for HIV infection and comorbidity of psychiatric and substance use disorders among recent admissions to drug treatment. Baseline and 12-month follow-up interviews were conducted. The Efforts to Reduce the Spread of AIDS studied out-of-treatment drug abusers and focused on risk factors for HIV. Abusers were offered free drug treatment and those who accepted the treatment and those who didn't were interviewed (at baseline $3,6,9,12$, and 18 months). Women represented over a third of the sample with $75 \%$ minority representation. Women recruited for these two projects were stratified into two groups, those who had injected drugs $(\mathrm{N}=160)$ and noninjectors $(\mathrm{N}=221)$. Injectors were more likely than non-injectors to have: a family background of social disorganization; report alcoholism among siblings; higher rate of substance dependence; higher rates of most medical problems, including pneumonia, difficulty getting pregnant, and hepatitis B; psychiatric disorders, especially comorbid ASPD and depression; higher rates of risky sexual behavior; history of drug treatment and recent life events. In another comparison made between women and men, women were more likely to have reported barriers to drug abuse treatment. The implications of these data with regard to assess to mental health services, life events issues and barriers to care are most relevant for women desiring treatment.

The impact upon children born to drug using women has been a source of concern to many. It has been nine years since a report in the New England Journal of Medicine suggested that a mother's cocaine use during pregnancy was associated with important adverse effects on the newborn. Subsequently, the lay press led a public rush to judgement, labelling children with a history of prenatal cocaine exposure as "crack kids," inevitably and permanently damaged, with 
developmental and behavioral deficits so profound as to jeopardize the public school system. The peer reviewed scientific literature regarding the potential association of in utero cocaine exposure with perinatal deficit and less optimal developmental outcome in infancy and childhood is much less conclusive than the dire quasi-scientific predictions in the popular press would lead one to believe. Many complex methodological issues contribute to scientific uncertainty regarding the possible effects of prenatal cocaine exposure. These include difficulties in accurate identification of users, uncertain measurement of dose and gestational timing of exposure, sample selection bias, failure to control for confounding variables, lack of appropriate comparison populations, and selection of sensitive outcome measures. In spite of these difficulties, there is a growing body of relatively credible data to suggest that prenatal cocaine exposure is independently associated with increased risk of delivering smaller infants, whether from decreased gestation, intrauterine growth retardation, or both. However, it is not clear whether cocaine imposes long term developmental risks in excess of that associated with prematurity and IUGR. Cocaine may have subtle behavioral effects, not obvious on global test scores. Moreover, poor care-giving environment may explain in part poor developmental outcome. Compensatory responsive environments significantly improve the outcome of infants with biological vulnerabilities at birth.

Scientists and clinicians should never confound issues of legality or morality with those of toxicity; using infant meconium and maternal self-report, it is necessary to assess issues of dose and thresholds of toxicity for all psychoactive substances, legal or not. Furthermore, it will be important to delineate which biologic and social factors together identify infants with the greatest burden of risk following cocaine exposure. Future research will focus on linking central nervous system findings to developmental outcome in cocaine exposed infants. These findings include not only obvious hypoxic/vascular lesions such as IVH, but less visible CNS neurotransmitter alterations. Outcome measures at later ages need to include tasks that assess the potential effects of such perturbations, particularly in areas such as the frontal lobe, which are rich in dopaminergic projections. These include social competence, and regulation of arousal and attention to global developmental scores. Much more well controlled longitudinal research remains to be done if the myth of the demonic "crack baby" is to be replaced by high quality scientific data that will facilitate rational and effective care of vulnerable women and children.

\section{REFERENCES:}

Furnished upon request of Senior Author.

\section{AFFILIATIONS:}

National Institute on Drug Abuse, National Institutes of Health, Rockville, MD Rockefeller University, New York, NY McLean Hospital, Harvard Medical School, Cambridge, MA Washington University School of Medicine, St. Louis, MO Boston City Hospital, Boston, MA 


\title{
AFFECTIVE DISORDERS AND SUBSTANCE ABUSE: FROM BENCH TO BEDSIDE AND BACK
}

\author{
T. R. Kosten, E. Gardner, A. Markou, R. Pickens and F. Goodwin
}

The first presentation was entitled, "COMMON GENETIC MECHANISMS IN DEPRESSION AND SUBSTANCE ABUSE," by R. W. Pickens from the NIDA Addiction Research Center. This presentation began by reviewing the general epidemiology of substance abuse and depression. Major depression occurs more often in alcoholic individuals and their first degree relatives than is expected by chance. To determine if the comorbidity between alcoholism and depression is due in part to common genetic influences, within and crosscorrelation for the disorders were examined in monzygotic (MZ) and dizygotic (DZ) twins. In 130 twin pairs, at last one twin met criteria for DSM-III alcohol dependence. Results suggest that co-occurrence of certain mental disorders in alcoholics and their families observed in epidemiological surveys may have a common genetic basis. Specifically, higher MZ than DZ cross-correlation was observed between alcohol dependence in male probands and antisocial personality disorder and phobia in the co-twins. For males, correlation between alcoholism and depression was .51 within probands; correlation between alcoholism in the proband and depression in the co-twin was .54 for MZ twins and .32 for DZ twins. For females, within-proband correlation between alcoholism and depression for proband and co-twin was .18 for $\mathrm{MZ}$ and .20 for DZ twins.

These results were not a consequence of correlation for antisocial personality or phobia that may have also existed in the twins nor were they due to comorbid alcohol dependence in the co-twin. Cross-correlation between alcohol dependence in the proband and certain drug disorders in the co-twin was attributable to environmental factors. Additional analysts indicated that alcohol dependence may be more heritable when the proband has a comorbid drug or mental disorder, at least for males. Probands with a comorbid drug disorder were significantly younger and reported significantly earlier ages of first intoxication and alcohol problems than probands without a comorbid drug disorder. While individuals with comorbid drug disorder exhibited more alcoholism symptoms, they did not differ in quantity or frequency of alcohol consumption. These results suggest common genetic influences may underlie comorbidity between depression and alcoholism, at least for males. The results suggest common genetic influences may underlie comorbidity between depression and alcoholism, at least for males. The results also suggest that the observed association between alcoholism and depression in females may be due to environmental influences.

The second presentation by T. R. Kosten from Yale University was entitled, "CLINICAL STUDIES ON THE RELATIONSHIP OF AFFECTIVE DISORDERS TO SUBSTANCE ABUSE.” Since 1935, substantial increases in both depression and substance abuse have been observed, particularly in younger individuals. The reasons for increases in substance abuse have included changes in public attitudes, increased availability and new more 
addictive forms of abusable drugs. The reasons for more depression in younger patients are less clear, but in recent studies of adolescents, the rates of depression have been three times higher in substance abusers vs non-abusers $(25 \%$ vs $8 \%)$. The additional $17 \%$ of depression has begun after the development of substance dependence.

Many drugs may have direct toxic effects on mood. For example, chronic use of depressants such as alcohol and barbiturates leads to dysphoria, and withdrawal from stimulants such as cocaine is characterized by anhedonia. Opioids such as heroin or methadone do not appear to produce mood disruptions, yet the rates of depression are about seven-fold higher in opioid dependent patients than in the general population. Thus, opioid dependence provides a most interesting disorder for examining this association. Furthermore, neurobiological comparisons of depressives to substance abusers using the dexamethasone suppression test, the Thyrotropin releasing hormone stimulation test or CSF levels of dopamine metabolites show striking similarities between these patient groups.

The clinical association between these disorders includes not only high rates of disorder, but differences in treatment response. Depressed substance abusers usually have poor treatment responses to standard substance abuse treatments such as detoxification from nicotine, alcohol or opiates, but these patients respond well to antidepressants not only in symptom reduction, but also in reduced substance abuse. This reduction in substance abuse is less robust than the depressive symptom response, but it has been most clearly shown in methadone maintained samples. This stronger demonstration in methadone patients is probably because depression in these patients is less likely to result from toxic effects of opioids on mood.

The third presentation by Gardner from Einstein University was entitled, "COVULNERABIITY BETWEEN AFFECTIVE DISORDERS AND SUBSTANCE ABUSE: PRECLINICAL PERSPECTIVES." Animal models of drug/alcohol abuse include: a) voluntary intravenous drug self-administration; b) voluntary intracerebral drug self-administration; c) voluntary electrical brainstimulation reward, and its modulation by drugs/alcohol; and d) conditioned cue preference to alcohol/drugs. Using these models, it has been established that drugs of abuse acutely facilitate the functioning of brain pleasure/reward circuits, producing the euphoric "high" or "rush" that the drug user seeks. It has also been established that the critical drug-sensitive locus of the brain's pleasure/reward circuits involves a) mesocorticolimbic dopaminergic (DA) neurons arising from the ventral tegmental area, projecting through the medial forebrain bundle, and synapsing in the nucleus accumbens, ventral striatum, and medial prefrontal cortex; and b) endogenous opioid neurons interconnecting synaptically with, and exerting modulatory control over, the mesocorticolimbic DA pleasure/reward neurons. These endogenous opioid neurons appear crucially involved in drug/alcohol abuse, since they appear to set the "gain" or range-of-function of the DA pleasure/reward substrates, and since the effects of drugs of abuse on these brain substrates are naloxone blockable. Also, naloxone and naltrexone are effective treatments for craving of some drugs abused by humans and attenuate free-choice drug consumption in addicted laboratory monkeys. Thus, aberrations in brain opioid systems may constitute a neurobiological vulnerability.

Animal models of depression include, most compellingly, "learned helplessness" - an animal behavioral syndrome produced by inescapable shock 
which includes symptoms of human depression, including weight loss, lethargy, anergy, sleep disturbance, poor grooming, diminished libido, seasonal variation, and favorable response to antidepressant drugs. Opiate antagonists (naloxone, naltrexone) attenuate learned helplessness. Attenuation is also seen with intracerebroventricular injections of quaternary naltrexone, implicating central opioid mechanisms. Learned helpless animals also show reduced naloxoneprecipitated morphine withdrawal, reduced plasma $\beta$-endorphin levels, and increased $\mathrm{mu}$ opiate receptors in limbic brain regions as compared to nonlearned helpless or naive control rats. Thus, aberrations in brain opioid systems may constitute a neurobiological vulnerability to learned helplessness.

Another common neurobiological vulnerability between drug/alcohol dependence and learned helplessness may derive from scrotonergic (5HT) innervation and regulation of the mesotelencephalic DA pleasure/reward circuitry activated by drugs/alcohol. That this 5HT-mediated regulation of forebrain DA pleasure/reward neurons may have functional relevance for drug/alcohol dependence is suggested by observations that 5HT-specific reuptake inhibitors reduce self-administration of cocaine, amphetamines, and alcohol in laboratory animals, reduce alcohol preference in animals, and reduce the progressive-ratio break-point for intravenous cocaine reinforcement in animals. In humans, 5HT-specific reuptake inhibitors reduce some alcoholcocaine consumption and craving. Aberrations in 5HT function have been hypothesized for decades to constitute a neurobiological substrate of depression in humans, and 5HT-specific reuptake inhibitors are therapeutic for human depression. Provocatively, significant alterations in 5HT functions in limbic brain areas have been demonstrated in learned helplessness. Thus, aberrations in endogenous brain 5HT systems regulating and modulating mesotelencephalic DA pleasure/reward circuitry may constitute a neurobiological vulnerability to drug and/or alcohol dependence and to learned helplessness.

The final presentation by Markou from the Department of Neuropharmacology at The Scripps Research Institute was entitled, "ELEVATIONS IN REWARD THRESHOLDS AS AN ANIMAL MODEL OF AFFECTIVE DISORDERS." This presentation focused on one of the major symptoms of affective disorders "anhedonia", which is defined as "the markedly diminished interest or pleasure in all, or almost all, activities most of the day, nearly every day" (DSM-III-R). Because the "anhedonia" experienced by depressed patients suggests that these individuals exhibit alterations in reward processes, the reward thresholds provided by the intracranial self-stimulation (ICSS) paradigm provide an operational measure of this core symptom of depression.. Investigations of brain stimulation reward without any prior manipulations have not provided a satisfactory model of depression because, a) the study of reward processes in "normal" organisms, although informative, does not mimic any aspect of depression; and b) acute or chronic treatments with tricyclic antidepressants do not affect dramatically baseline ICSS behavior. In contrast, the study of the neuro-substrates of ICSS behavior following pharmacological manipulations promises to promote our understanding of reward mechanisms that seem to be altered in several psychiatric disorders including depression and schizophrenia (DSM-III-R).

A pharmacological manipulation that has been used to product elevations in ICSS reward thresholds in rats is withdrawal from long-term exposure to several drugs of abuse, such as psychomotor stimulants, opiates and ethanol. Rats allowed to self-administer cocaine for prolonged periods of time $(3,6,12,24$ or 
48 hours) showed a marked elevation in ICSS thresholds. The magnitude and duration of this effect was proportional to the amount of cocaine consumed during the preceding self-administration episode ("binge") which was a function of the duration of the self-administration session. During the time-points when maximal elevations in thresholds were observed, dialysate dopamine levels in the nucleus accumbens, as measured by in vivo microdialysis, were decreased compared to pre-"binge" dopamine levels. Similarly to the threshold elevation, the reduction in dopamine levels was proportional to the duration of the preceding self-administration session.

Pretreatment with bromocriptine, a dopamine agonist, reversed the post-cocaine elevation in thresholds in control subjects. Furthermore, chronic treatment with desmethylimipramine (a tricyclic antidepressant), at a dose that produced a significant down-regulation of beta-adrenergic receptors, had no effect on the severity of this withdrawal symptom during the first three hours post-cocaine, but significantly shortened the duration of withdrawal. These results implicate a role of dopaminergic and noradrenergic neurotransmission in the affective aspects of cocaine withdrawal. In addition, the effectiveness of an antidepressant in reversing the post-cocaine elevation in thresholds suggests a potentially common neurochemical abnormality underlying both drug and nondrug induced depressions.

Similar elevations in reward thresholds were also observed during naloxoneprecipitated opiate withdrawal in rats made dependent through the subcutaneous implantation of morphine pellets. Finally, rats made dependent on ethanol through exposure of ethanol vapor for two weeks also exhibited time-dependent elevations in reward thresholds upon cessation of the ethanol vapors.

In conclusion, these results suggest that post-drug elevations in reward thresholds are a reliable operational measure of the "affective" aspects of withdrawal from several drugs of abuse, such as stimulants, opiates and ethanol. Even though it is not known at this point what the exact relationship is between drug-induced and non-drug-induced depression in humans, post-drug depression in both animals and humans may provide a valuable tool in the investigation of the neurobiology of affective disorders.

\section{AFFILIATIONS:}

Yale University School of Medicine

New Haven, Connecticut

NIDA Addiction Research Center, Baltimore, Maryland

Albert Einstein College of Medicine, New York, New York

The Scripps Research Institute, La Jolla, California

National Institute of Mental Health, National Institutes of Health, Rockville, Maryland 
YOUNG INVESTIGATORS' SYMPOSIUM:

\title{
ENVIRONMENTAL MODULATION OF DRUG EFFECTS
}

\author{
J. B. Kamien, L. Amass, S. L. Vrana, M. A. Nader, C. R. Bush and \\ R. W. Foltin
}

Impressive advances in neuroscience and molecular pharmacology sometimes overshadow the role of the environment in the modulation, and even determination, of a drug's behavioral effects. However, "it is a cardinal principle of behavioral pharmacology that the effects of drugs cannot be understood independently of the conditions under which the drugs are given and the previous drug history of the organism" (Schuster and Balster, 1977). This symposium highlights evidence of substantial environmental modulation of drug effects in five major areas of drug abuse research.

\section{ENVIRONMENTAL DETERMINANTS OF THE NEUROCHEMICAL EFFECTS OF DRUGS}

\section{Sheila L. Vrana}

The context of drug administration can alter the neurochemical effects of that drug. The yoked-box (triad) procedure focuses on the effects of responsedependent vs. response-independent administration of drugs of abuse (Smith, et al., 1982, 1984). In this paradigm, one animal self-administered (SA) morphine, while litter mates received either a response-independent infusion of morphine (yoked-morphine; YM) or saline (yoked-saline; YS). Using this procedure, the direct pharmacological effects of a drug can be evaluated (YM vs. YS) and compared with the reinforcing actions of the drug (SA vs. YM). SA morphine increased dopamine (DA) and acetylcholine (ACh) turnover in the frontal cortex, and decreased DA, 5-HT and ACh turnover in the nucleus accumbens (NAcc) as compared to YM. The same procedure has been used to examine the effects of response-dependent vs. response-independent administration of cocaine $(0.33 \mathrm{mg}$ /infusion; 80 infusions/day limit). This study found that $38 \%$ of the animals receiving response-independent cocaine died, while none of the rats receiving response-dependent cocaine or YS rats died. We have also used the triad paradigm, to examine the effects of response-dependent vs. responseindependent cocaine administration on tyrosine hydroxylase (TH) and mRNA levels. Response-independent cocaine increased $\mathrm{TH}$ activity and mRNA levels in the VTA and activity levels in the NAcc compared to when the animals selfadministered the drug.

Cocaine and heroin also increase extracellular dopamine $\left(\mathrm{DA}_{\mathrm{ec}}\right)$ in the NAcc (Pettit and Justice, 1989). The triad paradigm has been used to assess contextual effects on dopamine concentrations in the NAcc using in vivo microdialysis during cocaine self-administration (Hemby et al., 1992). DA $\mathrm{ec}_{\text {c }}$ increased significantly $(300 \%)$ in the SA compared to YC conditions, while there was no difference in NAcc cocaine concentrations. When the same pattern of cocaine infusions was delivered the next day to the SA animal in a response-independent manner, $\mathrm{DA}_{\mathrm{ec}}$ was no different than the $\mathrm{YC}$ pattern from the previous day. Hemby et al., (1993) also examined the effects of acute administration of 
heroin vs. chronic self-administration of heroin on $\mathrm{DA}_{\mathrm{ec}}$ in the NAcc. An acute i.v. dose of heroin $\left(30 \mu \mathrm{g}\right.$ /infusion) in a naive rat significantly increased $\mathrm{DA}_{\mathrm{ec}}$ by $180 \%$. However, in rats trained to self-administer 18 or $30 \mu \mathrm{g} /$ infusion, NAcc $D_{\text {ec }}$ was decreased by $20 \%$ and $50 \%$, respectively. These results indicate that there are neurochemical and molecular differences associated with the environmental context of drug administration.

\section{ENVIRONMENTAL MODULATION OF THE DISCRIMINATIVE STIMULUS EFFECTS OF DRUGS}

\section{Jonathan B. Kamien}

Drug discrimination (DD) results are commonly used to make pharmacological comparisons between drugs. However, the impact of the environment on the discriminative stimulus (DS) effects of drugs, although large, is often not considered. Examples of environmental modulation of the DS effects of drugs can be grouped into four categories: Schedules of Reinforcement, Response Options, Exteroceptive Stimuli and Historical Influences.

Relationships among the DS of drugs can be modulated by arranging unequal schedules of reinforcement for drug or saline responding. For example, pigeons were trained to discriminate PCP vs. saline and tested with PCP, pentobarbital (PB) and $d$-amphetamine. When the reinforcement schedules were equal (i.e., no bias), only PCP and PB substituted for PCP. When more responses were required on the PCP lever (i.e., bias towards saline), PB no longer substituted for PCP and the PCP dose-effect curve was shifted to the right. But when more responses were required on the saline lever (i.e., bias towards PCP), PCP, PB and amphetamine all occasioned PCP responding and the PCP dose-effect curve was shifted to the left (McMillan and Wenger, 1984). Another experiment compared morphine DD under a FI 180-s and a FR 100 schedule of reinforcement. Under the FI 180-s schedule there was much more individual variation. Also, responding under the FR 100 schedule described a quantal relationship between dose and morphine responding while responding under the FI 180-s schedule described a more graded curve (Massey et al., 1992).

The number of stimulus-response relations trained can determine the outcome of DD generalization tests. In one example, butorphanol occasioned a maximum of about $40 \%$ hydromorphone responding when humans were trained to discriminate among saline, hydromorphone and pentazocine (Preston et al., 1989). However, butorphanol occasioned nearly $100 \%$ hydromorphone responding when humans were trained to discriminate only hydromorphone from saline, (Preston et al., 1992). In another example, humans were trained to discriminate triazolam from placebo. When the only response options were triazolam or placebo, amphetamine occasioned about $25 \%$ triazolam responding. When the response options included a response for a 'novel' stimulus, $d$ amphetamine occasioned nearly 100\% novel responding (Bickel et al., 1993).

Exteroceptive stimuli present during training and testing can influence or even mimic the DS effects of drugs. Rats were trained to discriminate PB in darkness and saline in light. The PB dose effect curve determined in light was shifted to the right relative to when it was determined in darkness (Jarbe et al., 1983). Another experiment demonstrated that an exteroceptive stimulus (exposure to a cat) can substitute for pentylenetetrazol (PTZ) in rats trained to discriminate PTZ from saline (Gauvin and Holloway, 1991). Behavioral and drug history can 
also alter the DS effects of drugs. Baboons were trained to discriminate midazolam (MDZ). After the baboons self-administered MDZ, they were more sensitive to the MDZ DS. However, when they received the same amount and pattern of MDZ injections non-contingently, they were less sensitive to the MDZ DS (Ator and Griffiths, 1993).

\section{ENVIRONMENTAL MODULATION OF THE REINFORCING EFFECTS OF DRUGS.}

\section{Michael A. Nader}

Behavioral or environmental factors can influence the rate and pattern of drug self-administration. For example, the schedule of reinforcement has profound effects on drug self-administration rates. Identification in animals of conditions under which drug-seeking behavior could be reduced for extended periods might have direct practical applications. In the present review, two environmental variables will be discussed: behavioral history and punishment contingencies.

Behavioral History Exposure to certain reinforcement schedules can produce significant and long-lasting changes in the behavioral effects of drugs (see Nader et al., 1992). Recently, we have begun to systematically study the influence of behavioral history on drug self-administration. In one study, rhesus monkeys were initially trained to self-administer cocaine $0.03 \mathrm{mg} / \mathrm{kg} / \mathrm{inj}$ under a fixed-interval 5-min schedule (FI), before training under a schedule that generated high rates (fixed-ratio 50; FR) or low rates (inter-response-times $>30$ sec; IRT) of cocaine-maintained responding. When responding was again maintained under the FI schedule, response rates were significantly higher after an FR history than after an IRT history. In another study, a history of foodmaintained responding under either an FR or IRT schedule resulted in significantly different rates of cocaine self-administration. Thus, past environmental conditions influenced the rates at which animals acquired and maintained cocaine self-administration.

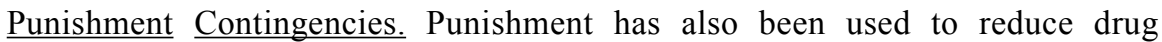
self-administration by animals. Punishment is the reduction in the probability of a response following either the presentation ("positive" punishment) or the removal ("negative" punishment) of a stimulus. Although negative punishment is more analogous to treatment programs that remove individuals from environments in which drugs are available, most punishment studies on drug self-administration have used positive punishment. Johanson (1977) used a discrete-trials procedure in which rhesus monkeys were given a choice between a dose of i.v. cocaine paired with an electric shock and one not paired with shock. If the doses were the same, monkeys preferred the cocaine dose not associated with punishment. However, increasing the cocaine dose associated with shock reversed this preference. Bergman and Johanson (1981) reported that intermediate shock intensities only transiently decreased cocaine selfadministration, suggesting that positive punishment is not an effective method for maintaining decreases in cocaine self-administration. We have begun studies on the effects of negative punishment on cocaine self-administration. Four rhesus monkeys were trained under a multiple FI 5-min schedule of cocaine presentation (0.01-0.3 $\mathrm{mg} / \mathrm{kg} / \mathrm{inj})$. The same dose was always available in both components. When responding was stable, the schedule in the second component was changed to a conjoint FI 5-min cocaine, VI 30-sec time-out schedule. Responding in the second component was suppressed by response- 
contingent time-outs of 10-90 sec durations. In addition, increases in cocaine dose did not attenuate the effects of negative punishment.

\section{ENVIRONMENTAL MODULATION OF SELF-REPORTED EFFECTS OF DRUGS}

\section{Craig R. Rush}

Self-report instruments (e.g., Addiction Research Center Inventory, Profile of Mood States [POMS]) are used extensively to assess drug effects in humans. The present paper briefly discusses environmental variables that modulate the self-reported effects of commonly used/abused drugs including: (1) the social context in which the drug is administered and (2) the type of task the subject completes after drug administration.

The self-reported effects of alcohol and marijuana can differ qualitatively and quantitatively depending on the social context (i.e., group vs. isolated setting) in which the drug is administered. Alcohol, for example, increased self-reported "elation" in a group setting, while it decreased these ratings in an isolated setting (Del Porto and Masur, 1984). Similarly, the "euphoric" effects of smoked marijuana were greater in a group vs. isolated setting, while the "dysphoric" effects of marijuana were less in a group vs. isolated setting (Jones, 1971). In a group setting most subjects reported the overall effect of alcohol and marijuana as "pleasant," while in an isolated setting most subjects reported the overall drug effect as "indifferent."

The type of task the subject is required to complete after drug administration also influences the self-reported effects of drugs (e.g., alcohol; O'Malley and Maisto, 1984). Following the administration of placebo, subjects who read a magazine reported less intoxication than subjects who played a video game. After a high dose of alcohol, however, subjects who read a magazine reported greater intoxication than subjects who played a video game. Similarly, the number of response options in a drug discrimination procedure influences the self-reported effects of drugs. When three (i.e., triazolam, placebo and novel) vs. two (i.e., triazolam and placebo) responses were available, subjects reported greater drug effects following low and intermediate doses of $d$-amphetamine and triazolam (Bickel et al., 1993).

In conclusion, environmental factors influence the self-reported effects of commonly used/abused drugs, a finding consistent with drug effects on other types of discriminated-operant behavior. Recognition of the influence of environmental factors on self-reported drug effects will help refine the use of these instruments in understanding the human behavioral pharmacology of abused drugs.

\section{ENVIRONMENTAL MODULATION OF DRUG EFFECTS IN DRUG ABUSE TREATMENT}

\section{Leslie Amass}

The effectiveness of pharmacotherapy for drug abuse is not only mediated by pharmacological effects. Treatment agents also serve behavioral functions and these functions can be modified by the environment. Better recognition of 
environmental factors can lead to more effective use of pharmacotherapy for drug abuse and help address three problems: compliance, retention and clinical effectiveness. Three environmental manipulations used to address these problems are contingency management, psychosocial interventions and instructions. How these manipulations interact with pharmacotherapy depend largely on whether the treatment agent functions as a reinforcer.

Disulfiram and naltrexone, which generally do not function as reinforcers, are associated with poor compliance and treatment retention, and equivocal clinical effectiveness. Contingency management procedures and psychosocial interventions have been useful in addressing these problems. For example, compliance with disulfiram in alcoholic methadone patients was increased by making methadone contingent on disulfiram consumption (Bickel et al., 1989). This procedure decreased drinking and increased treatment retention. Similarly, contingent monetary payments and the schedule used for those payments increased naltrexone compliance in recently detoxified opiate addicts (Grabowski et al., 1979).

Behavioral therapies also improved the effectiveness of disulfiram with alcoholics (Azrin et al., 1982) and naltrexone with opioid addicts (Callahan et al. (1980). Use of these behavioral treatments increased compliance and improved lifestyle relative to traditional psychotherapy.

Methadone, buprenorphine and nicotine gum, which generally function as reinforcers, present less of a problem with compliance and retention, but their overall clinical effectiveness can be enhanced by non-pharmacological factors. For example, greater reduction in illicit drug use during methadone or buprenorphine detoxification occurred when cash payments (Hall et al., 1979) or vouchers (Bickel et al., 1993) were contingent on opioid-free urines. Further, the level of counseling services provided was directly related to the efficacy of methadone therapy with opiate dependent patients (McLellan et al., 1993) and nicotine therapy with smokers trying to quit (Fiore et al., 1994).

Finally, instructions also modulate therapeutic effects. Providing patients with specific withdrawal information during methadone detoxification decreased withdrawal symptoms (Green and Gossop, 1988). In addition, placebocontrolled studies demonstrated that compliance, abstinence rates and selfreported effects in smokers trying to quit varied according to whether nicotine gum administration was instructed or blind (Hughes et al., 1989). Thus, instructions can modify therapeutic effects, and play an important role in pharmacotherapy.

\section{REFERENCES}

A reference list is available on request from Jonathan B. Kamien.

\section{AFFILIATIONS}

Bowman Gray School of Medicine, Winston-Salem, NC 27157

University of Vermont College of Medicine, Burlington, VT 05401

Bowman Gray School of Medicine, Winston-Salem, NC 27157

The University of Mississippi Medical Center, Jackson, MS 39216

University of Vermont College of Medicine, Burlington, VT 05401

College of Physicians and Surgeons of Columbia University

New York, New York 10032 


\title{
BRAIN IMAGING IN SUBSTANCE ABUSE
}

\author{
C. P. O'Brien and A. R. Childress
}

Advances in imaging technology over the last decade now enable direct, in vivo measure of the brain correlates of drug use and dependence in humans. In this symposium, investigators from five imaging centers presented their addiction-related work featuring PET (positron emission tomography), SPECT (single photon emission computed tomography), EEG (electroencephalography) and MRI (magnetic resonance imaging) technologies.

\section{BRAIN METABOLISM IN SUBSTANCE ABUSERS}

\section{Edythe London}

Dr. London described research using PET to study the regional metabolism of brain glucose during actual administration of several drugs of abuse, including opiates, cocaine, benzodiazepines, alcohol computed tomography) EEG (electroencephalography) and MRI (maand delta-9 THC (the active ingredient in marijuana). These studies have generally focused upon cerebral substrates for "euphoria", the positive affective state produced by drugs of abuse. PET studies using ${ }^{18}$ FDG to measure brain metabolism show that opioids, stimulants, benzodiazepines, and ethanol reduce cerebral glucose utilization. In contrast, delta-9-tetrahydrocannabinol stimulates glucose metabolism in the cerebellum. In studies with morphine and cocaine, reduced glucose utilization accompanies self-reports of euphoria. The effect on positive mood is related negatively to glucose metabolism in the temporal cortex. Therefore, function in this brain region appears to be a determinant of subjective responses to abused drugs that differ in their spectrum of action, but share the property of producing euphoria. Effects of abused drugs on cerebral metabolism may reflect a common neurochemical action. Studies in animals have suggested that drugs of abuse enhance dopaminergic neurotransmission in the mesolimbic reward system. Thus, drugs of abuse may reduce cerebral metabolism through dopaminergic activation.

\section{FLUORO-DOPA STUDIES OF HUMAN AND ANIMAL COCAINE ABUSE}

\section{William Melega}

Dr. Melega discussed PET imaging of uptake of a radioactively labeled form of the dopamine precursor, L-DOPA, in those exposed to chronic stimulants. Work with his colleague, Dr. Lewis Baxter, has previously demonstrated reduced uptake of ${ }^{18}$ F-fluoro-L-dopa (FDOPA) in human cocaine users, suggestive of reduced dopamine (DA) synthesis. Reduced synthesis may occur compensatory to the increased synaptic DA associated with cocaine's 
actions (reuptake inhibition of DA). In recent work with monkeys, timedependent decrements in activity of the striatal dopamine system were detected following ten days of amphetamine administration. At one month post -amphetamine, FDOPA uptake was reduced by $75 \%$ relative to pre-drug values, indicative of long-term dopamine depletion. At five months postamphetamine, FDOPA uptake was reduced by only $45 \%$, indicative of partial recovery of the dopamine system. Acute administration of low dose amphetamine effected a persistent loss of FDOPA uptake only when the monkeys were pretreated with chronic cocaine. Both the findings in humans, and the parametric studies in monkeys with known dosing regimens, are consistent with the notion that reduced activity in the DA system may underlie some of the clinical symptoms of psychostimulant cessation.

\section{EEG BRAIN MAPPING OF DRUG-INDUCED INTOXICATION}

\section{Scott Lukas}

Dr. Lukas described his research using EEG imaging to study the correlates of drug-induced intoxication, and modeling of the EEG dipole source with aid of topographic MRI. EEG mapping of drug intoxication involves consolidating information from many electrode sites and displaying the information in a color-coded format. This format permits the investigator to evaluate topographic alterations in the voltage and frequency components of the ongoing EEG activity. Because of the fine temporal resolution, EEG brain mapping can be performed during specific behavioral tasks performed under the influence of drug. This technique is also useful for analyzing rapidly changing subjective mood states. Measure of drug-induced changes in mood states are measured using an instrumental joystick divided to minimize the artifacts produced on the EEG recording. The results of the EEG mapping show that EEG alpha activity was increased during discrete episodes of euphoria produced by acute administration of cocaine, ethanol, and marijuana. The episodes of euphoria were more frequent during the ascending limb and peak of the plasma ethanol, cocaine and marijuana curves. Interestingly, plasma cocaine levels were dramatically increased if the subject smoked marijuana first. This increase in plasma cocaine levels corresponds to an increase in subjective effects.

Dr. Lukas described advances in localization of the EEG dipole sources which have vastly improved the spatial resolution of EEG. Estimates of dipole origin for event-related potentials (ERPs) during an "oddball" task were calculated via the "inverse" solution, using a 3-shell, 6 parameter model. The origin, magnitude, and orientation of the potential were described using a Cartesian coordinate system, and the location of key electrode sites marked with vitamin E capsules prior to a brain MRI. Coordinates of the dipole were then transposed onto the MR data set. This approach showed sources of spindles and alpha activity appeared most frequently in the thalamus. Increases in signal amplitude increase dipole amplitude, but did not distort the origin of the signal. The combination of EEG and MR imaging procedures capitalize on the strengths of these techniques in temporal and spatial resolution, respectively, and permit direct study of the neurophysiolgical basis of druginduced euphoria in humans. 


\section{DOPAMINERGIC INVOLVEMENT INCOCAINE DEPENDENCE}

\section{John Gatley}

Dr. Gatley and the research group at Brookhaven Laboratories have evaluated the brain dopamine system in cocaine abusers and normal controls using a multiple-tracers approach to image pre- and post-synaptic dopamine neuronal elements, and to measure regional brain glucose metabolism. Because regional brain glucose metabolism is tightly coupled with brain function, this strategy provides a way of linking dopaminergic changes with regional brain function. Fluorine-18-N-methylspiroperidol (NMS) and ${ }^{11} \mathrm{C}$ raclopride were used to assess post-synaptic dopamine $\mathrm{D}_{2}$ receptors, ${ }^{11} \mathrm{C}$ cocaine to measure presynaptic DA terminals and ${ }^{18}$ FDG to measure regional brain glucose metabolism. In addition, ${ }^{11} \mathrm{C}$ raclopride has been used to measure relative changes in DA synaptic concentration secondary to pharmacological stimulation with methylphenidate (MP), a drug that increases synaptic DA by inhibiting the DA transporter. These studies have shown that: 1) uptake of ${ }^{11} \mathrm{C}$ cocaine into basal ganglia is rapid, paralleling the time course of the euphorigenic effects of cocaine; 2) cocaine abusers have long lasting changes in DA post-synaptic receptor availability and in pre-synaptic DA terminals; 3) reductions in DA post-synaptic receptor availability are associated with hypometabolism in prefrontal, cingulate and orbitofrontal regions; 4) cocaine abusers have a blunted response to MP-induced DA changes; and 5) regional brain metabolic changes induced by MP were significantly different in normals than in cocaine abusers. These findings suggest a possible dysregulation of DA receptor function in chronic cocaine users.

\section{SPECT IMAGING OF DA TRANSPORTERS IN COCAINE DEPENDENCE WITH $\left[{ }^{123}\right.$ I $] \beta$-CIT}

\section{Robert Malison}

Dr. Malison presented studies using SPECT neurotransmitter tracers to study pre-and post- synaptic dopamine physiology in primates and in humans. In a paradigm for studying stimulated DA release, systemic injection of methamphetamine into anesthetized baboons produced faster evacuation of the DA post-synaptic radiotracer I-123 IBZM than injections of saline. The increased rate of tracer evacuation in this paradigm may evidence competitive displacement by endogenous DA released by amphetamine. Similar paradigms are now ongoing in humans.

Dr. Malison described the models used to derive estimates of binding potential for radioreceptor ligands, and elegantly demonstrated application of this approach in his recent work measuring the DA reuptake/transporter site in cocaine patients. Early results from within-subjects SPECT imaging of radoactively-labeled $\beta$-CIT, a DA transporter ligand, suggest that transporter sites are higher immediately following cocaine cessation than at a point approximately four weeks later. Transporter sites may be up-regulated during cocaine use in response to the increased levels of synaptic DA. Changes in the DA transporter site during the weeks following the last dose of cocaine may correlate with clinical phenomena of cocaine cessation. 


\section{BRAIN IMAGING DURING DRUG-CRAVING STATES}

\section{Anna Rose Childress}

Dr. Childress reported on the use of cue-induced craving to image the neurochemical and neuroanatomical correlates of drug craving states in cocaine users. Human drug users often experience strong drug desire and arousal when encountering reminders of the drug (drug-buying locations, drug talk, paraphernalia, etc.). Based on this observation, audiotapes and videotapes featuring cocaine-related content were used to evoke craving in the imaging setting. Preclinical studies have shown that cues which signal cocaine activate brain dopamine systems, triggering DA release. These findings suggest the hypothesis that cocaine-related cues may also produce activation of DA systems in humans, and that DA release may be one component of cocaine cue-induced craving.

With regard to neurochemical correlates, Dr. Childress' group has used SPECT brain imaging and the reversibly binding D2 dopamine receptor radioligand, [123I]-IBZM, to measure rate of tracer evacuation in abstinent cocaine patients exposed to either non-drug ("neutral") cues, or to cocainerelated cues (audiotapes, videotapes) which trigger craving. Increased evacuation of this tracer during the cocaine cues, due to competitive binding by endogenous DA, would be consistent with increased DA release to the drug cues. Initial results with IBZM have found similar rates of washout during cocaine-related and neutral cues. The low signal-to-noise ratio of IBZM. and the relative size of the DA signal to cues (smaller and more variable than that occurring to a pharmacologic probe such as amphetamine) make it difficult to optimally test the DA-release hypothesis with IBZM. With regard to neuroanatomical correlates, recent PET studies using $\mathrm{O}$ 15-labeled water as a tracer of regional cerebral blood flow ( $\mathrm{rCBF}$ ) suggest that cocaine users have differential increases in brain blood flow to cocaine cues vs. neutral cues, and that these changes arc often most prominent in limbic regions. Both SPECT and PET studies demonstrated the feasibility of using cue-induced craving in an imaging setting to study the brain correlates of drug craving state(s).

\section{DISCUSSION}

\section{Charles P. O'Brien}

Dr. O'Brien guided the symposium question-and-answer period, and closed the discussion by noting the promise of imaging techniques for understanding of the brain changes which occur during drug use, drug cessation, and drug craving.

\section{REFERENCES:}

Furnished upon request of senior author.

\section{AFFILIATIONS:}

Edythe London, NIDA Addiction Research Center, Baltimore MD

Williams Melega, University of California, Los Angeles, CA 
Scott Lukas, Alcohol and Drug Abuse Research Center, Belmont, MA Anna Rose Childress, University of Pennsylvania, School of Medicine, Philadelphia, PA

Charles P. O'Brien, University of Pennsylvania and Department of Veterans Affairs, Philadelphia, PA

John Gatley, Brookhaven Laboratories, Upton, NY 


\section{DR. FRANKLIN M. ROBINSON MEMORIAL SYMPOSIUM:}

\section{MEDICINAL CHEMISTRY IN DRUG ABUSE RESEARCH}

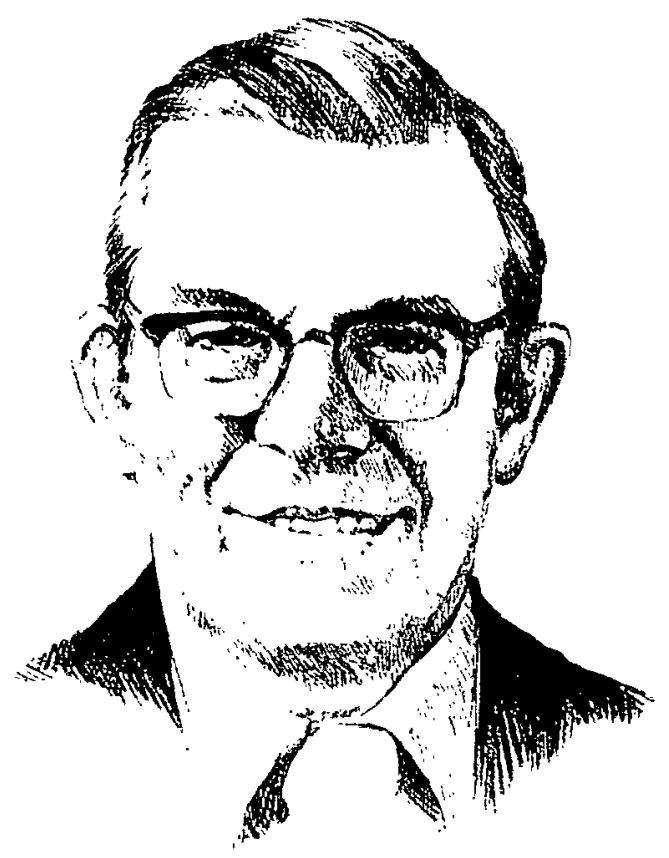

\section{FRANKLIN M. ROBINSON, Ph.D. (1921-1992)}

Born in Arlington, Massachusetts in 1921, Franklin Robinson received a Baccalaureate Degree in chemistry from Worcester Polytechnic Institute in 1943. For the next three years he was a Research Associate at the Sterling-Winthrop Research Institute. During this period, Frank realized that further education would be important for the optimal pursuit of his newly found interest in drug discovery. In 1946 he moved to the University of Minnesota as a chemistry graduate student in the laboratory of Professor C. F. Koelsch. In 1950 he received a Ph.D. degree in organic chemistry from the University of Minnesota and joined the Merck Research Laboratories in Rahway, New Jersey. Frank was a member of the Merck team that discovered mevalonic acid. He also participated in the early work on vitamin B12 and Coenzyme Q. 
After serving a term as Chief of Staff for the executive Director of Basic Research, Frank moved to the Merck Research Laboratories in West Point, Pennsylvania. Here he began his studies on analgesic agents. These studies, which were focused on morphinans and benzomorphans, produced MK-901 as a development candidate. His laboratory was the first to report the synthesis of hexahydroindeno[2.1b]pyrroles as a novel benzomorphan-related template for construction of potent analgesic agents. During this period Dr. Robinson began to play a major role in the Merck Recruiting Program. His skill in identifying and attracting top talent to Merck is legendary. Among his successes, Frank could count two recruits who eventually became Vice-Presidents and more than half a dozen who rose to the rank of Senior Director. Perhaps more importantly he was an able and remarkably effective mentor for several generations of Merck scientists. His enthusiasm for science was infectious. He encouraged young scientists in the rigorous pursuit of new ideas and set, by example, a high standard for performance. Although Frank completed his career at Merck more than five years ago, the positive impact of his leadership is still very much in evidence today. The scientists that he recruited and those that he mentored continue to emulate the spirit of cooperation, enthusiasm for science and commitment to excellence that he taught and encouraged. This special chemistry symposium of the 56th Annual Scientific Meeting of CPDD is respectfully dedicated to Dr. Franklin M. Robinson.

\section{AFFILIATION}

Paul S. Anderson, Ph.D., Vice President, Chemistry and Physical Sciences Research and Development, DuPont Merck Pharmaceutical Co., Wilmington, DE 19880-0353. 


\title{
FRANKLIN ROBINSON MEMORIAL SYMPOSIUM ON MEDICINAL CHEMISTRY IN DRUG A B USE RESEARCH
}

\author{
A. H. Newman; P. S. Portoghese; S. Calderon; J. Lewis; R. H. \\ Kline; P. Anderson; and K. C. Rice
}

\section{INTERACTION OF SELECTIVE NONPEPTIDE LICANDS WITH OPIOID RECEPTORS}

\section{Philip S. Portoghese}

The opioid receptor antagonists, naltrindole (NTI) and norbinaltorphimine (norBNI), contain key moieties that enhance binding to $\delta$ and $\kappa$ receptors, respectively. Structure-activity studies have suggested that the indolic benzene moiety of NTI and the $\mathrm{N}^{2}$ basic nitrogen of norBNI are responsible for the selectivity of these ligands. A simple model based on the "message-address" concept of Schwyzer (Ann. N.Y. Acad. Sci. 1977, 297, 3) has been employed to rationalize the selectivity. In this connection, the indolic benzene moiety of NTI was considered to mimic the Phe $\mathrm{P}^{4}$ phenyl group of enkephalin, and the $\mathrm{N}^{2}$ basic group of norBNI was proposed to mimic $\mathrm{Arg}^{7}$ of dynorphin. That is, the nonpeptide moieties were thought to serve an address function in a manner similar to the corresponding groups in the opioid peptides.

Structure-activity studies of NTI congeners have revealed that ligands whose aromatic groups share the same conformational space with the indolic benzene moiety of NTI, possess higher $\delta$ antagonist potency than other analogs in the series, suggesting that access to an aromatic binding subsite is important for $\delta$ antagonist activity. However, the fact that NTI cannot be converted to a full agonist by replacement of its cyclopropylmethy 1 with a methyl group (oxymorphindole, OMI) suggests that $\delta$ agonists and $\delta$ antagonists have different conformational requirements. In this connection, molecular dynamics studies of $\left[\mathrm{D}-\mathrm{Ala}^{2}, \mathrm{D}-\mathrm{Leu}\right]$ enkephalin have revealed that the Phe ${ }^{4}$ phenyl group in most of the low energy conformers is roughly perpendicular to the indolic benzene moiety of OMI. These studies have led to the synthesis of 7-spiroindanyloxymorphone (SIOM), a ligand whose benzene moiety is perpendicular to ring $\mathrm{C}$ of the opiate. The finding that SIOM is a full agonist at $\delta$ receptors, both in vitro and in vivo, is consistent with the idea of different recognition sites for $\delta$ agonists and $\delta$ antagonists. The fact that mutation of Asp 128 to Asn in the $\delta$ receptor causes the loss of agonist but not antagonist binding (Kong, et al., J. Biol. Chem. 1993, $268,23055)$ also supports this conclusion.

A similar situation appears likely with norBNI in that its $\mathrm{N}^{2}$ basic group does not interact with the same subsite on the $\kappa$ receptor as the basic group in the address segment of dynorphin. The fact that the $\mu$ opioid receptor has been proposed to possess separate recognition sites for agonists and antagonists (Portoghese and Takemori, J. Med Chem. 1983, 26, 1341) also suggests that this is a general feature in the opioid class of G-protein coupled receptors.

In conclusion, the idea of discrete message and address subsites on opioid receptors has been useful in the design of selective opioid antagonists. However, the concept should not be taken to mean that the message and address subsites of 
agonists and antagonists are identical because of the possible different modes of interaction of agonist and antagonist ligands with opioid receptors.

\section{DEVELOPMENT OF SELECTIVE NONPEPTIDE LIGANDS FOR FURTHER CHARACTERIZATION OF DELTA-OPIOID RECEPTORS.}

\section{Silvia Calderon}

The synthesis of nonpeptide agonists and antagonists with high selectivity for delta $(\delta)$ opioid receptor subtypes is an important and challenging problem in medicinal chemistry. Nonpeptide ligands have the advantage that they are less subject to metabolism than peptides and also can penetrate the blood-brain barrier. Recently, a novel nonpeptide $\delta$-opioid receptor ligand, $( \pm)$-BW373U86, $[( \pm)-1]$, was reported and appears to be a prime template for the discovery of new probes for the $\delta$ receptor. All the pharmacological studies of this compound to date were performed using the racemate. To best utilize this important lead, studies with the optically pure enantiomers of $( \pm)-\mathbf{1}$ are required, since often drug enantiomers have different and in some cases opposite pharmacological effects.

We synthesized the optically pure enantiomers of $( \pm)$-BW373U86, their benzylic epimers and the corresponding methyl ethers from the enantiomers of 1-allyltrans-2,5-dimethylpiperazine. Receptor binding studies in rat and in mouse brain showed that the phenolic enantiomers of BW373U86 and their benzylic epimers have high affinity for $\delta$ and $\mu$ receptors. Two of the methyl ether isomers of BW3737U86, (+)-2 and (-)-3, immediate precursors in our synthetic route, have shown the most striking results. These compounds exhibit a dramatic increase in selectivity for $\delta$ receptors over $\mu$ receptors by approximately 2000 -fold in opioid binding assays. Thus, methylation of the phenolic group virtually eliminates the affinity of these compounds for $\mu$ receptors.

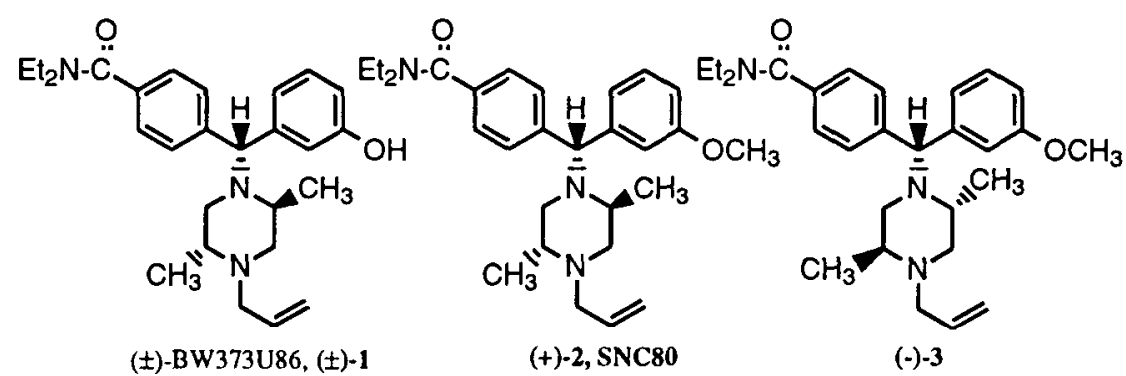

Studies with these compounds in the isolated mouse vas deferens (MVD) and guinea pig ileum (GPI) bioassays revealed that the $\delta / \mu$ selectivity of $(+)-2$ was about 2000-fold as in the binding assays but the selectivity of (-)-3 was only 49fold. At $1 \mu \mathrm{M}$, the $\delta$ receptor antagonist ICI174,864, but not the $\mu$ receptor antagonist CTAP, showed potent antagonism of the effects of (+)-2 in the MVD bioassay. These data collectively show that $(+)-2$ is a potent, $\delta$ receptor agonist and suggest that this compound will be a valuable tool for further study of the $\delta$ opioid receptor structure and function including its role in addictive diseases. 


\section{OPIOIDS - FROM ANALGESICS TO TREATMENT DRUGS}

\section{John Lewis}

Drug discovery programs in the opioid field have in the past been directed to "non-addictive" analgesics. The recognition of" multiple opioid receptors turned attention away from $\mu$ towards $\delta$ and particularly $\kappa$. $\kappa$-agonist analgesics do not have significant abuse potential but despite substantial research investment by the pharmaceutical industry in the search for specifick-agonists, it is unlikely that such analgesics will contribute significantly to pain management in the future. The prospects for specific $\delta$ agonists do not appear much brighter.

For the medicinal chemist involved in drug discovery, opportunities to design opioids as pharmacotherapies for opiate abuse have become of increasing importance and have renewed interest in activity at $\mu$ receptors. Agonist (methadone, LAAM) and antagonist (Naltrexone) maintenance treatments are available and a partial agonist (buprenorphine) is in late development. The latter may be of particular value in the treatment of dual opiate/cocaine abuse. Alternatives and improvements are required to provide appropriate treatment for addicts in different stages of the addiction cycle.

We have been attracted to the concept of $\mu$ partial agonists of varying intrinsic activities to provide a range of treatments to cover the spectrum between the full agonists and the pure antagonist. An important lead was provided by methoclocinnamox (MC-CAM), a long lasting $\mu$ partial agonist which is converted to the irreversible antagonist clocinnamox (C-CAM) by metabolism. We have synthesized and had evaluated by Jim Woods a number of alternative ethers of C-CAM. In comparison with the methyl ether (MC-CAM) the alkyl, propargyl and cyanomethyl ethers have more pronounced agonist actions; the CPM ether by contrast appears to be more antagonist that MC-CAM and the npropyl ether quite similar to MC-CAM. In binding assays the ethers are remarkable in displaying affinities for $\mu$ receptors equal to or in the case of the propargyl ether, significantly higher than that of the parent phenol C-CAM. They also show selectivity differences between ethers and in comparison to the phenol which has significantly higher $\boldsymbol{K}$ and $\delta$ affinities than the ethers.

As potential pharmacotherapies for opiate abuse, MC-CAM and the other analogs offer an agonist, reinforcing response followed by prolonged receptor blockade as the antagonist C-CAM is produced by metabolism. Since it may not be desirable to block opioid receptors for such a long period, we have evaluated some close analogs of MC-CAM and C-CAM in which the Michael acceptor cinnamoylamido group which could form a covalent bond to the receptors has been removed by hydrogenation and then by reduction of the amide function. It was expected that these changes would remove irreversible antagonist actions from the pharmacological profiles. However, both morphinones showed the character of non-competitive $\mu$ antagonists of exceptional in vitro potency. This suggests that the irreversible antagonism of C-CAM may be due in part to a noncovalent binding interaction probably involving the lipophilic chlorophenyl group. 


\section{SUBSTITUTED 3-CARBAMOYLOXYECGONINE METHYL ESTER ANALOGS: NOVEL PROBES FOR THE COCAINE RECOGNITION SITE ON THE CENTRAL DOPAMINE TRANSPORTER}

\section{Richard H. Kline}

In the search for compounds to further characterize the cocaine recognition site on the central dopamine transporter several 3-( $N^{\prime}$-phenylcarbamoyloxy)ecgonine methyl ester analogs were prepared. The synthesis of these compounds started with the conversion of $(1 R)$ cocaine to $(1 R)$ ecgonine methyl ester via hydrolysis in dilute $\mathrm{HCl}$ and subsequent re-esterification with $\mathrm{CH}_{3} \mathrm{OH} / \mathrm{H}_{2} \mathrm{SO}_{4}$. (1R)Ecgonine methyl ester was then reacted with appropriately substituted phenyl isocyanates yielding the $(1 R)$ stereoisomers of the desired analogs in good yields. These compounds were tested for their ability to inhibit $\left[{ }^{3} \mathrm{H}\right]$ cocaine binding to rat striatal tissue and $\left[{ }^{3} \mathrm{H}\right]$ dopamine uptake into synaptosomes prepared from the same tissue. The most potent of these analogs was $(1 R-2-$ exo-3-exo $)-3-\left(N^{\prime}-3\right.$ 'nitrophenylcarbamoyloxy)-8-methyl-8-azabicyclo[3.2.1]octane-2-carboxylic acid methyl ester. $\mathrm{IC}_{50}$ values for inhibition of binding and uptake were 37 and 178 $\mathrm{nM}$, respectively. Generally, compounds which contained meta substituents on the phenyl ring of the $\mathrm{C}_{3}$ phenylcarbamoyloxy moiety were more potent than those with para substituents. Also, analogs which contained electron withdrawing substituents on the mentioned phenyl ring had considerably higher potencies than those with electron donating substituents. A further objective of this research was to design and synthesize ligands which could be used for the eventual purification of the central dopamine transporter. Thus, analogs which could potentially interact irreversibly with the cocaine binding site containing isothiocyanato or azido moieties were synthesized from appropriately substituted amino compounds. Preincubation of synaptosomal membranes with the meta- and paraisothiocyanato analogs, followed by two washes, resulted in inhibition of $70 \%$ and $85 \%$ of $\left[{ }^{3} \mathrm{H}\right]$ cocaine binding. After pre-incubation in reduced lighting, metaand para-azido compounds inhibited $0 \%$ and $13 \%$ of $\left[{ }^{3} \mathrm{H}\right]$ cocaine binding, while following pre-incubation under ultraviolet light the inhibition increased to $61 \%$ and $68 \%$, respectively. Thus, the isothiocyanato derivatives appear to bind covalently to the cocaine binding site, whereas the azido derivatives are apparently photoaffinity labels at the same site. Either type of analog may be useful in the purification of the cocaine recognition site on the central dopamine transporter.

\section{STRUCTURE-ACTIVITY-STUDIES WITH ANALOGS OF MK 801}

\section{P. S. Anderson}

The discovery of dizocilpine, MK-801 (I, 5S,10R), began with an attempt to optimize the modest antiseizure and apparent anxiolytic activities exhibited by a compound that had been selected for this type of screening because of its novel structure. Pursuit of this objective by use of established pharmacological assay methods which employed intact animals led to the discovery of 9,10,11-trimethyl9,10-dihydroanthracen-9,10 -imine (II). Compound II proved to be a potent antiseizure agent with central sympathomimetic activity, thus suggesting potential for development as a nonsedating drug to treat epilepsy. This course of action was precluded by the observation that compound II was susceptible to oxidative deamination to afford 9,10-dimethylanthracene, a potent mutagen. 
The structure of compound II suggested a strategy for circumventing this problem. Its carbon skeleton was reorganized by moving one of the identical bridgehead methyl groups into the central ring of the hydrocarbon framework as a rnethylene unit. Through this maneuver, structures such as that of compound I were obtained. The topography of compound I is very similar to that of compound II; however, the additional methylene unit blocks the cheleotropic elimination pathway, and thus it is stable toward deamination under oxidative conditions. Unlike compound II, compound $\mathbf{I}$ is a chiral molecule. The enantiomer with the 5S, 10R configuration as its hydrogen maleate salt is dizocilpine (MK-801). As was the case with compound II, dizocilpine proved to be a potent antiseizure agent with significant central sympathomimetic activity.

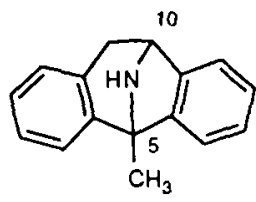

1

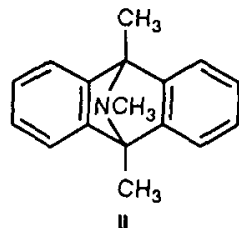

॥

Because a mechanism to account for dizocilpine's pharmacological actions had not been established, studies were initiated to address this important issue. $\left[{ }^{3} \mathrm{H}\right]$ Dizocilpine was synthesized and used to develop a radioligand displacement assay and to map binding sites in brain. The binding site distribution in rat brain was comparable to that previously observed for glutamate. This observation and results subsequently obtained from electrophysiological studies on brain slices were in accord with selective, noncompetitive blockage of the N-methyl-Daspartate (NMDA) subset of glutamate receptors as the mechanism for the pharmacological activity of MK-801. Agents of this type have potential in management of seizure disorders, as well as in management of cerebral ischemia associated with stroke and/or cardiac arrest.

\section{NOVEL 3 $\alpha$-DIPHENYLMETHOXYTROPANE ANALOGS ARE POTENT DOPAMINE UPTAKE INHIBITORS WITHOUT COCAINE-LIKE BEHAVIORAL PROFILES.}

\section{Amy Hauck Newman}

Novel dopamine uptake inhibitors have proven to be useful for elucidation of the role of the dopamine transporter in the pharmacology of cocaine. Benztropine (BZT, $3 \alpha$-diphenylmethoxy)-1 $\alpha \mathrm{H}, 5 \alpha \mathrm{H}$-tropane) is a dopamine uptake inhibitor that is equipotent to cocaine and exhibits CNS stimulant activity in animal models. BZT shares the common tropane ring moiety of cocaine and the diphenylmetboxy substituent of another series of selective dopamine uptake inhibitors i.e. GBR 12909. Hence, BZT appeared to be an interesting template for structural modification towards the development of novel probes of the dopamine transporter. An initial series of puru-substituted BZT analogs was prepared generally by reacting tropine or pseudotropine with the appropriate parasubstituted benzhydrylchloride at $160{ }^{\circ} \mathrm{C}$ and isolating the products as the $\mathrm{HCl}$ salts. All of these compounds monophasically displaced $\left[{ }^{3} \mathrm{H}\right]$ WIN 35, 428 binding in rat caudate-putamen with $\mathrm{K}_{\mathrm{i}}$ values ranging from 11.8 to $2000 \mathrm{nM}$. 
The rank order of potency for both binding and inhibition of $\left[{ }^{3} \mathrm{H}\right]$ dopamine uptake was 4',4"diF $>4^{\prime}, 4^{\prime \prime} \mathrm{diC} 1>4^{\prime} \mathrm{C} 1>4^{\prime} \mathrm{OMe}>\mathrm{BZT}>3 \quad \beta-44^{\prime} \mathrm{Cl}>4^{\prime}, 4^{\prime \prime} \mathrm{diOMe}$. Interestingly, when the diphenylmethoxy substituent was in the 3-position, as is the $3 \beta$-benzoyl group of cocaine, potencies for binding and inhibition of $\left[{ }^{3} \mathrm{H}\right]$ dopamine uptake were decreased by approximately 30 -fold. The lack of a $2-$ position substituent further distinguished this series from the potent cocainerelated analogs. Additionally, phenyl ring substitution resulted in large differences in binding potency. Behaviorally, these compounds demonstrated low efficacy locomotor stimulation in mice as compared to cocaine, despite their (generally) higher binding potency. Likewise, rats trained to discriminate cocaine from saline did not recognize these analogs as being cocaine-like at behaviorally active doses. These compounds are the first tropane analogs to be reported that have high affinity for the dopamine transporter, block dopamine uptake but do not exhibit a cocaine-like behavioral profile. Drug interaction studies are underway to determine whether these compounds may act as functional cocaine antagonists.

\section{AFFILIATIONS:}

Department of Medicinal Chemistry, University of Minnesota, Minneapolis, MN Laboratory of Medicinal Chemistry, NIH/NIDDK, Bethesda, MD 20892

School of Chemistry, University of Bristol, England

Psychobiology Section, NIH/NIDA, Baltimore, MD 21224

Merck Sharp \& Dohme Research Laboratories, West Point, PA 19486 


\title{
E F F E C T S OF AMOUNT OF SERVICES ON OUTCOMES DURING TREATMENT: PRELIMINARY ANALYSIS FROM TWO NATIONAL STUDIES OF COMMUNITY-BASED TREATMENT PROGRAMS
}

\author{
R. L. Hubbard, R. M. Etheridge, S. G. Craddock, and \\ G. Dunteman
}

It has been generally demonstrated that longer time spent in drug abuse treatment is related to more positive outcomes. In an environment of costcointainment, a critical question is whether a sufficient and equally effective dose of treatment can be delivered in a shorter time frame. Data from two national studies of treatment effectives, TOPS (1979-81) and DATOS (199193) address the issue of measuring, analyzing and interpreting effects of treatment dose. Preliminary investigation focused on (1) the nature and extent of counseling and services provided during the initial three months of treatment; (2) the cognitive and behavioral changes occurring during three months of treatment; and (3) the potential effect of this treatment dose in interaction with moderator and mediating variables. The initial analysis was restricted to a sample of methadone (TOPS[n]-2,185; DATOS[n]-908), and outpatient (TOPS[n]-763, DATOS[s]-855) clients.

The amount and diversity of counseling and services reported between 1980 and 1992 has declined substantially. Despite increases in self-reported needs for services in many areas (medical, family, etc.), clients in 1991-93, compared to $1979-81$, reported receiving fewer services, more unmet needs for medical services $(39.6 \%$ versus $17.8 \%$ in methadone, and $32.0 \%$ versus $9.2 \%$ in outpatient), family services $(52.8 \%$ versus $17.0 \%$ in methadone and $63.0 \%$ versus $8.1 \%$ in outpatient) and other services including psychological and vocational.

The amount of counseling or services do not appear to have a direct main effect on drug use outcomes during methadone and outpatient treatment. None of the preliminary measures of treatment dose, including days attended, total hours of counseling, number of services, or number of met needs, had a significant positive effect. The clients using cocaine during treatment were more likely to report receiving more services. The effect of treatment dose may be moderated by the behavior and cognitions of clients prior to admission. The numbers of problems and higher levels of problem indicators prior to treatment was predictive of higher rates of use during treatment.

Involvement with the criminal justice system had significant, but opposite effects on cocaine use in methadone and outpatient clients probably due to the formal criminal justice referral of outpatient clients. The effect of treatment dose may be mediated by cognitive changes occurring during drug treatment. Cognitive measures such as relationships with a counselor, perceived hepfulness of treatment, and self-efficacy were positively and significantly related to reduced use during treatment. These results suggest the need for a more comprehensive, complex, multivariate framework to investigate the effect of treatment dose. This framework needs to consider contextual effects of program and system, moderating influence of client characteristics, mediating 
effects of cognitive change, as well as the appropriate method of assessing and describing treated dose.

\title{
ACKNOWLEDGEMENT
}

Supported by NIDA Contract No. 271-89-8233 and Grant No. 1 P50 DA 06990-01A1

\section{AFFILIATION}

Substance Abuse Treatment Research Program, Research Triangle Institute, Research Triangle Park, North Carolina

\section{PSYCHOSOCIAL TREATMENTS FOR COCAINE ABUSE: TREATMENT INTENSITY AS A PREDICTOR OF LONG-TERM CLINICAL OUTCOMES}

\author{
J. A. Hoffman, B. D. Caudill, J. J. Koman, III, J. W. Luckey, \\ P. M. Flynn, and D. W. Mayo
}

This report summarizes outpatient treatment outcome results at 12-months post-treatment for 184 cocaine-abusing clients, 96\% primarily crack smokers, from a study of six psychosocial treatment interventions which varied in level of intensity. The treatments included group counseling two or five times a week either alone or adding individual therapy alone or with family therapy. Subjects were 60\% male, 95\% African American, average age 31, 20\% married or living with a partner, 39\% involved in criminal justice system, 39\% employed full-time in year prior to treatment, and on average, had a 12 th grade education. The response rate was $66 \%$ of clients eligible for follow-up, $38 \%$ of whom completed the 4-month treatment program. Across all groups, the sample went from $78 \%$ self-reported regular crack use (weekly or greater) in the year prior to treatment to $19 \%$ weekly users during the year following treatment. Although there were no significant differences in the reduction of crack use between groups, the number of days in treatment and the number of treatment sessions attended were significantly related to reductions in crack use. As a group, post-treatment regular users spent an average of 33.8 days in treatment vs. 59.5 days for clients who were not regular users $(\mathrm{p}<.005)$; and post-treatment regular users attended an average of 12.4 sessions vs. 24.5 sessions for clients who were not regular users $(\mathrm{p}<.008)$. Reductions were also found in the percentage of clients reporting involvement in illegal activities for monetary gain during the 12 month period following treatment (from 36\% in the year prior to treatment to $9 \%$ in the year after) which, when grouped by the presence or absence of illegal behavior, was also significantly related to days in treatment $(\mathrm{p}<.03)$.

Post-treatment full-time employment status decreased slightly from the intake report of employment. The results indicate that longer participation in outpatient treatment and treatment exposure were both significantly related to reductions in regular crack use. Once an optimal level of outpatient treatment is offered, emphasis may be placed on interventions and incentives to enhance motivation and participation and to provide specific additional services. 


\section{ACKNOWLEDGEMENT}

Supported by NIDA Grant No. 5R18 DA06 168.

\section{AFFILIATIONS:}

Center for Drug Treatment and Research, The Koba Institute, Inc., 1156 Fifteenth St. NW, Suite 200, Washington, DC 20005

Center for Social Research and Policy Analysis, Research Triangle Institute, 3040 Cornwallis Road, Research Triangle Park, NC 27709

\section{DOSE-RESPONSE STUDIES OF PSYCHOSOCIAL SERVICES DURING SUBSTANCE ABUSE TREATMENT}

\section{A. T. McLellan}

Is it possible and practical to increase treatment effectiveness by "matching" different "types" of patients to particular kinds of programs or treatments? What are the system barriers to "matching"? Data is presented from a prospective study of patient-treatment matching involving two treatment settings and four accredited, private programs treating 460 employed alcohol and cocaine dependent patients referred from a large EAP.

Stage I - patients were evaluated by the EAP using a standard clinical interview and referred in the normal manner to one of the tour programs. All patients were followed weekly by independent researchers monitoring the nature and number of the services received. Patients were re-contacted (94 percent) six months after treatment discharge and re-evaluated. These data were used to develop a model of patient-program and treatment service matching for the second stage of the study.

Stage II - using the stage one decision tree to randomly assign 387 new patients to: "matched" programs and service profiles. "Matched" patients were compared against controls treated at the same programs in the standard manner but not given the matched services. Outcome measures were drug and alcohol use, empoyment, crime, use of medical and social services.

Results and Conclusions - It was impossible to actually match patients to the programs due to insurance constraints, managed care, geography and patient resistance. However, matching treatment services to patient problems at the program level was quite successful, "Matched" patients received the same total services as control patients, but were less likely to drop out early. Six-month outcome comparisons showed that matched patients had 15-25 percent better outcomes than control patients treated in the same programs by the same staff under standard conditions. Problem-service matching at the program level was easy to implement, well accepted by clinical and administrative staff and cost an additional three percent (of program budgets) to institute.

\section{AFFILIATIONS}

Pennsylvania Veterans Administration Treatment Evaluation Center, Philadelphia Veterans Administration Medical Center, University Avenue, Philadelphia, PA 19104 


\title{
ETIOLOGY, EPIDEMIOLOGY AND NATURAL HISTORY OF HEROIN ADDICTION: A SOCIAL SCIENCE APPROACH
}

\author{
J. C. Ball, D. N. Nurco, R. R. Clayton, M. Lerner, T. A. Hagan, \\ and G. A. Groves
}

Drug addiction can be explained as a biomedical, psychological, or sociological phenomenon. In this symposium, the overall explanatory power of a social science approach was delineated and some current research findings were presented. It was held that this approach provides a more comprehensive explanation of the problem of drug abuse in the United States than other approaches. Although sociological findings were emphasized, it was recognized that other social science disciplines (e.g., anthropology, social psychology and economics) also make significant contributions.

A social science approach to the study of drug dependence involves consideration of fundamental issues related to the nature, scope and dynamics of this social problem. Thus, this approach addresses such basic issues as: (1) the history of drug abuse patterns and the changing populations of users; (2) recognition that specific drug abuse patterns are culturally determined--that cultures (and subcultures) differ in the availability of drugs and the extent of abuse; (3) that the demographic (and epidemiological) characteristics of abusers depend upon the time period, nation and locale selected for study; (4) the necessity to delineate the specific drug (or drugs) of abuse, route of administration and length of dependence; (5) the etiology or social context in which drug abuse begins; (6) the influence of major institutions (e.g., family, community, peer group, schools, media) upon the onset and continuation of drug dependency; (7) why drug abuse is more prevalent in certain populations than others; (8) determination of institutional supports which promote successful treatment and rehabilitation, including consideration of how persistent behavior in subcultures can be changed.

With respect to historical and cultural contexts, it was noted that demographic populations at high risk for opiate addiction have differed by era and location within the United States. Similarly, for those who do become addicts, age at onset of daily opiate use and other characteristics of their addiction careers also differ by period and place of residence. Furthermore, it is significant that comparable or even greater variations in addiction characteristics and consequences have been found in other nations Thus, the problem of opiate addiction differs by nation and historical period, although most physiological and pharmacological aspects of addiction, such as physical dependence, remain constant.

Consequently, social science studies of drug addiction have usually focused on particular populations of abusers, classified specific drugs of abuse, and formulated definite scientific questions to investigate. Therefore, it is necessary to indicate which population (adult males, metropolitan slum dwellers, teenage females, college students, army personnel, factory workers, prostitutes, criminals, doctors, pregnant housewives, etc.) and which drugs of abuse (heroin, morphine, PCP, cocaine, marijuana, barbiturates, etc.) are to be studied. In 
addition, it is important to measure frequency of use as well as to note route of administration.

With respect to the etiology of drug dependence in the United States, the role of peer group and community are of paramount importance, especially with respect to persistent intravenous drug use. Thus, most heroin dependency starts as part of a peer group recreational activity. This onset behavior is similar to juvenile delinquency in that the boys are seeking status in the peer group by taking part in a search for kicks and mutual excitement. Initiation into drug use is voluntary rather than accidental or coercive. The incipient addict tasks to join the drugtaking group whose members arc seen as friends. (The onset pattern for females is different; most are initiated by their boyfriends or other males).

The family also has a crucial role to play in the prevention of drug abuse among children. However, many children do not have responsible parents and, consequently, they are deprived of suitable early socialization. In this regard, there are not only orphans and unwanted children, but parents who are themselves opiate addicts, criminals, or prostitutes.

In a very real sense, addiction is a community problem, rather than merely an individual problem. This is because addiction is maintained and spread by drugusing cohorts from generation to generation in metropolitan slum areas. Furthermore, the fact that minority group members constitute a major portion of inner-city dwellers only exacerbates the problem of awakening public interest and support. So the scope and complexity of the slum problem remain intact, and the poorer areas of our cities continue to be ignored.

When attention is turned to the rehabilitation of persistent drug abusers, it seems apparent that treatment alone cannot be expected to contain the problem. This is because no one approach or single institution will be sufficient to meet the heroin addiction problem in the United States. Rather, a coordinated societal approach is necessary, in which increased resources will be organized to meet prevention, education, and treatment needs of communities, occupations, and other populations. In order to explicate further the social science approach, attention was focused on several of those basic issues.

\section{EPIDEMIOLOGY IN THE UNITED STATES}

Epidemics can occur if there is an increase in the percentage of the population using drugs, in the number of heavy users, and/or there is an increase in the amount of drugs consumed by both those in the general population and the heavy users. In order to adequately understand the genesis, course of development and regression, and the correlates and causes of a drug epidemic, there is one requisite: valid and reliable data. In the U. S., three major sources of national data about the extent of drug abuse are: (1) Monitoring the Future Data about high school seniors; (2) DAWN data from hospital emergency admissions; and (3) National Household Surveys of the general population.

The population of the United States, 12 years old and older, is clearly drug experienced. Data from the 1992 National Household Survey show that $33 \%$ (67.5 million) people have used marijuana, 4.4\% (8.9 million) in the prior month. Some 22.6 million (11\%) have used cocaine, 1.3 million $(0.6 \%)$ in the past month. Some 98.4 million (48\%) report past month use of alcoholic beverages while 53.9 million $(26.2 \%)$ report past month of smokeless tobacco. 
The DAWN system has been in existence for a considerable period of time. The emergency room episodes for cocaine, heroin, and marijuana were examined in six-month increments from January-June 1988 through January-June 1992. These data show a drop until the period July-December 1990, followed by a steady increase for all three drugs with the latest period being 1.56 times as high as the trough for cocaine, 1.94 times as high as the trough for heroin, and 2.11 times as high as the trough for marijuana.

The Monitoring the Future data on high school seniors show a rather dramatic decline in drug use, since 1979 for marijuana and since 1982 for cocaine. However, comparing 1992 to 1993 data, there were statistically significant increases in use of marijuana (8th, 10th, 12th grade). and daily use of cigarettes in the past month (8th, 10th, 12th grade).

The following conclusions were drawn from the Monitoring the Future study. These changes are real and may constitute a "wake-up" call for the American public. There should be genuine concern that the reversal of the downward trend in drug use could signal the emergence of a new epidemic of drug use. On a number of occasions during the past 20 years there have been assertions by highly placed officials that we have "turned the proverbial corner" on drug abuse. Each time, the assertion has not only proven to be wrong, but has seemed to delay timely response to the changes. It would be tragic if nothing were learned from this history of bad guesses about where the trend line is going.

\section{WOMEN'S ISSUES}

There is little empirical information on differing treatment needs of women in general, or subgroups of women (e.g., opiate dependent, cocaine dependent, Latin or African-American women). Despite this paucity of data, a number of small studies coupled with clinically relevant information have begun to provide a basis for further investigation into appropriate services for women. In addition, the information and data raise important questions for treatment provision, research, and policy planning.

Women bring a number of critical issues to treatment settings that require comprehensive services. These issues include but are not limited to early incest and drug use; physical abuse; family or origin chemical abuse; educational deficits; sporadic employment in low paying jobs; lack of transportation and child care; safe housing problems; violent current relationships; and inconsistent parenting skills. Such diverse and multiple difficulties can precipitate problems and symptoms ranging from: (1) psychosis; (2) flashbacks; (3) depression and anxiety; (4) present behaviors that appear to be associated with possible developmental delays at age of first use; (5) an inability to participate in vocational/educational programs due to poor coping and developmental skills; (6) difficulty in accessing treatment because of a lack of transportation and child care; and (7) repeated negative behaviors and coping mechanisms in current relationships.

Research should address: (1) the development of practices that encourage sensitivity to individual treatment progression, rather than universal measures; (2) broader definitions of treatment success; and (3) methdologies that control for factors that can impede reliable outcome measures; (e.g., lack of housing, transportation, and child care; bias against treatment modalities such as 
methadone; and behaviors associated with arrested developmental levels).

\section{FAMILIES OF NARCOTIC ADDICTS}

The importance of family factors of males respondents aged 12-14 as predictors of narcotic addiction, highlight especially the role of the father in this regard, especially the natural father family, The degree of attachment at ages 12-14 to onee's parents, especially to one' father, is a major determinant of how strong a young person's resistance will be to the temptations offered by addiction in neighborhoods where high levels of drug abuse are prevalent.

A positive home atmosphere and a strong parental commitment is similarly a strong predictor of the addiction history of young people aged 12-14. Further analyses of data, to be carried out shortly, will permit estimation of the percent of variance in addiction or non-addiction explained by these factors, especially after controlling for the effects of neighborhoods, the character of respondents' peer groups, deviance within the family prior to the respondents' age 11, and others.

Studies reported here have thus far focused on epidemiology, but they need to be taken one step farther with the creation of classes of patients that have utility for a clinician. For example, approximately $40 \%$ of the population studied came from families without two natural parents in the household and the addict subject perceived of his father in a negative fashion. By proceeding to identify other variables and in the process create identifiable types, clinicians can see and develop specifically tailored techniques in order to change behavior.

\section{CHARACTERISTICS OF METHADONE MAINTENANCE PATIENTS}

With regard to drug abuse patients in treatment in the U. S., the characteristics of 120,000 opiate addict patients in 650 methadone maintenance programs in the U. S. were delineated. The marked metropolitan concentration of this patient population was noted (e.g., one-third reside in New York City), was its overrepresentation of males and minority group members. It was found that onset of heroin addiction for males commonly commenced as a peer-group recreational activity at an early age (14-18 years); for females onset commonly was begun with their addicted boyfriends. Most patients of both sexes had five or more years of intravenous drug abuse prior to their present treatment and most also had lengthy criminal careers. The general effectiveness of methadone maintenance treatment in reducing IV drug use and criminal behavior was discussed.

\section{CONCLUSION}

From the social science perspective, then, solution to the drug abuse problem does not lie in simply deriving chemical agents to be used in the control of addicts' emotions and behavior or in the mechanistic application of technical innovations. Solutions that do not take into account the basic motivations and propensities underlying addictive behavior are destined to failure. Addicts, for example, can be remarkably creative in achieving their own ends; those working with them must understand this and use laboratory advances as supplements in 
dealing with more global aspects of the individual. Unfortunately, there is no "magic bullet" for the containment of drug deviancy.

\section{AFFILIATIONS}

University of Kentucky, Lexington, Kentucky University of Maryland, Baltimore, Maryland Johns Hopkins University, Baltimore, Maryland University of Pennsylvania, Philadelphia, Pennsylvania Addiction Research Center, Baltimore, Maryland 


\title{
CLINICAL PHARMACOLOGY OF BUPRENORPHINE
}

\author{
M. L. Stitzer, S. L. Walsh, E. C. Strain, K. J. Schuh and G. E. \\ Bigelow
}

The clinical pharmacology profile of buprenorphine derived from laboratory research is generally consistent with the known properties of a mu partial agonist and supports the potential utility of buprenorphine for the treatment of opioid dependence.

Mu agonist profile: Buprenorphine constricts pupils, produces opioid-like subjective effects and analgesia.

Dose-effect ceiling: Human subjects have been tested with acute doses up to $32 \mathrm{mg} \mathrm{s} .1$. Pupil constriction, respiratory depression and subjective reports have all shown maximal effects at doses well below $32 \mathrm{mg}$ (2-16 mg buprenorphine depending on the specific measure). The ceiling on respiratory depression means that this is a very safe medication. Examination of plasma drug levels showed a linear increase with dose, suggesting that the dose-effect ceiling was not due to absorption limitations of the sublingual administration route.

Duration of action: Buprenorphine has a long duration of action. Pupil constriction was apparent for 48 hours after acute s.1. doses of $2 \mathrm{mg}$ and higher. Plasma levels were elevated for $48 \mathrm{hrs}$ after $16 \mathrm{mg}$ and $96 \mathrm{hrs}$ after 32 $\mathrm{mg}$ buprenorphine. The long plasma half life of high doses supports the possibility of dosing on a less than daily basis in treatment.

Blockade of opioid agonists: Buprenorphine attenuates effects of shortacting opioids given as a challenge during chronic treatment. Extent of attenuation was directly related to the maintenance dose from $2-16 \mathrm{mg} / \mathrm{day}$, with only partial attenuation of opioid effects during maintenance on $8 \mathrm{mg} /$ day or lower. Partial attenuation of $6 \mathrm{mg}$ hydromorphone challenge was observed for up to five days after discontinuation of $8 \mathrm{mg} /$ day buprenorphine treatment. These results support clinical utility in treatment of opioid abuse and have implications for selection of effective doses and dosing frequency.

Antagonist properties in dependent subjects: Buprenorphine at doses up to $8 \mathrm{mg}$ has failed to precipitate withdrawal responses either in subjects maintained on morphine at doses up to $120 \mathrm{mg}$ /day i.m. or in subjects maintained on $30 \mathrm{mg}$ /day of oral methadone. Only in subjects maintained on 60 $\mathrm{mg}$ /day oral methadone has precipitated withdrawal been observed following acute buprenorphine administration. These findings suggest antagonist effects are limited and support the safety and acceptability of clinical transitions from street heroin or low dose methadone to buprenorphine treatment.

Physical dependence: Buprenorphine is thought to have low physical dependence potential; in animal studies it has been difficult to precipitate withdrawal responses using naloxone challenge. Only limited data are available thus far for humans, but it appears there are conditions under which both spontaneous and precipitated withdrawal can be observed. More research is needed on this topic. 
Overall, a great deal has been learned in a relatively short time about the clinical pharmacology of buprenorphine and its potential clinical utility. Like methadone, buprenorphine produces long-acting mu agonist effects when given acutely. and attenuates effects of short-acting opioids when given chronically. The dose-effect ceiling makes buprenorphine a safer medication than methadone, but purported differences in physical dependence profiles need further evaluation.

\section{AFFILIATION}

Behavioral Pharmacology Research Unit, Johns Hopkins University School of Medicine, Baltimore, Maryland

\section{BUPRENORPHINE AS A DETOXIFICATION AGENT}

\section{R. E. Johnson}

The effectiveness of buprenorphine as a detoxification agent depends on two pharmacologic characteristics: 1) the ability to suppress emergent opioid withdrawal signs and symptoms in dependent individuals, and 2) the withdrawal signs and symptoms produced by the discontinuation of buprenorphine. These two issues are intertwined when buprenorphine is tested as a medication for detoxification treatment.

Suppression Of Opiate Withdrawal Signs And Symptoms By Buprenorphine: Suppression of the opiate withdrawal syndrome by buprenorphine has not been demonstrated in a placebo-controlled study. However, studies comparing the emergence of an opioid withdrawal syndrome following the substitution of buprenorphine indicate that: 1) buprenorphine will substitute for heroin and methadone during dose induction; 2) substitution for heroin may be more easily accomplished than for methadone; 3) buprenorphine administered for a short period of time appears more effective than clonidine; and 4) buprenorphine may be effective across a wide dose range.

\section{Withdrawal Signs And Symptoms Following Dicontinuation Of Buprenorphine: In two studies where non-dependent subjects were maintained on buprenorphine and then withdrawn, autonomic signs and subjective symptoms of withdrawal have been reported as absent or minimal. In subjects dependent on heroin or methadone and treated with buprenorphine, abrupt termination of buprenorphine has been followed by the administration of placebo or an opiate antagonist. In both cases, "mild to moderate" opiate withdrawal symptoms were observed. In the one study, intensity of withdrawal symptoms appeared to be related to the dose of antagonist and of buprenorphine. Withdrawal symptoms following the cessation of buprenorphine have been judged severe enough to be treated with methadone. The dose of buprenorphine and the speed of dose reduction contribute to the magnitude of withdrawal signs and symptoms and illicit opioid use.}

In summary, buprenorphine is useful for opioid detoxification treatment because it substitutes for opioids and suppresses withdrawal symptoms in dependent individuals. Abrupt termination of buprenorphine is associated with fewer autonomic signs and symptoms of withdrawal than would be predicted with equal analgesic doses of morphine or methadone. However, chronic treatment with buprenorphine does produce a clinically significant withdrawal syndrome which 
may be related to dose. There are several critical variables that may impact on the overall clinical outcome and utility of buprenorphine in detoxification treatment, including: 1) severity of opiate dependence; 2) duration of maintenance on buprenorphine; 3) buprenorphine dose; and 4) duration of the detoxification schedule. Additional studies systematically assessing these variables are needed.

\section{AFFILIATION}

Behavioral Pharmacology Res. Unit, Johns Hopkins University, Baltimore, MD

\section{ROUTES OF PRIOR OPIATE ADMINISTRATION: EFFECTS ON O U T C O M E V A R I A B L E S I N THE NIDA/M D D \# 999 a BUPRENORPHINE MULTICENTER STUDY}

\section{L. Segal and J. L. Hill}

NIDA's Medications Development Division, in cooperation with the Los Angeles Addiction Treatment Research Center, sponsored a randomized, double blind, one year multicenter clinical trial in which 733 subjects were dosed to study the safety and efficacy of buprenorphine in the treatment of opiate dependence. This paper focuses on several outcome measures from the 16 week maintenance phase of the study according to subjects' preferred route of opiate administration at baseline.

We compared the results from intravenous injections (IV) with 216 snorters $(\mathrm{N}=204) /$ smokers $(\mathrm{N}-12)(\mathrm{S} / \mathrm{S})$. Not included in this analysis were 38 oral abusers, at least 26 of which were addicted to prescription analgesics--Percocet being the most common, and 15 subjects claiming preference for "other" routes.

Results: The most dramatic results were seen in the very sharp decrease in heroin craving on a visual analog scale near 80 (with 0 being no craving and 100 the worst craving ever experienced). After being on buprenorphine maintenance therapy for four weeks, craving dropped to about 36 in the IV group and to 26 in the $\mathrm{S} / \mathrm{S}$ group. Craving for cocaine, however, decreased only slightly. Regarding retention in treatment, $44 \%$ of the IV users completed the 16 week study while $63 \%$ of the $\mathrm{S} / \mathrm{S}$ completed. When comparing the percent of subjects with 13 consecutive opiate-free urines, $14.4 \%$ of the IV users and $18.5 \%$ of the $\mathrm{S} / \mathrm{S}$ were "clean" for that time period. At baseline, however, all subjects wee using opiates on a daily basis--one of the entrance criteria.

Based on these results, we suggest that: 1) Heroin craving can be reduced significantly within four weeks of initiating buprenorphine maintenance therapy;

2) Heroin addicts who prefer the IV route of administration are probably more heavily addicted and have a longer history of substance abuse than are S/S. The IV users in this study had a prior history of having been enrolled in about 4.6 times as many methadone maintenance programs as the $\mathrm{S} / \mathrm{S}$; 3) The ritual of injecting may have its own separate reward and is thought to be a potent reinforcer even in the tolerant addict. Therefore, we believe it is necessary to identify primary routes of administration in future treatment settings as well as research studies. 


\section{AFFILIATION}

National Institute on Drug Abuse, National Institutes of Health, Medications Development Division, Rockville, Maryland

\section{M P R O V I N G B U P R E N O R P H I NE O U T COM ES W I T H BEHAVIORAL TREATMENT}

\section{W. K. Bickel, L. Amass, and S. T. Higgins}

Two clinical trials assessed whether behavioral treatment would improve buprenorphine (BUP) detoxification outcomes. In the first trial, subjects received either combined behavioral and BUP detoxification or standard counseling and BUP. Patients (19 behavioral, 20 standard) were randomly assigned. During a 26 week detoxification, subjects received their BUP seven days a week at the clinic and provided urine samples three times per week which were analyzed for the presence of opioids. The behavioral treatment used the community reinforcement and contingency management approaches originally developed for the treatment of cocaine dependence (Higgins et al., 1993). The methadone style counseling was typical of methadone treatment counseling (Ball and Ross, 1991). Eighty-four percent of the behavioral treatment group and 80 percent of the standard treatment group remained in treatment for at least eight weeks. Fiftythree percent of the behavioral treatment group and 20 percent of the standard treatment group remained in treatment for the entire 26 weeks. Fifty-three percent of the behavioral treatment group and 30 percent of the standard treatment group provided eight weeks of continuous opioid abstinence. These results suggest that combined behavioral and BUP treatment produced outcomes superior to standard treatment and BUP.

In our second trial, we modified the medication schedule and enhanced the behavioral treatment in an effort to further improve outcomes. Our original behavioral treatment was compared to an enhanced behavioral treatment during a 16 week BUP detoxification in 21 randomly assigned patients per group. The enhanced program added procedures to address illicit opioid use and daily attendance to the original behavioral treatment. To address illicit opioid use, receipt of $1 / 2$ of the daily medication was contingent on opioid abstinence with bonus reinforcers available for providing three consecutive opioid-negative urine samples. Bonus reinforcers were a week of alternate-day dosing or items from a list of prosocial recreational activities. Sixty-seven percent of the enhanced behavioral treatment group and 43 percent of the original behavioral treatment group completed the 16-week program. Thirty-nine percent of the enhanced behavioral treatment group and 11 percent of the original behavioral treatment group provided eight weeks of continuous opioid abstinence. Thus, the enhanced behavioral program appeared to improve outcomes relative to our original behavioral treatment program.

Importantly, two-three times as many patients receiving the original behavioral treatment, and four times as many patients receiving the enhanced behavioral treatment, were abstinent on the last day of detoxification compared to patients receiving standard treatment. Thus, these two controlled trials demonstrate that behavioral treatments improve outcomes during BUP detoxification. 


\section{AFFILIATION:}

HBPL, Ira Allen School, 38 Fletcher Place, University of Vermont Burlington, Vermont

\section{J O I N T P R O B A B I L I T Y A S O U T C OME I N D E X F O R PHARMACOLOGY}

\section{W. Ling, C. J. Klett, and M. A. Compton}

Retention and drug use in pharmacotherapeutic trials most closely reflect medication treatment effects. Other commonly used outcome measures, like craving, side effects and adverse medical events, are intervening variables that are ultimately reflected in retention and/or drug use. Self report of use, number of consecutive days or weeks of abstinence and time to first use or relapse are but different dimensions of use shown by urine toxicology.

Retention is usually reported as the proportion of subjects remaining in treatment at a given point although sometimes subjects dropping out in early weeks are ignored. Drug use is usually reported as percent negative or positive for drugs and/or metabolites. Retention does not indicate whether patients are using drugs whereas percent clean urine fails to take into account that at each time point the results represent a different and decreasing number of patients. What is needed is a way to combine the effects of these measures. For individual patient performance we have advocated the use of the Treatment Effectiveness Score (TES). Its application in a cocaine pharmacology trial is presented elsewhere. For pharmacotherapeutic effects we propose the use of joint probability of retention and abstinence (JP). Both are attempts to obtain the combined effects of the two primary outcome variables but from two somewhat different perspectives. The TES focuses on the relative performance of a patient in a particular pharmacotherapeutic trial whereas the joint probability stresses the performance of a particular medication under study. For the joint probability of retention and abstinence we assign $\mathrm{P}_{1}$ as the probability of retention at a given time point (number of patients with clean urines $[\mathrm{Nr}] /$ total number enrolled $[\mathrm{Nt}]$ ) and $\mathrm{P}_{2}$ as the probability of abstinence for patients in treatment at the same time p;point (number of patients with clean urines $[\mathrm{Nc}] /$ number of patients remaining in treatment $[\mathrm{Nr}]$ ), the joint probability $\mathrm{P}_{1} \times \mathrm{P}_{2}=\mathrm{Nr} / \mathrm{Nt} \times \mathrm{Nc} / \mathrm{Nr}=\mathrm{Nc} / \mathrm{Nt}$ represents the joint probability of being in treatment and also not using.

We computed and compared the joint probability outcome for patients in four methadone/buprenorphine comparison studies - Johnson-ARC, SchottenfeldYale, Strain-Hopkins, Long-LAATRC - using retention and drug use data from each study. This report illustrates the usefulness of the composite index in comparing treatment across trials. The results suggest, with one exception, an apparent dose response effect for buprenorphine and methadone.

\section{ACKNOWLEDGEMENT}

Supported by NIDA Grant No. DA08260-01 and NIDA Interagency Agreement with West Los Angeles VAMC. Los Angeles, CA

\section{AFFILIATION}

Addiction Treatment Research Center, Los Angeles, California 


\title{
BIOLOGICAL EVALUATION OF COMPOUNDS FOR THEIR PHYSICAL DEPENDENCE POTENTIAL AND ABUSE LIABILITY. XVIII. DRUG EVALUATION COMMITTEE OF THE COLLEGE ON PROBLEMS OF DRUG DEPENDENCE, INC. (1994)
}

\author{
A. E. Jacobson
}

\section{PURPOSES OF THE DRUG EVALUATION COMMITTEE (DEC)}

The DEC was founded to ensure the continuation of the drug evaluation program, a major program of the CPDD which has been maintained as a public service from the CPDD's inception in 1928 as a committee of the National Research Council of the National Academy of Sciences. The DEC manages the evaluation carried out by a consortium of university-based groups. The researchers determine the physical dependence potential and abuse liability of drugs, and establish new, or improve older, methodology. The initial program was highly dependent on the interdisciplinary work of chemists, pharmacologists and clinicians, focusing on the synthesis and appraisal of potent analgesics which might lack the undesirable side-effects of morphine. The contemporary design of the DEC is somewhat different. The pharmacologica techniques have expanded well beyond what was originally envisioned, and the new drugs which are examined are obtained from domestic and foreign universities, pharmaceutical industry, governmental units, the World Health Organization, and other sources. Our program now also includes testing and research on stimulants and depressants.

Data obtained from the various groups associated with the DEC have provided essential information for the regulation of these drugs by governmental agencies and the World Health Organization, and are invaluable for scientists involved with the chemistry, pharmacology and clinical utility of drugs with potential abuse problems, as well as those who seek to explain the function and interaction of these drugs with CNS receptors on the molecular level. The theoretical exploration of the interaction of opioids with their receptors, as well as experimentally in binding assays, has recently become possible due to the cloning of the cDNA's of all three opioid receptors and their sequencing. ${ }^{1-8}$ The data obtained by DEC will play an important role in that research.

\section{MEMBERS OF THE DEC}

The DEC, under the chairmanship of Dr. T. Cicero (Washington University, St. Louis), has three representatives from the CPDD Board of Directors who are appointed by the President of the Board of the CPDD. These are Drs. L. Cook (DuPont Merck Pharmaceutical Co.), S. Holtzman (Emory University School of Medicine), and J. Smith (Bowman Gray School of Medicine). Each of the testing groups involved with the work of the DEC have one member on the DEC: Drs. L. Harris and G. Patrick (Medical College of Virginia), G. Winger and J. Woods (University of Michigan), and W. Woolverton (University of Mississippi Medical Center). I serve as Biological Coordinator for the DEC, continuing the work of Drs. Everette May and Nathan Eddy, the latter having initiated the coordination of the program more than 40 years ago. From its inception, this program has been coordinated in the Laboratory of Medicinal Chemistry of the National Institute of Diabetes and Digestive and Kidney Diseases (NIDDK), NIH, although there have been several organizational name changes over the decades. 


\section{STATISTICS}

Of the 42 drugs which were evaluated this year as analgesics by either or both Aceto et al., ${ }^{9}$ and Woods et al., ${ }^{10}$ three came from the U.S. and foreign pharmaceutical industry (7\%), 23 from U.S. and foreign universities (55\%), 10 from a non-profit institute (24\%), and six from NIH Intramural researchers $(14 \%)$. Comparison of the sources and numbers of compounds over a five year period is shown graphically in Figure 1. The fluctuations in the source of compounds over the past five years can be seen in that figure. The total number of compounds annually evaluated show modest changes (from 42 to 65 - a mean of $51( \pm 9)$ ). For the last several years the major source of compounds has been from universities or from a combination of universities and non-profit institutions. It is likely that this trend will continue for the next several years.

\section{FIGURE 1. DEC ANALGESIC PROGRAM. SOURCES AND NUMBER OF COMPOUNDS FROM 1990-1994}

\% From Industry - Domestic and Foreign

\% From Universities - Domestic and Foreign

\% From Other Sources - Non-profit Institutions, DEC

\% From Government - NIDA, NIH, DEA, U.S. Army

$\square$ Total Number of Compounds

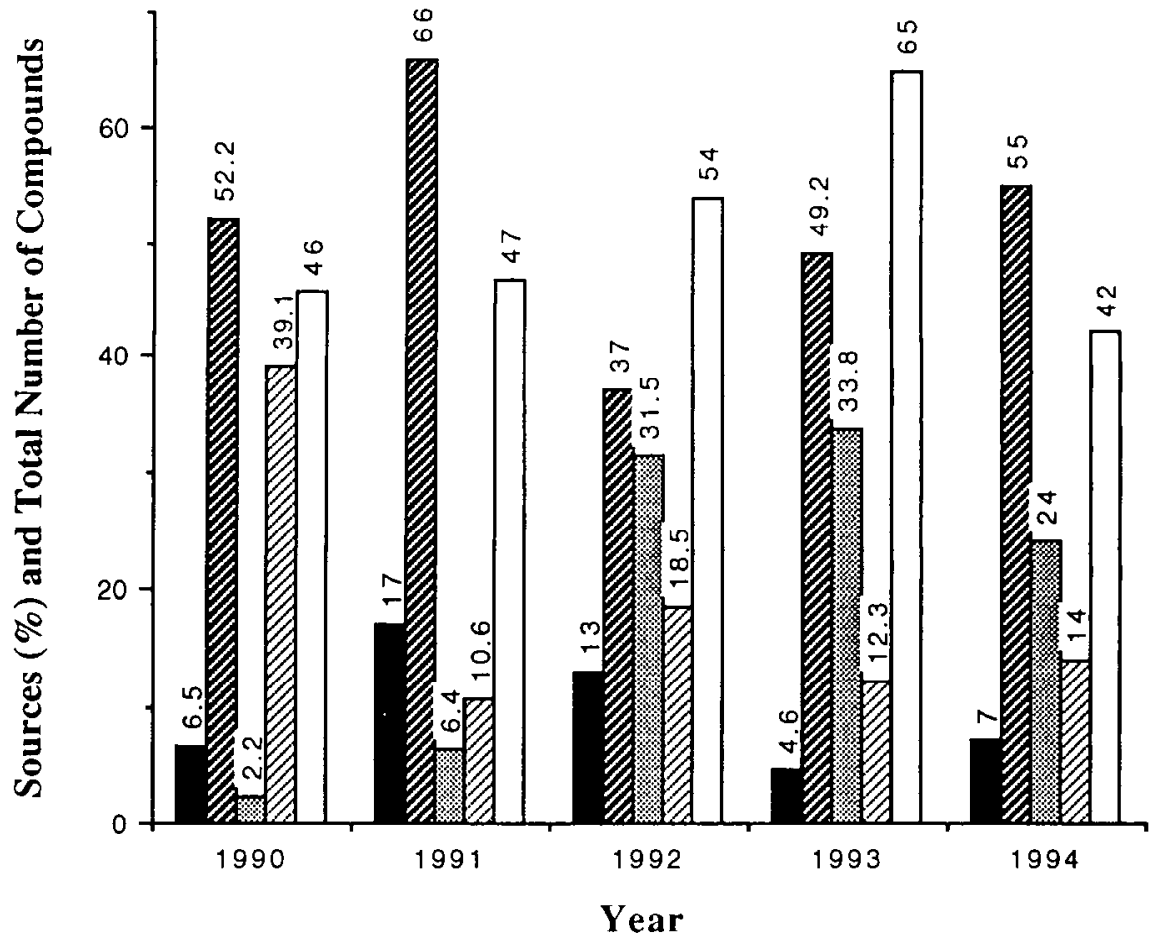

\section{PROCEDURES FOR EVALUATION OF DRUGS}

The various procedures which are used to determine the physical dependence potential and abuse liability of analgesics, depressants and stimulants have been noted heretofore, ${ }^{11}$ and are summarized in the papers by Drs. Aceto et al., ${ }^{12}$ Woods et al., ${ }^{13}$ and Patrick et al. ${ }^{14}$ 


\section{SURVEY OF EVALUATED COMPOUNDS}

\section{1) Analgesics}

The chemical names for all of the drugs which were evaluated this year can be found in table 1, and the group which evaluated the drug is also indicated therein. In order to more easily discern the biological effect of structural changes in a basic molecular structure, the examined drugs were grouped in structural classes in tables 2-12 with a summarization of the DEC-derived biological data. More comprehensive data on the individual drugs can be obtained from the reports of the members of the DEC. ${ }^{9,10}$ The tables note the biological data obtained on these drugs in previous years and indicate where those data can be found.

Seven 4,5-epoxymorphinans are shown in tables 2 and 3. The four compounds in table 2 represent two pairs of epimers with different substituents at C-6 (NIH 10683 and 10684, and 10701 and 10702). The biological properties of the latter two are similar, but 10683 and 10684 are distinctly different. Depending on the assay, NIH 10683 might be considered a $\mu$ - or $\boldsymbol{\kappa}$-agonist. It does not show antagonist properties in antinociceptive studies or in the monkey analgesia study. $\mathrm{NIH} 10683$ does not substitute for morphine in the SDS assay; it does not act as a $\mathrm{u}$-agonist, but rather acts as a non-selective antagonist in the vas deferens preparation. In drug discrimination it acts as a $\kappa$-agonist and not as a $\mu$-agonist or antagonist, and in self administration NIH 10683 substitutes for alfentanil, a $\mu$ agonist. It suppresses respiration in the respiratory function assay in monkeys, like other $\mu$-agonists. Its $\mathrm{C}-6 \boldsymbol{\alpha}-\mathrm{OH}$ epimer, $\mathrm{NIH} 10684$, appears to have $\mu$ antagonist or perhaps $\mu$-agonist properties, depending on the assay. It has agonist activity in the PPQ assay but not in other assays in mice or monkey. It is seen as a $\mu$-selective antagonist in the vas deferens, precipitates withdrawal with a slow onset and long duration of action in SDS in monkeys, and shows antagonist properties in drug discrimination. NIH 10684 maintains self-injection in the monkey, but it was less efficacious than alfentanil, a $\mu$-agonist. All four of the compounds have good affinity for the opioid receptors. The bulky iodine atom at C-6- $\beta$ and $-\alpha$ for 10701 and 10702, respectively, appears to have little effect on its binding to $\mu, \kappa$ and $\delta$ receptors.

NIH 10773 (table 3) bears a sulfate ester at C-6 and is morphine-like in antinociceptive potency in the mouse. However, it does not substitute for morphine in SDS, appears as a weak agonist in the vas deferens, and does not bind well to opioid receptors. The compound exists as a zwitterion, possessing structural characteristics reminiscent of an amino acid, yet appears to easily pass through the blood-brain barrier in vivo.

The substituents at C-1 and C-2 in NIH 10787 and 10814 (table 3) might have been expected (from the work of others) to interfere with biological activity. However, NIH 10787 was found to be a very potent antagonist and 10814, which had less affinity for the opioid receptors than 10787, was noted to have insurmountable antagonist actions at the $\mathrm{\kappa}$ receptor.

Table 4 lists the actions of six endoetheno- and endoethanooripavines. NIH 10805 has a chlorine atom at C-1, like NIH 10787 (table 3) and acts as a potent antagonist. NIH 10812, however, a 2-nitro-substituted buprenorphine, does not have opioid actions in vivo. NIH 10813 appears to be very similar to 10814 (table 3) in binding and in the vas deferens preparation. The in vivo work on most of the compounds in table 4 is scheduled for 1994.

Four rather inactive (+)-morphinans are listed in table 5, as is a (-)-morphinan (NIH 10802). Structurally similar molecules have affinity for sigma $(\sigma)$ receptors 
or PCP binding sites. SDS data on the phenylmorphan in table 5, NIH 10779, completes the data gathered previously on that essentially morphine-like compound.

Table 6 displays the actions of four (+)- and (-)-6,7-benzomorphans. These, and other $\mathrm{N}-n$-alky-substituted 6,7-benzomorphans are among the compounds which were the subject of a paper which was recently submitted to J. Med. Chem. ${ }^{15}$ Several of the authors arc DEC members. Some of the compounds in the series were found to be among the most potent knowno receptor ligands. It is perhaps somewhat surprising to note that NIH 10675 and 10697, although they are in the (+)-benzomorphan series, have good (NIH 10675) or reasonable (NIH 10697) antinociceptive activity.

Three arylpiperidines which seem to exert antagonist activity in the vas deferens preparation are shown in table 7, and 10 compounds related to fentanyl are listed in tables 8 and 9. Most of the fentanyl-like compounds had activity in the SDS assay ranging between morphine-like to that of NIH 10792 (table 9) or 10741 (table 8) which were estimated to be 30,000 or $20,000-50,000$ times more potent than morphine, respectively. These compounds arc among the most potent known narcotic agonists.

The remaining tables 10 and 11 consider the miscellaneous compounds. NIH 10700 (table 11) is structurally similar to PCP, but has antinociceptive activity and some affinity for the opioid receptors. NIH 10815 (table 11) was seen to be relatively selective, and potent, for $\delta$-receptors in the vas deferens preparation, but did not have much affinity for opioid receptors. Other workers have indicated that it is a potent and selective $\delta$-ligand in vitro as well as in the vas deferens and guinea pig ileum preparations.

\section{2) Stimulants and Depressants}

Two compounds (stimulants) were released for publication this year (CPDD 0038 from pharmaceutical industry, and 0041, Etryptamine acetate, from NIDA). ${ }^{16}$ The molecular structures of these compounds and the summarization of the data collected on them by the Stimulant/Depressant testing groups of DEC are shown in table 12. Our data on Etryptamine and several other drugs will be used by the World Health Organization for scheduling purposes. Two or three compounds are being processed for testing in 1994-1995.

\section{ABBREVIATIONS USED IN TABLES 2 - 13}

Rounded numbers are used in the tables; $M=$ morphine. Precise values and details of the procedures are given in the $\mathrm{MCV}^{9}$ and $\mathrm{UM}^{10}$ reports; $\mathrm{NT}=$ not tested.

For "E" notation: $1 \mathrm{E}-3=1 \times 10^{-3}$ or $0.001 \mathrm{M}(1 \mathrm{mM}), 1 \mathrm{E}-6=1 \mathrm{mM}, 1 \mathrm{E}-9=1$ $\mathrm{nM}, 1 \mathrm{E}-12=1 \mathrm{pM}$ (picomole), and 1E-15 = $1 \mathrm{fM}$ (femtomole).

1) MOUSE ED50/AD50: Antinociceptive Assays (sc injection) ${ }^{9}$

Confidence limits are listed in the MCV report. ${ }^{9}$

$\mathbf{H P}=$ hot plate (morphine $\left.\mathrm{ED}_{50}=0.8(0.3-1.8)\right)$

$\mathbf{P P Q}=$ phenylquinone (morphine $\mathrm{ED}_{50}=0.23(0.20-0.25)$ )

$\mathbf{T F}=$ tail-flick (morphine $\left.\mathrm{ED}_{50}=5.8(5.7-5.9)\right)$

TFA = tail-flick antagonism vs. morphine (naltrexone $\mathrm{AD}_{50}=0.007(0.002-$ $0.02)$; naloxone $\mathrm{AD}_{50}=0.035$ (0.01-0.093)). 

$\mathrm{I}=$ inactive, without a reasonable dose-response relationship, or insufficiently active for statistical analysis.

2) IN VITRO (Data from UM) ${ }^{10}$

RBH = binding affinity in rat cerebrum membranes (displacement of $0.5 \mathrm{nM}$

$\left[{ }^{3} \mathrm{H}\right]$ etorphine) in the presence of $150 \mathrm{mM} \mathrm{NaCl}$ (morphine $\mathrm{EC}_{50}=23.6$ ).

$\mathrm{NE}=$ no effect.

NOTE: Contemporary $\mathrm{EC}_{50}$ data cannot be directly compared with those from reports before $1985^{17}$ which were obtained under "-NaCl" (without $\mathrm{NaCl}$ ) conditions.

VD = electrically stimulated mouse vas deferens $\mathrm{EC}_{50}$ values, rounded to one significant figure. Partial agonist indicated by $\%$ inhibition of twitch in parenthesis; $[\mathrm{A}]=$ antagonism by naltrexone.

$\mathrm{SE}=$ slight effect on twitch.

$\mathrm{NE}=$ No significant agonist or antagonist effect.

ANT $=$ Antagonist activity. Selective antagonist activity at $\mu$, $\delta$, and/or $\mathrm{x}$ receptors is noted in parentheses. The antagonist effect may or may not be competitive.

Compounds which suppress the twitch and are not antagonized by naltrexone or other narcotic antagonists are said to be non-opioid agonists (e.g., clonidine, a non-opioid agonist, can suppress the twitch but is not antagonized by naltrexone). Compounds which bind with reasonable affinity in the RBH assay and do not suppress the twitch in the VD may have narcotic antagonist properties. The opioid receptor at which the drug exerts its antagonist effect is determined by testing various concentrations of the drug to induce a blockade (antagonism) of the suppression of the twitch in the VD preparation caused by sufentanil $(\mu)$, DSLET $(\delta)$ or U50,488 $(\kappa){ }^{10}$

3) IN VIVO: in the rhesus monkey (from $\mathrm{MCV}^{9}$; from MCV or UM prior to 1988).

$$
\begin{aligned}
\text { SDS }= & \text { single-dose-suppression } \\
\text { NS }= & \text { no suppression } \\
\mathrm{CS}= & \text { complete suppression } \\
\mathrm{PS}= & \text { partial suppression } \\
& \quad \text { (Parenthesized numbers }=\text { dose range studied, in } \mathrm{mg} / \mathrm{kg} \text { ) }
\end{aligned}
$$

Other Studies (noted in the footnotes to the tables)

A) In Rat:

$\mathbf{R I}=$ rat continuous infusion (data from $\mathrm{MCV})^{9}$

1) $\mathbf{S M}=$ substitution for morphine

$\mathrm{NS}=$ no substitution for morphine

$\mathrm{CS}=$ complete substitution

PS $=$ partial substitution

2) $\mathbf{P P D}=$ primary physical dependence

B) In Rhesus Monkey:

1) $\mathbf{P P t}-\mathbf{W}=$ studies in non-withdrawn monkeys (data from MCV) ${ }^{9}$

$\mathrm{PW}=$ precipitated-withdrawal at dose levels, in $\mathrm{mg} / \mathrm{kg}$, indicated in parentheses \&/or comparison with naloxone $[\mathrm{N}]$.

$\mathrm{SP}=$ slight precipitation

$\mathrm{NP}=$ no precipitation

2) $\mathbf{N D}=$ studies using non-dependent monkeys (data from $\mathrm{MCV})^{9}$ 
M-like $=$ morphine-like effect.

3) $\mathbf{P P D}=$ primary physical dependence $(\text { data from } \mathrm{MCV})^{9}$

4) $\mathbf{S A}$ or SI $=$ self-administration or self-injection $(\text { data from } \mathrm{UM})^{10}$

$\mathrm{NE}=$ no effect

High $=$ codeine-like

$\mathrm{IN}=$ intermediate between saline and codeine

$\mathrm{SE}=$ slight effect

5) $\mathbf{D D}=$ drug discrimination $\left(\right.$ data from UM) ${ }^{10}$

$\mathrm{NE}=$ no effect

$\mathrm{CS}=$ complete substitution

6) $\mathbf{M A}=$ monkey analgesia (data from $\mathrm{UM})^{10}$

7) $\mathbf{R F}=$ respiratory function (data from UM) ${ }^{10}$

C) In Vitro (data from UM) ${ }^{10}$

BIND - binding affinity using monkey brain cortex membranes (selectivity for $\mu, \kappa$, and $\delta$ opioid receptors determined with $\left[{ }^{3} \mathrm{H}\right]$ sufentanil, $\left[{ }^{3} \mathrm{H}\right]$-DPDPE and $\left[{ }^{3} \mathrm{H}\right]-\mathrm{U} 69,593$, respectively).

\section{Previous Reports}

Previous work on a compound is noted using the year listed in the monograph title (e.g., work cited as "1992" indicates that the work was included in "Problems of Drug Dependence 1992", which was published in 1993). Note that the monograph's publication date may be one year after the titled year of the monograph. Complete details of the original work on a compound can be found in the Annual Report from either UM or MCV. 
TABLE 1. NIH NUMBERS, CHEMICAL NAMES, TABLE NUMBER, AND EVALUATING GROUP

\begin{tabular}{|c|c|c|}
\hline NIH\# & NAME & $\begin{array}{l}\text { TABLE\#- } \\
\text { Evaluator }^{\text {a }}\end{array}$ \\
\hline 04591 & $\begin{array}{l}\text { (+)-3-Hydroxy-N-Methylmorphinan.HBr } \\
\text { (Dextrophan, Dromoran) }\end{array}$ & $5-\mathrm{MCV}^{\mathrm{a}}$ \\
\hline 10560 & $\begin{array}{l}(-)-5,9 \alpha \text {-Dimethyl-2-ethyl-2'-hydroxy-6,7- } \\
\text { benzomorphan.HCl }\end{array}$ & 6-MCV \\
\hline 10675 & $\begin{array}{l}(-)-5,9 \alpha \text {-Dimethyl-2- } n \text {-heptyl-2'-hydroxy-6,7- } \\
\text { benzomorphan.HCl }\end{array}$ & 6-MCV \\
\hline 10683 & $\begin{array}{l}\text { 14-Hydroxy-N-(2-methoxyethyl)-7,8- } \\
\text { dihydronomorphine }\end{array}$ & $2-\mathrm{MCV} / \mathrm{UM}^{\mathrm{b}}$ \\
\hline 10684 & $\begin{array}{l}\text { 14-Hydroxy-N-(2-methoxyethyl)-7,8- } \\
\text { dihydronorisomorphine }\end{array}$ & 2-MCV/UM \\
\hline 10697 & $\begin{array}{l}(-) 5,9 \alpha \text {-Dimethyl-2'-hydroxy-2- } n \text {-octyl-6,7- } \\
\text { benzomorphan. } \mathrm{HCl}\end{array}$ & 6-UM \\
\hline 10698 & $\begin{array}{l}(-) 5,9 \alpha \text {-Dimethyl-2'-hydroxy-2- } n \text {-octyl-6,7- } \\
\text { benzomorphan. } \mathrm{HCl}\end{array}$ & 6-UM \\
\hline 10700 & $\begin{array}{l}\text { 1-[-(2-Hydroxyphenyl)cyclohexyl]-3,4- } \\
\text { dehydroperidine.HCl }\end{array}$ & $10-\mathrm{UM}$ \\
\hline 10701 & 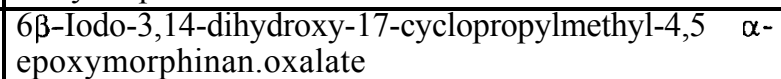 & $2-\mathrm{UM}$ \\
\hline 10702 & 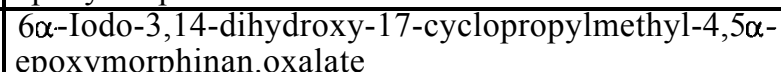 & $2-U M$ \\
\hline 10703 & $\begin{array}{l}\mathrm{N}-[(3,4-\text { Diclorophenyl)acetyl]-N,2-dimethyl-2- } \\
\text { (N',N'-dimethylamino)ethylamine.oxalate }\end{array}$ & $10-\mathrm{UM}$ \\
\hline 10705 & $\begin{array}{l}\mathrm{N} \text {-(n-Propyl)-N'-3,4-dichlorophenylethyl) } \\
\text { piperazine. } 2 \mathrm{HBr}\end{array}$ & 10 -UM \\
\hline 10735 & (+)N-Benzyl-3-hydroxymorphinan.HCl & $5-\mathrm{MCV}$ \\
\hline 10738 & $\begin{array}{l}\text { 4-(3-Hydroxyphenyl)-1-(4-nitrobenzyl)-4-(1- } \\
\text { oxopropyl)piperidine.HCl }\end{array}$ & 7-MCV \\
\hline 10739 & $\begin{array}{l}\text { 1-(4-Fluorobenzyl)-4-(3-hydroxybenzyl)-4-(1- } \\
\text { oxopropyl)piperidine.HCl }\end{array}$ & 7-MCV \\
\hline 10741 & 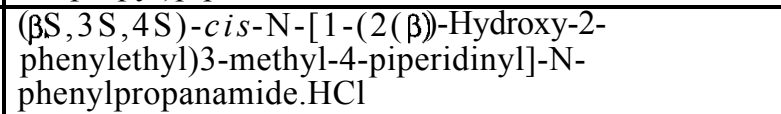 & $8-\mathrm{MCV}$ \\
\hline 10749 & (+)-3-Hydroxy-N-(4-nitrobenzyl)morphinan & 5-MCV \\
\hline 10762 & $\begin{array}{l}\text { ( } \pm \text { )-c i s-N-[3-Methyl[2-oxo-2-(2-thienyl)ethyl]-4- } \\
\text { piperidinyl]-N-phenylpropanamide.HCl }\end{array}$ & 8-UM \\
\hline 10765 & $\begin{array}{l}( \pm)-c i s-\mathrm{N}-[1-(2-\mathrm{Hydroxy}-1 \text {-phenylethyl)-3-methyl-4- } \\
\text { piperidinyl]-N-phenylpropanamide.HCl }\end{array}$ & 8-UM \\
\hline 10773 & Morphine-3-acetate-6-sulfate (zwitterion) & 3-MCV/UM \\
\hline 10779 & 2,3-Dimethyl-5-(3-hydroxyphenyl)morphan. $\mathrm{HBr}$ & 5-MCVIUM \\
\hline 10782 & Rifampin (Rifampicin) & 10-MCV/UM \\
\hline 10784 & $\begin{array}{l}\text { ( } \pm \text { )-cis-N-[1-(2-Hydroxy-2-phenylethyl)-3-methyl-4- } \\
\text { piperidinyl]-N-(3-fluorophenyl)propanamide.HCl }\end{array}$ & $8-\mathrm{MCV}$ \\
\hline 10785 & $\begin{array}{l}\text { ( } \pm \text { )-cis-N-[1-(2-Hydroxy-2-phenylethyl)-3-methyl-4- } \\
\text { piperidinyl]-N-(4-fluorophenyl)propanamide.HCl }\end{array}$ & 8-MCV/UM \\
\hline 10786 & $\begin{array}{l}\text { ( } \pm \text {-cis-N-[1-[2-(4-Bromophenyl)(ethyl-2-hydroxy]-3- } \\
\text { methyl-4-piperidinyl]-N-phenylpropanamide. } \mathrm{HCl}\end{array}$ & 9-MCV/UM \\
\hline 10787 & 1-Chloronaltrexone. $\mathrm{HCl}$ & 3-MCV/UM \\
\hline
\end{tabular}


TABLE 1 (CONTINUED). NIH NUMBERS, CHEMICAL NAMES, TABLE NUMBER, AND EVALUATING GROUP

\begin{tabular}{|c|c|c|}
\hline 10790 & $\begin{array}{l}\text { ( } \pm \text {-1-(2-Hydroxy-2-phenylethyl)-t-3-methyl-4-[(1- } \\
\text { oxopropyl)phenylamino]-r-4-piperidinecarboxamide } \\
\text { Methyl Ester.HCl }\end{array}$ & 9-MCV/UM \\
\hline 10791 & $\begin{array}{l}\text { ( } \pm \text {-1-(2-Hydroxy-2-phenylethyl)-c-3-methyl-4-[(1- } \\
\text { oxopropyl)phenylamino]-r-4-piperidinecarboxamide } \\
\text { Methyl Ester.HCl }\end{array}$ & 9-MCV/UM \\
\hline 10792 & $\begin{array}{l}\text { ( } \pm \text {-1-(2-Hydroxy-2-phenylethyl)-t-3-methyl-4-[(1- } \\
\text { oxopropyl)phenylamino]-r-4-piperidinecarboxamide } \\
\text { Ethyl Ester.HCl }\end{array}$ & 9-MCV/UM \\
\hline 10793 & $\begin{array}{l}( \pm)-\mathrm{N}-[1-(2-H y d r o x y-2-\text { phenylethyl })-4- \\
\text { methoxymethyl-c-3-methyl-r-4-piperidinyl]-N- } \\
\text { phenylpropanamide oxalate }\end{array}$ & 9-MCV/UM \\
\hline 10794 & Amitriptylene. $\mathrm{HCl}$ & 11-MCV/UM \\
\hline 10795 & $\begin{array}{l}\text { 4-(3-Hydroxyphenyl-4-(1-oxopropyl)-1-(2-methyl-2- } \\
\text { butenyl) piperidine. } \mathrm{HCl}\end{array}$ & 7-MCV \\
\hline 10796 & $(+)-\mathrm{N}-(2-$-Methylpropenyl)-3-hydroxymorphinan. $\mathrm{HBr}$ & 5-MCV \\
\hline 10802 & (-)-3-Hydroxy-N-(4-hydroxybenzyl)morphinan. $\mathrm{HBr}$ & 5-MCV/UM \\
\hline 10803 & $( \pm)$-N-Allylmecamylamine. $\mathrm{HCl}$ & $11-\mathrm{MCV} / \mathrm{UM}$ \\
\hline 10805 & 1-Chlorodiprenorphine oxalate & 4-MCV/UM \\
\hline 10809 & $\begin{array}{l}\text { N-Cyclopropylmethyl }\left[7 \alpha_{n} 8 \alpha, 2^{\prime}, 3^{\prime}\right] \text { cyclopentano-I'- } \\
{[R] \text { hydroxy-6,14-endoethenotetrahydronororipavine }} \\
\text {.HCl }\end{array}$ & 4-MCV/UM \\
\hline 10810 & $\begin{array}{l}\left.\text { N-Cyclopropylmethyl[7 } \alpha, i^{\prime} 8 \alpha, 2^{\prime}, 3^{\prime}\right] \text { cyclopentano-1'- } \\
{[S] \text { hydroxy-6,14-endoethenotetrahydronororipavine }} \\
. \mathrm{HCl}\end{array}$ & 4-UM \\
\hline 10811 & $\begin{array}{l}\text { N-Cyclopropylmethyl }\left[7 \alpha, 8 \alpha, 2^{\prime}, 3^{\prime}\right] \text { cyclohexano-1'- } \\
{[S] \text { hydroxy-6,14-endoethenotetrahydronororipavine }} \\
\text {.HCl }\end{array}$ & 4-UM \\
\hline 10812 & 2-Nitrobuprenorphine.HCI & 4-UM \\
\hline 10813 & 2-Nitrodiprenorphine.HCI & 4-UM \\
\hline 10814 & 2-Nitronaltrexone.HCl & 3-UM \\
\hline 10815 & $\begin{array}{l}(+)-4-[(\alpha R) \alpha-(12 S, 5 R)-4-A l l y l-2,5-\text { dimethyl-1- } \\
\text { piperazinyl)-3-methoxybenzyl]-N,N- } \\
\text { diethylbenzamide }\end{array}$ & 11-UM \\
\hline $\begin{array}{l}\text { CPDD } \\
0038\end{array}$ & $\begin{array}{l}\text { 2-(7-Chloro-1,8-naphthyridin-2-yl)-3-[(1,4-dioxa-S- } \\
\text { azaspiro[4.5]dec-8-yl)carbonylmethyl]isoindolin-1- } \\
\text { one }\end{array}$ & $\begin{array}{l}\text { 12-MCV/UM } \\
/ \mathrm{UMSb}\end{array}$ \\
\hline $\begin{array}{l}\text { CPDD } \\
0041\end{array}$ & Etryptamine acetate & $\begin{array}{l}\text { 12-MCV/ } \\
\mathrm{UM} / \mathrm{UMSb}\end{array}$ \\
\hline
\end{tabular}

a) For complete data from MCV, see reference 13, and see reference 14 for complete data from UM.

b) See reference 16 for complete data from MCV, UM, and UMS. 


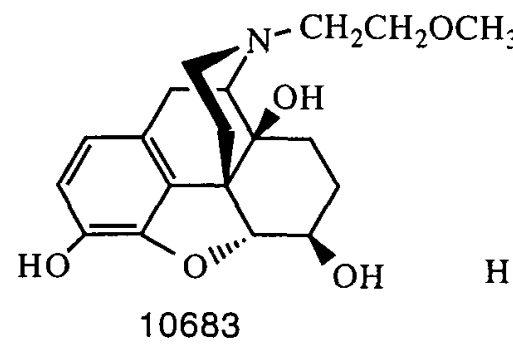

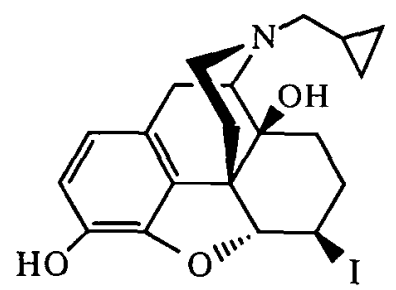

10701
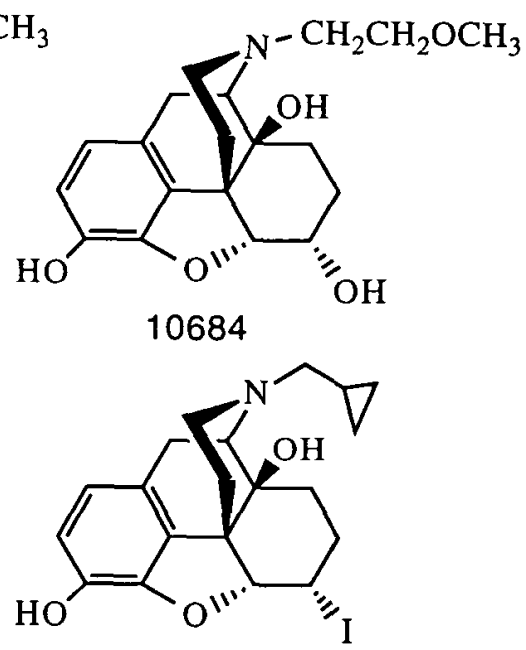

10702

MOUSE ED50/AD50

IN VITRO

MONKEY

\begin{tabular}{|l|l|l|l|l|l|l|l|}
\hline NIH \# & $\underline{\text { HP }}$ & PPQ & T F & $\underline{\text { TFA }}$ & $\underline{\text { RBH }}$ & $\underline{\text { VD }}$ & $\underline{\text { SDS }}$ \\
\hline & & & & & & & \\
\hline 10683 & 0.3 & $0.03^{\mathrm{b}}$ & $0.2^{\mathrm{b}}$ & $\mathrm{I}$ & $8.2 \mathrm{nM}$ & $7 \mathrm{E}-6[\mathrm{NA}]^{\mathrm{c}}$ & $\mathrm{NS}(0.5-6)^{\mathrm{d}}$ \\
\hline 10684 & $\mathrm{I}$ & 2.6 & $\mathrm{I}$ & $\mathrm{I}$ & $5.4 \mathrm{nM}$ & $4.9 \mathrm{E}-5[\mathrm{NA}]^{\mathrm{e}}$ & $\mathrm{NS}(1,4)^{\mathrm{t}}$ \\
\hline 10701 & $\mathrm{I}^{\mathrm{g}}$ & $\mathrm{I}^{\mathrm{g}}$ & $\mathrm{I}^{\mathrm{g}}$ & $0.08^{\mathrm{g}}$ & $5.2 \mathrm{nM}^{\mathrm{g}, \mathrm{h}}$ & $\mathrm{ANT}^{\mathrm{g}, \mathrm{i}}$ & $\mathrm{NS}^{\mathrm{g}, \mathrm{j}}$ \\
\hline 10702 & $\mathrm{I}^{\mathrm{g}}$ & $\mathrm{I}^{\mathrm{g}}$ & $\mathrm{I}^{\mathrm{g}}$ & $0.04^{\mathrm{g}}$ & $2.1 \mathrm{nM}^{\mathrm{g}, \mathrm{k}}$ & $\mathrm{ANT}^{\mathrm{g}, 1}$ & $\mathrm{~N} \mathrm{~S}^{\mathrm{g}, \mathrm{m}}$ \\
\hline
\end{tabular}

a) See text for explanation of column headings and abbreviations.

b) AD50 (naloxone vs ED80) - PPQ: 0.6; - TF: 0.03 .

c) Not $\mu$-like agonist; $\mu, \delta, \kappa$-antagonist.

d) Not $\mu$-like in monkey; MA: analgesic[A]; SA: substitutes for alfentanil (0.03 $\mathrm{mg} / \mathrm{kg} / \mathrm{inj}$ ); DD: $\kappa$--agonist, not $\mu$-agonist or antagonist; RF: suppression[A].

e) $\mu$-Selective antagonist.

f) PPt-W: agonist-antagonist with slow onset and long duration; DD: no agonist effects, substituted for naltrexone; MA: NE (antagonist); RF: NE;

SA: maintained self-injection in $2 / 3$ monkeys, less efficacious than alfentanil.

g) Previously reported -1992.

h) BIND : $\mu=1.06 \mathrm{nM}, \delta=26 \mathrm{nM}, \kappa:=0.34 \mathrm{nM}$

i) Non-selective.

j) PPt-W - PW.

k) BIND: $\mu=0.42 \mathrm{nM}, 6=9.6 \mathrm{nM}, \kappa=0.3 \mathrm{nM}$

1) $\mu$-Selective.

m) Exacerbates withdrawal. 

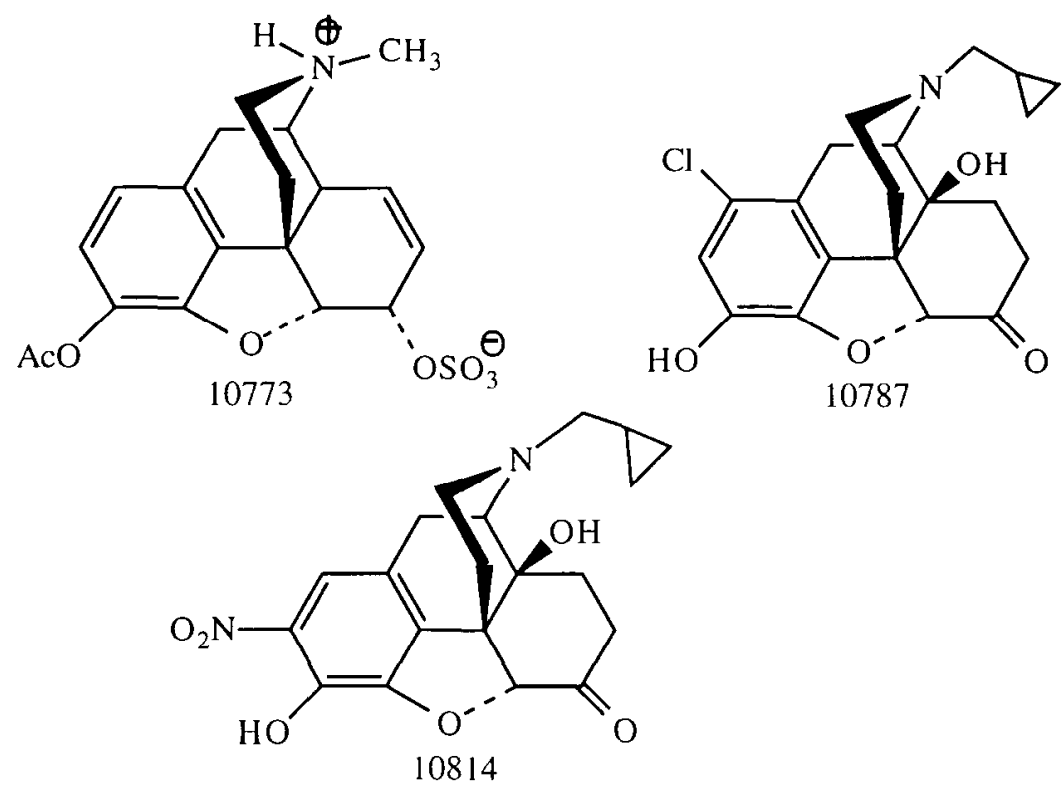

10787

MOUSE ED50/AD50

IN VITRO

MONKEY

\begin{tabular}{|l|l|l|l|l|l|l|l|}
\hline$\underline{\mathrm{NIH}} \#$ & $\underline{\mathrm{HP}}$ & $\underline{\mathrm{PPQ}}$ & $\underline{\mathrm{TF}}$ & $\underline{\mathrm{TFA}}$ & $\underline{\mathrm{RBH}}$ & $\underline{\mathrm{VD}}$ & $\underline{\underline{\text { SDS }}}$ \\
\hline 10773 & 1.0 & 0.5 & 2.5 & $\mathrm{I}$ & $517 \mathrm{nM}$ & $\begin{array}{l}139 \\
\mathrm{nM}(81)[\mathrm{A}]^{\mathrm{b}}\end{array}$ & $\begin{array}{l}\mathrm{NS} \\
(2.5,10)\end{array}$ \\
\hline 10787 & $\mathrm{I}$ & $\mathrm{I}^{\mathrm{I}}$ & $\mathrm{I}^{\mathrm{c}}$ & $4.8 \mathrm{E}-4$ & $0.38 \mathrm{nM}$ & $\mathrm{ANT}^{\mathrm{d}}$ & $\begin{array}{l}\mathrm{NS} \\
(0.0016)^{\mathrm{e}}\end{array}$ \\
\hline 10814 & - & - & - & - & $196 \mathrm{nM}$ & $3.5 \mathrm{E}-9(23)[\mathrm{A}]^{\mathrm{f}}$ & - \\
\hline
\end{tabular}

a) See text for explanation of column headings and abbreviations.

b) Weak partial agonist.

c) $\mathrm{pA}_{2}$ vs $\mathrm{M}=8.7, \mathrm{pA}_{2}$ vs $10672(\kappa)=$ complete antagonism.

d) Potent antagonist, slightly selective for $\mu$-receptors.

e) PPt-W: PW [5 x naloxone].

f) Competitive antagonist at $\mu$ and $\delta$, insurmountable antagonist at $\kappa$. 


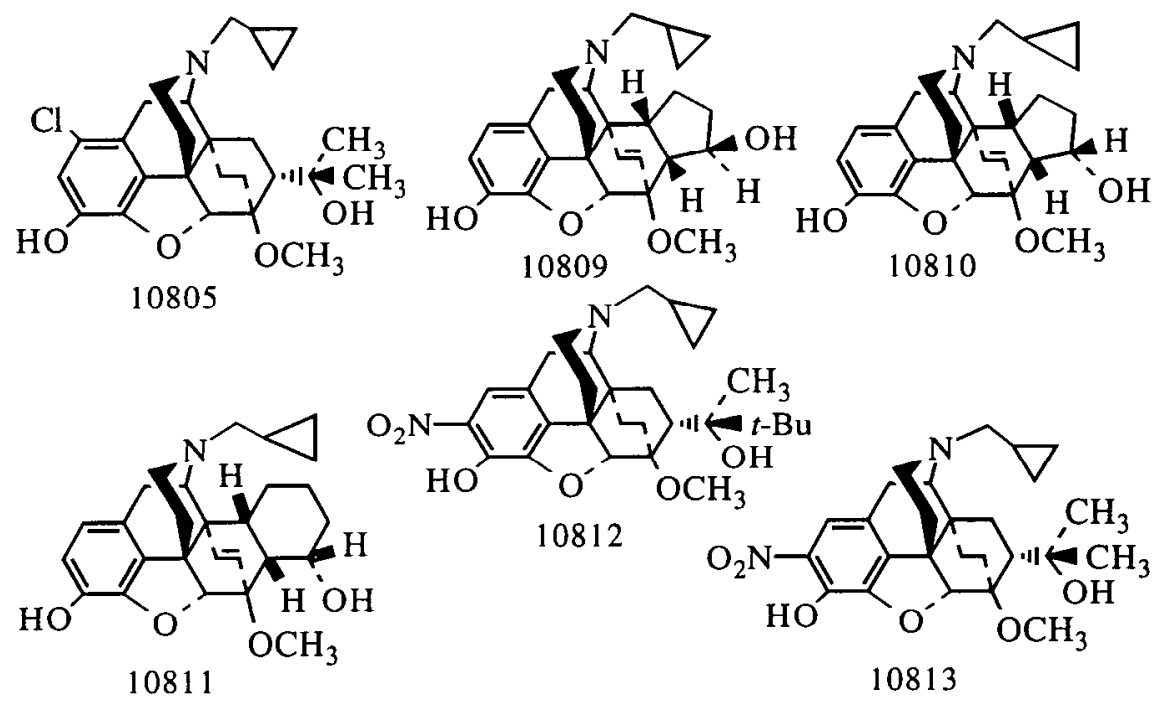

MOUSE ED50/AD50

IN VITRO

MONKEY

\begin{tabular}{|l|l|l|l|l|l|l|c|}
\hline$\underline{\text { NIH \# }}$ & $\underline{\text { HP }}$ & $\underline{\text { PPQ }}$ & $\underline{\text { T F }}$ & $\underline{\text { TFA }}$ & $\underline{\text { RBH }}$ & $\underline{\text { VD }}$ & $\underline{\text { SDS }}$ \\
\hline & & & & & & & \\
\hline 10805 & I & I & I & $0.09^{\mathrm{b}}$ & $0.61 \mathrm{nM}$ & ANT $^{\mathrm{c}}$ & $\begin{array}{l}\text { NS } \\
(0.01-0.02)^{\mathrm{d}}\end{array}$ \\
\hline 10809 & I & 0.01 & 0.1 & 9.3 & $0.49 \mathrm{nM}$ & $2.2 \mathrm{E}-9(74)[\mathrm{A}]^{\mathrm{e}}$ & $\begin{array}{l}\mathrm{N} \mathrm{S} \\
(0.05-0.025)^{\mathrm{d}, \mathrm{f}}\end{array}$ \\
\hline 10810 & - & - & - & - & $0.91 \mathrm{nM}$ & $8.8 \mathrm{E}-9(78)[\mathrm{A}]^{\mathrm{g}}$ & - \\
\hline 10811 & - & - & - & - & $0.59 \mathrm{nM}$ & $0.86 \mathrm{E}-9(99)[\mathrm{A}]^{\mathrm{g}}$ & - \\
\hline 10812 & - & - & - & - & $3.81 \mu \mathrm{M}$ & $\begin{array}{l}6.7 \mathrm{E}- \\
9(56)[\mathrm{NA}]^{\mathrm{h}}\end{array}$ & - \\
\hline 10813 & - & - & - & - & $140 \mathrm{nM}$ & $4 \mathrm{E}-7(44)[\mathrm{A}]^{\mathrm{i}}$ & - \\
\hline
\end{tabular}

a) See text for explanation of column headings and abbreviations.

b) $\mathrm{pA}_{2}$ vs $\mathrm{M}=$ non-competitive antagonist; $\mathrm{pA}_{2}$ vs $10672(\kappa)=8.0$ (high affinity for $\boldsymbol{\kappa}$ ).

c) Non-selective; insurmountable at $\boldsymbol{\kappa}$.

d) Exacerbates withdrawal; PPt-W: PW (like naloxone).

e) Relatively selective K-agonist.

f) Longer-acting than naloxone. Partial $\mu$-agonist; perhaps $\kappa$ and $\sigma$-actions.

g) Relatively selective $\mu$-agonist.

h) Very weak mixed antagonist, slightly more potent at $\kappa$ and $\delta$.

i) Competitive antagonist at $\mu$ and $\delta$, insurmountable antagonist at $\kappa$. 


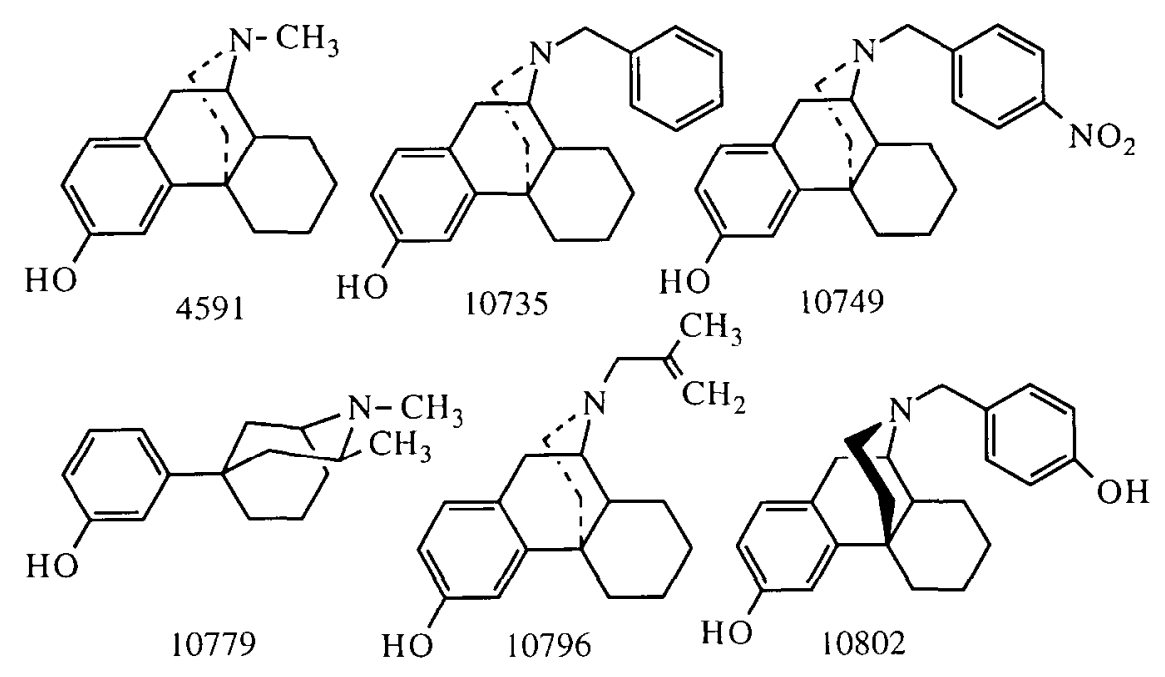

MOUSE ED50/AD50

IN VITRO MONKEY

\begin{tabular}{|c|c|c|c|c|c|c|c|}
\hline NIH \# & HP & PPQ & TF & TFA & RBH & $\mathrm{VD}$ & SDS \\
\hline 04591 & $58^{b}$ & 24 & I & I & $1.4 \mu \mathrm{M}$ & - & $\overline{P S}(4)^{c}$ \\
\hline 10735 & I & 6.1 & I & I & $>6 \mu \mathrm{M}^{\mathrm{d}}$ & $\mathrm{ANT}^{\mathrm{d}, \mathrm{e}}$ & $\operatorname{NS}(1.8,7)^{\mathrm{f}}$ \\
\hline 10749 & I & I & I & I & $>6 \mu \mathrm{M}^{\mathrm{d}}$ & Insoluble $^{\mathrm{d}}$ & NS (4.16) \\
\hline 10779 & $4.8^{\mathrm{d}}$ & $0.3^{\mathrm{d}}$ & $1.3^{\mathrm{d}}$ & $\mathrm{I}^{\mathrm{d}}$ & $592 \mathrm{nM}^{\mathrm{d}}$ & $15.8 \mu \mathrm{M}^{\mathrm{d}, \mathrm{g}}$ & $\mathrm{CS}(0.75,3)$ \\
\hline 10796 & I & 11.4 & I & I & $>6 \mu \mathrm{M}^{\mathrm{d}}$ & $\mathrm{ANT}^{\mathrm{d}, \mathrm{h}}$ & PS $(15)^{i}$ \\
\hline 10802 & I & $\mathrm{I}$ & I & $\bar{I}$ & $2.1 \mu \mathrm{M}$ & $\begin{array}{l}108 \mathrm{nM}(23) \\
{[\mathrm{NA}]^{\mathrm{j}}}\end{array}$ & $\mathrm{NT}$ \\
\hline
\end{tabular}

a) See text for explanation of column headings and abbreviations.

b) Previously reported - 1951 .

c) Ataxia; PPt-W: - NE $(1,4)$; PPD: Tolerance and withdrawal, exacerbated by naltrexone. Some M-like signs missing; RI: SM - withdrawal, no weight loss. Previously reported - SDS: NE, 1981.

d) Previously reported - 1993.

e) Low potency $\kappa$-antagonist activity (and some $\delta$-antagonist activity).

f) Convulsions noted.

g) Low potency $\mu$-partial agonist.

h) Weak $\kappa$-antagonist.

i) Not $\mu$-opioid action; produces CNS excitation.

j) No opioid agonist action; $\mu$-antagonist (not simple competitive). 

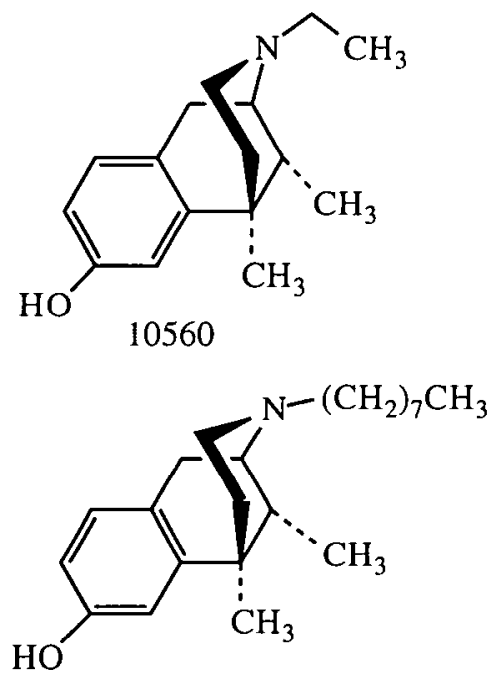

10697

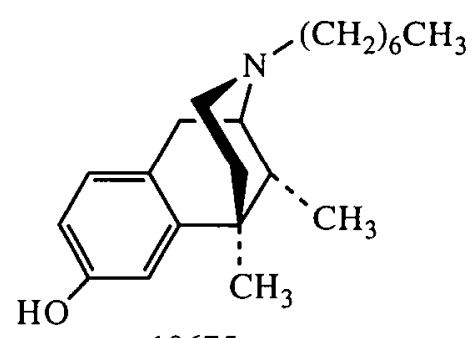

10675

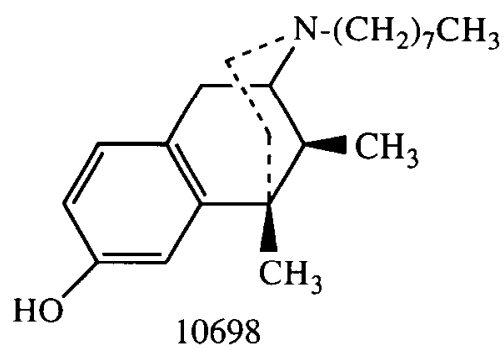

MOUSE ED50/AD50

IN VITRO

\begin{tabular}{|c|c|c|c|c|c|c|c|}
\hline NIH \# & $\mathrm{HP}$ & PPQ & $\mathrm{TF}$ & TFA & RBH & VD & SDS \\
\hline 10560 & $\mathrm{I}^{\mathrm{b}}$ & $1.4^{\mathrm{b}}$ & $\mathrm{I}^{\mathrm{b}}$ & $3.7^{b}$ & $144 \mathrm{nM}^{\mathrm{b}}$ & ANTAG $^{b, c}$ & $\mathrm{NS}^{\mathrm{d}}$ \\
\hline 10675 & $2.4^{\mathrm{e}}$ & $0.13^{\mathrm{f}}$ & $1.7^{\mathrm{f}}$ & I & $89 \mathrm{nM}$ & $3.5 \mathrm{E}-7(100)^{\mathrm{g}}$ & $\begin{array}{l}\mathrm{NS} \\
(1.25,5)^{\mathrm{h}}\end{array}$ \\
\hline 10697 & $5.4^{1}$ & $0.5^{1}$ & $10.0^{i}$ & $\overline{\mathrm{I}^{1}}$ & $226 \mathrm{nM}^{\mathrm{i}, \mathrm{j}}$ & $\mathrm{ANT}^{1, \mathrm{k}}$ & $\mathrm{NS}(1,4)^{1}$ \\
\hline 10698 & $11.1^{1}$ & $7.8^{1}$ & $\mathrm{I}^{1}$ & $\mathrm{I}^{1}$ & $3.4 \mu \mathrm{M}^{\mathrm{i}, \mathrm{m}}$ & $\mathrm{ANT}^{\mathrm{i}, \mathrm{n}}$ & $\mathrm{NS}(4,16)^{1}$ \\
\hline
\end{tabular}

a) See text for explanation of column headings and abbreviations.

b) Previously reported - 1988; TF: $\mathrm{pA}_{2}$ vs $\mathbf{M}: 5.85, \mu$-competitive.

c) Unusual, non-competitive, non-selective.

d) Exacerbates withdrawal.

f) Previously reported - 1992 .

g) Agonist at $\mu$ and $\kappa$ receptors.

h) Convulsions with cumulative dose of $11.5 \mathrm{mg} / \mathrm{kg} / \mathrm{hr}$; RI: SM - PS; RI: PPD - $\mu$-like withdrawal.

i) Previously reported - 1993.

j) BIND: $\mu=150, \delta:=197, \mathbf{K}:=115 \mathrm{nM}$ (non-selective).

k) Non-typical $\mu$ - and $\kappa$-agonist, weak $\mu$-antagonist.

1) May exacerbate withdrawal, possible $\kappa$-agonist properties.

m) BIND: $\mu=763 \mathrm{nM}, \delta==21 \%$ at $6 \mu \mathrm{M}, \kappa=2.8 \mu \mathrm{M}$ (non-selective, low potency)

n) Weak $\mu$ - and $\kappa$-antagonist. 
<smiles>CCC(=O)C1(c2cccc(O)c2)CCN(Cc2ccc([N+](=O)[O-])cc2)CC1</smiles>

10738<smiles>CCC(=O)C1(c2cccc(O)c2)CCN(CC=C(C)C)CC1</smiles><smiles>CCOC(=O)C1(c2cccc(O)c2)CCN(Cc2ccc(F)cc2)CC1</smiles>

10739 10795

MOUSE ED50/AD50

IN VITRO

MONKEY

\begin{tabular}{|l|l|l|l|l|l|l|l|}
\hline$\underline{\mathrm{NIH} \#}$ & $\underline{\mathrm{HP}}$ & $\underline{\mathrm{PPO}}$ & $\underline{\mathrm{TF}}$ & $\underline{\underline{\mathrm{AF}}}$ & $\underline{\mathrm{RBH}}$ & $\underline{\mathrm{VD}}$ & $\underline{\underline{\mathrm{SDS}}}$ \\
\hline & & & & & & & \\
\hline 10738 & $\mathrm{I}$ & 7.3 & $\mathrm{I}$ & $\mathrm{I}$ & $3.4 \mu \mathrm{M}^{\mathrm{b}}$ & $\mathrm{ANT}^{\mathrm{b}, \mathrm{c}}$ & $\mathrm{NS}(3,12)$ \\
\hline 10739 & $\mathrm{I}$ & 18.3 & $\mathrm{I}$ & $\mathrm{I}$ & $1.3 \mu \mathrm{Mb}$ & $\mathrm{ANT}^{\mathrm{b}, \mathrm{d}}$ & $\mathrm{NS}(2.5,10)$ \\
\hline 10795 & 8.3 & 2.2 & 9.7 & $\mathrm{I}$ & $507 \mathrm{nM}^{\mathrm{b}}$ & $\mathrm{ANT}^{\mathrm{b}, \mathrm{e}}$ & $\mathrm{PS}(2.5,10)^{\mathrm{f}}$ \\
\hline
\end{tabular}

a) See text for explanation of column headings and abbreviations.

b) Previously reported - 1993 .

c) Low potency $\kappa$ - and $\delta$-antagonist, not simple competitive-type.

d) Low potency, mostly $\delta$-antagonist (some $\kappa$-antagonism), not competitive.

e) Weak non-opioid partial agonist; weak non-selective antagonist.

f) Non-dose related suppression. Some observed signs seen from abrupt withdrawal are more typical from precipitated withdrawal. 
<smiles>CCO[C@H](CN1CC[C@@H](N(C(=O)CC)c2ccccc2)[C@H](C)C1)c1ccccc1</smiles><smiles>CCC(=O)N(c1ccccc1)[C@@H]1CCN(C(CO)c2ccccc2)C[C@H]1C</smiles>

10765<smiles>CCC(=O)N(c1ccccc1)[C@H]1CCN(CC(=O)c2cccs2)C[C@H]1C</smiles><smiles>CCC(=O)N(c1ccc(F)cc1)[C@@H]1CCN(C[C@H](O)c2ccccc2)C[C@H]1C</smiles>

MOUSE ED50/AD50

IN VITRO MONKEY

\begin{tabular}{|ll|l|l|l|l|l|l|}
\hline$\underline{\mathrm{NIH}}$ & $\underline{\mathrm{HP}}$ & $\underline{\mathrm{PPO}}$ & $\underline{\mathrm{TF}}$ & $\underline{\mathrm{FA}}$ & $\underline{\mathrm{RBH}}$ & $\underline{\mathrm{VD}}$ & $\underline{\underline{S D S}}$ \\
\hline 10741 & $1 \mathrm{E}-4^{\mathrm{b}}$ & $9 \mathrm{E}-5^{\mathrm{b}}$ & $2 \mathrm{E}-4^{\mathrm{b}, \mathrm{c}}$ & $\mathrm{I}^{\mathrm{b}}$ & $5.9 \mathrm{nM}^{\mathrm{b}}$ & $\begin{array}{l}56 \mathrm{fM} \\
{[\mathrm{A}]^{\mathrm{b}, \mathrm{d}}}\end{array}$ & $\begin{array}{l}\mathrm{CS}^{\mathrm{b}, \mathrm{e}}(1.5 \mathrm{E}- \\
4-3 \mathrm{E}-5)\end{array}$ \\
\hline 10762 & $0.24^{\mathrm{b}}$ & $0.02^{\mathrm{b}}$ & $0.03^{\mathrm{b}, \mathrm{f}}$ & $\mathrm{I}^{\mathrm{b}}$ & $569 \mathrm{nM}$ & $>3 \mu \mathrm{g}$ & $\mathrm{CS}[60 \times \mathrm{x}]^{\mathrm{b}}$ \\
\hline 10765 & $3.4^{\mathrm{b}}$ & $0.1^{\mathrm{b}}$ & $3.0^{\mathrm{b}}$ & $\mathrm{I}^{\mathrm{b}}$ & $6 \mu \mathrm{M}$ & $\mathrm{ANT}^{\mathrm{h}}$ & $\begin{array}{l}\mathrm{CS} \\
{[1.5 \times \mathrm{M}]^{\mathrm{b}}}\end{array}$ \\
\hline 10784 & 0.005 & 0.001 & $0.004^{\mathrm{l}}$ & $\mathrm{I}$ & $6.5 \mathrm{nM}^{\mathrm{b}}$ & $\begin{array}{l}7.6 \mathrm{nM} \\
{[\mathrm{A}]^{\mathrm{b}, \mathrm{j}}}\end{array}$ & $\begin{array}{l}\mathrm{CS} \\
{[6000 \times \mathrm{x}]}\end{array}$ \\
\hline 10785 & 0.001 & $3.4 \mathrm{E}-4$ & $0.002^{\mathrm{k}}$ & $\mathrm{I}$ & $4.5 \mathrm{nM}$ & $\begin{array}{l}2.8 \mathrm{nM} \\
(100)[\mathrm{A}]^{1}\end{array}$ & $\begin{array}{l}\mathrm{CS} \\
{[1000 \times \mathrm{x}]}\end{array}$ \\
\hline
\end{tabular}

a) See text for explanation of column headings and abbreviations.

b) Previously reported - 1993.

c) Naloxone $\left(\mathrm{AD}_{50}\right)$ vs $\mathrm{ED}_{80}$ of $10741=0.008 . \mathrm{pA}_{2}$ vs naloxone $=7.2$, suggesting competive interaction with $\mu$-receptor.

d) Biphasic (also, $4 \mathrm{nM}[\mathrm{A}])$. Complex actions $-\delta$-agonist activity.

e) $20,000-50,000 \times \mathrm{M}$.

f) Naloxone $\left(\mathrm{AD}_{50}\right)$ vs $\mathrm{ED}_{80}$ of $10762=0.03$.

g) Low potency $\mu$-opioid agonist.

h) Very low potency $\mu$-opioid antagonist.

i) Naloxone $\left(\mathrm{AD}_{50}\right)$ vs $\mathrm{ED}_{80}$ of $10784=0.11$.

j) Potent $\mu$-agonist.

k) Naloxone $\left(\mathrm{AD}_{50}\right)$ vs $\mathrm{ED}_{80}$ of $10785=0.04$.

1) $\delta$-Selective agonist. 

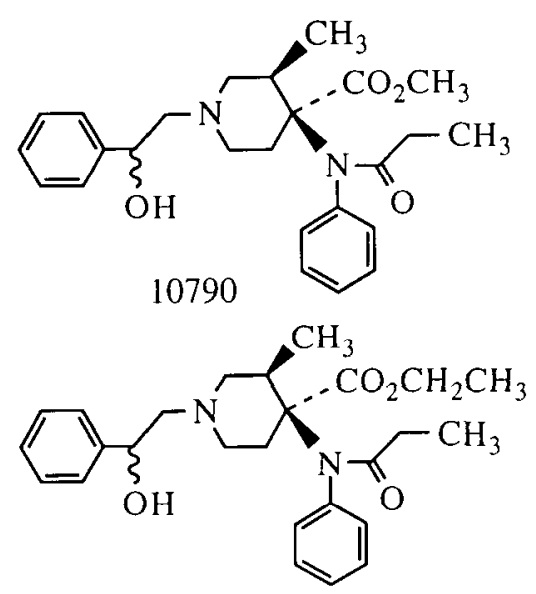

10792<smiles>CCC(=O)N(c1ccccc1)[C@]1(C(=O)OC)CCN(C[C@H](O)c2ccccc2)C[C@H]1C</smiles><smiles>CCC(=O)N(c1ccccc1)[C@]1(C)CCN(C[C@H](O)c2ccccc2)C[C@H]1C</smiles>

10793

MOUSE ED50/AD50

IN VITRO

MONKEY

\begin{tabular}{|l|l|l|l|l|l|l|l|}
\hline$\underline{\mathrm{NIH}} \#$ & $\underline{\mathrm{HP}}$ & $\underline{\mathrm{PPQ}}$ & $\underline{\mathrm{TF}}$ & $\underline{\mathrm{TFA}}$ & $\underline{\mathrm{RBH}}$ & $\underline{\mathrm{VD}}$ & $\underline{\mathrm{SDS}}$ \\
\hline & & & & & & & \\
\hline 10786 & 0.013 & 0.007 & $0.03^{\mathrm{b}}$ & $\mathrm{I}$ & $\begin{array}{l}22.9 \\
\mathrm{nM}\end{array}$ & $1.4 \mu \mathrm{M}^{\mathrm{c}}$ & CS $[60 \times \mathrm{x}]$ \\
\hline 10790 & $6.1 \mathrm{E}-4$ & $1 \mathrm{E}-4$ & $4.4 \mathrm{E}-4^{\mathrm{d}}$ & $\mathrm{I}$ & $\begin{array}{l}0.85 \\
\mathrm{nM}\end{array}$ & $0.77 \mathrm{nM}[\mathrm{A}]$ & $\begin{array}{l}\mathrm{CS} \\
{[6000 \times \mathrm{x}]}\end{array}$ \\
\hline 10791 & 0.001 & $8 \mathrm{E}-4$ & $0.001^{\mathrm{e}}$ & $\mathrm{I}$ & $2.3 \mathrm{nM}$ & $1.3 \mathrm{nM}[\mathrm{A}]^{\mathrm{f}}$ & $\begin{array}{l}\mathrm{CS} \\
{[6000 \times \mathrm{M}]}\end{array}$ \\
\hline 10792 & 0.003 & $1 \mathrm{E}-4$ & $2.4 \mathrm{E}-4^{\mathrm{g}}$ & $\mathrm{I}$ & $1.2 \mathrm{nM}$ & $1.3 \mathrm{nM}[\mathrm{A}]^{\mathrm{f}}$ & $\begin{array}{l}\mathrm{CS} \\
{[30,000 \times \mathrm{x}]}\end{array}$ \\
\hline 10793 & $3.1 \mathrm{E}-4$ & $5 \mathrm{E}-4$ & $0.0013^{\mathrm{h}}$ & $\mathrm{I}$ & $\begin{array}{l}0.78 \\
n M\end{array}$ & $0.84 \mathrm{nM}[\mathrm{A}]^{\mathrm{f}}$ & $\begin{array}{l}\mathrm{CS} \\
{[10,000 \times \mathrm{M}]}\end{array}$ \\
\hline
\end{tabular}

a) See text for explanation of column headings and abbreviations.

b) Naloxone $\left(\mathrm{AD}_{50}\right)$ vs $\mathrm{ED}_{80}$ of $10786=0.04$.

c) Complex, multiphasic action, weak antagonist

d) Naloxone $\left(\mathrm{AD}_{50}\right)$ vs $\mathrm{ED}_{80}$ of $10790=0.02 ; \mathrm{pA}_{2}$ naloxone vs $10790=7.4$.

e) Naloxone $\left(\mathrm{AD}_{50}\right)$ vs $\mathrm{ED}_{80}$ of $10791=0.02 ; \mathrm{pA}_{2}$ naloxone vs $10791=7.5$.

f) Potent, somewhat $\mu$-selective agonist.

g) Naloxone $\left(\mathrm{AD}_{50}\right)$ vs $\mathrm{ED}_{80}$ of $10792=0.04$.

h) Naloxone $\left(\mathrm{AD}_{50}\right)$ vs $\mathrm{ED}_{80}$ of $10793=0.04$. 
<smiles>CC(CN(C)C(=O)Cc1ccc(Cl)c(Cl)c1)N(C)C</smiles><smiles>CCCN1CCN(CCc2ccc(Cl)c(Cl)c2)CC1</smiles>

10782

MOUSE ED50/AD50

IN VITRO

MONKEY

\begin{tabular}{|l|l|l|l|l|l|l|l|}
\hline$\underline{\mathrm{NIH} \#}$ & $\underline{\mathrm{HP}}$ & $\underline{\text { PPQ }}$ & $\underline{\mathrm{TF}}$ & $\underline{\mathrm{TFA}}$ & $\underline{\mathrm{RBH}}$ & $\underline{\mathrm{VD}}$ & $\underline{\text { SDS }}$ \\
\hline & & & & & & & \\
\hline 10700 & $\mathrm{I}^{\mathrm{b}}$ & $0.3^{\mathrm{b}}$ & $4.8^{\mathrm{b}, \mathrm{c}}$ & $\mathrm{I}$ & $\begin{array}{l}619 \\
n \mathrm{M}^{\mathrm{d}, \mathrm{e}}\end{array}$ & $\mathrm{ANT}^{\mathrm{d}, \mathrm{f}}$ & $\mathrm{NT}$ \\
\hline 10703 & $\mathrm{I}^{\mathrm{d}}$ & $13.4^{\mathrm{d}}$ & $\mathrm{I}^{\mathrm{d}}$ & $\mathrm{I}^{\mathrm{d}}$ & $>6 \mu \mathrm{M}^{\mathrm{d}, \mathrm{g}}$ & $49 \mu \mathrm{M}(100)[\mathrm{A}]^{\mathrm{d}, \mathrm{h}}$ & $\mathrm{NS}^{\mathrm{d}, \mathrm{1}}$ \\
\hline 10705 & $\mathrm{I}^{\mathrm{d}}$ & $10.6^{\mathrm{d}}$ & $\mathrm{I}^{\mathrm{d}}$ & $\mathrm{I}^{\mathrm{d}}$ & $>6 \mu \mathrm{M}^{\mathrm{d}, \mathrm{j}}$ & $\mathrm{NE}^{\mathrm{d}}$ & $\mathrm{N} \mathrm{S}^{\mathrm{d}, \mathrm{k}}$ \\
\hline 10782 & $\mathrm{I}$ & $\mathrm{I}$ & $\mathrm{I}$ & $\mathrm{I}$ & $>6 \mu \mathrm{M}$ & $3.7 \mathrm{E}-8(30)[\mathrm{A}]^{\mathrm{l}}$ & $\mathrm{N} \mathrm{S}^{\mathrm{m}}$ \\
\hline
\end{tabular}

a) See text for explanation of column headings and abbreviations.

b) Previously reported - 1991 (potent $\sigma$-ligand).

c) Straub tail, ataxia, convulsions.

d) Previously reported - 1992 (potent $\sigma$-ligand).

e) BIND: $\mu=62 \mathrm{nM}, \delta=2370 \mathrm{nM}, \boldsymbol{\kappa}=2695 \mathrm{nM}$.

f) Weak, non-selective narcotic antagonist.

g) BIND: $\mu=1791 \mathrm{nM}, \delta:=>6 \mu \mathrm{M}, \kappa=287 \mathrm{nM}$.

h) Possibly some $\kappa$, or mixed $\mu-\kappa$ activity.

i) Disorientation noted.

j) BIND: $\mu=5210 \mathrm{nM}, \delta=>6 \mu \mathrm{M}, \kappa=3181 \mathrm{nM}$.

k) Exacerbates withdrawal signs.

1) Possible low potency agonist.

m) Cumulative dose of $11 \mathrm{mg} / \mathrm{kg}$. 

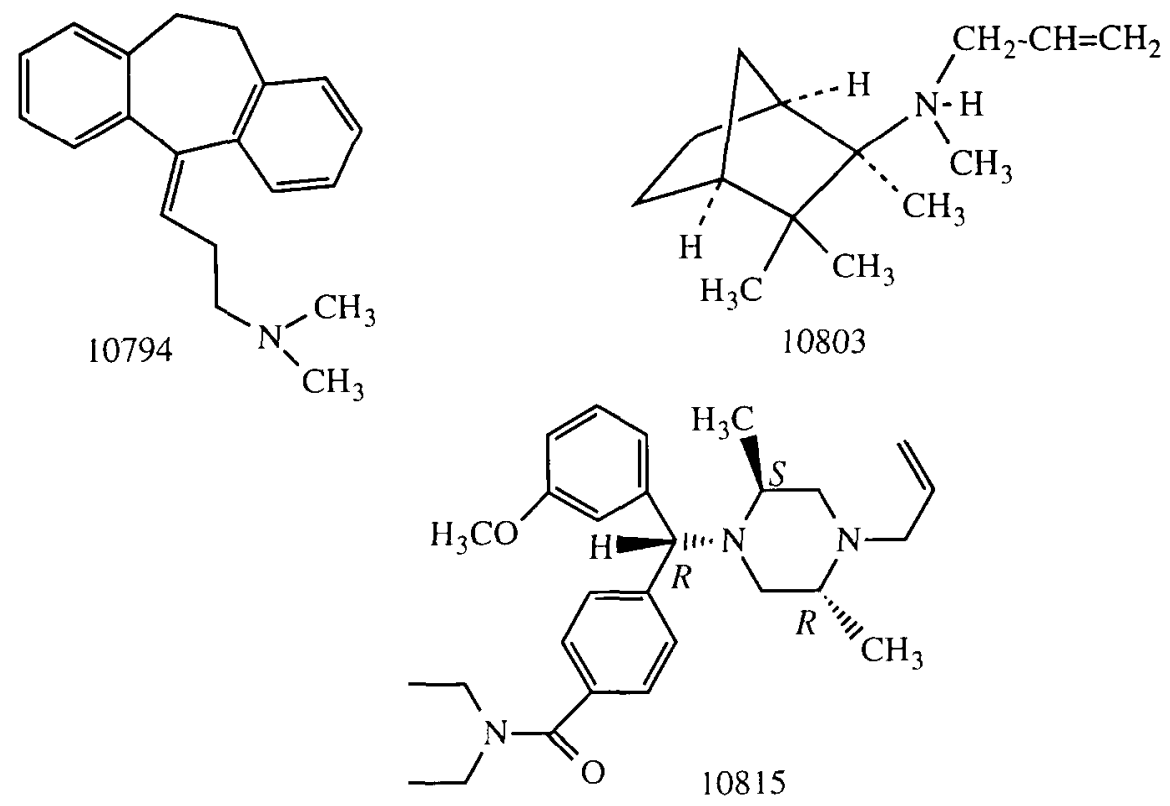

MOUSE ED50/AD50 IN VITRO MONKEY

\begin{tabular}{|l|l|l|l|l|l|l|l|}
\hline$\underline{\mathrm{NIH}} \#$ & $\underline{\mathrm{HP}}$ & $\underline{\mathrm{PPQ}}$ & TF & TFA & $\underline{\mathrm{RBH}}$ & $\mathrm{VD}$ & $\underline{\underline{S}}$ \\
\hline & & & & & & & \\
\hline 10794 & $\mathrm{I}$ & 0.09 & $\mathrm{I}$ & $\mathrm{I}$ & $>6 \mu \mathrm{M}$ & $3 \mathrm{E}-9(39)[\mathrm{A}]^{\mathrm{b}}$ & $\mathrm{CS}(2,8)[\mathrm{NA}]^{\mathrm{C}}$ \\
\hline 10803 & $\mathrm{I}$ & 9.8 & $\mathrm{I}$ & $\mathrm{I}$ & $>6 \mu \mathrm{M}$ & $1.8 \mathrm{E}-9(23)[\mathrm{NA}]^{\mathrm{d}}$ & $\mathrm{NS}^{\mathrm{e}}$ \\
\hline 10815 & - & - & - & - & $>6 \mu \mathrm{M}$ & $6.4 \mathrm{E}-9(100)[\mathrm{A}]^{\mathrm{I}}$ & - \\
\hline
\end{tabular}

a) See text for explanation of column headings and abbreviations.

b) Low efficacy $\mu$-agonist, low potency antagonist.

c) Drug actions not opioid-like. In mice, naloxone did not prevent nor antagonize drug actions. In non-dependent monkeys, the overt neurological signs associated with withdrawal were not antagonized by naloxone.

d) Naloxone decreased maximum response without shift in concentration-effect curve; weak partial agonist or non-opioid agonist.

e) Non- $\mu$-opioid properties.

f) Relatively selective for $\delta$-receptors. 
<smiles>O=C(CC1c2ccccc2C(=O)N1c1ccc2ccc(Cl)nc2n1)N1CCC2(CC1)OCCO2</smiles>

CPDD 0038

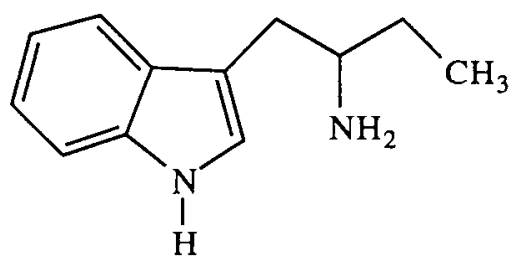

CPDD 0041

\begin{tabular}{|c|c|c|c|c|c|c|}
\hline CPDD\# & $\mathrm{SLA}^{\mathrm{a}}$ & $\underline{I S}^{b}$ & PD-S ${ }^{\mathrm{c}}$ & PD-PPD $^{\text {d }}$ & ${\underline{\mathrm{SA}^{\mathrm{e}}}}^{\mathrm{e}}$ & ${\underline{\mathrm{DD}^{\mathrm{f}}}}^{\mathrm{f}}$ \\
\hline 038 & Stumulant $\mathrm{g}^{\mathrm{g}}$ & Impaired $^{h}$ & NT & NT & Insoluble & $\mathrm{NO}^{\mathrm{i}}$ \\
\hline 941 & Stumulant & Impaired $\mathrm{d}^{\mathrm{j}}$ & $\mathrm{NT}$ & NT & $\mathrm{YES}^{\mathrm{k}}$ & $\mathrm{NO}^{1}$ \\
\hline
\end{tabular}

a) Spontaneous locomotor activity (mouse).

b) Inverted screen assay (mouse).

c) Physical dependence - substitution for pentobarbital (rat infusion).

d) Physical dependence - primary (rat infusion).

e) Self-administration (monkey).

f) Drug discrimination (intragastric administration, monkey).

g) Mild stimulation, not dose-related

h) Non-specific toxicity: impairment not dose-related. Drug actions not typical of usual stimulants or depressants.

i) Does not share discriminative stimulus effects with $d$-amphetamine or pentobarbital.

j) Stimulant efficacy and potency greater than cocaine and less than $d$ amphetamine.

k) Reinforcing effects in two out of three cocaine-trained monkeys. Unusually variable rates of responding.

1) Does not share discriminative stimulus effects with pentobarbital; may have slight, but not full discriminative stimulus effects with $d$-amphetamine. 


\section{REFERENCES}

(1) Fukuda, K.; Kato, S.; Mori, K.; Nishi, M.; Takeshima, H. Primary Structures and Expression from cDNAs of Rat Opioid Receptor $\delta$ - and $\mu$ Subtypes. FEBS Lett. 1993, 327, 311-314.

(2) Evans, C. J.; Keith, D. E.; Morrison, H.; Magendzo, K.; Edwards, R. H. Cloning of a Delta Opioid Receptor by Functional Expression. Science 1992, 258, 1952-1954.

(3) Kieffer, B. L.; Befort, K.; Gaveriaux-Ruff, C.; Hirth, C. G. The $\delta$-Opioid Receptor: Isolation of a cDNA by Expression Cloning and Pharmacological Characterization. Proc. Natl. Acad. Sci. USA 1992, 89, 12048-12052.

(4) Meng, F.; Xie, G. X.; Thompson, R. C.; Mansour, A.; Goldstein, A.; Watson, S. J.; Akil, H. Cloning and Pharmacological Characterization of a Rat K-Opioid Receptor. Proc. Natl. Acad. Sci. USA. 1993, 90, 9954-9958.

(5) Wang, J. B.; Imai, Y. S.; Eppler, C. M.; Gregor, P.; Spivak, C. E.; Uhl, G. R. $\mu$-Opiate Receptor: cDNA Cloning and Expression. Proc. Natl. Acad. Sci. USA. 1993, 90, 10230-10234.

(6) Chen, Y.; Mestek, A.; Liu, J.; Hurley, J. A.; Yu, L. Molecular Cloning and Functional Expression of a $\mu$-Opioid Receptor from Rat Brain. Mol. Pharmacol. 1993, 44, 8-12.

(7) Minami, M.; Toya, T.; Katao, Y.; Maekawa, K.; Nakamura, S.; Onogi, T.; Kaneko, S.; Satoh, M. Cloning and Expression of a cDNA for the Rat Kappa-Opioid Receptor. FEBS Lett. 1993, 329, 291-295.

(8) Nishi, M.; Takeshima, H.; Fukuda, K.; Kato, S.; Mori, K. cDNA Cloning and Pharmacological Characterization of an Opioid Receptor with High Affinities for Kappa-Subtype-Selective Ligands. FEBS Lett. 1993, 330, 77-80.

(9) Aceto, M. D.; Bowman, E. R.; Harris, L. S.; May, E. L. Dependence Studies of New Compounds in the Rhesus Monkey, Rat and Mouse (1994). In Problems of Drug Dependence 1994; L. S. Harris, Ed.; NIDA Research Monograph: Washington, D.C., 1995; pp in press.

(10) Woods, J. H.; France, C. P.; Medzihradsky, F.; Smith, C. B.; Winger, G. D. Evaluation of New Compounds for Opioid Activity. Annual Report (1994). In Problems of Drug Dependence 1994; L. S. Harris, Ed.; NIDA Research Monograph: Washington, D.C., 1995; pp in press.

(11) Jacobson, A. E. Biological Evaluation of Compounds for their Physical Dependence Potential and Abuse Liability. XVII. Drug Evaluation Committee of the College on Problems of Drug Dependence, Inc. (1993). In Problems of Drug Dependence 1994; L. S. Harris, Ed.; NIDA Research Monograph 140: Washington, D.C, 1994; Vol. I; pp 179-195.

(12) Aceto, M. D.; Bowman, E. R.; Harris, L. S.; May, E. L. Dependence Studies of New Compounds in the Rhesus Monkey, Rat and Mouse (1993). In Problems of Drug Dependence 1993; L. S. Harris, Ed.; NIDA Research Monograph 140: Washington, D.C., 1994; Vol. I; pp 103-178. 
(13) Woods, J. H.; France, C. P.; Medzihradsky, F.; Smith, C. B.; Winger, G. D. Evaluation of New Compounds for Opioid Activity. Annual Report (1993). In Problems of Drug Dependence 1993; L. S. Harris, Ed.; NIDA Research Monograph 140: Washington, D.C., 1994; Vol. I; pp 196-247.

(14) Patrick, G. A.; Harris, L. S.; Woolverton, W. L.; Nader, M. A.; Winger, G.; Woods, J. H. Progress Report From the Testing Program for Stimulant and Depressant Drugs (1993). In Problems of Drug Dependence 1993; L. S. Harris, Ed.; NIDA Research Monograph 140: Washington, D.C., 1994; Vol. I; pp 248-263.

(15) May, E. L.; Aceto, M. D.; Bowman, E. R.; Bentley, C.; Martin, B. R.; Harris, L. S.; Medzihradsky, F.; Mattson, M. V.; Jacobson, A. E. Antipodal $\alpha$-N-Alkyl (Methyl-Decyl)-N-Normetazocines (5,9 $\alpha$-Dimethyl2'-hydroxy-6,7-benzomorphans): In Vitro and In Vivo Properties. J. Med. Chem. 1994, in review.

(16) Winger, G.; Woods, J. H.; Patrick, G. A.; Harris, L. S.; Woolverton, W. L. Progress Report From the Testing Program for Stimulant and Depressant Drugs. In Problems of Drug Dependence 1994 ; L. S. Harris, Ed.; NIDA Research Monograph: Washington, D.C., 1995; pp in press.

(17) Jacobson, A. E. Biological Evaluation of Compounds for their Physical Dependence Potential and Abuse Liability. IX. Drug Testing Program of the Committee on Problems of Drug Dependence, Inc. (1985). NIDA. Res. Monogr. 1986, 67, 385-398.

\section{AFFILIATION}

A. E. Jacobson, Ph.D., Biological Coordinator, Drug Evaluation Committee, CPDD; Laboratory of Medicinal Chemistry, National Institute of Diabetes and Digestive and Kidney Diseases, National Institutes of Health, Bethesda, Maryland 20892. 


\title{
PROGRESS REPORT FROM THE TESTING PROGRAM FOR STIMULANT AND DEPRESSANT DRUGS (1994)
}

\author{
G. Winger; W. L. Woolverton; J. K. Rowlett; J. A. English; G. A. Patrick; \\ M. A. Nader; R. E. McDaniel; W. T. Hawkins; B. W. Massey; L. S. Harris; \\ and J. H. Woods
}

The research group involved in the evaluation of stimulant and depressant compounds has been in existence for approximately ten years. The group includes laboratories at Virginia Commonwealth University (Patrick, Harris, McDaniel, Hawkins), University of Mississippi Medical Center (Woolverton, Rowlett, English; earlier work at the University of Chicago was assisted by Nader and Massey)), the University of Michigan (Winger, Woods) and NIH (Dr. Aurthur E. Jacobson). The group is part of the Drug Evaluation Committee, chaired by Dr. Ted Cicero, of the College on Problems of Drug Dependence (CPDD) and is supported by both CPDD and NIDA. One of the purposes of the group is to evalute new compounds, generally classified as either stimulants or depressants, for their abuse liability and potential to produce dependence. Compounds are received, coded and distributed by Dr. Jacobson for blind testing in the various laboratories. They are evaluated for discriminative stimulus effect (UMS), reinforcing effects (UM) and capacity to produce physical dependence (VCU). This report includes the results of evaluation of the following compounds: CPDD-0038 (2-(7-Chloro-1,8-naphthyridin-2-yl)-3-[(1,4-dioxa-8azaspiro[4,5]dec-8-yl)carbonylmethyl]isoindolin-1-one); CPDD-0041 (etryptamine acetate: $\alpha$-ethyl-1H-indole-3-ethanamine acetate).

\section{METHODS}

\section{Reinforcing Effects in Rhesus Monkeys}

The reinforcing effects of CPDD-0041 were evaluated in a substutution selfadministration procedure with cocaine serving as the baseline drug. Rhesus monkeys were surgically prepared with indwelling silicone rubber catheters using $10 \mathrm{mg} / \mathrm{kg}$ i.m. ketamine and $2.0 \mathrm{mg} / \mathrm{kg}$ i.m. xylazine as anesthetics. Catheters were implanted in jugular (internal or external), femoral or brachial veins as necessary. The catheter passed subcutaneously from the site of the incision to the mid-scapular region, where it exited the monkey and continued through a 
hollow restraining arm to the outside rear of the cage.

The restraint and catheter protection device has been described in detail by Deneau et al. (1969). Monkeys were individually housed in stainless steel cages, measuring 83.3 X 76.2 X $91.4 \mathrm{~cm}$ deep. Each monkey wore a tubular stainless steel harness that protected the exit site of the catheter and allowed relatively unrestricted movements within the cage. A Teflon cloth jacket (Alice King Chatham Medical Arts, Los Angeles, CA) provided further protection for animals who tended to locate and pull their catheters. The harness was connected to a flexible spring arm that carried the catheter to the back of the cage where it joined tubing passing through a roller infusion pump (Watson and Marlow Co., Model MHRK 55, Falmouth, UK).

A $16.4 \mathrm{~cm}$ square stimulus panel was located on the side of each cage, approximately $10 \mathrm{~cm}$ from the front and $19 \mathrm{~cm}$ from the bottom of the cage. Across the top of the stimulus panel, $2.5 \mathrm{~cm}$ apart, were three circles, $2.5 \mathrm{~cm}$ in diameter, covered with translucent plastic and capable of being illuminated from behind by $5 \mathrm{~W}$ colored bulbs. The two side lights could be illuminated red and the center light green. Below each of the two red stimulus lights was a response lever (Model 121-07; BRS-LVE, Beltsville, MD) capable of being operated by 10-15 gm of force. Experimental control was provided by an IBM PS/2 computer programmed with Med-PC (Med-Associates, Fairfield, VT) software and located in an adjoining room.

The drug self-administration procedure has been described in detail, in Winger et al. (1989). There were two daily sessions of drug availability each day: each session was $130 \mathrm{~min}$ in duration. Sessions were signalled by the onset of a red stimulus light over one of the levers. Each session was divided into four components, and a different dose of cocaine was available during each component. Approximately every third session, saline, rather than a cocaine solution, was delivered for the entire session. Each component was 25 min or 20 injections in duration and was followed by a 10 min timeout period, during which all lights were turned off in the cage and responding had no programmed consequences. The schedule of drug delivery during each component was a fixed-ratio 30 time-out $45 \mathrm{sec}$. Thirty responses on the lever beneath the light turned off this light and turned on the infusion pump and the green light. Each infusion was followed by a $45 \mathrm{sec}$ period when all lights were extinguished and responses had no programmed consequences. At the end of this time period, the red stimulus light was again illuminated, signalling drug availability.

Changes in cocaine dose were accomplished by altering the duration of the infusion pump. The durations were $0.50,1.7,5.0$, and $16.7 \mathrm{sec}$, and the drug concentration, based on the weight of monkey, provided for infusion doses of $0.001,0.003,0.01$, and $0.03 \mathrm{mg} / \mathrm{kg} / \mathrm{inj}$. The same procedure was used to evaluate CPDD-0041, but on different sessions the drug concentration was altered so that a wide range of doses were evaluated. The infusion durations during a single session were always the same: $0.5,1.7,5.0$ and $16.7 \mathrm{sec}$. 
The order of drug dose was varied randomly among four orders on each session. One of the dose orders was ascending, one was descending and two were mixed. Earlier data with cocaine have shown that dose order did not modify greatly the response rates maintained by an individual dose (Winger et al., 1989). This may not be true of long-acting drugs,

\section{Discriminative Stimulus Effects in Rhesus Monkeys}

The subjects were adult rhesus monkeys weighing between 6.5 and $13 \mathrm{~kg}$. All monkeys had extensive experience with the present drug discrimination procedure. They were housed individually in stainless steel cages in which water was continuously available. They were fed 100 to $150 \mathrm{~g}$ of monkey chow after each session and were given a chewable vitamin tablet 3 days/week. During experimental sessions each monkey was seated in a Plas-Lab restraining chair and placed in a wooden cubicle (175 $\mathrm{cm}$ high X $85 \mathrm{~cm}$ wide $X 65 \mathrm{~cm}$ deep) containing two response levers mounted $100 \mathrm{~cm}$ above the floor. A $40 \mathrm{~W}$ white house light was mounted on the ceiling. The monkey's feet were placed into shoes, the bottoms of which were fitted with brass plates which could deliver electric shocks. Programming and recording of experimental events were accomplished by a computer (Aim 65 microprocessor or Macintosh II) located in an adjacent room.

The monkeys had been trained previously to discriminate d-amphetamine (AMPH) or pentobarbital (PB) from saline in a two-lever, discrete-trail shock avoidance procedure similar to the one described by Holtzman (1982). One hour after an intragastric infusion (via nasogastric tube) of the training drug ( 0.56 or $1.0 \mathrm{mg} / \mathrm{kg}$ AMPH or $10 \mathrm{mg} / \mathrm{kg}$ PB) or saline, the houselights and lever lights were illuminated (trial) and responding on one lever (designated the correct lever) avoided electric shock and extinguished the lights. Responding on the incorrect lever started a 2-second changeover delay during which correct lever responding had no consequence. If a correct lever response was not made within 5 seconds of onset of the lights, an electric shock $(250 \mathrm{msec}$ duration, $7 \mathrm{~mA}$ intensity) was delivered; if a correct response was made within $2 \mathrm{sec}$ after the first shock (escape), the trial was terminated, otherwise a second shock automatically ended a trial. Two consecutive trials with escape failure automatically ended the session. Trials were separated by a 30 -sec TO. The session lasted 30 trials or $20 \mathrm{~min}$, whichever came first. The correct lever was determined by the infusion that was administered before the session. For three monkeys, the right lever was correct after drug infusion and the left lever was correct after saline infusions. This condition was reversed for the other three monkeys.

Monkeys were considered to be stable in the discrimination when more than $90 \%$ of the trials were avoidance trials and the first response in the session was on the correct lever on at least seven out of eight consecutive sessions. At this point, testing was begun. Two 5-day sequences alternated drug, vehicle and test sessions so that the first test session was preceded by two training sessions, one 
with saline and one with drug pretreatment and the second test session of the sequence was preceded by either vehicle or drug pretreatment. In the event that the criterion for stimulus control was not met during the training sessions, the training sequence was continued. During test sessions, both levers were operational, i.e., shock could be avoided by responding on either lever.

Saline and at least three doses of each test drug were evaluated under the test conditions for each monkey. The percentage of trials that were completed on the drug lever is presented for each test session. In addition, the average time between the onset of a trial and a lever press (average latency) was calculated for each test session. Because these test compounds were evaluated blind without any potency information, initial test doses were done in an ascending order from $0.1 \mathrm{mg} / \mathrm{kg}$ to doses that either significantly increased latency to respond or resulted in at least $90 \%$ drug-appropriate responding. Out of concern for the monkeys, doses greater than $30 \mathrm{mg} / \mathrm{kg}$ were not tested. If a dose substituted for a training drug, that dose and doses higher and lower were tested again, in a random order.

A stock solution of AMPH was dissolved in saline to a final concentration of 5 $\mathrm{mg} / \mathrm{ml}$. Sodium pentobarbital was diluted with $0.9 \%$ saline to a final concentration of $40 \mathrm{mg} / \mathrm{ml}$, from a stock solution of $400 \mathrm{mg} / \mathrm{ml}$ that contained propylene glycol, 95\% EtOH and water in a ratio of 4:1:5. CPDD-0038 was dissolved in acetic acid immediately before administration. CPDD-0041 was dissolved in saline. Doses of CPDD-0038 were tested twice in PB-trained monkeys and once in AMPH-trained monkeys. Doses of CPDD-0041 were tested twice in AMPH-trained monkeys and once in PB-trained monkeys.

\section{Physical Dependence Studies in Rats and Potency Estimates in Mice}

Male Sprague-Dawley rats (Harlan Labs, Dublin, VA) initially weighing 200-225 $\mathrm{g}$ were individually housed in stainless steel cages with food and water freely available. They were used in the chronic infusion and substitution experiments. CF-1 mice (Harlan Labs, Dublin, VA) weighing 25 to $35 \mathrm{~g}$ were housed in plastic cages with food and water ad lib. The mice were used in initial studies of potency estimation. All animals were acclimated to the animal facility for several days prior to use in any study.

Rats were surgically prepared with an intraperitoneal cannula (PE90 tubing) while under methoxytlurane anesthesia. Acclimation to the infusion system occurred for at least two days during which the rats were infused with $0.9 \%$ saline. This was followed by the continuous infusion of either saline (control), pentobarbital sodium or cocaine hydrochloride for 12 consecutive days using an escalating dosing schedule (Yutrzenka et al., 1985). At the end of the infusion period most rats were receiving pentobarbital at a dose of $850-900 \mathrm{mg} / \mathrm{kg} / 24 \mathrm{hr}$ or cocaine at a dose of $160-170 \mathrm{mg} / \mathrm{kg} / 24 \mathrm{hr}$. Body weight was monitored daily during the drug infusion period. 
Following the final day of pentobarbital or cocaine infusion, a 24-hour substitution period commenced during which the rats were infused with either saline, vehicle, or test drug. This was followed by a 24-hour drug withdrawal period during which all rats received saline.

Every two hours for the first 12 hours and again at 24 hours of each period, rats were assigned a withdrawal score based on the degree of expression of several behavioral responses and signs. In addition, body weight was determined at 0 , 8 and 24 hours of each period. Scores were assigned by two observers who were blind to the drug treatment. Investigators were blind to the identity of the compounds until all data were collected and analyzed (Yutrzenka et al., 1989).

Preliminary studies to ascertain potency of the test compounds were conducted in mice. Drug-treated mice were assayed using the inverted screen test (Coughenour et al, 1977) and alteration of spontaneous locomotor activity. At least three doses of each drug, with at least six mice per dose, were used to determine dose-response curves. Vehicle-treated mice served as controls and were assayed concurrently with drug-treated mice.

Each mouse was placed on a wire screen $(12.8 \mathrm{X} 12.8 \mathrm{~cm}$ with a $6 \mathrm{~mm}$ mesh) and the screen was then inverted. A positive drug effect was to cause the mouse to fall from the screen within 60 seconds. A negative effect was scored if the mouse climbed to the top of the screen within $60 \mathrm{sec}$. Hanging from the underside of the screen was recorded as a one-half positive effect (Coughenour et al., 1977). The inverted screen test was conducted at 30, 60, 120 and $240 \mathrm{~min}$ following drug administration.

Spontaneous locomotor activity was determined using a 16-beam infrared photocell (Omintech, Columbus, $\mathrm{OH}$ ) which transected a plastic cage containing one mouse. Movement of the mice disrupted the beam(s) and a "count" of activity was recorded. Following drug administration, activity was recorded at 5-15 $\mathrm{min}, 35-50 \mathrm{~min}, 65-95 \mathrm{~min}$, and 125-185 $\mathrm{min}$. The ED50 dose for a depressant drug was determined to be that dose which reduced spontaneous locomotor activity to one-half that recorded for concurrently tested vehicletreated control mice. For stimulant drugs, activity at a given dose was expressed as "percent of control activity". Potency estimates of each test drug were determined at time of peak activity and when, in addition, the vehicle effect was no longer evident.

Pentobarbital sodium was dissolved in distilled water made isotonic with sodium chloride. CPDD-0038 was suspended in a vehicle including Tween 80 (20\%) propylene glycol $(20 \%)$ and 1 drop of acetic acid in distilled water to make 10 $\mathrm{ml}$. CPDD-004 1 was dissolved in isotonic saline. The CPDD-0038 suspension and the CPDD-0041 solution or vehicle were given by i.p. injection in a volume of $10 \mathrm{mg} / \mathrm{ml}$.

Withdrawal scores for each treatment group were compared to the control by use 
of the Mann-Whitney U-test. Alterations in body weight were tested for significance by use of the t-test. ED50 values and 95\% confidence intervals in the inverted screen test aned locomotor activity measure were also determined when appropriate by the method of Litchfield and Wilcoxon (1949).

CPDD-0038

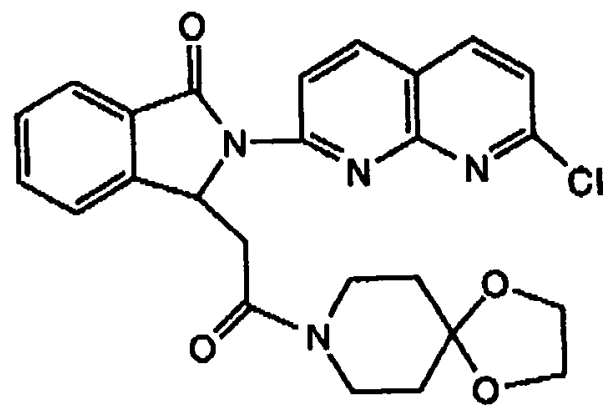

\section{Reinforcing Effects in Rhesus Monkeys}

Due to limited solubility, CPDD-0038 could not be evaluated in tests of intravenous reinforcing effects in monkeys.

\section{Drug Discrimination in Studies in Rhesus Monkeys}

CPDD-0038 occasioned primarily saline-appropriate responding in all monkeys up to $30 \mathrm{mg} / \mathrm{kg}$. Latency was not systematically affected. Some monkeys vomited at $30 \mathrm{mg} / \mathrm{kg}$.

\section{Potency Estimation in Mice}

The effects of CPDD-0038 on spontaneous locomotor activity were generally unremarkable. A mild stimulant effect seemed most prevalent, but it was neither profound nor clearly dose-related. At the highest dose $(50 \mathrm{mg} / \mathrm{kg})$ the mice exhibited Straub tail and were jumping uncontrollably in their cages within approximately $10 \mathrm{~min}$ after injection of drug. 
CPDD-0038 (continued)

Table 1: Effects of CPDD-0038 on Spontaneous Locomotor Activity

\begin{tabular}{|l|l|l|l|l|}
\hline \multicolumn{5}{|c|}{ Time after Treatment (min) } \\
\hline \hline Dose $(\mathrm{mg} / \mathrm{kg})$ & $5-15$ & $35-50$ & $65-95$ & $125-185$ \\
\hline \hline 10 & $68^{\mathrm{a}}$ & 120 & 159 & 120 \\
\hline 20 & 106 & 52 & 77 & 56 \\
\hline 40 & 119 & 158 & $1190^{*}$ & 116 \\
\hline
\end{tabular}

${ }^{\text {a }}$ Values expressed as percent of control activity of concomitantly tested, vehicle-treated mice.

* Control mice exhibited unusually low activity, and one cage of test mice exhibited very high activity compared to other treated pairs.

\section{Inverted Screen Test}

The lower doses of CPDD-0038 (10 and $20 \mathrm{mg}$ ) produced effects ranging from $0 \%$ to $50 \%$ impairment in the performance of this task, but those effects were not clearly time nor dose-related. The $40 \mathrm{mg} / \mathrm{kg}$ dose produced an effect greater than $80 \%$ impairment across the time course up to $120 \mathrm{~min}$ after injection.

Summary. CPDD-0038 does not fit the profile or either a typical stimulant or depressant drug; it does not have PB- or AMPH-like discriminative effects.

CPDD-0041

Etryptamine acetate ( $\alpha$-ethyl-1H-indole-3-ethanamine acetate, or $\alpha$-ethyltryptamine acetate)<smiles>CCC(N)Cc1c[nH]c2ccccc12</smiles>

\section{Reinforcing Effects in Rhesus Monkeys.}

The reinforcing effects of CPDD-0041 ranging from 0.00001 to $1.0 \mathrm{mg} / \mathrm{kg} / \mathrm{inj}$ were evaluated in three monkeys. In monkey JA, none of these doses 


\section{CPDD-0041 (continued)}

maintained rates of responding indicative of a reinforcing effect. In monkeys DA and HE, doses of 0.03 and $0.3 \mathrm{mg} / \mathrm{kg} / \mathrm{inj}$ respectively maintained rates indicative of a reinforcing effect. Rates maintained by these doses varied considerably from one exposure to the next for no apparent reason. Monkey $\mathrm{HE}$, on one occasion, received a dose order that included $3.2 \mathrm{mg} / \mathrm{kg} / \mathrm{inj}$. He self-administered approximately $25 \mathrm{mg} / \mathrm{kg}$ of CPDD-41 during this session, and was markedly affected following the session. He had dilated pupils and was tremoring and appeared to be pre-convulsive. One $\mathrm{mg} / \mathrm{kg}$ diazepam was administered, and the monkey appeared to be much calmer. Figure 1 shows the results of the self-administration study.

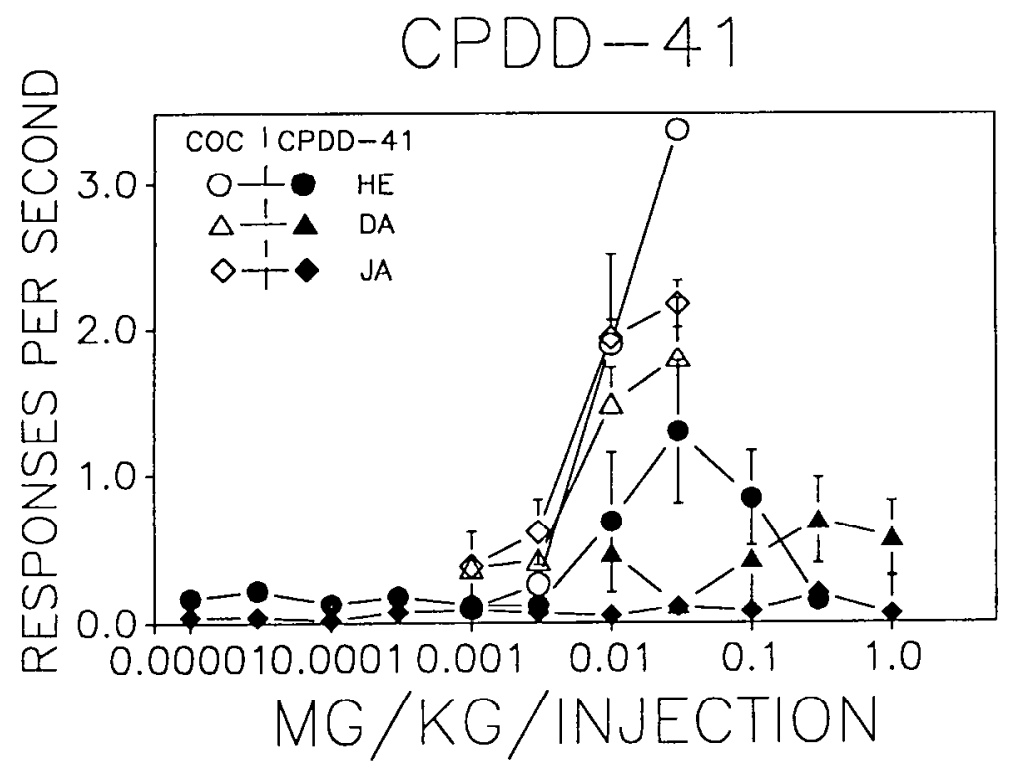

Figure 1. Self-administration of CPDD-0041 in three rhesus monkeys. The open symbols are rates of responding maintained by cocaine in these animals. The different symbols represent data from individual monkeys, as indicated by the legend.

\section{Drug Discrimination Studies in Rhesus Monkeys}

CPDD-0041 engendered a maximum of $63.5 \%$ drug lever responding in one AMPH-trained monkey at $17 \mathrm{mg} / \mathrm{kg}$ (8906; Table 2). There was little or no drug lever responding up to $10 \mathrm{mg} / \mathrm{kg}$ CPDD-0041 in the other two monkeys. Although $31 \%$ of the trials were completed by 7739 on the drug lever at 3.0 $\mathrm{mg} / \mathrm{kg}$, this was the result of averaging one test session with $93 \%$ of the trials completed on the drug lever with two test sessions with no trials completed on the drug lever. No trials were completed on the drug lever when the dose was increased to 10 and $17 \mathrm{mg} / \mathrm{kg}$, suggesting that the partial substitution in 


\section{CPDD-0041 (continued)}

7739 at $3.0 \mathrm{mg} / \mathrm{kg}$ probably involved "noise" rather than an actual AMPH-like effect. Latency to respond increased with dose of CPDD-0041 in all monkeys.

CPDD-0041 engendered essentially exclusive saline lever responding in all three PB - trained monkeys. All monkeys ate preferred foor offered immediately after the session at $10 \mathrm{mg} / \mathrm{kg}$ CPDD-0041. As with AMPHtrained monkeys, latency to respond was not systematically affected by CPDD-0041.

Table 2: Discriminative stimulus effects of intragastric administration of CPDD-0041 in AMPH- or pentobarbital-trained monkeys

\begin{tabular}{|c|c|c|c|c|c|c|}
\hline \multicolumn{7}{|c|}{ CPDD-0041 (mg/kg) } \\
\hline $\begin{array}{l}\text { AMPH- } \\
\text { trained } \\
\text { subject }\end{array}$ & Saline & 0.03 & 1 & 3 & 10 & 17 \\
\hline 7739 & $\begin{array}{l}0 / \\
0.9\end{array}$ & & $\begin{array}{l}0 / \\
1.0\end{array}$ & $\begin{array}{l}31 / \\
0.98\end{array}$ & $\begin{array}{l}0 / \\
1.08\end{array}$ & $\begin{array}{l}0 / \\
1.2\end{array}$ \\
\hline 8906 & $\begin{array}{l}0 / \\
0.88\end{array}$ & & $\begin{array}{l}26.5 / \\
1.0\end{array}$ & $\begin{array}{l}18 / \\
1.25\end{array}$ & $\begin{array}{c}63.5 / \\
1.28\end{array}$ & $\mathrm{nt}$ \\
\hline 8515 & $\begin{array}{l}0 / \\
0.5\end{array}$ & & $\mathrm{nt}$ & $\begin{array}{l}0 / \\
0.52\end{array}$ & $\begin{array}{l}0 / \\
0.62\end{array}$ & $\begin{array}{l}0 / \\
0.06\end{array}$ \\
\hline $\begin{array}{l}\text { PB- } \\
\text { trained } \\
\text { subject }\end{array}$ & & & & & & \%.\%. \\
\hline 7976 & $\begin{array}{l}0 / \\
0.99\end{array}$ & $\begin{array}{l}0 / \\
1.0\end{array}$ & $\begin{array}{l}0 / \\
0.9\end{array}$ & n.t. & $\begin{array}{l}0 / \\
1.0\end{array}$ & \\
\hline 8814 & $\begin{array}{l}0 / \\
0.85\end{array}$ & $\begin{array}{l}0 / \\
0.77\end{array}$ & $\begin{array}{l}0 / \\
0.78\end{array}$ & $\begin{array}{l}3 / \\
0.87\end{array}$ & $\begin{array}{l}10 / \\
0.63\end{array}$ & \\
\hline 8236 & $\begin{array}{l}0 / \\
0.93\end{array}$ & $\begin{array}{l}0 / \\
0.77\end{array}$ & \begin{tabular}{|l|}
$0 /$ \\
1.61
\end{tabular} & $\begin{array}{l}0 / \\
0.87\end{array}$ & $\begin{array}{l}0 / \\
1.1\end{array}$ & \\
\hline
\end{tabular}

Rhesus monkeys were trained to discriminate either $0.561 .0 \mathrm{mg} / \mathrm{kg}$ (i.g.) AMPH or $10.0 \mathrm{mg} / \mathrm{kg}$ (i.g.) PB from saline in a discrete-trials avoidance-escape paradigm Data presented represent the percent drug-appropriate responding/average response latency (sec). CPDD-0041 was administered via nasogastric tube 60 min prior to testing. In all cases 30 trials were completed.

$\mathrm{nt}=$ not tested 


\section{Potency Estimation in Mice}

The compound caused a mild increase in activity at 2.0 to $5.0 \mathrm{mg} / \mathrm{kg}$, and the effect became significant at $10.0 \mathrm{mg} / \mathrm{kg}$ (Table 3 ). The stimulant effect was dose-related, although the increase in activity with each increment in disage was rather mild, and there was no appreciable difference between doses of 15 and $30 \mathrm{mg} / \mathrm{kg}$. One notable aspect of the stimulant effect was that it seemed rather slow in onset and prolonged in duration, with the last time interval measured (125 to $185 \mathrm{~min}$ after treament) corresponding to the peak effect. At the dose of $15.0 \mathrm{mg} / \mathrm{kg}$ there was a significant impairment of performance in the inverted screen task, with an $88 \%$ effect at 30 min that rapidly declined to $25 \%$ effect at $60 \mathrm{~min}$ post-treatment. At the $30.0 \mathrm{mg} / \mathrm{kg}$ dose all mice were rendered unable to perform the task at $30 \mathrm{~min}$, and a $75 \%$ effect persisted for $240 \mathrm{~min}$, probably indicative of some nonspecific toxicity at this dosage.

Table 3: Effects of CPDD-0041 on Spontaneous Locomotor Activity

\begin{tabular}{|l|l|l|l|l|}
\hline \multicolumn{5}{|c|}{ Time after Treatment (min) } \\
\hline \hline $\begin{array}{l}\text { Dose } \\
(\mathrm{mg} / \mathrm{kg})\end{array}$ & $5-15$ & $35-50$ & $65-95$ & $125-185$ \\
\hline \hline 2 & $196^{\mathrm{a}}$ & 110 & 79 & 218 \\
\hline 5 & 114 & 136 & 116 & 155 \\
\hline 10 & 120 & 259 & 294 & 473 \\
\hline 15 & 116 & 304 & 442 & 741 \\
\hline 30 & 184 & 244 & 301 & 933 \\
\hline
\end{tabular}

${ }^{\text {a }}$ Values expressed as percent of control activity have concomitantly tested, vehicle-treated mice.

\section{Infusion in Pentobarbital-Dependent Rats}

When administered to rats undergoing withdrawal from pentobarbital, CPDD0041 caused a significant enhancement in both overt signs of withdrawal (Figure 2) when compared with saline substitution. However, these effects were no greater in pentobarbital-abstinent rats than in drug-naive rats that were given CPDD-0041 following 12 days of infusion of saline, so the effect does not appear to be additive with barbiturate withdrawal. 


\section{Substitution in Rats Chronically Infused with Cocaine}

When CPDD-004 1 was infused in rats that had received cocaine for 12 days, it produced suppression of food intake during the $24 \mathrm{hr}$ in which it was substituted. It also caused a significant loss in body weight, which persisted for at least $24 \mathrm{hr}$ after the drug itself was discontinued.

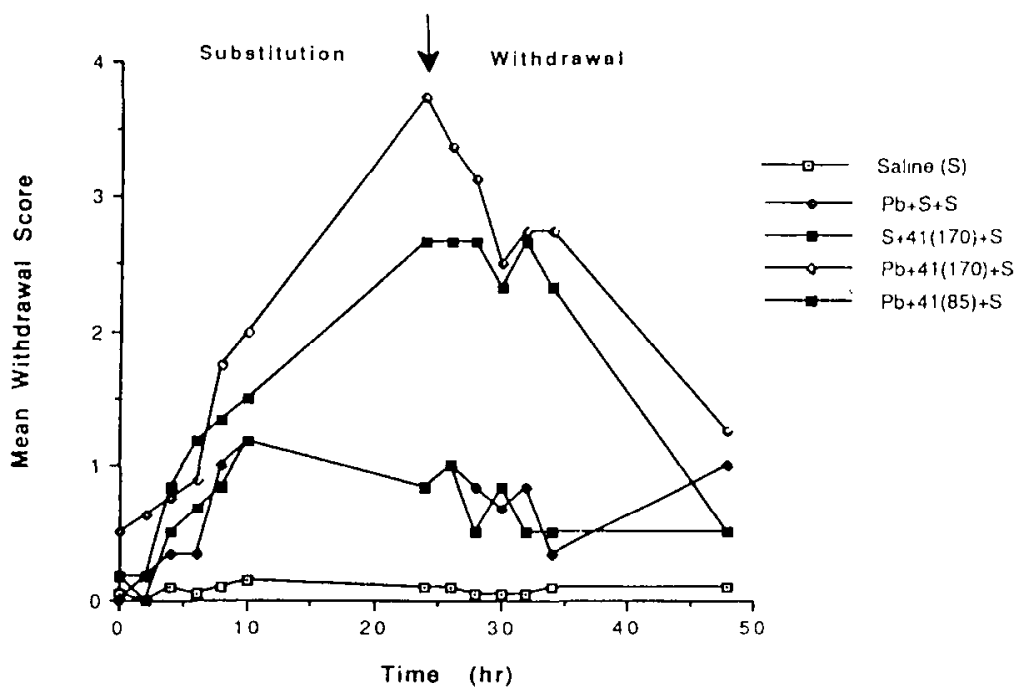

Figure 2. Mean withdrawal scores of control rats on $\mathrm{PB}$ - dependent rats during substitution and subsequent withdrawal of CPDD-0041.

Summary. The general profile of activity of etryptamine is more stimulant than depressant. The stimulant activity was not profound, however. Etryptamine produced less locomotor stimulation than did AMPH in mice, it produced a partial AMPH-like discriminative stimulus effect in only one monkey, and was not self-administered at all by the third monkey.

\section{References}

Coughenour, L.L.; and McLean, J.R. A new device for the rapid measurement of impaired motor function in mice. Pharmacol Biochem Behav 6:351-353, 1977.

Deneau, G.A.; Yanagita, T.; and Seevers, M.H. Self-administration of 
psychoactive substances by the monkey. A measure of psychological dependence. Psychopharmacologia 16:30-48, 1969.

Litchfield and Wilcoxon, 1949

Winger, G.D.; Palmer, R.K.; and Woods, J.H. Drug-reinforced responding: rapid determination of dose response functions. Drug Alc Depend 24:135-142, 1989.

Yutrzenka, G.J.; Patrick, G.A.; and Rosenberger, W. Continuous intraperitoneal infusion of pentobarbital: a model of barbiturate dependence in the rat. J Pharmacol Exp Ther 232: 111-118, 1985.

Yutrzenka, G.J.; Patrick, G.; and Rosenberger, W. Substitution of temazepam and midazolam in pentobarbital-dependent rats. Physiol Behav 46:55-60.

AFFILIATION: Departments of Psychology and Pharmacology, University of Michigan, Ann Arbor, MI; Department of Psychiatry, and Pharmacology and Toxicology, University of Mississippi Medical Center, Jackson, MS; Department of Pharmacology and Toxicology, Virginia Commonwealth University/Medical College of Virginia, Richmond, VA. 


\title{
EVALUATION OF NEW COMPOUNDS FOR OPIOID ACTIVITY 1994
}

\author{
J. H. Woods, F. Medizhradsky, C. B. Smith, C. P. France and \\ C. Winger
}

This report contains information on opioid abuse liability evaluations on compounds that have been submitted to the Drug Evaluation Committee of the College and released for publication by the submitters. The information obtained can involve both in vitro evaluation in opioid binding assays and smooth muscle (largely, mouse vas deferens preparations. In addition, the compounds may be evaluated for discriminative and reinforcing effects. Analgesic and respiratory function assays arc also possible. These behavioral assessments are conducted in rhesus monkeys. Each of these assays is described below. Usually when limited information is provided (e.g., in vitro assessment only), it is because the sample provided by the submitter was insufficient to carry out further evaluation.

The evaluation of new compounds by the programs at the University of Michigan and the Medical College of Virginia is coordinated by Dr. Arthur E. Jacobson, Laboratory of Medicinal Chemistry, NIDDK, National Institutes of Health, Bethesda, MD. The compounds, which come originally from pharmaceutical companies. universities, government laboratories, and international organizations are submitted to Dr. Jacobson.

At the UM and MCV laboratories, drug samples arrive from Dr. Jacobson with only the following information: (1) an identifying NIH number, (2) molecular weight, (3) solubility information and (4) a recommended starting dose. After the evaluation is complete and the report submitted to Dr. Jacobson, the submitter is requested to release the chemical structure to include with the evaluation data in the ANNUAL REPORT. The submitter has up to three years before release of the structure is required. When the structure is released all of the data on the compound are reported to the Drug Evaluation Committee.

\section{DRUG DISCRIMINATION IN RHESUS MONKEYS}

We currently use three groups of monkeys to test the discriminative stimulus effects of submitted drugs: one of these groups discriminates the administration of the $\kappa$ agonist ethylketazocine (EKC); a second group discriminates the $\mu$ agonist alfentanil; a third group is treated daily with morphine and discriminates the opioid antagonist naltrexone.

The procedures used with the EKC-trained monkeys have been described by Bertalmio et al., (1982). The monkeys are removed from their home cages each day and seated in primate restraining chairs. These chairs arc placed in isolation chambers equipped with two response levers, several stimulus lights and a cup to receive Noyes, banana-flavored pellets. These monkeys are required to make 100 consecutive responses on the correct one of the two levers and receive ten $300-\mathrm{mg}$ food pellets. The right lever is correct if they 
were given a subcutaneous injection of $0.0032 \mathrm{mg} / \mathrm{kg}$ EKC immediately prior to the start of the cycle. The left lever is designated correct if they were given a sham injection before the start of the cycle. Each cycle lasts 15-min and consists of an initial 10-min black out period followed by a period of as long as $5 \mathrm{~min}$, during which a blue light is illuminated in the chamber and the monkey can respond for food. If the food pellets are delivered before the $5 \mathrm{~min}$ period is completed, the lights are extinguished for the remainder of this time. Typically, a daily session consists of several 15 min cycles. During a training session, if EKC is given, it is given on the penultimate cycle of that session. Responding on the drug-appropriate lever is reinforced during that cycle and on the subsequent, final cycle of the day. These last two cycles may be preceded by from zero to four sham cycles on a training day. A training session of six sham cycles is also scheduled from time to time.

With this type of multiple, discrete-cycle training, the animals can be tested with a cumulative dosing procedure. On a test session, the first cycle is preceded by an injection of saline, and prior to subsequent cycles, increasing, cumulative doses of the test drug are administered. One hundred consecutive responses on either lever are reinforced throughout the test session. The test drug is administered in increasing doses until the monkey either responds on the drug-appropriate lever, the response rate falls to less than half of the salinecontrol rate, or six cycles are given. In the latter situation, it is assumed that the selected dose range is too low, and the test is continued at higher doses on the next test session. Each test session is preceded and followed by a training session. The criterion for satisfactory performance must be met on each training session that is followed by a test session. This criterion is that at least $90 \%$ of the responses during each cycle of a training session must be on the injection-appropriate lever, either sham or EKC.

The procedure for the alfentanil-trained monkeys is similar, but not identical. These animals are also trained and tested in a discrete, multiple-cycle procedure. The main difference between the alfentanil procedure and the EKC procedure is that the alfentanil monkeys are required to make 20 rather than 100 responses, and they receive a single pellet for correct responses. They can receive as many as 10 pellets during the 5 -min, food-availability period of each cycle, but each pellet is delivered after 20 responses. Because in this procedure, monkeys can switch from one lever to another following the delivery of food, an additional criterion is added for satisfactory performance. In addition to making $90 \%$ or more of their responses on the correct lever, the monkeys must make fewer than 20 responses on the incorrect lever prior to delivery of the first food pellet of each cycle. Tests of the discriminative stimulus effects of submitted drugs in the alfentanil-trained monkeys are also done using a cumulative dosing procedure with dosing criteria identical to those used in the EKC-trained monkeys.

The procedure for studying discriminative stimulus effects in morphine-treated monkeys has been described previously (France and Woods, 1989). Daily sessions are comprised of a 10-min time out during which lever presses have no programmed consequence and a 5-min response period during which green stimulus lights are illuminated and signal the activation of a schedule of stimulus-shock termination. sessions consist of between two and six discrete, 15-min cycles with each cycle. Under these experimental conditions electric shock is scheduled to be delivered to the subject's feet every 15 seconds; monkeys can terminate the lights and postpone scheduled shocks for 30 
seconds by pressing five times consecutively (i.e., fixed-ratio 5) the lever appropriate for the solution administered during the first minute of the time out (left lever, saline; right lever, naltrexone). Monkeys receive an injection of saline $(0.1 \mathrm{ml} / \mathrm{kg})$ or drug $(0.01 \mathrm{mg} / \mathrm{kg}$ naltrexone $)$ during the first minute of each time out. On drug training days a single injection of naltrexone is administered during one time out and for that cycle and all subsequent cycles on that day only responding on the right lever postpones shocks. A variable number of saline cycles (0-5) precede the naltrexone cycle and on some days saline is administered during the time out of all cycles. Under these conditions monkeys switch their response choice from the saline lever to the naltrexone lever with complete generalization occurring in all three subjects at a dose of $0.01 \mathrm{mg} / \mathrm{kg}$. Responding on the naltrexone lever is accompanied by other behavioral effects indicative of opioid withdrawal (e.g., irritability, miosis, salivation). Moreover, when saline is substituted for the daily injection of 3.2 $\mathrm{mg} / \mathrm{kg}$ of morphine monkeys respond predominantly on the naltrexone lever and show directly observable signs of withdrawal; the discriminative stimulus and other effects produced by morphine abstinence are reversed by some opioid agonists (e.g., alfentanil; France and Woods, 1989; France et al., 1990).

For test sessions increasing doses of drug arc administered during the first minute of consecutive time outs and five consecutive responses on either lever postpone shocks. In monkeys that receive $3.2 \mathrm{mg} / \mathrm{kg}$ of morphine 3 hours earlier, increasing doses of a test compound are administered up to doses that produce an average of at least $80 \%$ responding on the naltrexone lever or to doses that disrupt responding and result in the delivery of electric shock. Drugs that do not substitute for nultrexone (i.e., precipitate withdrawal) are also studied for their ability to reverse responding on the naltrexone lever in morphine-abstinent (i.e., withdrawn) subjects. Test compounds are studied using a cumulative-dosing procedure in morphine-abstinent monkeys up to doses that reverse completely responding on the naltrexone lever $(<20 \%)$ or to doses that disrupt responding. Some compounds that substitute for naltrexone also are studied for their capacity to prevent the effects of cumulative doses of opioid agonists. Monkeys that receive saline three hours earlier, rather than the daily injection of morphine, receive saline (control) or a single injection of test compound during the first cycle and increasing doses of agonist (alfentanil or morphine) during subsequent cycles. Agonists are administered up to doses that produce a switch from the naltrexone lever to the saline lever or to doses that disrupt responding and result in the delivery of electric shock.

\section{THERMAL ANALGESIA IN RHESUS MONKEYS}

The tail withdrawal procedure used to study analgesic effects of test compounds in rhesus monkeys has been described previously (Dykstra and Woods, 1986). Monkeys are restrained loosely at the neck and arms while seated in Plexiglas primate chairs. For tests of tail withdrawal latency, the lower $10-12 \mathrm{~cm}$ of the shaved tail is immersed in a thermos containing water at $40^{\circ}, 50^{\circ}$, or $55^{\circ} \mathrm{C}$ and the latency until the tail is withdrawn from the thermos is recorded for each monkey at each temperature. When the tail is not withdrawn within 20 seconds (cut-off latency) the experimenter removes the thermos and a latency of 20 seconds is recorded. Experimental sessions begin with several exposures to $40^{\circ} \mathrm{C}$ water. Four or five monkeys are tested consecutively and the time between tail immersions for individual monkeys is 5 minutes. Generally, $40^{\circ} \mathrm{C}$ water does not produce tail withdrawal in rhesus 
monkeys (Dykstra and Woods, 1986); however, if a monkey fails to keep its tail in $40^{\circ} \mathrm{C}$ water for 20 seconds on at least 3 of 4 immersions, that animal is not tested further for that particular session. In a subsequent pre-test component, tails are immersed in $40^{\circ}, 50^{\circ}$, and $55^{\circ} \mathrm{C}$ water. The order in which the three temperatures are presented is varied among subjects. If the latencies for tail withdrawal in the pre-test component are at or near 20 seconds for $40^{\circ} \mathrm{C}$ water and less than 5 seconds for $55^{\circ} \mathrm{C}$ water, monkeys receive the test compound. The test is identical to the pre-test, except that monkeys receive s.c. injections of drug 10 minutes prior to tail immersion. The time between immersions for individual subjects is 5 minutes or less and the order in which temperatures are presented varies among subjects and across cycles. The inter-injection interval typically is 30 minutes and between four and six doses are studied in a single experiment using the cumulative dosing procedure. For some studies a single dose of an opioid antagonist is administered prior to the test compound and for other studies a single dose of test compound is administered prior to increasing doses of a $\mu$ (e.g., alfentanil)

or K (e.g., U-50,488) opioid agonist.

\section{RESPIRATORY STUDIES IN RHESUS MONKEYS}

The effects of test compounds on ventilatory function are studied in rhesus monkeys breathing air or 5\% $\mathrm{CO}_{2}$ in air (France and Woods, 1990; Howell et al., 1988). Monkeys are restrained at the neck and waist while seated in a Plexiglas primate chair. Normal air or $5 \% \mathrm{CO}_{2}$ in air is delivered at a rate of $101 / \mathrm{mm}$ into a sealed helmet placed over the subject's head. Changes in pressure within the helmet are measured and recorded by a transducer and a microprocessor, and are transformed according to known standards to frequency of respiration (f) in breaths/minute and to tidal volume (VT) in $\mathrm{ml} /$ inspiration. Data are recorded continuously during 23-minute exposures to air alternating with 7-minute exposures to $\mathrm{CO}_{2}$. The last three minutes of exposure to $\mathrm{CO}_{2}$ are used for data analyses and are compared to the last three minutes of exposure to air only. Increasing doses of drug are administered during the first minute of consecutive time outs so that the inter-injection interval is 30 minutes. For some studies a single injection of an opioid antagonist is administered prior to increasing doses of a test compound and for other studies a single injection of test compound is administered prior to cumulative doses of a standard compound (e.g., alfentanil).

\section{SELF-ADMINISTRATION BY MONKEYS}

Tests of self-administration determine the ability of the drug to maintain responding in monkeys trained to self-inject codeine. Each of at least three monkeys is studied with saline as a negative control and a number of doses of the test compound until a maximum rate of responding was obtained or until, in the absence of evidence of a reinforcing effect, observable changes in behavior are produced by the compound.

The schedule of intravenous drug delivery is a fixed-ratio 30; when a light above a lever is illuminated, the 30th response produces an intravenous drug injection accompanied by another light that is illuminated during drug delivery. After each injection, a $45 \mathrm{sec}$ timeout period occurs. A component of the session ends after 20 injections have been received or $25 \mathrm{~min}$ have passed, 
whichever occurs first. Different doses of the drug are available during each of four components of a session. Other procedural details are given in Winger et al., (1989).

\section{DISPLACEMENT OF RADIOLABELED LIGAND BINDING}

Details of the binding assay based on the displacement of ${ }^{3} \mathrm{H}$-etorphine in rat brain membranes have been described previously (Medzihradsky et al., 1984). Briefly, aliquots of a membrane preparation from rat cerebrum are incubated with ${ }^{3} \mathrm{H}$-etorphine in the presence of $150 \mathrm{mM} \mathrm{NaCl}$, and in the presence of different concentrations of the drug under investigation. Specific, i.e., opioidreceptor-related interaction of ${ }^{3} \mathrm{H}$-etorphine is determined as the difference in binding obtained in the absence and presence of an appropriate excess of unlabeled etorphine. The potency of the drugs in displacing the specific binding of ${ }^{3} \mathrm{H}$-etorphine is determined from log-probit plots of the data. See Table I for representative results with different opioids.

To enhance the characterization of novel opioids, we are also investigating their selectivity in binding to $\mu-, \partial-$, and $\kappa$-opioid receptors in membranes from monkey brain cortex. Thus, we are now providing $\mathrm{K}_{\mathrm{i}}$ values of the tested compounds in displacing the following radiolabeled opioid ligands:

etorphine (nonselective, reflects opioid character), sufentanil or Tyr-D-Ala-Gly-(Me)Phe-Gly-ol (DAMGO); ( $\mu$ selective), [D-Pen ${ }^{2}-\mathrm{D}-\mathrm{Pen}^{5}$ ] enkephalin (DPDPE, $\partial$ selective),

\section{U-69.593 ( $\kappa$ selective).}

Using the receptor-specific assays, we have described the selectivity of various established opioids in brain membranes of different species (Clark et al., 1988). The selection of monkey brain as the tissue for the selective binding assays strengthens the correlation between this in vitro assessment and the behavioral evaluation of the tested compounds. In the ANNUAL REPORT, the results of the selective binding assays are listed under "Binding in monkey brain cortex". See Table II for representative results with different opioids in rat and monkey brain. 


\section{TABLE I}

$\mathrm{EC}_{50}$ 's of representative opioids for displacement of $0.5 \mathrm{nM}{ }^{3} \mathrm{H}$-etorphine from rat brain membrane, and inhibition of the twitch of the mouse vas deferens preparation.

\begin{tabular}{lcc}
\hline Compound & $\begin{array}{c}\text { BINDING* }^{*} \\
\mathrm{EC}_{50}(\mathrm{nM})\end{array}$ & MVD \\
\hline DPDPE & --- & 5.52 \\
U50,488 & --- & 6.29 \\
Fentanyl & 36.2 & 37.1 \\
DAMGO & 23.9 & 81.3 \\
Etorphine & 0.37 & 0.0068 \\
(-)Cyclazocine & 0.53 & 11.9 \\
Naltrexone & 0.63 & --- \\
Bremazocine & 1.42 & 0.29 \\
UM 1071R** & 1.55 & --- \\
Sufentanil & 1.60 & 4.43 \\
(-)SKF 10047 & 3.93 & --- \\
Ethylketazocine & 6.60 & 11.6 \\
Ketazocine & 14.1 & 1.18 \\
Morphine & 23.6 & 395 \\
DSLET & 43.0 & 1.71 \\
Dextrorphan & $<6000$ & \\
& & \\
\hline
\end{tabular}

* In the presence of $150 \mathrm{mM} \mathrm{NaCl}$.

** 1R-5R-9R-2"R-5,9-dimethyl-2'-hydroxy-2-tetrahydrofurfuryl-6,7-benzomorphan hydrochloride 


\section{TABLE II}

Inhibition of radiolabeled sufentanil, DPDPE and U69,593 binding in rat and monkey brain. In membranes from rat cerebrum and monkey brain cortex, the inhibition of specific equilibrium binding of $0.5 \mathrm{nM}\left[{ }^{3} \mathrm{H}\right]$ sufentanil, $1.5 \mathrm{nM}\left[{ }^{3} \mathrm{H}\right]$ DPDPE and $1.5 \mathrm{nM}\left[{ }^{3} \mathrm{H}\right] \mathrm{U} 69,593$ by five different concentrations of the listed compounds was investigated in the presence of $150 \mathrm{mM} \mathrm{NaCl}$ (modified from Clark et al., 1988).

\begin{tabular}{|c|c|c|}
\hline Compound & $\begin{array}{cc} & \mathrm{EC}_{50}(\mathrm{nM}) \\
{\left[{ }^{3} \mathrm{H}\right] \text { Sufentanil }} & \left.{ }^{3} \mathrm{H}\right] \mathrm{DPDPE}\end{array}$ & {$\left[{ }^{3} \mathrm{H}\right] \mathrm{U} 69,593$} \\
\hline
\end{tabular}

\section{Rat cerebrum}

DAMGO

Sufentanil

Morphine

$\beta$-FNA

$\beta$-CNA

Naloxone

Etorphine

Buprenorphine

Bremazocine

Superfit

DSLET*

ICI- 174,864

DPDPE

$\mathrm{U} 50,488$

$\mathrm{U} 69,593$

\section{2}

1.25

31.4

6.99

1.29

6.37

0.60

1.07

1.79

576

121

58900

7720

7230

38000
690

45.0

422

43.9

7.48

14.3

1.13

1.12

1.12

16.5

1.05

59.0

6.44

13100

13400

\section{Monkey cortex}

$\begin{array}{lr}\text { Sufentanil } & 1.18 \\ \text { DPDPE } & 18900 \\ \text { U69,593 } & 10700\end{array}$

$\begin{array}{cr}81.1 & >10000 \\ 4.21 & >10000 \\ 7000 & 8.41\end{array}$

$\left(\mathrm{D}-\mathrm{Ser}^{2}, \mathrm{Leu}^{5}\right)$-enkephalin-Thr ${ }^{6}$ 


\section{ISOLATED, ELECTRICALLY-STIMULATED MOUSE VAS DEFERENS PREPARATION}

The development of new, highly selective antagonists such as the reversible $\boldsymbol{k}$ receptor antagonist norbinaltorphimine (Smith et al., 1989) and the competitive $\delta$ receptor antagonist ICI-174864 have made possible the evaluation of selectivity of opioid agonists and antagonists by use of the mouse vas deferens preparation. Male, albino ICR mice, weighing between 25 and $30 \mathrm{~g}$, are used. The mice are decapitated, the vasa deferentia removed, and $1.5 \mathrm{~cm}$ segments are suspended in organ baths which contain $30 \mathrm{ml}$ of a modified Kreb's physiological buffer. The buffer contains the following $(\mathrm{mM})$ : $\mathrm{NaCl}, 118 ; \mathrm{KCl}, 4.75 ; \mathrm{CaCl}_{2}, 2.54 ; \mathrm{MgSO}_{4}$, 1.19; $\mathrm{KH}_{2} \mathrm{PO}_{4}, 1.19$; glucose, 11; $\mathrm{NaHCO}_{3}, 25$; pargyline $\mathrm{HCl}, 0.3$; and disodium edetate, 0.03 . The buffer is saturated with $95 \% \mathrm{O}_{2}-5 \% \mathrm{CO}_{2}$ and kept at $37^{\circ} \mathrm{C}$. The segments are attached to strain gauge transducers and suspended between two platinum electrodes. After a 30-min equilibration period, the segments are stimulated once every $10 \mathrm{sec}$ with pairs of pulses of $2 \mathrm{msec}$ duration, $1 \mathrm{msec}$ apart and at supramaximal voltage. See Table III for potencies of representative agonists.

The following antagonists are studied: naltrexone HCl, ICI- 174864 [N,N-diallylTyr-Aib-Aib-Phe-Leu-OH] and norbinaltorphimine. The antagonists are added to the organ baths 15 minutes before the determination of cumulative concentration-effect relationships for the various agonists. See Table III for the potencies of different competitive antagonists studied in relation to prototypic agonists, $\mathrm{EC}_{50}$ 's are calculated by probit analysis, and $\mathrm{pA}_{2}$ values are determined to assess relative potencies of antagonists.

All drugs which are submitted for evaluation are studied in the following manner:

1) the submitted drug is tested on the vas deferens preparation in the absence and in the presence of a concentration of naltrexone sufficient to block $\mu, \mathbf{k}$ and $\delta$ receptors, 2) If the submitted drug inhibits the twitch and its actions are blocked by naltrexone, it is evaluated further in the absence and presence of ICI-174864 and norbinaltorphimine used in concentrations at which these antagonists are selective for $\delta$ and $\kappa$ receptors, respectively. 3) If the submitted drug is a partial agonist or devoid of agonistic activity at opioid receptors, it is evaluated further as an antagonist against the following agonists: sufentanil ( $\mu$ selective), DSLET ( $\delta$ selective) and U50,488 ( $\kappa$ selective). If the submitted drug has antagonistic activity against any or all of the receptor-selective agonists or upon any of the other preparations used in the Drug Evaluation Unit, the type of antagonism (competitive, noncompetitive, irreversible) is determined. For further details of the procedure and for a description of experiments in which $\mu$-funaltrexamine was used see Smith (1986). Drugs studied in the preparation prior to 1987 were evaluated with the protocol reported in the 1985 Annual Report. 


\section{TABLE III}

Potencies of antagonists assessed in the mouse vas deferens

$\mathrm{pA}_{2}$ values* determined with three agonists

Sufentanil $(\mu) \quad$ U50,488 $\left(\kappa^{\prime}\right) \quad \operatorname{DSLET}(\delta)$

\begin{tabular}{lrrr}
\hline Antagonist & & & \\
& & & \\
Naltrexone & 8.76 & 7.74 & 7.41 \\
Naloxone & 7.99 & 6.90 & 7.35 \\
Cyprodime* & 7.41 & 6.15 & 5.98 \\
Nalbuphine & 7.23 & 6.31 & 5.76 \\
Naltrindole & 7.71 & 7.38 & 9.44 \\
ICI- 174,864 & $<5.00$ & $<5.00$ & 7.90
\end{tabular}

*The $\mathrm{pA}_{2}$ value is the negative logarithm of the molar concentration of antagonist necessary to shift the agonist concentration-effect curve to the right by a factor of 2 -fold. 


\section{SUMMARY OF TESTS PERFORMED}

The compounds which were evaluated at the University of Michigan during the past year, and the individual tests which were performed are shown in Table IV. Also shown are dates of Reports to the Biological Coordinator, Dr. A.E. Jacobson, in which results are reported.

TABLE IV

SUMMARY OF TESTS PERFORMED

\begin{tabular}{|lc|c|c|c|c|c|c|}
\hline \multicolumn{1}{|l|}{$\mathrm{NIH \#}$} & $\mathrm{SA}$ & $\mathrm{MVD}$ & $\mathrm{BIND}$ & $\mathrm{DD}$ & $\mathrm{ANLG}$ & $\mathrm{RSP}$ & REPORT* \\
\hline 10683 & + & + & + & + & + & + & $02 / 03 / 92$ \\
\hline 10684 & + & + & + & - & + & + & $02 / 10 / 92$, \\
\hline 10697 & - & - & $\mathrm{MBC}$ & - & - & - & $06 / 28 / 93$ \\
\hline 10698 & - & - & $\mathrm{MBC}$ & - & - & - & $06 / 28 / 93$ \\
\hline 10700 & - & - & $\mathrm{MBC}$ & - & - & - & $06 / 28 / 93$ \\
\hline 10701 & - & - & $\mathrm{MBC}$ & - & - & - & $06 / 28 / 93$ \\
\hline 10702 & - & - & $\mathrm{MBC}$ & - & - & - & $06 / 28 / 93$ \\
\hline 10703 & - & - & $\mathrm{MBC}$ & - & - & - & $06 / 28 / 93$ \\
\hline 10705 & - & - & $\mathrm{MBC}$ & - & - & - & $06 / 28 / 93$ \\
\hline 10738 & - & + & + & - & - & - & $03 / 25 / 92$ \\
\hline 10739 & - & + & + & - & - & - & $03 / 25 / 92$ \\
\hline 10762 & - & + & + & - & - & - & $05 / 07 / 93$ \\
\hline 10765 & - & + & + & - & - & - & $04 / 23 / 93$ \\
\hline 10773 & - & + & + & - & - & - & $10 / 30 / 92$ \\
\hline 10782 & - & + & + & - & - & - & $03 / 05 / 93$ \\
\hline 10785 & - & + & + & - & - & - & $05 / 03 / 93$ \\
\hline 10786 & + & + & - & - & - & $05 / 07 / 93$ \\
\hline 10793 & - & + & + & - & - & $05 / 07 / 93$ \\
\hline
\end{tabular}


Table IV (continued)

\begin{tabular}{||l|c|c|c|c|c|c|c||}
\hline \hline $\mathrm{NIH}$ & $\mathrm{SA}$ & $\mathrm{MVD}$ & $\mathrm{BIND}$ & $\mathrm{DD}$ & ANGL & RSP & REPORT* \\
\hline 10787 & & + & + & - & - & - & $05 / 07 / 93$ \\
\hline 10790 & - & + & + & - & - & - & $02 / 20 / 94$ \\
\hline 10791 & - & + & + & - & - & - & $02 / 02 / 94$ \\
\hline 10792 & - & + & + & - & - & - & $02 / 02 / 94$ \\
\hline 10793 & - & + & + & - & - & - & $02 / 14 / 94$ \\
\hline 10802 & - & + & + & - & - & - & $02 / 14 / 94$ \\
\hline 10803 & - & + & + & - & - & - & $02 / 14 / 94$ \\
\hline 10805 & - & + & + & - & - & - & $02 / 12 / 93$ \\
\hline 10809 & - & + & + & - & - & - & $08 / 03 / 92$ \\
\hline 10810 & - & + & + & - & - & - & $08 / 03 / 92$ \\
\hline 10811 & - & + & + & - & - & - & $08 / 03 / 92$ \\
\hline 10812 & - & + & + & - & - & - & $02 / 02 / 92$ \\
\hline 10813 & - & + & + & - & - & - & $10 / 30 / 92$ \\
\hline 10814 & - & + & + & - & - & - & $11 / 12 / 93$ \\
\hline 10815 & & + & + & - & - & - & $03 / 09 / 93$ \\
\hline \hline
\end{tabular}

* Date report was submitted to CPDD Biological Coordinator. MBC $=$ Monkey Brain Cortex 
14-Hydroxy-N-(2-methoxyethyl)-7,8-dihydronormorphine

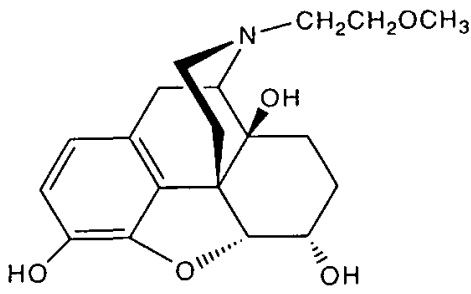

\section{DISPLACEMENT OF $\left[{ }^{3}\right.$ H]ETORPHINE BINDING}

$\mathrm{EC}_{50}$ of $8.24 \mathrm{nM}$ in the presence of 150 $\mathrm{mM} \mathrm{NaCI}$.

MOUSE VAS DEFERENS PREPARATION

\begin{tabular}{||l|l|l|l|l||}
\hline Condition & $\mathrm{EC}_{50}(\mathrm{nM})$ & $\begin{array}{l}\text { Maximum } \\
\text { Response (\%) }\end{array}$ & shift & $\mathrm{n}$ \\
\hline \hline Control & $7048.9 \pm 1499.6$ & 100 & & 9 \\
\hline Naltrexone $(100 \mathrm{nM})$ & $6468.9 \pm 1276.7$ & 100 & 0.9 & 3 \\
\hline ICI $174864(100 \mathrm{nM})$ & $5898.3 \pm 2685.4$ & 100 & 0.8 & 3 \\
\hline Nor-BNI $(10 \mathrm{nM})$ & $15226.0 \pm 9136.5$ & 100 & 2.2 & 3 \\
\hline \hline
\end{tabular}

\begin{tabular}{||l|l|l|l|c||}
\hline Agonist & $\mathrm{pA}_{2}$ & Slope \pm S.D. & $\begin{array}{l}\mathrm{pA2} \text { (Constrained) } \\
\pm \text { S.E. }\end{array}$ & $\mathrm{n}$ \\
\hline \hline Sufentanil & 7.35 & $1.01 \pm 0.11$ & $7.36 \pm 0.34$ & 6 \\
\hline DSLET & 6.72 & $1.33 \pm 0.12$ & $6.96 \pm 0.46$ & 6 \\
\hline U50,488 & 6.83 & $1.34 \pm .037$ & $7.11 \pm 0.47$ & 6 \\
\hline
\end{tabular}

\section{DISCRIMINATIVE STIMULUS EFFECTS IN RHESUS MONKEYS}

NIH 10683 was studied in rhesus monkeys for its discriminative stimulus effects, analgesic effects, and effects on respiratory function. NIH 10683 substituted completely for the opioid $\boldsymbol{\kappa}$ agonist ethylketocyclazocine in one of two monkeys discriminating between saline and $0.032 \mathrm{mg} / \mathrm{kg}$ of ethylketocyclazocine. NM 10683 did not substitute for the opioid $\mu$ agonist alfentanil and also failed to substitute for the opioid antagonist naltrexone in morphine-treated (3.2 $\mathrm{mg} / \mathrm{kg} /$ day) monkeys discriminating between $0.01 \mathrm{mg} / \mathrm{kg}$ of naltrexone and saline. When saline is substituted for the daily injection of morphine in monkeys discriminating between naltrexone and saline, subjects respond on the naltrexone lever; this naltrexone-lever responding is reversed by morphine-like opioids (e.g., 
alfentanil) and appears to be related to opioid withdrawal. Up to a dose of 1.78 $\mathrm{mg} / \mathrm{kg}$, NIH 10683 failed to affect naltrexone-lever responding in morphineabstinent monkeys. Thus, with regard to discriminative stimulus effects, NIH may have $\kappa$ agonist effects and no $\mu$ agonist nor $\mu$ antagonist effects.

\section{ANALGESIC EFFECTS IN RHESUS MONKEYS}

$\mathrm{NIH} 10683$ also was studied for its effects on the latency of monkeys to remove their tails from warm water. Up to the largest dose that could be administered, $10.0 \mathrm{mg} / \mathrm{kg}$, NIH 10683 produced $100 \%$ and $79 \%$ of the maximum possible effect (i.e., 20-sec latency) with 50 and $55^{\circ} \mathrm{C}$ water, respectively. This analgesic effect of NIH 10683 was markedly antagonized by a pretreatment of $1.0 \mathrm{mg} / \mathrm{kg}$ of quadazocine. Whereas under control conditions a dose of $3.2 \mathrm{mg} / \mathrm{kg}$ of NIH 10683 produced a maximum effect with $50^{\circ} \mathrm{C}$ water, in the presence of 1.0 $\mathrm{mg} / \mathrm{kg}$ of quadazocine this dose of NIH 10683 had no effect on tail withdrawal latencies. A smaller dose of quadazocine, $0.1 \mathrm{mg} / \mathrm{kg}$, produced a smaller antagonism of $\mathrm{NIH} 10683$ with $50^{\circ} \mathrm{C}$ water and no antagonism with $55^{\circ} \mathrm{C}$ water.

\section{RESPIRATORY FUNCTION STUDIES IN RHESUS MONKEYS}

The effects of NIH 10683 on respiratory function were studied in a monkey breathing air or $5 \% \mathrm{CO}_{2}$ in air, the effects of $\mathrm{NIH} 10683$ on respiratory function in air when NIH 10683 was administered alone and when administered after pretreatment with $1.0 \mathrm{mg} / \mathrm{kg}$ of the opioid antagonist quadazocine. Up to a dose of $3.2 \mathrm{mg} / \mathrm{kg}$, NIH 10683 produced dose-related decreases in ventilatory frequency (f) and in ventilatory volume $\left(\mathrm{V}_{\mathrm{T}}\right)$ with maximum decreases to $50 \%$ of control at the largest dose studied. Quadazocine attenuated decreases in $\mathrm{f}$ but

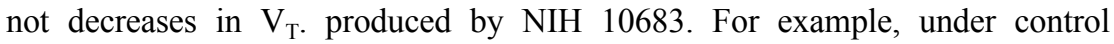
conditions NIH 10683 decreased fin air to $52 \%$ of control; in the presence of quadazocine this dose of NIH 10683 decreased $\mathrm{f}$ to only $86 \%$ of control. Qualitatively similar results were obtained when this subject breathed $5 \% \mathrm{CO}_{2}$ in air.

\section{SELF-ADMINISTRATION STUDIES IN RHESUS MONKEYS}

Doses of from 0.0001 to $0.1 \mathrm{mg} / \mathrm{kg} / \mathrm{inj}$ NIH 10683 were evaluated. NIH 10683 at $0.03 \mathrm{mg} / \mathrm{kg} / \mathrm{inj}$ maintained rates of responding that were slightly below those maintained by the maximum rate-maintaining dose of alfentanil in the two monkeys. No other dose of NIH 10683 maintained response rates above those maintained by saline. 


\section{SUMMARY}

NIH 10683 had a very unusual spectrum of action across the preparations described herein. Its binding results would predict that opioid potency would be roughly comparable to morphine. This prediction was borne out by the in vivo studies. The vas deferens preparation demonstrated that the compound had no opioid agonist characteristics, but NIH 10683 did have antagonist actions against each agonist used in the preparation. These data, taken at face value, might argue for a naloxone-like action with less potency. This supposition was not borne out behaviorally in the naltrexone discrimination studies in monkeys, The other behavioral data suggest opioid agonist activity in some preparations. The compound appeared to have $\kappa$ agonist effects in the drug discrimination assay. However, it suppressed respiratory function by an opioid mechanism. This is inconsistent with $\mathrm{K}$ agonist activity. NIH 10683 was self-injected in the dose range expected from its behavioral protency in other assays.

14-Hydroxy-N-(2-methoxyethyl)-7,8-dihydronorisomorphine

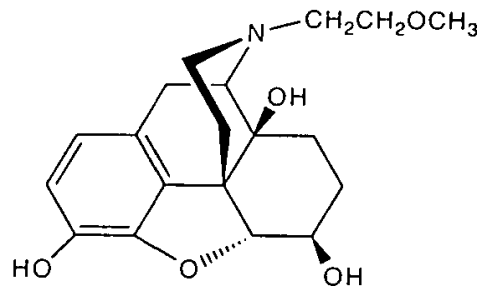

\section{DISPLACEMENT OF $\left[{ }^{3} \mathrm{H}\right]$ ETORPHINE BINDING}

$\mathrm{EC}_{50}$ of $5.83 \mathrm{nM}$ in the presence of 150 $\mathrm{mM} \mathrm{NaCI}$.

MOUSE VAS DEFERENS PREPARATION

\begin{tabular}{||l|l|l|l|l||}
\hline \hline Condition & $\mathrm{EC}_{50}(\mathrm{nM})$ & $\begin{array}{l}\text { Maximum } \\
\text { Response (\%) }\end{array}$ & Shift & $\mathrm{n}$ \\
\hline \hline Control & $98.7 \pm 1.3$ & & & 3 \\
\hline Naltrexone $(100 \mathrm{nM})$ & $29125.0 \pm 3785.0$ & $96.8 \pm 3.2$ & 0.6 & 3 \\
\hline
\end{tabular}


NIH 10684 (continued)

\begin{tabular}{||l|l|l|l|l||}
\hline \hline Agonist & $\mathrm{pA}_{2}$ & Slope \pm S.D. & $\begin{array}{l}\mathrm{pA}_{2}(\text { Constrained }) \pm \\
\mathrm{SE}\end{array}$ & $\mathrm{n}$ \\
\hline \hline Sufentanil & 9.31 & $0.94 \pm 0.21$ & $9.28 \pm 1.27$ & 6 \\
\hline DSLET & 6.92 & $1.14 \pm 0.17$ & $6.98 \pm 0.19$ & 6 \\
\hline U50,488 & 7.49 & $1.22 \pm 0.22$ & $7.60 \pm 0.41$ & 6 \\
\hline \hline
\end{tabular}

\section{DRUG DISCRIMINATION STUDIES IN RHESUS MONKEYS}

In monkeys discriminating between saline and either $0.032 \mathrm{mg} / \mathrm{kg}$ of the opioid $\kappa$ agonist ethylketocyclazocine or $0.056 \mathrm{mg} / \mathrm{kg}$ of the opioid $\mu$ agonist alfentanil, $\mathrm{NIH} 10684$, up to a dose of $10.0 \mathrm{mg} / \mathrm{kg}$, produced only saline-lever responding. In morphine-treated $(3.2 \mathrm{mg} / \mathrm{kg} /$ day $)$ monkeys discriminating between 0.01 $\mathrm{mg} / \mathrm{kg}$ of naltrexone and saline, NIH substituted completely for naltrexone at a dose of $3.2 \mathrm{mg} / \mathrm{kg}$. When saline is substituted for the daily injection of morphine in monkeys discriminating between naltrexone and saline, subjects respond on the naltrexone lever; this naltrexone-lever responding is reversed by morphine-like opioids (e.g., alfentanil) and appears to be related to opioid withdrawal. Up to a dose of $10.0 \mathrm{mg} / \mathrm{kg}$, NIH 10684 had no effect on naltrexone-lever responding in saline-treated (morphine-abstinent) monkeys. However, in morphine-abstinent monkeys NIH 10684 antagonized the withdrawal-reversing effects of alfentanil as evidenced by dose-related shifts to the right in the alfentanil dose-effect curve. Antagonism of alfentanil by NIH 10684 was reversible and appeared to be competitive (slope $=-1.07$ ) and the apparent affinity (i.e., $\mathrm{pA}_{2}$ ) of NIH 10684 for $\mu$ opioid receptors was 6.49 . Thus, with regard to discriminative stimulus effects, NLH 10684 had no apparent opioid agonist effects, but did substitute for the opioid antagonist naltrexone in morphine-treated monkeys. NIH 10684 was 100 times less potent than naltrexone $\left(\mathrm{pA}_{2}=8.69\right)$ in drug discrimination studies. $\mathrm{NIH} 10684$ had no marked effect on rates of lever pressing.

\section{ANALGESIA STUDIES IN RHESUS MONKEYS}

NIH 10684 was also studied for its effects on the latency of monkeys to remove their tails from warm water. Up to a dose of $17.8 \mathrm{mg} / \mathrm{kg} \mathrm{NIH} 10684$ had no effect on tail withdrawal latency; however, a dose of $32.0 \mathrm{mg} / \mathrm{kg}$ produced $44 \%$ and $8 \%$ of the maximum obtainable response $(20 \mathrm{sec})$ with 50 and $55^{\circ} \mathrm{C}$. water, respectively. When administered as a pretreatment, NIH 10684 antagonized the analgesic effects of alfentanil with a dose of $1.0 \mathrm{mg} / \mathrm{kg}$ of NIH 10684 producing a 3-10 fold shift to the right in the alfentanil dose-effect curve. 
NIH 10684 (continued)

\section{STUDIES ON RESPIRATORY FUNCTION IN RHESUS MONKEYS}

The effects of NIH 10684 on respiratory function were studied in a monkey breathing air or $5 \% \mathrm{CO}$, in air. Up to a dose of $32.0 \mathrm{mg} / \mathrm{kg}$, NIH 10684 did not have any consistent effect on ventilatory frequency (f) or ventilatory volume (VT).

\section{SELF-ADMINISTRATION IN RHESUS MONKEYS}

The reinforcing effects of NIH 10684 were evaluated in three monkeys experienced in responding and receiving intravenous infusions of alfentanil. Doses of from 0.003 to $0.3 \mathrm{mg} / \mathrm{kg} /$ inj NIH 10684 were evaluated. NIH 10684 at $0.1 \mathrm{mg} / \mathrm{kg} /$ inj maintained rates of responding slightly below the maximum ratemaintaining dose of alfentanil in monkey RC 213. RC 213 also responded slightly above those rates maintained by 0.0003 alfentanil at $0.03 \mathrm{mg} / \mathrm{kg} / \mathrm{inj} \mathrm{NIH}$ 10684. No other dose maintained rates above the criterion rate for saline in monkey RC 213. NIH 10684 at $0.1 \mathrm{mg} / \mathrm{kg} /$ inj was the only dose at which monkey $\mathrm{CH} 852$ maintained rates above the criterion rate for saline in this experiment. NIH 10684 had virtually no capacity to maintain response rates above those maintained by saline in monkey L 998. Rates for all monkeys peaked at $0.1 \mathrm{mg} / \mathrm{kg} /$ inj $\mathrm{NIH} 10684$.

In summary, NM 10684 was less potent and less efficacious than alfentanil in this procedure. It reliabilty maintained self-injection responding in two of the three monkeys.

\section{SUMMARY}

NIH 10684 was slightly less potent than naltrexone in the binding assay, and more potent than naltrexone, but u-selective, in the vas deferens preparation, It also had complex activity at concentrations above $100 \mathrm{nM}$ which was not blocked by naltrexone. In vivo, NIH 10684 substituted for naltrexone, but was much less potent. This finding suggests that NIH 10684 may not have as effective bioavailability as naltrexone. It was not effective in the thermal analgesia assay. NIH 10684 was an opioid antagonist selective for u-receptor mediated actions in a number of preparations. It did have weakly reinforcing effects, but no other effects that suggested a morphine-like abuse liability. 


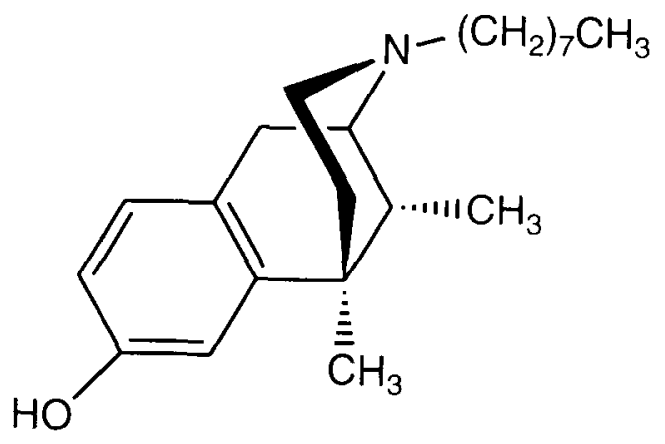

Other in vivo data were presented in the 1992 Annual report

\section{SELECTIVE RECEPTOR BINDING IN MONKEY BRAIN CORTEX}

\begin{tabular}{||c|c|c||}
\hline \hline Assay & $\mathrm{IC}_{50}$ & $\mathrm{r}^{2}$ \\
\hline \hline$\left[{ }^{3} \mathrm{H}\right]$ DAGO $(1.0 \mathrm{nM})$ & 150 & 0.99 \\
\hline$\left[{ }^{3} \mathrm{H}\right] \operatorname{DPDPE}(1.5 \mathrm{nM})$ & 197 & 0.99 \\
\hline$\left[{ }^{3} \mathrm{H}\right] \mathrm{U} 69,593(1.5 \mathrm{nM})$ & 226 & 0.99 \\
\hline
\end{tabular}

NIH 10698

(+)-5,9 $\alpha$-Dimethyl-2'-hydroxy-2-n-octyl-6,7benzomorphan hydro-chloride

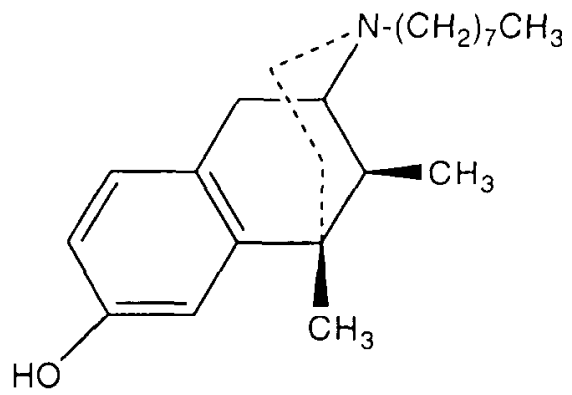

Other in vitro data were presented in the 1992 Annual Report 
NIH 10698 (continued)

SELECTIVE RECEPTOR BINDING IN MONKEY BRAIN CORTEX

\begin{tabular}{||c|c|c|}
\hline \hline Assay & IC $_{50}$ & $\mathrm{r}^{2}$ \\
\hline \hline$\left[{ }^{3} \mathrm{H}\right]$ DAG0 $(1.0 \mathrm{nM})$ & 763 & 0.99 \\
\hline$\left[{ }^{3} \mathrm{H}\right]$ DPDPE $(1.5 \mathrm{nM})$ & $21 \%$ inhibition at $6000 \mathrm{nM}$ \\
\hline$\left[{ }^{3} \mathrm{H}\right] \mathrm{U} 69,593(1.5 \mathrm{nM})$ & 2839 & 0.98 \\
\hline
\end{tabular}

NIH 10700

1 - [ 1 - (2-Hydroxy phenyl)cyclohexyl] -3,4 dehydropiperidine hydro-chloride<smiles>Oc1cccc(C2(N3CC=CCC3)CCCCC2)c1</smiles>

Other in vitro data were presented in the 1992 Annual Report

SELECTIVE RECEPTOR BINDING IN MONKEY BRAIN CORTEX

\begin{tabular}{||c|c|c||}
\hline \hline Assay & $\mathrm{IC}_{50}$ & $\mathrm{r}^{2}$ \\
\hline \hline$\left[{ }^{3} \mathrm{H}\right]$ DAG0 $(1.0 \mathrm{nM})$ & 61.9 & 0.99 \\
\hline$\left[{ }^{3} \mathrm{H}\right]$ DPDPE $(1.5 \mathrm{nM})$ & 2370 & 0.99 \\
\hline$\left[{ }^{3} \mathrm{H}\right] \mathrm{U} 69,593(1.5 \mathrm{nM})$ & 2695 & 0.99 \\
\hline
\end{tabular}




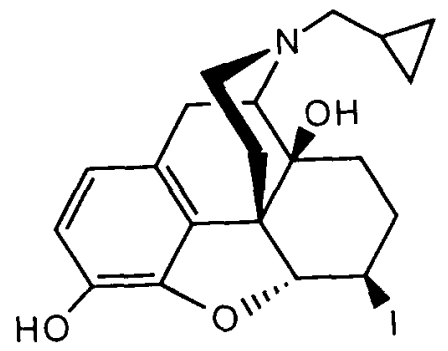

Other in vivo data were presented in the 1992 Annual Report.

SELECTIVE RECEPTOR BINDING IN MONKEY BRAIN CORTEX

\begin{tabular}{||c|c|c||}
\hline \hline Assay & $\mathrm{IC}_{50}$ & $\mathrm{r}^{2}$ \\
\hline \hline$\left[{ }^{3} \mathrm{H}\right]$ DAG0 $(1.0 \mathrm{nM})$ & 1.06 & 0.99 \\
\hline$\left[{ }^{3} \mathrm{H}\right]$ DPDPE $(1.5 \mathrm{nM})$ & 26.0 & 0.99 \\
\hline$\left[{ }^{3} \mathrm{H}\right] \mathrm{U} 69,593(1.5 \mathrm{nM})$ & 0.34 & 0.9 \\
\hline
\end{tabular}

NIH 10702 6 $\alpha$-Iodo-3,14-dihydroxy-17-cyclopropylmethyl$4,5 \alpha$-epoxymorphinan oxalate

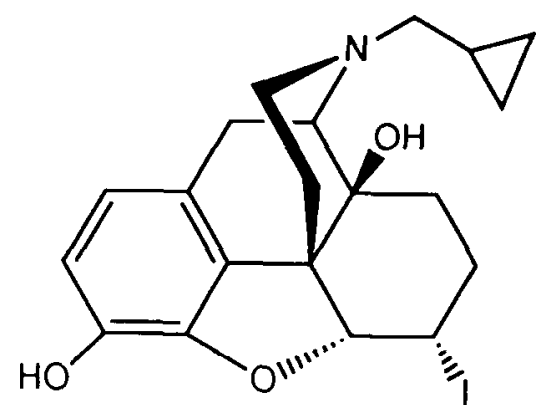

Other in vitro data were presented in the 1992 Annual Report 
NIH 10702 (continued)

\begin{tabular}{||c|c|c||}
\hline Assay & $\mathrm{IC}_{50}$ & $\mathrm{r}^{2}$ \\
\hline \hline$\left[{ }^{3} \mathrm{H}\right]$ DAG0 $(1.0 \mathrm{nM})$ & 0.42 & 0.98 \\
\hline$\left.\Gamma^{3} \mathrm{H}\right]$ DPDPE $(1.5 \mathrm{nM})$ & 9.57 & 0.99 \\
\hline$\left.{ }^{3} \mathrm{H}\right] \mathrm{U} 69,593(1.5 \mathrm{nM})$ & 0.30 & 0.98 \\
\hline
\end{tabular}

* $* *$

NIH $10703 \quad \mathrm{~N}$-[(3,4-Dichlorophenyl)acetyl]-N,2-dimethyl-2(N',N'-dimethylamino) ethylamine oxalate<smiles>CC(CN(C)C(=O)Cc1ccc(Cl)c(Cl)c1)N(C)C</smiles>

Other in vitro data were presented in the 1992 Annual Report.

RECEPTOR SELECTIVE BINDING IN MONKEY BRAIN

\begin{tabular}{||c|c|c||}
\hline \hline Assay & $\mathrm{IC}_{50}$ & $\mathrm{r}^{2}$ \\
\hline \hline$\left[{ }^{3} \mathrm{H}\right]$ DAG0 $(1.0 \mathrm{nM})$ & 1791 & 0.99 \\
\hline$\left[{ }^{3} \mathrm{H}\right]$ DPDPE $(1.5 \mathrm{nM})$ & $6 \%$ inhibition at $6000 \mathrm{nM}$ \\
\hline$\left.{ }^{3} \mathrm{H}\right] \mathrm{U} 69,593(1.5 \mathrm{nM})$ & 287 & 0.99 \\
\hline
\end{tabular}




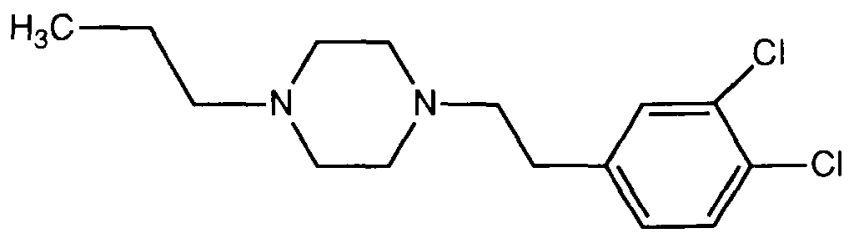

Other in vitro data were presented in the 1992 Annual Report

\section{RECEPTOR SELECTIVE BINDING IN MONKEY BRAIN CORTEX}

\begin{tabular}{||c|c|c||}
\hline Assay & IC $_{50}$ & $\mathrm{r}^{2}$ \\
\hline \hline$\left[{ }^{3} \mathrm{H}\right]$ DAGO $(1.0 \mathrm{nM})$ & 5210 & 0.99 \\
\hline$\left[{ }^{3} \mathrm{H}\right] \mathrm{DPDPE}(1.5 \mathrm{nM})$ & $4 \%$ inhibition at $6000 \mathrm{nM}$ \\
\hline$\left[{ }^{3} \mathrm{H}\right] \mathrm{U} 69,593(1.5 \mathrm{nM})$ & 3181 & 0.99 \\
\hline
\end{tabular}

$* * *$

NIH 10738

4-(3-Hydroxyphenyl)-1-(4-nitrobenzyl)-4-(1oxopropyl) piperidine hydrochloride

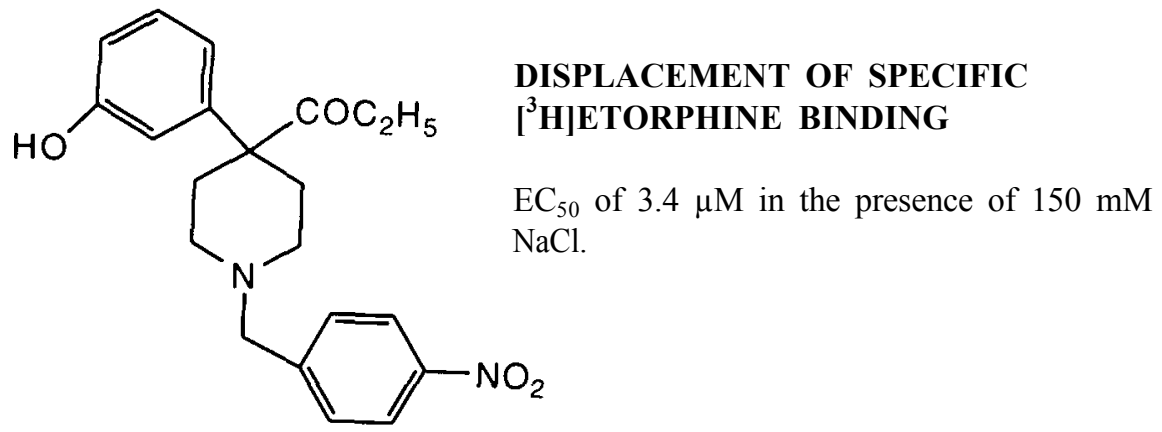


NIH 10738 (continued)

MOUSE VAS DEFERENS PREPARATION

\begin{tabular}{||l|l|l|l|l||}
\hline \hline Agonist & $\mathrm{p} \mathrm{A}_{2}$ & Slope \pm S.D. & $\begin{array}{c}\mathrm{pA}_{2} \\
\left(\begin{array}{c}\text { Constrained }) \\
\pm \mathrm{S} \text { E }\end{array}\right.\end{array}$ & $\mathrm{n}$ \\
\hline \hline Sufentanil $(\mu)$ & $<5.5$ & & & 3 \\
\hline DSLET $(\delta)$ & 6.18 & $1.30 \pm 0.04$ & $6.38 \pm 0.44$ & 6 \\
\hline U50,488 $(\mathbf{k})$ & 5.96 & $1.73 \pm 0.42$ & $6.29 \pm 0.64$ & 6 \\
\hline \hline
\end{tabular}

\section{SUMMARY}

$\mathrm{NIH} 10738$ was of low potency in both preparations In the vas deferens preparation it was devoid of opioid agonist activity. Concentrations of 10 and $30 \mu \mathrm{M}$ markedly increased the magnitude of the twitch, an action that was not blocked by $100 \mathrm{nM}$ of naltrexone. NIH 10738 was an antagonist of low potency on this preparation. It was equipotent as an antagonist at $\delta$ and $\boldsymbol{\kappa}$ opioid receptors. At a concentration of $10 \mu \mathrm{M}$, the compound caused a 9.02fold shift in the concentration-effect curve. Because of its low potency and direct actions on the smooth muscle preparation, $\mathrm{pA}_{2}$ values could not be calculated for its antagonist activity at $\mu$ opioid receptors. The high slopes of the Schild plots suggest that the antagonism produced by NIH 10738 is not a simple competitive-type of antagonism.

NIH 10739

1-(4-Fluorobenzyl-4-(3-hydroxybenzyl)-4-

(1-oxopropyl) piperidine hydrochloride<smiles>CCOC(=O)C1(c2cccc(O)c2)CCN(Cc2ccc(F)cc2)CC1</smiles>

DISPLACEMENT OF SPECIFIC $\left[{ }^{3}\right.$ H]ETORPHINE BINDING

$\mathrm{EC}_{50}$ of $1330 \mathrm{nM}$ in the presence of $150 \mathrm{mM}$

$\mathrm{NaC} 1$. 
NIH 10739 (continued)

MOUSE VAS DEFERENS PREPARATION

\begin{tabular}{||l|l|l|l|l||}
\hline \hline Agonist & $\mathrm{pA}_{2}$ & Slope \pm S.D. & $\begin{array}{c}\mathrm{pA}_{2} \\
\left(\begin{array}{c}\text { Constrained }) \\
\pm \text { S.E. }\end{array}\right.\end{array}$ & $\mathrm{n}$ \\
\hline Sufentanil $(\mu)$ & $<5.0$ & & & 4 \\
\hline DSLET $(\boldsymbol{\delta})$ & 6.37 & $1.66 \pm 0.16$ & $6.94 \pm 0.59$ & 6 \\
\hline U50,488 $\boldsymbol{\kappa})$ & 5.71 & $1.82 \pm 0.52$ & $5.88 \pm 0.69$ & 6 \\
\hline \hline
\end{tabular}

\section{SUMMARY}

NIH 10739 was not very potent in either preparation. It was devoid of opioid agonist activity on the isolated, electrically stimulated mouse vas deferens preparation. Concentrations of 10 and $30 \mu \mathrm{M}$ markedly increased the magnitude of the twitch, an action which was not blocked by $100 \mathrm{nM}$ naltrexone, NIH 10739 was an antagonist of low potency on this preparation. It was ten times more potent as an antagonist at $\delta$ opioid receptors than at $\kappa$ opioid receptors. Although NIH 10739 did not shift the sufentanil concentration-effect curve to the right, it significantly decreased the maximum inhibitory action of sufentanil. NIH 10739, at a concentration of $10 \mu \mathrm{M}$, reduced the maximum response to sufentanil to $43.1 \%$ of control values. The high slopes of the Schild plots and the changes in the maximum responses to sufentanil suggest that the antagonist produced by NIH 10739 is not competitive.

( \pm )-cis-N-[3-methyl-1-[2-oxo-2-(2-thienyl)ethyl]-4piperidinyl]-N-phenylpropanamide hydrochloride<smiles>CCC(=O)N(c1ccccc1)[C@H]1CCN(CC(=O)c2cccs2)C[C@H]1C</smiles>

DISPLACEMENT OF SPECIFIC [ $\left.{ }^{3} \mathrm{H}\right]$ ETORPHINE BINDING

$\mathrm{EC}_{50}$ of $569 \mathrm{nM}$ in the presence of $150 \mathrm{mM} \mathrm{NaCl}$. 
NIH 10762 (continued)

\section{MOUSE VAS DEFERENS PREPARATION}

Concentrations up to $3 \mu \mathrm{M}$ had no appreciable effect on this preparation. At a concentration of $30 \mu \mathrm{M}$, NIH 10762 markedly increased the magnitude of the twitch, an effect that was not modified by $100 \mathrm{nM}$ naltrexone. When tested as an antagonist, $30 \mu \mathrm{M}$ NIH 10762 caused a

6.86-fold shift to the right in the sufentanil concentration-effect curve. Because of the low potency of this drug as a $\mu$ opioid receptor antagonist, $\mathrm{pA}_{2}$ values could not be determined. NIH 10762,30 $\mu \mathrm{M}$, did not affect responses of the vas deferens to DSLET or to U50,488. Thus, NIH 10762 appears to be a $\mu$ opioid receptor antagonist of very low potency and to be devoid of opioid agonist activity on the mouse vas deferens preparation.

\section{SUMMARY}

NIH 10762 had low potency in the binding assay and some possible opioid antagonist activity in the mouse vas deferens preparation.

NIH 10765

(土)-cis-N-[1-(2-Hydroxy-1-phenylethyl)-3-methyl4-piperidinyl]-N-phenylpropanamide hydrochloride

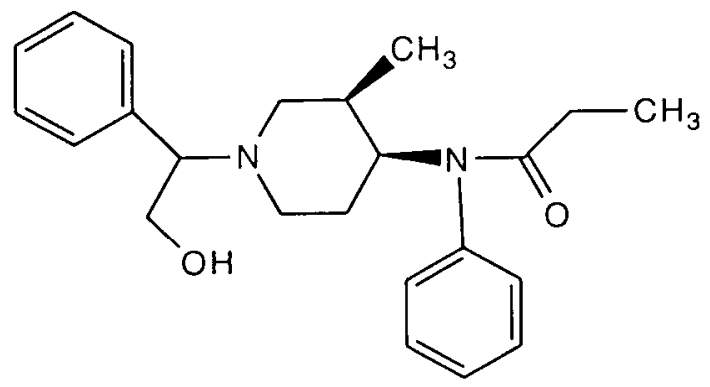

DISPLACEMENT OF $\left[{ }^{3}\right.$ H]ETORPHINE BINDING

$\mathrm{EC}_{50}$ of $3.4 \mu \mathrm{M}$ in the presence of $150 \mathrm{mM} \mathrm{NaCl}$.

\section{MOUSE VAS DEFERENS PREPARATION}

NIH 10765 was studied on the isolated, electrically stimulated mouse vas deferens preparation in concentrations which ranged from $1 \mathrm{nM}$ to $30 \mu \mathrm{M}$. Concentrations up to $3 \mu \mathrm{M}$ had no appreciable effect on this preparation. At a concentrations of $30 \mu \mathrm{M}$ NIH 10765 markedly increased the magnitude of the twitch, an effect that was not modified by $100 \mathrm{nM}$ naltrexone. When tested as an 
antagonist $30 \mu \mathrm{M}$ NIH 10765 caused a 9.53-fold shift to the right in the sufentanil concentration-effect curve. Because of the low potency of this drug as a $\mu$-opioid receptor antagonist, $\mathrm{pA}_{2}$ vaues could not be determined. NIH $10765,30 \mu \mathrm{M}$, did not affect responses of the vas deferens to DSLET, a $\delta$ opioid receptor agonist, or to U50,488, a $\kappa$ receptor selective antagonist. Thus, in the vas deferens preparation, NIH 10765 appears to be a $\mu$-opioid receptor antagonist of very low potency and to be devoid of agonist-activity.

\section{SUMMARY}

NIH 10765 had insignificant opioid activity in both in vivo assays

NIH 10773

Morphine 3-acetate 6-sulfate zwitterion

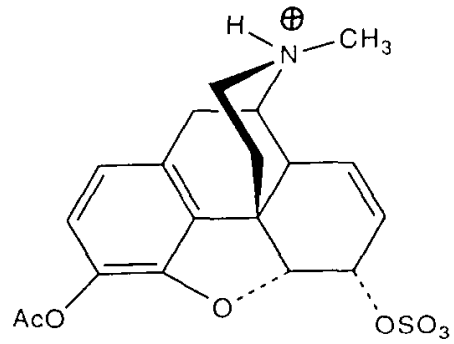

DISPLACEMENT OF SPECIFIC $\left[{ }^{3}\right.$ H]ETORPHINE BINDING

$\mathrm{EC}_{50}$ of $517 \mathrm{nM}$ in the presence of $150 \mathrm{mM}$ $\mathrm{NaCl}$.

MOUSE VAS DEFERENS PREPARATION

\begin{tabular}{||c|c|c|c|c||}
\hline Condition & $\mathrm{EC}_{50}(\mathrm{nM})$ & $\begin{array}{l}\text { Maximum } \\
\text { Response }(\% 0\end{array}$ & $\begin{array}{l}\text { Shift } \\
\text { (x-fold) }\end{array}$ & $\mathrm{n}$ \\
\hline \hline $\begin{array}{c}\text { Control } \\
\text { Naltrexone } \\
(100 \mathrm{nM})\end{array}$ & $836.5 \pm 119.7$ & $94.8 \pm 2.8$ & 6.0 & 3 \\
\hline $\begin{array}{c}\text { ICI-174,864 } \\
(100 \mathrm{nM})\end{array}$ & $127.6 \pm 25.1$ & $87.3 \pm 2.7$ & 0.9 & 3 \\
\hline $\begin{array}{l}\text { Nor-BNI } \\
(10 \mathrm{nM})\end{array}$ & $194.9 \pm 12.9$ & $65.0 \pm 2.4$ & 1.4 & 3 \\
\hline
\end{tabular}


NIH 10773 (continued)

\section{SUMMARY}

$\mathrm{NIH} 10773$ was weakly active in both preparations. In the mouse vas deferens preparation, it acted as a partial inhibitor of the twitch, with actions mediated predominantly by $\mu$ opioid receptors. No evidence of opioid antagonist activity was found.

NIH 10782

\section{3-[4-Methylpiperazinylliminomethyl]} rifamycin (Rifampin, or Rifampicin)

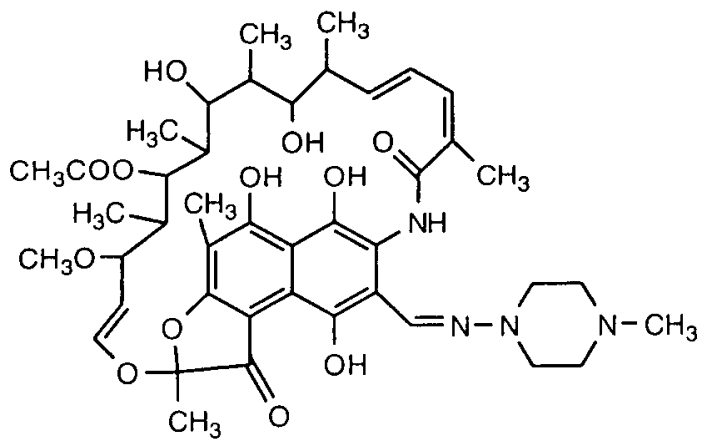

\section{DISPLACEMENT OF SPECIFIC $\left[{ }^{3}\right.$ H]ETORPHINE BINDING}

$\mathrm{EC}_{50}$ of $>6000 \mathrm{nM}(2 \%$ inhibition at $6 \mu \mathrm{M})$ in the presence of $\mathrm{NaCl}$

MOUSE VAS DEFERENS PREPARATION

\begin{tabular}{||c|c|c|c|c||}
\hline Condition* & $\mathrm{EC}_{50}(\mathrm{nM})$ & $\begin{array}{c}\text { Maximum } \\
\text { Response (\%) }\end{array}$ & $\begin{array}{l}\text { Shift } \\
(\mathrm{x} \text {-fold) }\end{array}$ & $\mathrm{n}$ \\
\hline Control & $36.9 \pm 13.2$ & $29.6 \pm 5.8$ & 3.7 & 3 \\
\hline $\begin{array}{c}\text { Naltrexone } \\
(100 \mathrm{nM})\end{array}$ & $309.5 \pm 90.8$ & $20.1 \pm 2.9$ & 5.7 & 3 \\
\hline
\end{tabular}

* NOTE: Evaluated as a $3 \mathrm{mM}$ solution in $32 \%$ ethanol.

\section{SUMMARY}

$\mathrm{NIH} 10782$ had no significant activity in the binding assay. In the mouse vas deferens preparation it acted as an agonist of low potency that might act on $\mu$ 
NIH 10782 (continued)

opioid receptors. When tested in a concentration of $10 \mu \mathrm{M}$ as an antagonist, it failed to block the actions of sufentanil, DSLET or U50,488.

NIH 10785

(土)-cis-N-[1-(2-Hydroxy-2-phenylethyl)-3-methyl4-piperidinyll-N-(4-fluorophenyl)propanamide hydrochloride<smiles>CCC(=O)N(c1ccc(F)cc1)[C@@H]1CCN(C[C@H](O)c2ccccc2)C[C@H]1C</smiles>

DISPLACEMENT OF SPECIFIC [ ${ }^{3}$ H]ETORPHINE BINDING

$\mathrm{EC}_{50}$ of $4.54 \mathrm{nM}$ in the presence of $150 \mathrm{mM} \mathrm{NaCI}$.

MOUSE VAS DEFERENS PREPARATION

\begin{tabular}{||c|c|c|c|c||}
\hline \hline Condition & $\mathrm{EC}_{50}(\mathrm{nM})$ & $\begin{array}{c}\text { Maximum } \\
\text { Response (\%) }\end{array}$ & $\begin{array}{l}\text { Shift } \\
\text { (x-fold) }\end{array}$ & $\mathrm{n}$ \\
\hline \hline Control & $2.83 \pm 0.61$ & 100 & 21.2 & 3 \\
\hline $\begin{array}{l}\text { Naltrexone } \\
(100 \mathrm{nM})\end{array}$ & $60.0 \pm 18.8$ & 100 & 17.6 & 3 \\
\hline $\begin{array}{l}\text { ICI } 174,864 \\
(100 \mathrm{nM})\end{array}$ & $50.0 \pm 9.30$ & 100 & 2.4 & 3 \\
\hline $\begin{array}{l}\text { Nor-BNI } \\
(10 \mathrm{nM})\end{array}$ & $6.87 \pm 1.24$ & 100 & & 9 \\
\hline
\end{tabular}

\section{SUMMARY}

NIH 10785 had significant opioid activity in the binding assay and, in the mouse vas deferens preparation, it acted as a potent agonist with both naltrexone and ICI 174,864 causing shifts to the right in the NIH 10785 concentration-effect curve. Nor-binaltorphimine neither shifted the NIH 10785 concentration-effect curve appreciably nor significantly altered the maximum response. Thus, NIH 10785 would seem to be an agonist with actions at $\delta$ opioid receptors. 
<smiles>CCC(=O)N(c1ccccc1)[C@H]1CCN(C[C@H](O)c2ccc(Br)cc2)C[C@H]1C</smiles>

DISPLACEMENT OF SPECIFIC [ ${ }^{3}$ H]ETORPHINE BINDING

$\mathrm{EC}_{50}$ of $22.9 \mathrm{nM}$ in the presence of $150 \mathrm{mM} \mathrm{NaCl}$

\section{MOUSE VAS DEFERENS PREPARATION}

\begin{tabular}{||c|c|c|c|c||}
\hline Condition & $\mathrm{EC}_{50}(\mu \mathrm{M})$ & $\begin{array}{l}\text { Maximum } \\
\text { Response }(\%)\end{array}$ & $\begin{array}{l}\text { Shift } \\
(\mathrm{x} \text {-fold })\end{array}$ & $\mathrm{n}$ \\
\hline Control & $1.44 \pm 0.35$ & $98.8 \pm 0.6$ & 2.4 & 3 \\
\hline $\begin{array}{l}\text { Naltrexone } \\
(100 \mathrm{nM})\end{array}$ & $3.47 \pm 0.88$ & 100 & 7.2 & 3 \\
\hline $\begin{array}{l}\text { ICI } 174,864 \\
(100 \mathrm{nM})\end{array}$ & $10.3 \pm 9.3$ & $99.1 \pm 0.9$ & 0.0 & 3 \\
\hline $\begin{array}{l}\text { Nor-BNI } \\
(10 \mathrm{nM})\end{array}$ & $0.07 \pm 0.03$ & $31.8 \pm 2.4$ & & 3 \\
\hline
\end{tabular}

\begin{tabular}{||c|c|c|c|c||}
\hline Agonist & $\mathrm{pA}_{2}$ & Slope \pm S.D. & $\begin{array}{c}\mathrm{pA}_{2} \text { (Constrained) } \\
\pm \text { SE }\end{array}$ & $\mathrm{n}$ \\
\hline Sufentanil $(\mu)$ & 5.98 & $1.13 \pm 0.10$ & $6.04 \pm 0.38$ & 3 \\
\hline
\end{tabular}

\section{SUMMARY}

NIH 10786 had significant opioid activity in the binding assay. In the mouse vas deferens preparation, it had complex agonist actions. Because the concentrationeffect curve appears to be multi-phasic, interpretation of results is difficult. Both naltrexone and ICI 174,864 caused shifts to the right in the NIH 10786 concentration effect curve. Nor-binaltorphimine markedly decreased the maximum response obtained with NIH 10786. Concentrations of NIH 10786 above $1 \mu \mathrm{M}$ markedly increased the magnitude of the twitch in the presence of nor-binaltorphimine. NIH 10786 also acted as a weak antagonist. At a 
NIH 10786 (continued)

concentration of $3 \mu \mathrm{M}$, NIH 10786 caused a 4.07-fold shift in the DSLET concentration-effect curve and a 5.3-fold shift in the U50,488 concentration effect curve.

NIH 10787 1-Chloronaltrexone hydrochloride

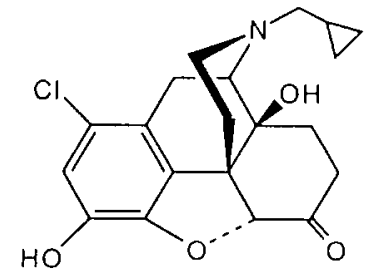

DISPLACEMENT OF SPECIFIC $\left[{ }^{3}\right.$ H]ETORPHINE BINDING

$\mathrm{EC}_{50}$ of $0.38 \mathrm{nM}$ in presence of 150 $\mathrm{mM} \mathrm{NaCI}$.

MOUSE VAS DEFERENS PREPARATION

\begin{tabular}{||c|c|c|c|c||}
\hline Agonist & $\mathrm{pA}_{2}$ & Slope \pm S.D. & $\begin{array}{l}\mathrm{pA}_{2}(\text { Constrained }) \\
\pm \text { S.E. }\end{array}$ & $\mathrm{n}$ \\
\hline \hline Sufentanil $(\mu)$ & 9.49 & 0.96 & $9.46 \pm 0.32$ & 3 \\
\hline DSLET $(\delta)$ & 8.73 & 1.05 & $8.77 \pm 0.36$ & 3 \\
\hline $\mathrm{U} 50,488(\mathbf{k})$ & 894 & 1.08 & $8.98 \pm 0.38$ & 3 \\
\hline \hline
\end{tabular}

\section{SUMMARY}

NIH 10787 had high potency in the binding assay. In the vas deferens preparation it had potent opioid antagonist activity with slight selectivity for $\mu$ opioid receptors. 

oxopropyl)phenyl amino]-r-4-piperidinecarboxylic acid methyl ester hydrochloride

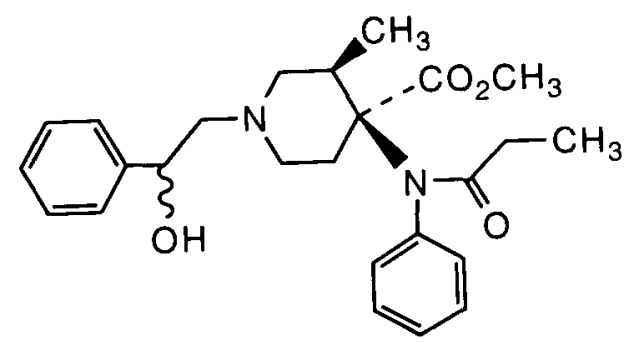

\section{DISPLACEMENT OF SPECIFIC $\left[{ }^{3}\right.$ H]ETORPHINE BINDING}

$\mathrm{EC}_{50}$ of $0.85 \mathrm{nM}$ in the presence of $150 \mathrm{mM} \mathrm{NaCl}$.

MOUSE VAS DEFERENS PREPARATION

\begin{tabular}{||c|c|c|c|c||}
\hline \hline Condition & $\mathrm{EC}_{50}(\mathrm{nM})$ & $\begin{array}{l}\text { Maximum } \\
\text { Response (\%) }\end{array}$ & $\begin{array}{l}\text { Shift } \\
\text { (x-fold) }\end{array}$ & $\mathrm{n}$ \\
\hline \hline Control & $0.77 \pm 0.09$ & 100 & & 9 \\
\hline $\begin{array}{c}\text { Naltrexone } \\
(100 \mathrm{nM})\end{array}$ & $12.4 \pm 2.2$ & 100 & 16.0 & 3 \\
\hline $\begin{array}{l}\mathrm{ICI} 174,864 \\
(100 \mathrm{nM})\end{array}$ & $1.64 \pm 0.76$ & 100 & 2.1 & 3 \\
\hline $\begin{array}{l}\text { Nor-BNI } \\
(10 \mathrm{nM})\end{array}$ & $1.55 \pm 0.58$ & 100 & 2.0 & 3 \\
\hline
\end{tabular}

\section{SUMMARY}

NIH 10790 had high affinity in the binding assay and, in the mouse vas deferens preparation, it acted as a potent agonist with some selectivity for $\mu$ opioid receptors. 


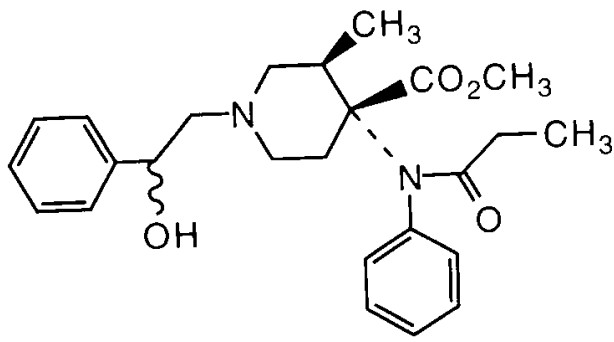

\section{DISPLACEMENT OF SPECIFIC $\left[{ }^{3}\right.$ H]ETORPHINE BINDING}

$\mathrm{EC}_{50}$ of $2.29 \mathrm{nM}$ in presence of $150 \mathrm{mM} \mathrm{NaCl}$.

MOUSE VAS DEFERENS PREPARATION

\begin{tabular}{||c|c|c|c|c||}
\hline Condition & $\mathrm{EC}_{50}(\mathrm{nM})$ & $\begin{array}{l}\text { Maximum } \\
\text { Response (\%) }\end{array}$ & $\begin{array}{l}\text { Shift } \\
\text { (s-fold) }\end{array}$ & $\mathrm{n}$ \\
\hline \hline $\begin{array}{l}\text { Control } \\
\text { Naltrexone } \\
(100 \mathrm{nM})\end{array}$ & $23.3 \pm 3.4$ & 100 & 17.8 & 3 \\
\hline $\begin{array}{l}\mathrm{ICI} 174,864 \\
(100 \mathrm{nM})\end{array}$ & $1.96 \pm 0.80$ & 100 & 1.5 & 3 \\
\hline $\begin{array}{l}\text { Nor-BNI } \\
(10 \mathrm{nM})\end{array}$ & $1.28 \pm 0.22$ & 100 & 1.0 & 3 \\
\hline
\end{tabular}

NIH 10791 had high affinity in the binding assay and, in the mouse vas deferens preparation, it acted as a potent agonist with some selectivity for $\mu$ opioid receptors.

\section{SUMMARY}

NIH 10791 had high affinity in the binding assay and, in the mouse vas deferens preparation, it acted as a potent agonist with some selectivity for $\mu$ opioid receptors. 

oxopropyl)phenyl amino]-r-4-piperidinecarboxylic acid ethyl ester hydrochloride

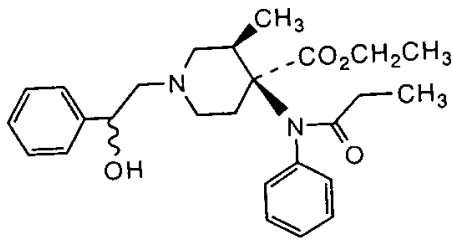

\section{DISPLACEMENT SPECIFIC [ $\left.{ }^{3} \mathrm{H}\right] \mathrm{ETOR}$ - PHINE BINDING}

$\mathrm{EC}_{50}$ of $1.19 \mathrm{nM}$ in the presence of $150 \mathrm{mM} \mathrm{NaCl}$.

MOUSE VAS DEFERENS PREPARATION

\begin{tabular}{||c|c|c|c|c||}
\hline \hline Condition & $\mathrm{EC}_{50}(\mathrm{nM})$ & $\begin{array}{l}\text { Maximum } \\
\text { Response (\%) }\end{array}$ & $\begin{array}{l}\text { Shift } \\
(\mathrm{x} \text {-fold })\end{array}$ & $\mathrm{n}$ \\
\hline \hline Control & $1.29 \pm 0.26$ & 100 & 9.9 & 3 \\
\hline $\begin{array}{l}\text { Naltrexone } \\
(100 \mathrm{nM})\end{array}$ & $12.7 \pm 2.4$ & 100 & 1.6 & 3 \\
\hline $\begin{array}{l}\mathrm{ICI} 174,864 \\
(100 \mathrm{nM})\end{array}$ & $2.08 \pm 0.68$ & 100 & 0.7 & 3 \\
\hline $\begin{array}{c}\text { Nor-BNI } \\
(10 \mathrm{nM})\end{array}$ & $0.93 \pm 0.17$ & 100 & & 9 \\
\hline
\end{tabular}

\section{SUMMARY}

NIH 10792 had high affinity in the binding assay and, in the mouse vas deferens preparation, it acted as a potent agonist with some selectivity for $\mu$ opioid receptors.

NIH $10793 \quad$ ( \pm )-N-[1-(2-Hydroxy-2-phenylethyl)-4methoxymethyl-c-3-methyl-4-4-piperidinyl]-Nphenylpropanamide oxalate<smiles>CCC(=O)N(c1ccccc1)[C@]1(C)CCN(CC(O)c2ccccc2)C[C@@H]1C</smiles>

DISPLACEMENT SPECIFIC $\left[{ }^{3} \mathrm{H}\right] \mathrm{ETOR}$ PHINE BINDING

$\mathrm{EC}_{50}$ of $0.78 \mathrm{nM}$ in the presence of $150 \mathrm{mM} \mathrm{NaCl}$. 
NIH 10793 (continued)

MOUSE VAS DEFERENS PREPARATION

\begin{tabular}{||c|c|c|c|c||}
\hline Condition & $\mathrm{EC}_{50}(\mathrm{nM})$ & $\begin{array}{l}\text { Maximum } \\
\text { Response }(\%)\end{array}$ & $\begin{array}{l}\text { Shift } \\
\text { (x-fold) }\end{array}$ & $\mathrm{n}$ \\
\hline \hline Control & $0.84 \pm 0.15$ & 100 & 23.5 & 3 \\
\hline $\begin{array}{l}\text { Naltrexone } \\
(100 \mathrm{nM})\end{array}$ & $19.7 \pm 6.0$ & 100 & 2.2 & 3 \\
\hline $\begin{array}{l}\text { ICI } 174,864 \\
(100 \mathrm{nM})\end{array}$ & $1.81 \pm 0.63$ & 100 & 4.5 & 3 \\
\hline $\begin{array}{l}\text { Nor-BNI } \\
(10 \mathrm{nM})\end{array}$ & $3.82 \pm 1.04$ & 100 & & 3 \\
\hline \hline
\end{tabular}

\section{SUMMARY}

NIH 10793 had high affinity in the binding assay and, in the mouse vas deferens preparation, it acted as a potent agonist with some selectivity for $\mu$ opioid receptors.

NIH 10802

(土)-3-Hydroxy-N-(4-hydroxybenzyl)morphinan hydrobromide

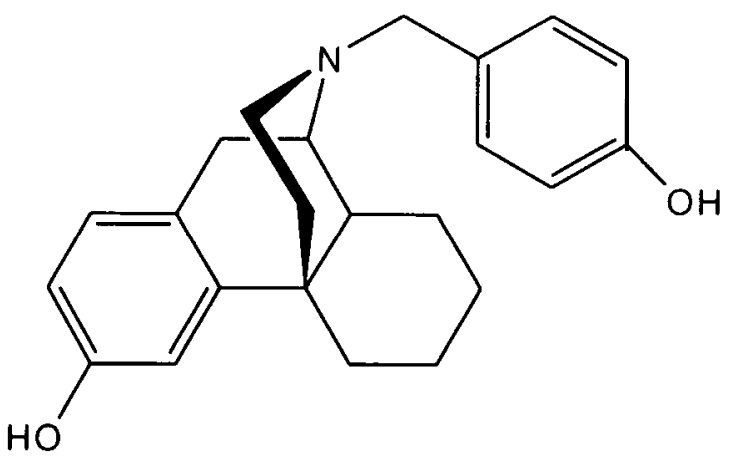

DISPLACEMENT OF SPECIFIC [ $\left.{ }^{3} \mathrm{H}\right] \mathrm{ETORPHINE} \mathrm{BINDING}$

$\mathrm{EC}_{50}$ of $2140 \mathrm{nM}$ in presence of $150 \mathrm{mM} \mathrm{NaCl}$. 
NIH 10802 (continued)

MOUSE VAS DEFERENS PREPARATION

\begin{tabular}{||c|c|c|c|c||}
\hline Condition & $\mathrm{EC}_{50}(\mathrm{nM})$ & $\begin{array}{l}\text { Maximum } \\
\text { Response (\%) }\end{array}$ & $\begin{array}{l}\text { Shift } \\
\text { (x-fold) }\end{array}$ & $\mathrm{n}$ \\
\hline Control & $108.4 \pm 43.6$ & $22.9 \pm 1.7$ & 1.1 & 3 \\
\hline $\begin{array}{l}\text { Naltrexone } \\
(100 \mathrm{nM})\end{array}$ & $113.9 \pm 51.7$ & $22.1 \pm 0.6$ & & 3 \\
\hline
\end{tabular}

\section{SUMMARY}

NIH 10802 had very low affinity in the binding assay. In the mouse vas deferens preparation it was devoid of opioid agonist actions. When tested as an antagonist, NIH 10802 produced unusual results. It blocked responses of the vas deferens to sufentanil ( $\mu$ opioid receptor agonist). The $\mathrm{pA}_{2}$ value for this action was $5.5 \pm 1.4$ and the slope of the Schild plot was $2.78 \pm 1.4$. This high slope indicates that the antagonism is not simply competitive. NIH 10802, $10 \mathrm{nM}$, did not cause significant shifts in the concentration-effect curves for DSLET, a $\delta$ opioid receptor agonist, or to U50,488, a א--opioid receptor agonist.

NIH 10803

( \pm )-N-Allylmecamylamine hydrochloride

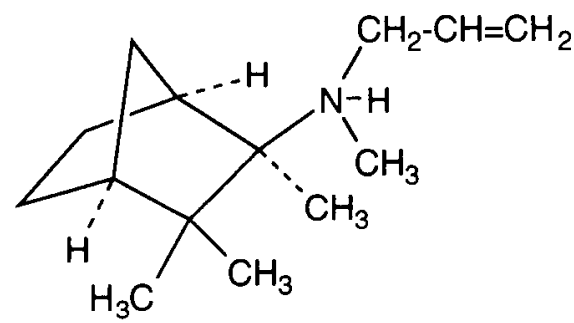

\section{DISPLACEMENT OF SPECIFIC [ ${ }^{3}$ H]ETORPHINE BINDING}

$\mathrm{EC}_{50}$ of $>6,000 \mathrm{nM}(45 \%$ inhibition at $6 \mu \mathrm{M})$ in the presence of $150 \mathrm{mM} \mathrm{NaCl}$. 
NIH 10803 (continued)

MOUSE VAS DEFERENS PREPARATION

\begin{tabular}{||c|c|c|c|c||}
\hline \hline Condition & $\mathrm{EC}_{50}(\mathrm{nM})$ & $\begin{array}{l}\text { Maximum } \\
\text { Response (\%) }\end{array}$ & $\begin{array}{l}\text { Shift } \\
\text { (x-fold) }\end{array}$ & $\mathrm{n}$ \\
\hline \hline Control & $1.83 \pm 0.52$ & $22.9 \pm 1.7$ & & 3 \\
\hline $\begin{array}{c}\text { Naltrexone } \\
(100 \mathrm{nM})\end{array}$ & $0.69 \pm 0.09$ & $12.0 \pm 1.2$ & 0.4 & 3 \\
\hline
\end{tabular}

\section{SUMMARY}

NIH 10803 had no significant activity in the binding assay. In the mouse vas deferens preparation it acted as a quite weak partial agonist or a non-opioid agonist. Naltrexone, $100 \mathrm{nM}$, decreased the maximum response of this drug, but did not shift its concentration-effect curve. When tested as an antagonist, $30 \mu \mathrm{M}$ NIH 10803 did not affect responses of the vas deferens to sufentnail ( $\mu$ opioid agonist), DSLET ( $\delta$ opioid agonist) $\underset{*}{*} \mathrm{U}_{*} 0_{*}^{4} 488$ ( $\kappa$ opioid antagonist).

NIH 10805

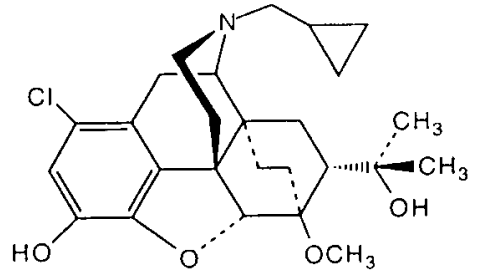

1-Chlorodiprenorphine oxalate

DISPLACEMENT OF SPECIFIC $\left[{ }^{3}\right.$ H]ETORPHINE BINDING

$\mathrm{EC}_{50}$ of $0.61 \mathrm{nM}$ in the presence of $150 \mathrm{mM}$ $\mathrm{NaCl}$.

MOUSE VAS DEFERENS PREPARATION

\begin{tabular}{||c|c|c|c|c||}
\hline Condition & $\mathrm{EC}_{50}(\mathrm{nM})$ & $\begin{array}{l}\text { Maximum } \\
\text { Response (\%) }\end{array}$ & $\begin{array}{l}\text { Shift } \\
\text { (x-fold) }\end{array}$ & $\mathrm{n}$ \\
\hline \hline Control & $1.04 \pm 0.10$ & $272 \pm 0.6$ & & 3 \\
\hline $\begin{array}{l}\text { Naltrexone } \\
(100 \mathrm{nM})\end{array}$ & $134 \pm 0.44$ & $27.9 \pm 1.5$ & 1.3 & 3 \\
\hline \hline
\end{tabular}


NIH 10805 (continued)

\begin{tabular}{||c|c|c|c|c||}
\hline \hline Agonist & $\mathrm{pA}_{2}$ & $\begin{array}{l}\text { Slope } \pm \\
\text { S.D. }\end{array}$ & $\begin{array}{c}\mathrm{pA}_{2} \text { (Constrained) } \\
\text { S.E. }\end{array}$ & $\mathrm{n}$ \\
\hline \hline Sufentanil $(\mu)$ & 9.91 & 1.34 & $10.21 \pm 0.46$ & 3 \\
\hline DSLET $(\delta)$ & 9.27 & 0.85 & $9.01 \pm 1.13$ & 3 \\
\hline U50,488 $(\boldsymbol{\kappa})$ & \multicolumn{3}{|c}{ SEE BELOW } \\
\hline
\end{tabular}

\section{SUMMARY}

NIH 10805 had high potency in the binding assay. In the vas deferens preparation, $\mathrm{MH} 10805$, in concentrations of $0.1 \mathrm{nM}$ to $100 \mathrm{nM}$ decreased the magnitude of the twitch of the electrically stimulated mouse vas deferens preparation. This response was not blocked by naltrexone. Concentrations above $100 \mathrm{nM}$ markedly increased the magnitude of the twitch.

NIH 10805 was a very potent antagonist at $\mu, \delta$, and $\kappa$ receptors. The antagonism of U50,488 was insurmountable and began at a concentration of 1 $\mathrm{nM}$. Although the antagonism of DSLET and sufentanil appeared to be competitive, because of the population of spare $\delta$ and $\mu$ receptors in this preparation, an insurmountable antagonism might have been revealed if very high concentrations of NIH 10805 were studied.

NIH 10809 N-Cyclopropylmethyl[7 $\left.\alpha, 8 \alpha, 2^{\prime}, 3^{\prime}\right]$ cyclopentano-1'$[R]$ hydroxy-6,14-endoethenotetrahydro-

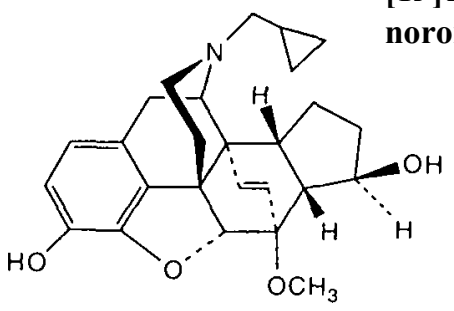

\section{DISPLACEMENT OF SPECIFIC} $\left[{ }^{3}\right.$ H]ETORPHINE BINDING

$\mathrm{EC}_{50}$ of $0.49 \mathrm{nM}$ in the presence of $150 \mathrm{mM}$ $\mathrm{NaCl}$. 
NIH 10809 (continued)

MOUSE VAS DEFERENS PREPARATION

\begin{tabular}{||c|c|c|c|c||}
\hline Condition & $\mathrm{EC}_{50}(\mathrm{nM})$ & $\begin{array}{l}\text { Maximum } \\
\text { Response }(\%)\end{array}$ & $\begin{array}{l}\text { Shift } \\
\text { (x-fold) }\end{array}$ & $\mathrm{n}$ \\
\hline \hline Control & $2.21 \pm 0.17$ & $74.2 \pm 1.8$ & 12.5 & 3 \\
\hline $\begin{array}{l}\text { Naltrexone } \\
(100 \mathrm{nM})\end{array}$ & $27.6 \pm 4.8$ & $80.4 \pm 2.4$ & 1.5 & 3 \\
\hline $\begin{array}{l}\text { ICI } 174,864 \\
(100 \mathrm{nM})\end{array}$ & $3.31 \pm 1.22$ & $75.9 \pm 3.4$ & 18.1 & 3 \\
\hline $\begin{array}{l}\text { Nor-BNI } \\
(10 \mathrm{nM})\end{array}$ & $40.1 \pm 7.42$ & $69.9 \pm 0.7$ & & 3 \\
\hline \hline
\end{tabular}

\section{SUMMARY}

NIH 10809 had high affinity in the binding assay and, in the mouse vas deferens preparation, acted as an agonist relatively selective for $\boldsymbol{\kappa}$ opioid receptors.

NIH $10810 \quad$ N-Cyclopropylmethyl[7 $\alpha, 8 \alpha, 2^{\prime}, 3^{\prime}$ ']cyclopentano-1'$[S]$ hydroxy-6,14-endoethenotetrahydronororipavine hydrochloride

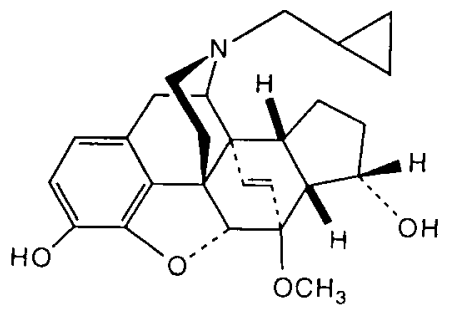

DISPLACEMENT OF SPECIFIC ${ }^{3}$ H]ETORPHINE BINDING

$\mathrm{EC}_{50}$ of $0.91 \mathrm{nM}$ in the presence of $150 \mathrm{mM}$ $\mathrm{NaCl}$. 
NIH 10810 (continued)

MOUSE VAS DEFERENS PREPARATION

\begin{tabular}{||c|c|c|c|c||}
\hline Condition & $\mathrm{EC}_{50}(\mathrm{nM})$ & $\begin{array}{l}\text { Maximum } \\
\text { Response (\%) }\end{array}$ & $\begin{array}{l}\text { Shift } \\
\text { (x-fold) }\end{array}$ & $\mathrm{n}$ \\
\hline \hline $\begin{array}{l}\text { Control } \\
\text { Naltrexone } \\
(100 \mathrm{nM})\end{array}$ & $60.4 \pm 4.7$ & $77.7 \pm 1.7$ & 6.9 & 3 \\
\hline $\begin{array}{l}\text { ICI } 174,864 \\
(100 \mathrm{nM})\end{array}$ & $8.00 \pm 0.93$ & $73.9 \pm 2.7$ & 0.9 & 3 \\
\hline $\begin{array}{l}\text { Nor-BNI } \\
(10 \mathrm{nM})\end{array}$ & $13.4 \pm 0.7$ & $73.9 \pm 2.7$ & 1.5 & 3 \\
\hline
\end{tabular}

\section{SUMMARY}

NIH 10810 had high affinity in the binding assay and, in the mouse vas deferens preparation, acted as an agonist relatively selective for $\mu$ opioid receptors.

NIH 10811

N-Cyclopropylmethyl[7 $\alpha, 8 \alpha, 2$ ',3']cyclohexano-1'[S]hydroxy-6,14-endo ethenotetrahydronororipavine hydrochloride

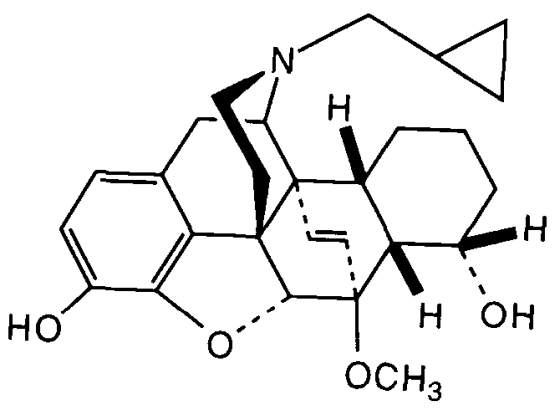

DISPLACEMENT OF SPECIFIC $\left[{ }^{3}\right.$ H]ETORPHINE BINDING

$\mathrm{EC}_{50}$ of $0.59 \mathrm{nM}$ in the presence of $150 \mathrm{mM} \mathrm{NaCl}$. 
NIH 10811 (continued)

MOUSE VAS DEFERENS PREPARATION

\begin{tabular}{||c|c|c|c|c||}
\hline \hline Condition & $\mathrm{EC}_{50}(\mathrm{nM})$ & $\begin{array}{l}\text { Maximum } \\
\text { Response }(\%)\end{array}$ & $\begin{array}{l}\text { Shift } \\
\text { (x-fold) }\end{array}$ & $\mathrm{n}$ \\
\hline \hline $\begin{array}{l}\text { Control } \\
(100 \mathrm{nM})\end{array}$ & $0.86 \pm 0.06$ & $99.1 \pm 0.9$ & 2.7 & 3 \\
\hline $\begin{array}{l}\text { ICI } 174864 \\
(100 \mathrm{nM})\end{array}$ & $1.89 \pm 96.3$ & $96.3 \pm 1.7$ & 2.2 & 3 \\
\hline $\begin{array}{l}\text { Nor-BNI } \\
(10 \mathrm{nM})\end{array}$ & 1.15 & 100 & 1.3 & 3 \\
\hline \hline
\end{tabular}

\section{SUMMARY}

NIH 10811 had high affinity in the binding assay and, in the mouse vas deferens preparation, acted as an agonist, perhaps, relatively selective for $\delta$ opioid receptors.

NIH 10812 2-Nitrobuprenorphine hydrochloride

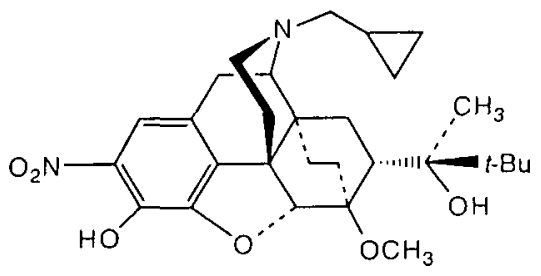

D I S P L A C E M E N T O F SPECIFIC [ $\left.{ }^{3} \mathrm{H}\right] \mathrm{ETOR}-$ PHINE BINDING

$\mathrm{EC}_{50}$ of $3805 \mathrm{nM}$ in the presence of $150 \mathrm{mM} \mathrm{NaCl}$.

MOUSE VAS DEFERENS PREPARATION

\begin{tabular}{||l|c|c|c|c||}
\hline Condition & $\mathrm{EC}_{50}(\mathrm{nM})$ & $\begin{array}{l}\text { Maximum } \\
\text { Response }(\%)\end{array}$ & $\begin{array}{l}\text { Shift } \\
\text { (x-fold) }\end{array}$ & $\mathrm{n}$ \\
\hline \hline Control & $6.65 \pm 1.69$ & $55.9 \pm 3.7$ & & 3 \\
\hline $\begin{array}{l}\text { Naltrexone } \\
(100 \mathrm{nM})\end{array}$ & $1.19 \pm 5.58$ & $40.0 \pm 1.7$ & 1.2 & 3 \\
\hline \hline
\end{tabular}


NIH 10812 (continued)

\begin{tabular}{||c|c|c|c|c|}
\hline Agonist & $\mathrm{pA}_{2}$ & Slope \pm S.E. & $\begin{array}{l}\mathrm{pA}_{2} \text { (Constrained) } \\
\pm \text { S.E. }\end{array}$ & $\mathrm{n}$ \\
\hline $\mathrm{DSLET}(\boldsymbol{8})$ & 5.31 & $1.34 \pm 0.04$ & $5.35 \pm 1.04$ & 3 \\
\hline $\mathrm{U} 50,488(\mathbf{k})$ & 5.90 & $1.02 \pm 0.16$ & $5.92 \pm 0.78$ & 3 \\
\hline \hline
\end{tabular}

\section{SUMMARY}

$\mathrm{NIH} 10812$ had low affinity in the binding assay. In concentrations of $0.1 \mathrm{nM}$ to $100 \mu \mathrm{M}$, it decreased the magnitude of the twitch of the electrically-stimulated mouse vas deferens preparation. The NIH 10812 concentration-effect curve was not shifted by naltrexone, although the maximum response was slightly decreased. NIH 10812 was a very weak antagonist at $\mu, \delta$, and $\kappa$ receptors. In the presence of NIH 10812, $10 \mu \mathrm{M}$, there was a 2.8 -fold shift to the right in the sufentanil concentration-effect curve. This drug was slightly more potent as an antagonist at $\boldsymbol{\kappa}$ and $\delta$ opioid receptors. ${ }_{*} \quad *$

NIH 10813

2-Nitrodiprenorphine hydrochloride

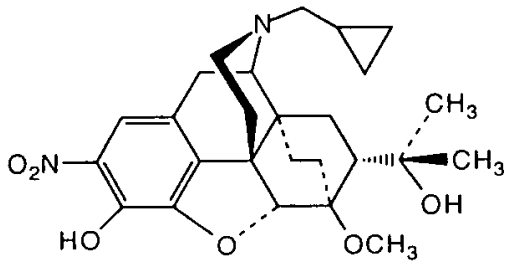

DISPLACEMENT OF SPECIFIC $\left[{ }^{3}\right.$ H]ETORPHINE BINDING

$\mathrm{EC}_{50}$ of $140 \mathrm{nM}$ in the presence of $150 \mathrm{mM} \mathrm{NaCl}$.

MOUSE VAS DEFERENS PREPARATION

\begin{tabular}{||l|c|c|c|c||}
\hline \hline Condition & $\mathrm{EC}_{50}(\mathrm{nM})$ & $\begin{array}{l}\text { Maximum } \\
\text { Response (\%) }\end{array}$ & $\begin{array}{l}\text { Shift } \\
(\mathrm{x} \text {-fold })\end{array}$ & $\mathrm{n}$ \\
\hline \hline Control & $412.7 \pm 29.0$ & $44.3 \pm 4.2$ & & 3 \\
\hline $\begin{array}{l}\text { Naltrexone } \\
(100 \mathrm{nM})\end{array}$ & $1865.1 \pm 84.8$ & $32.5 \pm 4.4$ & 4.5 & 3 \\
\hline
\end{tabular}


NIH 10813 (continued)

\begin{tabular}{||c|c|c|c|c||}
\hline Agonist & $\mathrm{pA}_{2}$ & Slope \pm S.E. & $\begin{array}{c}\mathrm{pA}_{2} \text { (Constrained) } \\
\pm \text { S.E. }\end{array}$ & $\mathrm{n}$ \\
\hline \hline Sufentanil $(\mu)$ & 7.47 & $1.01 \pm 0.06$ & $7.49 \pm 0.34$ & 6 \\
\hline DSLGT $(\delta)$ & 6.24 & $1.20 \pm 0.19$ & $6.39 \pm 0.4 \mathrm{I}$ & 6 \\
\hline U50,488 (K) & \multicolumn{3}{|c|}{ See below } \\
\hline
\end{tabular}

\section{SUMMARY}

NIH 10813 had moderate affinity in the binding assay. In concentrations of 100 $\mathrm{nM}$ to $30 \mu \mathrm{M}$, it decreased the magnitude of the twitch of the electricallystimulated mouse vas deferens preparation. Naltrexone, $100 \mathrm{nM}$, shifted the $\mathrm{NIH} 10813$ concentration effect curve to the right and decreased the maximum response. NIH 10813 was an antagonist at $\mu, \delta$, and $\kappa$ receptors. The antagonism of U50,488 was unsurmountable and began at a concentration of 1 $\mu \mathrm{M}$. The antagonism of DSLET and sufentanil appear to be competitive, and NIH 10813 was more potent as an antagonist at $\mathrm{k}$ receptors than at $\delta$ opioid receptors.

\section{NIH 10814 2-Nitronaltrexone hydrochloride

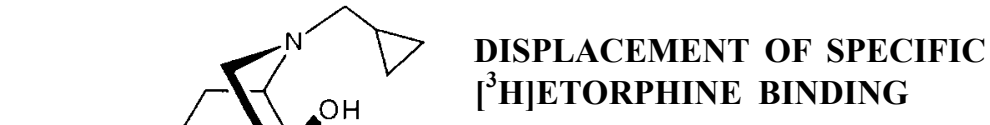

$\mathrm{EC}_{50}$ of $196 \mathrm{nM}$ in the presence of $150 \mathrm{mM}$ $\mathrm{NaCl}$.

MOUSE VAS DEFERENS PREPARATION

\begin{tabular}{||l|c|l|l|c||}
\hline Condition & $\mathrm{EC}_{50}(\mathrm{nM})$ & $\begin{array}{l}\text { Maximum } \\
\text { Response (\%) }\end{array}$ & $\begin{array}{l}\text { Shift } \\
\text { (x-fold) }\end{array}$ & $\mathrm{n}$ \\
\hline \hline Control & $3.48 \pm 1.14$ & $22.9 \pm 4.4$ & & 3 \\
\hline $\begin{array}{l}\text { Naltrexone } \\
(100 \mathrm{nM})\end{array}$ & $6.73 \pm 1.49$ & $24.7 \pm 2.4$ & 19 & 3 \\
\hline
\end{tabular}


NIH 10814 (continued)

\begin{tabular}{|c|c|c|c|c|}
\hline Agonist & $\mathrm{pA}_{2}$ & Slope \pm S.D. & $\begin{array}{l}\mathrm{pA}_{2} \quad(\text { Constrained }) \\
\pm \text { S.E. }\end{array}$ & $\mathrm{n}$ \\
\hline Sufentanil $(\mu)$ & 7.43 & $0.88 \pm 0.27$ & $7.31 \pm 0.34$ & 6 \\
\hline DSLET $(\delta)$ & 6.03 & $1.27 \pm 0.14$ & $6.18 \pm 0.43$ & 6 \\
\hline $\mathrm{U} 50,488$ (א) & \multicolumn{4}{|c|}{ See below } \\
\hline
\end{tabular}

\section{SUMMARY}

NIH 10814 had moderate affinity in the binding assay. In concentrations of $1 \mathrm{nM}$ to $100 \mu \mathrm{M}$, it decreased the magnitude of the twitch of the electrically-stimulated mouse vas deferens preparation. Naltrexone, $100 \mathrm{nM}$, shifted the NIH 10814 concentration effect curve to the right but did not decrease the maximum response. NIH 10814 was an antagonist at $\mu, \delta$, and $\kappa$ receptors. The antagonism of U50,488 was unsurmountable and began at a concentration of 1 $\mu \mathrm{M}$. The antagonism of DSLET and sufentanil appear to be competitive, and $\mathrm{NIH} 10814$ was more potent as an antagonist at $\mu$ receptors than at $\delta$ opioid receptors.

NIH 10815

(+)-4-[( $\alpha R)-\alpha(12 S, 5 R)-4-A l l y l-2,5-d i m e t h y l-1-$ piperazinyl)-3-methoxybenzyl]-N,Ndiethylbenzamide

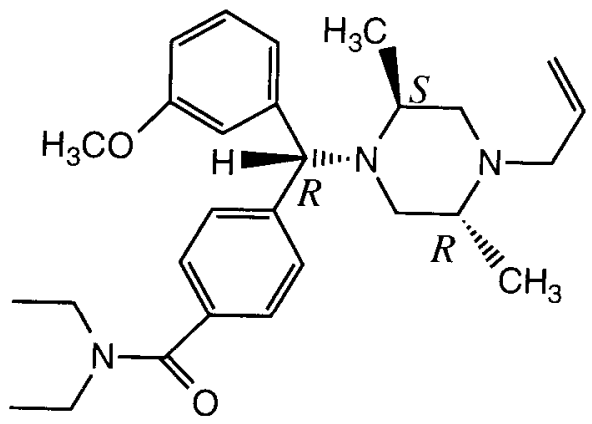

\section{DISPLACEMENT OF SPECIFIC $\left[{ }^{3}\right.$ H]ETORPHINE BINDING}

$\mathrm{EC}_{50}$ of $>6,000 \mathrm{nM}(43 \%$ inhibition at $6 \mu \mathrm{M})$ in the presence of $150 \mathrm{mM} \mathrm{NaCl}$. 
NIH 10815 (continued)

MOUSE VAS DEFERENS PREPARATION

\begin{tabular}{||l|c|c|c|c||}
\hline \hline Condition & $\mathrm{EC}_{50}(\mathrm{nM})$ & $\begin{array}{l}\text { Maximum } \\
\text { Response (\%) }\end{array}$ & $\begin{array}{l}\text { Shift } \\
\text { (x-fold) }\end{array}$ & $\mathrm{n}$ \\
\hline \hline Control & $6.38 \pm 0.58$ & 100 & & 9 \\
\hline $\begin{array}{l}\text { Nalfrexone } \\
(100 \mathrm{nM})\end{array}$ & $25.0 \pm 8.8$ & 100 & 3.9 & 3 \\
\hline $\begin{array}{c}\mathrm{ICI} 174,864 \\
(100 \mathrm{nM})\end{array}$ & $18.9 \pm 2.6$ & 100 & 3.0 & 3 \\
\hline $\begin{array}{c}\text { Nor-BNI } \\
(10 \mathrm{nM})\end{array}$ & $11.4 \pm 0.46$ & 100 & 1.8 & 3 \\
\hline
\end{tabular}

\section{SUMMARY}

NIH 10815 had no significant activity in the binding assay and, in the mouse vas deferens preparation, acted as an agonist relatively selective for $\delta$ opioid receptors. It should be noted that the results of the two preparations are discordant.

\section{REFERENCES}

Bertalmio, A.J.; Herling, S.; Hampton, R.Y.; Winger, G.; and Woods, J.H. A procedure for rapid evaluation of the discriminative stimulus effects of drugs. $\boldsymbol{J}$ Pharmacol Meth 7:289-299, 1982.

Carter, B.D. and Medzihradsky, F. Opioid signal transduction in intact and fragmented SH-SY5Y neural cells. J Neurochem 58:1611-1619, 1992.

Clark, M.J., Carter, B.D. and Medzihradsky, F. Selectivity of ligand binding to opioid receptors in brain membranes from the rat, monkey and guinea pig. Eur J Pharmacol 148:343-351, 1988.

Clark, M.J. and Medzihradsky, F. Coupling of multiple opioid receptors to GTPase following selective receptor alkylation in brain membranes. Neuropharmacol 26:1763-1770, 1987.

Dykstra, L.A. and Woods, J.H. A tail withdrawal procedure for assessing analgesic activity in rhesus monkeys, J Pharmacol Meth 15:263-269, 1986. 
France, C.P. and Woods, J.H. Discriminative stimulus effects of naltrexone in morphine-treated rhesus monkeys. J Pharmacol Exp Ther 250:937-943, 1989.

France, C.P., de Costa, B.R., Jacobson, A.E., Rice, K.C. and Woods, J.H. Apparent affinity of opioid antagonists in morphine-treated rhesus monkeys discriminating between saline and naltrexone. J Pharmacol Exp Ther 252:600604, 1990.

France, C.P. and Woods, J.H. Respiratory effects of receptor-selective opioids in rhesus monkeys. In: Quirion, R., Jhamandas, K. and Gianoulakis, C. (Eds.), Progress in Clinical and Biological Research: The International Narcotics Research Conference (INRC) '89, Vol. 328. Alan R. Liss, Inc.: New York, pp. 295-298, 1990.

Howell, L.L., Bergman, J. and Morse, W.H. Effects of levorphanol and several K-selective opioids on respiration and behavior in rhesus monkeys. J Pharmacol Exp Ther 245:1364-372, 1988.

Medzihradsky, F. Novel biochemical determinants in the preclinical evaluation of opiates. NIDA Res Monogr 76:349-355, 1987.

Medzihradsky, F.; Emmerson, P.J; and Mousigian, C.A. Lipophilicity of opioids determined by a novel micromethod. J Pharmacol Meth.

Medzihradsky, F.; Dahlstrom, P.J.; Woods, J.H.; Fischel, S.V.; and Mitsos, S.E. Resolution in the receptor binding of putative $\mu$ and $\kappa$ opiates. Life Sci 34:2129-2138, 1984.

Perrine, T.D.; Atwell, L.; Tice, I.B.; Jacobson, A.E.; and May, E.L. Analgesic activity as determined by the Nilsen method. J Pharm Sci 61:86-88, 1972.

Solomon, R.E.; Herling, S.; Domino, E.F.; and Woods, J.H. Discriminative stimulus effects of N-substituted analogs of phencyclidine in rhesus monkeys, Neuropharmacol 21:1329-1336, 1982.

Smith, C.B. New approaches to the evaluation of opioid agonists and antagonists upon the mouse vas deferens preparation, NIDA Res Monogr 76:288-294, 1986.

Smith, C.B.; Medzihradsky, F.; Hollingsworth, P.J.; DeCosta, B.; Rice, K.C.; and Woods, J.H. Nor-binaltorphimine is a reversible, noncompetitive opioid antagonist in the mouse vas deferens with high affinity for $\boldsymbol{k}$ receptors in monkey brain membranes. In: Quirion, R.; Jhamandas, K.; and Gianoulakis, C. eds., The International Narcotics Research Conference (INRC) '89. A.R. Liss, Inc., pp. 65-68, 1989. 
Winger, G.; Palmer, R.K.; and Woods, J.H.: Drug-reinforced responding: rapid determination of dose-response fimctions. Drug and Alc Depend 24:135-142, 1989.

Woods, J.H.; Smith, C.B; Medzihradsky, F.; and Swain, H.H. Preclinical testing of new analgesic drugs. In: Beers, F.R., Jr. and Basset, E.G. eds. Mechanisms of Pain and Analgesic Compounds. New York: Raven Press, pp. 429-445, 1979.

\section{AFFILIATION}

The Drug Abuse Basic Research Program, Departments of Pharmacology, Psychology, and Biological Chemistry, University Of Michigan, Ann Arbor, MI 


\title{
DEPENDENCE STUDIES OF NEW COMPOUNDS IN THE RHESUS MONKEY, RAT AND MOUSE (1994)
}

\author{
M.D. Aceto, E.R. Bowman, L.S. Harris and E.L. May
}

All compounds, except dextrorphan tartrate were supplied by Dr. Arthur Jacobson, Laboratory of Medicinal Chemistry, NIDDK, NIH. The identities of all the compounds, except that indicated above, were unknown to us when they were originally submitted. These studies were conducted under the auspices of the Drug Evaluation Committee of the College on Problems of Drug Dependence.

\section{Dependence-Liability Studies in Rhesus Monkeys}

Substitution-for-Morphine (SDS) Test. Male and female rhesus monkeys (M. mulatta) weighing $2.5-7.5 \mathrm{~kg}$ were used, and they received $3 \mathrm{mg} / \mathrm{kg}$, s.c., of morphiney $\cdot \mathrm{SO}_{4}$ every $6 \mathrm{~h}$. All the animals had received morphine for at least 3 months and were maximally dependent on morphine (Seevers and Deneau 1963). A minimal 2-week recuperation period was allowed between tests. At least 3 monkeys/dose were used. The assay (Aceto and co-workers, 1977 and 1978) was initiated by a subcutaneous injection of the test drug or control substances (morphine and vehicle) into animals in a group that had not received morphine for 14-15 h and showed definite signs of withdrawal. Each animal was randomly chosen to receive one of the following treatments: a) a dose of the compound under investigation; b) morphine control, $3.0 \mathrm{mg} / \mathrm{kg}$; and c) vehicle control, $1 \mathrm{ml} / \mathrm{kg}$. The animals were scored for suppression of withdrawal signs during a 2.5-h observation period. The observer was "blind" regarding the choice of treatments. At the end of the study, the data were grouped according to dose and drug. The mean cumulative score \pm SEM was calculated and the data illustrated in figure form. If indicated, the data were analysed using the Kruskal-Wallis Anova and posthoc Mann-Whitney U-Tests.

Precipitated-Withdrawal (PPT-W) Test. This evaluation was done under the same conditions as described above, except that the animals were administered a test compound $2-3 \mathrm{~h}$ after the last dose of morphine. These animals were not in withdrawal. Naloxone $\cdot \mathrm{HCl}(0.05 \mathrm{mg} / \mathrm{kg}$, s.c. $)$ served as the positive control.

Primary-Physical-Dependence (PPD) Study. Drug-naive monkeys were medicated with drug, using escalating dose regimens, periodically challenged with naloxone or placed in abrupt withdrawal. They were observed for overt behavioral signs during drug administration and when they were challenged with antagonist or abruptly withdrawn from the drug.

\section{Rat-Infusion Studies}

The continuous-infusion method was reported by Teiger (1974) and certain modifications are indicated as follows. Rats were anesthetized after which each was fitted with a specially prepared cannula which was passed subcutaneously from the nape of the neck to the lateral side of the lower abdomen and then 
inserted into the peritoneal cavity. The cannula was anchored at both ends with silk sutures and attached to a flow-through swivel mechanism which allowed the animal to move about in the cage and eat and drink normally. The swivel was connected to a syringe which was attached to a syringe pump. The animals received 7-10 $\mathrm{ml}$ of solution every $24 \mathrm{~h}$. Occasionally, when deemed necessary, as with cocaine, infusions were given via the right jugular vein.

Substitution-for-Morphine (SM) Test. The rats received morphine $\cdot \mathrm{SO}_{4}(50$ $\mathrm{mg} / \mathrm{kg} / 24 \mathrm{~h}$ on the first day, $100 \mathrm{mg} / \mathrm{kg} / 24 \mathrm{~h}$ on the second day, and 200 $\mathrm{mg} / \mathrm{kg} / 24 \mathrm{~h}$ from days 3 and 4). Then, a test drug was substituted for 2 days. The morphine controls received an infusion of water. The animals were observed for changes in body weight and for behavioral-withdrawal signs for $0.5 \mathrm{~h}$ at $6,24,48,72 \mathrm{and} /$ or $96 \mathrm{~h}$ after stopping the infusion of morphine.

Primary-Physical-Dependence (PPD) Study. The rats received test compound, as specified above, for 6 days and then, were placed in abrupt withdrawal and observed for overt behavioral signs.

\section{Mouse-Antinociception Tests}

Male mice, weighing 20-30 g, were used. All drugs were dissolved in distilled water or in the vehicle indicated and injected subcutaneously (s.c.). At least three doses were tested, and 6-10 animals per dose were used. When applicable, ED50's were calculated by using computerized probit analysis.

Tail-Flick (TF) and (TF vs M) Assays. The procedure and modifications were described (D'Amour and Smith, 1941 and Dewey et al., 1970 and 1971) in the literature. Briefly, the mouse's tail was placed in a groove which contained a slit under which was located a photoelectric cell. When the heat source of noxious stimulus was turned on, the heat focused on the tail, and the animal responded by flicking its tail out of the groove. Thus, light passed through the slit and activated the photocell which, in turn, stopped the recording timer. The heat source was adjusted to produce tail flick of 2-4 s under control conditions. Mice were injected with drug or vehicle and tested $20 \mathrm{~m}$ later. In the assay for antagonism of the antinociceptive effect, the potential antagonists were administered $10 \mathrm{~m}$ before the agonist, and evaluation occurred $20 \mathrm{~m}$ later.

Phenylquinone Abdominal-Stretching (PPQ) Assay. The procedure was reported previously (Pearl and Harris, 1966). The mice were injected with test drugs and $10 \mathrm{~m}$ later received $2.0 \mathrm{mg} / \mathrm{kg}$ ip of a freshly prepared paraphenylquinone (PPQ) solution. The mice were then placed in cages in groups of two each. Ten minutes after the PPQ injection, the total number of stretches per group were counted over a $1-\mathrm{m}$ period. A stretch was characterized by an elongation of the mouse's body, development of tension in the abdominal muscles, and extension of the forelimbs. The antinociceptive response was expressed as the percent inhibition of the PPQ-induced stretching response.

Hot-Plate (HP) Assay. The method was also reported previously (Eddy and Leimbach, 1953 and Atwell and Jacobson, 1978). The hot plate was held at $55^{\circ} \mathrm{C}$. Mice were placed on the hot plate and activity was scored if the animal jumped or licked its paws after a delay of $5 \mathrm{~s}$ or more, but no more than $30 \mathrm{~s}$ beyond the control time. Table 2 contains a summary of all the new data generated this year on compounds that had not previously been tested. 
Comparative Data (ED50, $\mathrm{mg} / \mathrm{kg}$ s.c.) [95\% C.L.] of Selected Standards in 4 Mouse Agonist-Antagonist Tests

\begin{tabular}{|c|c|c|c|c|}
\hline Drug & Tail-Flick & Tail-Flick & Phenylquinone & Hot-Plate \\
\hline & & Antagonist & & \\
\hline Pentazocine & $15 \%$ at 10.0 & $\begin{array}{c}18 \\
(12-26)\end{array}$ & $\begin{array}{c}1.7 \\
(1.0-2.5)\end{array}$ & --- \\
\hline Cyclazocine & $17 \%$ at $1.0^{\mathrm{a}}$ & $\begin{array}{c}0.03 \\
(0.020-0.78)\end{array}$ & $\begin{array}{c}0.01 \\
(0.005-0.03)\end{array}$ & --- \\
\hline Nalorphine $\cdot \mathrm{HCl}$ & None at 10.0 & $\begin{array}{c}2.6 \\
(0.7-10.0)\end{array}$ & $\begin{array}{c}0.6 \\
(0.03-1.44)\end{array}$ & --- \\
\hline Naloxone $\cdot \mathrm{HCl}$ & None at 10.0 & $\begin{array}{c}0.04 \\
(0.01-0.09)\end{array}$ & No Activity & --- \\
\hline Naltrexone $\cdot \mathrm{HCl}$ & None at 10.0 & $\begin{array}{c}0.007 \\
(.002-0.02)\end{array}$ & No Activity & --- \\
\hline Morphine $\cdot \mathrm{SO}_{4}{ }^{\mathrm{b}}$ & $\begin{array}{c}0.7^{\mathrm{b}} \\
(0.4-1.5)\end{array}$ & Inactive & $\begin{array}{c}0.4^{\mathrm{b}} \\
(0.2-0.8)\end{array}$ & $\begin{array}{c}3.1^{\mathrm{b}} \\
(1.5-6.4)\end{array}$ \\
\hline Codeine $\cdot \mathrm{PO}_{4}$ & -- & Inactive & $\begin{array}{c}--- \\
(0.39-16.8)\end{array}$ & $\begin{array}{c}6.4 \\
(0.39-16.8)\end{array}$ \\
\hline Meperidine $\cdot \mathrm{HCl}$ & -- & Inactive & -- & $\begin{array}{c}4.6 \\
(1.8-11.7)\end{array}$ \\
\hline
\end{tabular}

${ }^{\mathrm{a}}$ Mice were ataxic at 3.0 and $10.0 \mathrm{mg} / \mathrm{kg}$ but there was no further increase in reaction time

${ }^{\mathrm{b}} \mathrm{ICR}$ - Harlan-Sprague-Dawley Inc.

Calculation of Apparent $p A_{2}$ Using the tail-flick assay, the apparent $\mathrm{pA}_{2}$ and $95 \%$ confidence limits were calculated using Schild and constrained plots as described in Tallarida and Murray (Manual of Pharmacologic Calculations with Computer Programs, 2nd ed., Springer Verlag, NY., 1987).

Briefly, mice were pretreated with vehicle or various doses of antagonist followed $10 \mathrm{~m}$ later by an injection of agonist. The mice were tested $30 \mathrm{~m}$ after receiving the antagonist. Dose-response lines for antinociception were plotted using at least 3 doses of each opioid agonist in the presence of vehicle or one of the selected doses of antagonist. ED50s were estimated according to the method of Litchfield and Wilcoxon (J. Pharmacol, Exp. Ther., 96, 399, 1949). Each dose ratio (x) was calculated by dividing the ED50 of the opioid in the presence of a given dose of antagonist by that of the agonist alone. Log (x-1) was plotted against the negative logarithm of the molar dose of the antagonist. At least 3 logs $(\mathrm{x}-1)$ were plotted. The $\mathrm{pA}_{2}$ values for the antagonist were calculated from the point of intersection of the regression line with the abscissa. See Table 2 for summary of results. 
Table 2. Apparent $\mathrm{pA}_{2}$ values $^{\mathrm{a}}$ using the mouse tail-flick assay

\begin{tabular}{|c|c|c|c|}
\hline \multicolumn{2}{|c|}{$\begin{array}{l}\text { Treatment } \\
\text { Antagonist/Agonist }\end{array}$} & $\mathrm{pA}_{2} \frac{\text { Schild PloT }}{(95 \% \text { C.L) }}$ Slope & $\begin{array}{l}\text { Constrained Plot } \\
\mathrm{pA}_{2}(95 \% \text { C.L })\end{array}$ \\
\hline 1) & Naloxone/Morphine & $7.2(7.0-7.4)-1.2$ & $7.3(7.1-7.6)$ \\
\hline 2) & Nalmefene/Morphine & $8.0(7.6-8.3)-1.1$ & $8.0(7.7-7.6)$ \\
\hline 3) & Naltrexone/Morphine & $7.7(4.9-10.5)-0.8$ & $7.6(7.1-8.3)$ \\
\hline 4) & (-)-Quadazocine/Morphine & $6.8(6.7-7.0)-0.9$ & $6.8(6.1-0 \quad 7.6)$ \\
\hline 5) & Naloxone/Sufentanil & $7.0(6.9-7.1)-1.0$ & $7.0(6.9-7.0)$ \\
\hline 6) & Naloxone/Sufentanil & $7.0(6.5-7.5)-1.0$ & $7.0(6.8-7.1)$ \\
\hline 7) & Naloxone/Mirfentanil & $7.6(7.3-8.0)-0.7$ & $7.2(6.9-7.5)$ \\
\hline 8) & Naloxone (-)-Nicotine & $5.3(5.3-5.3)-0.5$ & $7.0(6.9-7.0)$ \\
\hline 9) & Naloxone/U-50,488 & $6.6(6.3-6.9)-1.1$ & $7.2(6.9-7.5)$ \\
\hline & kappa agonist & & $6.6(6.3-7.0)$ \\
\hline 10) & $\begin{array}{l}\text { Naloxone/NIH } 10672 \\
\text { selective kappa agonist }\end{array}$ & $6.1(5.6-6.6)-1.2$ & $6.2(5.9-7.3)$ \\
\hline 11) & (-)-Quadazocine/NIH 10672 & $6.2(6.1-6.2)-1.7$ & $6.7(6.6-6.8)$ \\
\hline 12) & nor BNI/NIH 10672 & $6.5(5.9-7.0)-1.3$ & $6.6(5.9-7.3)$ \\
\hline 13) & Mecamylamine/(-)-Nicotine & $6.6(6.2-6.9)-0.9$ & $6.5(6.4-6.6)$ \\
\hline
\end{tabular}

${ }^{a}$ Negative logarithm of the molar concentrations of antagonist required to produce a two-fold shift of the agonist dose-response curve to the right. Competitive antagonism can be assumed when slope $=-1 . \mathrm{pA}_{2}$ provides a measure of the relative potency and affinity of the antagonist. When the slope differs significantly from unity, this may indicate non-equilibrium conditions, interactions with multireceptors, receptor sensitization, precoupling mechanisms, or multiple drug properties. With a constrained plot, the slope of the regression line is restricted to slope $=-1$. 
SUMMARY OF NEW DATA

\begin{tabular}{|c|c|c|c|c|c|c|c|c|c|c|c|c|}
\hline Compound & & Chemical Name & & & OUSE & & & & & & $\overline{1 O N K E Y}$ & \\
\hline $\mathrm{NIH}$ & & or Generic Class & TF & TFvsM & $P P Q$ & $\mathrm{HP}$ & PA2 & SM & PPD & SDS & PPt-W & PPD \\
\hline 04591 & Dextrorphan Tartrate & 3-Hydroxymorphan & & & & & & + & + & & & \\
\hline 10560 & & 6,7-Benzomorphan & & & & & $+a$ & & & & & \\
\hline 10675 & & 6,7-Benzomorphan & & & & & & + & + & & & \\
\hline 10683 & & Epoxymorphinan & $+{ }^{b c}$ & + & + & + & & & & + & & \\
\hline 10684 & & Epoxymorphinan & + & + & + & + & & & & + & + & \\
\hline 10735 & & 3-Hydroxymorphinan & + & + & + & + & & & & + & & \\
\hline 10738 & & 4-Phenylpiperidine & + & + & + & + & & & & + & & \\
\hline 10739 & & 4-Phenylpiperidine & + & + & + & + & & & & + & & \\
\hline 10741 & & 4-Amidopiperidine & & & & + & $++^{a}$ & & & & & \\
\hline 10749 & & 3-Hydroxymorphinan & + & + & + & + & & & & + & & \\
\hline 10773 & & Epoxymorphinan & + & + & + & + & & & & + & & \\
\hline 10779 & & Phenylmophan & + & + & + & + & & & & + & & \\
\hline 10782 & Rifampicin & Piperazine & + & + & + & + & & & & $+{ }^{d}$ & & \\
\hline 10784 & & 4-Amidopiperidine & $+{ }^{b}$ & + & + & + & & & & + & & \\
\hline 10785 & & 4-Amidopiperidine & $+{ }^{b}$ & + & + & + & & & & + & & \\
\hline 10786 & & 4-Amidopiperidine & $+{ }^{b}$ & + & + & + & & & & + & & \\
\hline 10787 & 1-Cloronaltrexone & Epoxymorphinan & $++^{e}$ & + & + & + & & & & + & + & \\
\hline 10790 & & 4-Amidopiperidine & $+b$ & + & + & + & & & & + & & \\
\hline 10791 & & 4-Amidopiperidine & $+{ }^{b}$ & + & + & + & & & & + & & \\
\hline 10792 & & 4-Amidopiperidine & + & + & + & + & & & & + & & \\
\hline 10793 & & 4-Amidopiperidine & $++^{b}$ & + & + & + & & & & + & & \\
\hline
\end{tabular}


SUMMARY OF NEW DATA

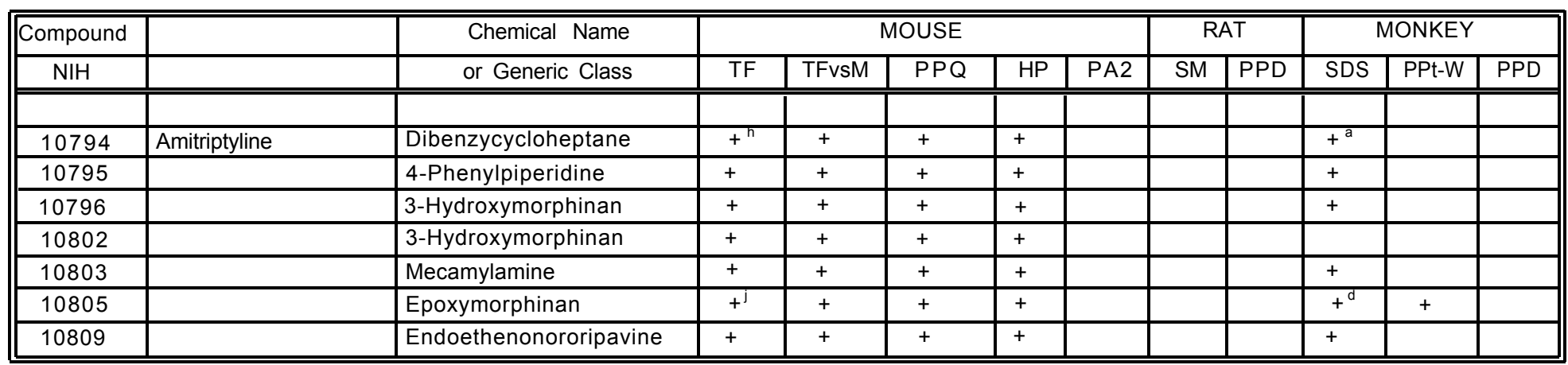

${ }^{a}$ Special $-\mathrm{pA}_{2}$ vs morphine in TF. ${ }^{\mathrm{b}}$ Special - Naloxone vs ED80 in TF. ${ }^{\mathrm{c}}$ Special - Naloxone vs ED80 in PPQ. ${ }^{\mathrm{d}}$ Special - Preliminary test in one

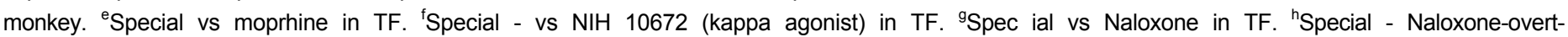
behavior-challenge test. 'Special - Naloxone challenge of overt neurological signs. 'Special - Time-course study vs morphine in TF. 


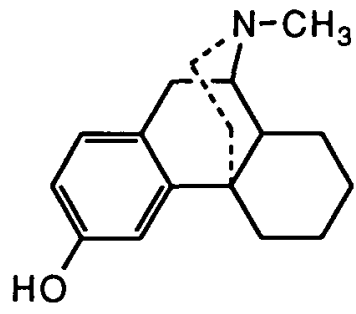

MOUSE DATA - ED50 OR AD50 $\mathrm{mg} / \mathrm{kg} \quad(95 \%$ C.L. $)$ or $\%$ change

1) $\mathrm{TF}$ - Inactive at $1.0,10.0$ and $30.0^{\mathrm{a}}$

2) $\mathrm{TF}$ vs. $\mathrm{M}-13 \%$ at $10.0,30 \%$ at $30.0^{\mathrm{a}}$ and $61 \%$ at 100.0

3) PPQ - $23.8(12.3-46.0)^{\mathrm{a}, \mathrm{b}}$

4) HP - Approx $30.0^{\mathrm{a}}$

${ }^{a}$ Published previously (NIDA Monog. 67, 1986)

${ }^{\mathrm{b}}$ At 0.1 and $1.0 \mathrm{mg} / \mathrm{kg}$ naloxone partly antagonized this effect

MONKEY - Monkey data reported previously in NIDA Monog. 67, 1986.

A. $\underline{\mathrm{SDS}}$

Dextrorphan reduced the total number of withdrawal signs but at $4.0 \mathrm{mg} / \mathrm{kg}$, it produced severe ataxia, slowing, body sag and partial eyelid ptosis. Dose range studied was $0.25-4.0 \mathrm{mg} / \mathrm{kg}$ s.c.

\section{B. $\quad \underline{\text { PPt-W }}$}

At doses of 1.0 and $4.0 \mathrm{mg} / \mathrm{kg}$ dextrorphan did not precipitate withdrawal. Dose-related side-effects as indicated above.

\section{C. $\quad \underline{P P D}$}

Given 4-6 times a day for 30 days at doses ranging from 3.0 to $13.0 \mathrm{mg} / \mathrm{kg}$, dextrorphan produced withdrawal phenomena. Tolerance to the acute effects developed rapidly up to a dose of $10.0 \mathrm{mg} / \mathrm{kg}$. When the monkeys were placed in abrupt withdrawal, a syndrome characterized by the signs lying on side or abdomen, scratching, wet-dog shakes, fighting, pacing, frequent touching of genital area, and rubbing face against pen wall was observed. When the monkeys were challenged with naltrexone $(1.0 \mathrm{mg} / \mathrm{kg}$ s.c.) withdrawal was exacerbated. However, none of the monkeys ever showed rigid abdominal muscles or vocalized when their abdomens were palpated. These signs are considered important and distinguished morphine withdrawal from dextrorphan withdrawal in the monkey.

\section{$\frac{\text { RAT DATA }}{\text { SM }}$ (New data)}

Unanesthetized, male, Sprague-Dawley rats previously fitted with intraperitoneal cannulas (Teiger, JPET, 1974) were made maximally dependent by continuously administered morphine using the following dose regimen: 50 $\mathrm{mg} / \mathrm{kg}$, day $1 ; 100 \mathrm{mg} / \mathrm{kg}$, day 2 and $200 \mathrm{mg} / \mathrm{kg}$, days 3 and 4 . At the end of day 4, morphine was abruptly withdrawn and dextrorphan (200 or $400 \mathrm{mg} / \mathrm{kg}$ per day) or vehicle ( $8 \mathrm{ml}$ per day) was substituted for morphine. A dose-related reduction in behavioral withdrawal signs was observed in rats receiving 
NM 04591 (+)-3-Hydroxy-N-methmylmorphinan tartrate or Dextrophan Tartrate (continued)

dextrorphan (see Fig. 1). The morphine-dependent rats given vehicle displayed the usual full complement of behavioral withdrawal signs. These behavioral signs were designated hypersensitivity, squeaking, aggression, wet-dog shakes, rubbing and chewing. None of the vehicle controls showed any withdrawal signs. However, the precipitous loss of body weight which occurred in the rats receiving morphine and vehicle or morphine and dextrorphan indicated that dextrorphan had no influence on this particular physiological withdrawal parameter (see Fig. 2). The results suggest that dextrorphan may selectively alter the behavioral expression of opioid dependence.

DEXTRORPHAN SM IN RAT INFUSION

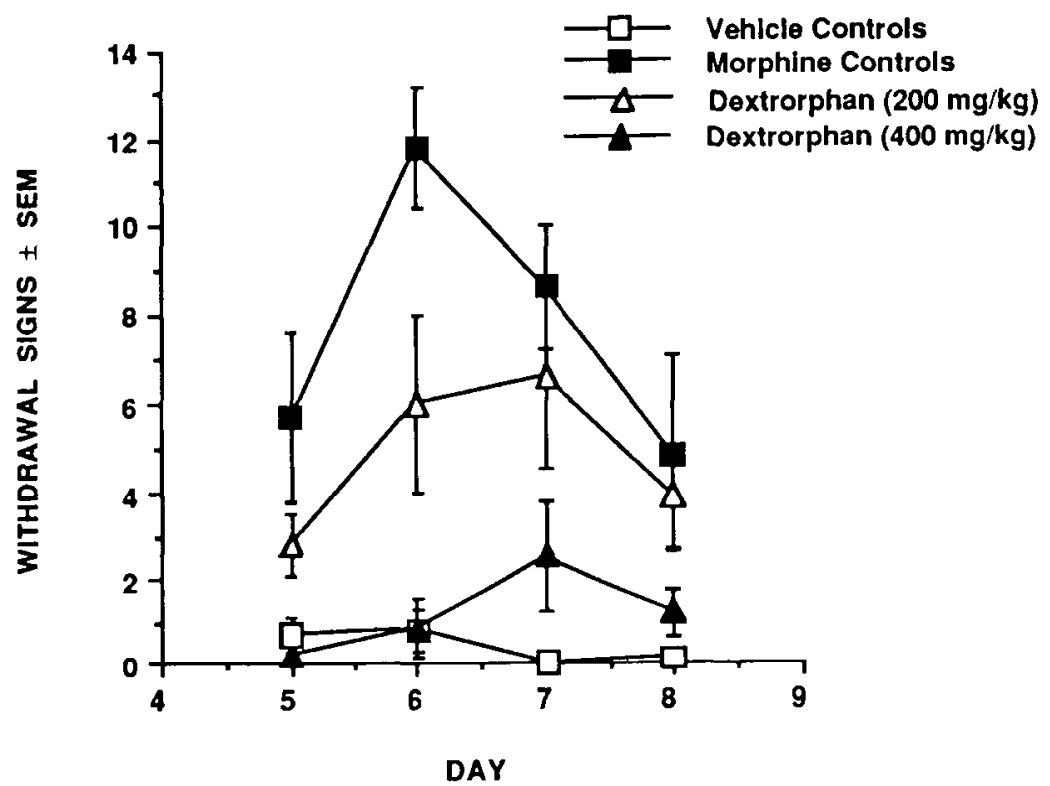


NIH $04591 \quad(+)-3-H y d r o x y-N-m e t h m y l m o r p h i n a n$ tartrate or Dextrophan Tartrate (continued)

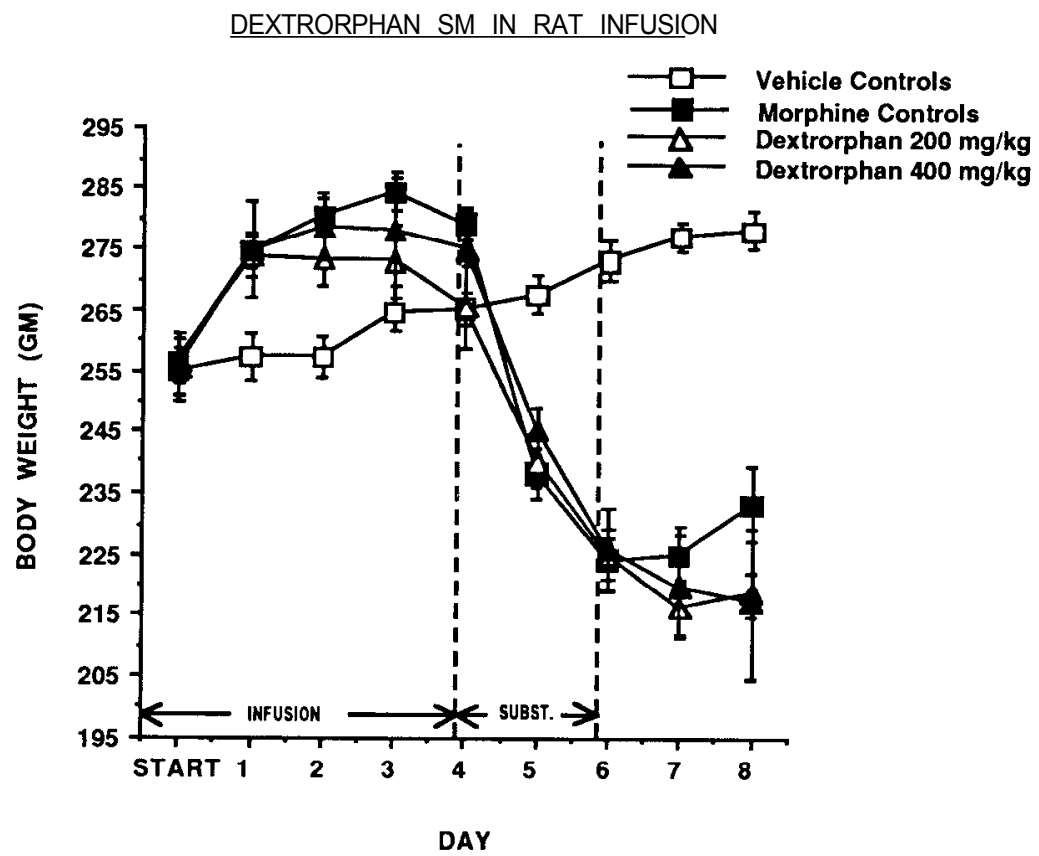

Comments:

Taken together, the results suggest that dextrorphan may interact indirectly with the opiate system, perhaps via the NMDA (N-Methyl-D-Aspartate) system.

NIH $10560 \quad(-)-5,9 \alpha$-Dimethyl-2-ethy1-2'hydroxy-6,7-benzomorphan hydrochloride

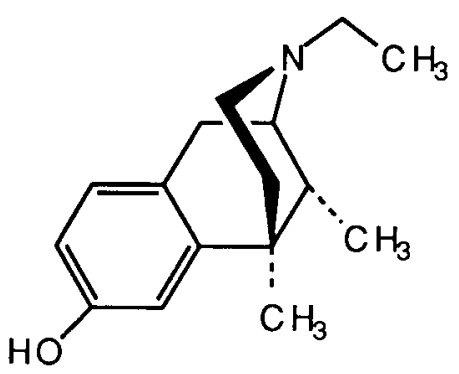

MOUSE DATA - ED50 OR AD50 $\mathrm{mg} / \mathrm{kg}(95 \%$ C.L.) or \% change

1) $\mathrm{TF}$ - Inactive at 1.0, 10.0 and $30.0^{\mathrm{a}}$

2) TF vs. M - $3.7(1.8-7.8)^{\mathrm{a}}$

3) PPQ - $1.4(0.5-4.1)^{\mathrm{a}}$

4) $\mathrm{HP}-13 \%$ at $1.0,25 \%$ at 10.0 and $30.0^{\mathrm{a}}$

${ }^{\mathrm{a}}$ Reported previously - NIDA Res Monog 90, 1988. 
NIH 10560 (-)-5,9 $\alpha$--Dimethyl-2-ethyl-2'hydroxy-6,7-benzomorphan hydrochloride (continued)

MOUSE DATA - New Data

pA 2 Assay 10560 vs morphine in TF.

NIH 10560 produced parallel shifts in the dose-response curve of morphine. The apparant $\mathrm{pA}_{2}$ and slope shown in the accompanying fig suggest that $\mathrm{NIH}$ 10560 has low affinity for mu receptors and that the interaction is competitive.

NIH 10560 VERSUS MORPHINE IN TAIL-FLICK Apparent pA2 Schild

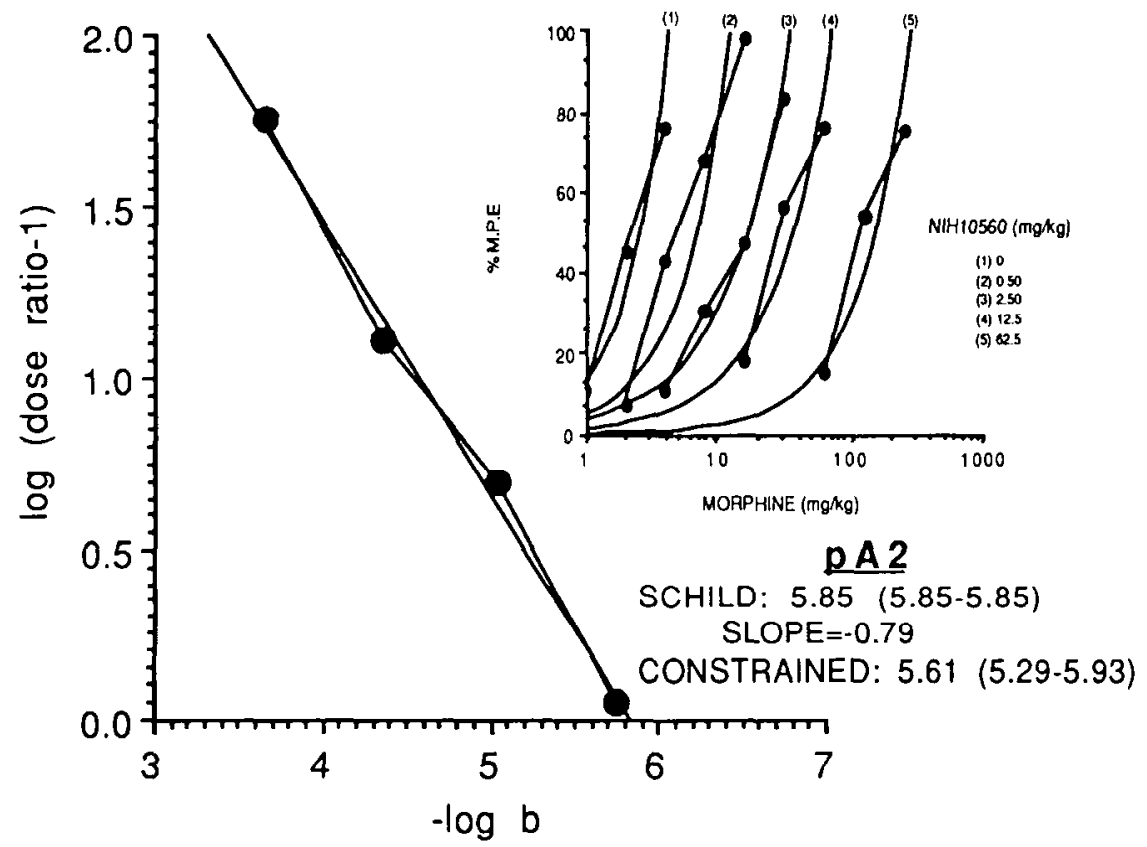

MONKEY DATA - Reported previously - op cit SDS

Did not substitute for morphine; instead, it exacerbated withdrawal 
NIH 10675 (-)-5,9 $\boldsymbol{\alpha}$-Dimethyl-2- $n$-heptyl-2'-hydroxyl-6,7-benzomorphan hydrochloride

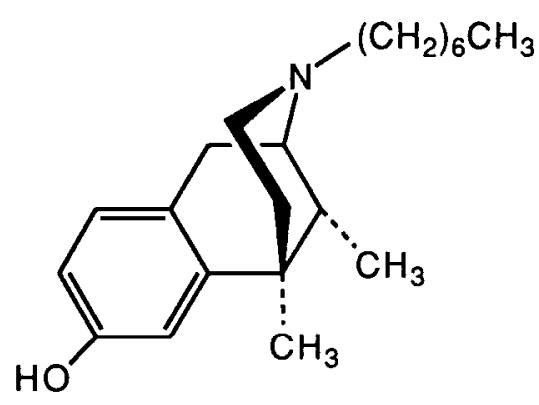

MOUSE DATA - ED50 OR AD50, $\mathrm{mg} / \mathrm{kg}$ (95\% C.L.) or \% change

1) $\mathrm{TF}-1.7(1.1-2.7)^{\mathrm{a}, \mathrm{b}}$

2) $\mathrm{TF}$ vs. $\mathrm{M}$ - Inactive at $1.0,10.0$ and $30.0^{\mathrm{a}, \mathrm{b}}$

3) PPQ $-0.13(0.002-0.99)^{\mathrm{a}, \mathrm{b}}$

4) $\mathrm{HP}-2.4(1.1-5.1)^{\mathrm{a}, \mathrm{c}}$

${ }^{a}$ Vehicle propylene glycol and water

${ }^{\mathrm{b}}$ Reported previously in NIDA Res.

Monog. 119, 1992

${ }^{\mathrm{c}}$ Reported in NIDA Res Monog. (in press, 1994)

MONKEY DATA

(SDS)

Reported previously op cit.

Neither substituted for morphine nor exacerbated withdrawal.

\section{$\underline{\text { RAT DATA - CONTINOUS INFUSION }}{ }^{\mathrm{a}}$ - New Data}

\section{A. $\underline{S M}$}

The results are summarized in the appropriate fig and table. NIH 10675 partly suppressed morphine-induced withdrawal in terms of body weight loss or with regard to suppression of withdrawal signs. 
NIH 10675 (-)-5,9 $\alpha$-Dimethyl-2- $n$-heptyl-2'-hydroxyl-6,7-benzomorphan hydrochloride (continued)

\section{NIH 10675 SM IN RATS}

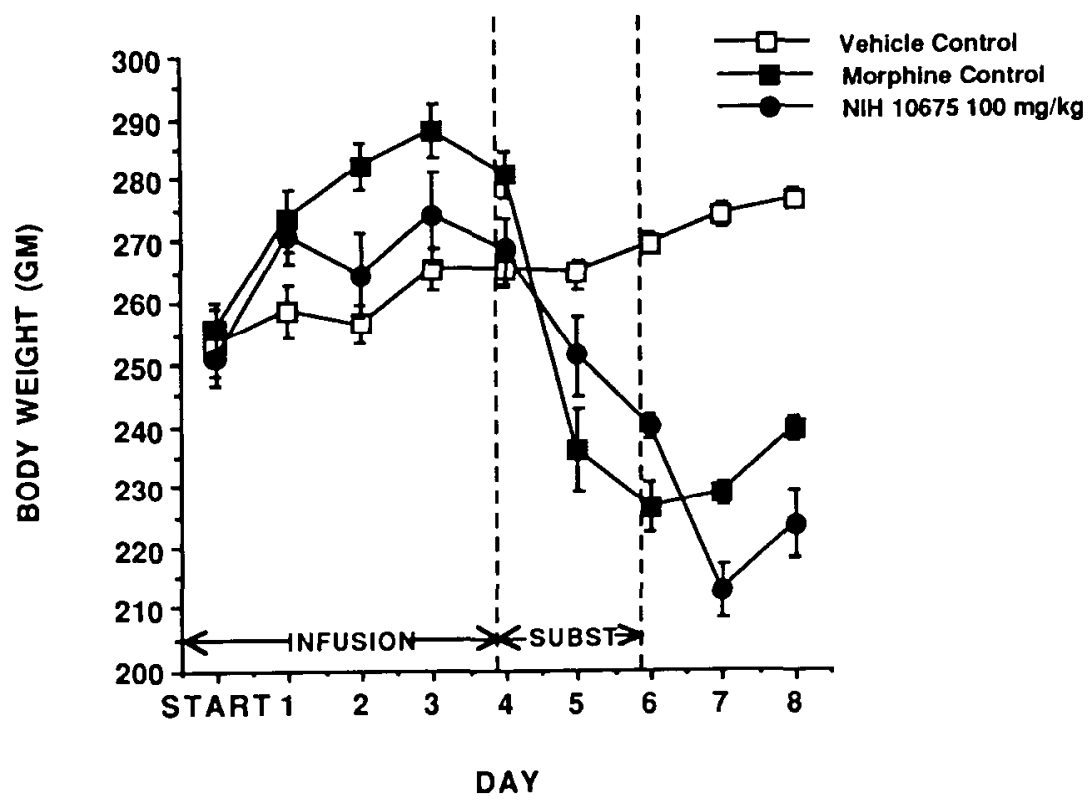

B. $\underline{P P D}^{\underline{b}}$

NIH 10675 produced a definite mu-like withdrawal syndrome when abruptly withdrawn after a 4-day infusion on a dose regimen similar to that of morphine.

a) A new dose regimen was used in this study. Instead of the usual 6-day regimen, we found that a 4-day regimen (see Table) was just as effecttve in producing physical dependence.

b) Three of the 6 rats appeared tranquilized.

\section{Comments:}

NM 10675 did not substitute for morphine in the monkey model. However, in both rodent models, it showed actions associated with mu-like agonists. Metabolic considerations may underlie the difference between the monkey and rodent models. 
Table: Primary Physical Dependence (PPD) and Substitution for Morphine Studies (SM) with NIH 10675 in Continuously-Infused Rats

$\underline{\text { Treatment }}$

\begin{tabular}{|c|c|c|c|}
\hline \multicolumn{4}{|c|}{$\mathrm{Hr}$ in Withdrawal } \\
\hline $24($ day 5$)$ & 48 (day 6$)$ & 72 (day 7$)$ & 96 (day 8$)$ \\
\hline
\end{tabular}

1. Vehicle Controls ${ }^{\mathrm{d}}$

0.8

1.8

0

0.3

2. Morphine Controls ${ }^{\mathrm{e}}$

8.4

$12.0^{\mathrm{b}}$

$9.2^{\mathrm{b}}$

8.0

3. NIH $10675-$ PPD $^{\mathrm{f}}$

$7.8^{\mathrm{b}}$

$4.3^{\mathrm{c}}$

$4.2^{\mathrm{b}, \mathrm{c}}$

0.7

4. NIH $10675-\mathrm{SM}^{\mathrm{g}}$

$3.4^{\mathrm{b}}$

$3.0^{\mathrm{c}}$

$13.6^{\mathrm{b}}$

7.8

${ }^{a}$ Hypersensitivity, squeaking, aggression, wet-dog shakes, rubbing and chewing;

${ }^{\mathrm{b}}$ One-tailed test Mann-Whitney-U test, $\mathrm{p}<0.05$, probability value vs. vehicle controls;

${ }^{\mathrm{c}}$ One-tailed test Mann-Whitney-U test, $\mathrm{p}<0.05$, probability value vs morphine controls;

d $8 \mathrm{ml} / 24 \mathrm{hr} . \mathrm{N}=4$;

${ }^{\mathrm{e}}$ Dose regimen of morphine, $\mathrm{SO}_{4}, 50 \mathrm{mg} / \mathrm{kg}$ on day $1,100 \mathrm{mg} / \mathrm{kg}$ on day $2,200 \mathrm{mg} / \mathrm{kg}$ on days 3 and $4 . \mathrm{N}=5$;

${ }^{\mathrm{f}}$ Dose regimen of $\mathrm{NIH} 10675,50 \mathrm{mg} / \mathrm{kg}$ on day $1,100 \mathrm{mgkg}$ on day $2,200 \mathrm{mg} / \mathrm{kg}$ on days 3 and 4 . N=6;

${ }^{\mathrm{g}}$ Morphine $\mathrm{SO}_{4}$ infusion, days $1-4$ as above then, $\mathrm{NIH} 10675100 \mathrm{mg} / \mathrm{kg}$ on days 5 and 6 , and vehicle as above on days 7 and $8 . \mathrm{N}=5$. 


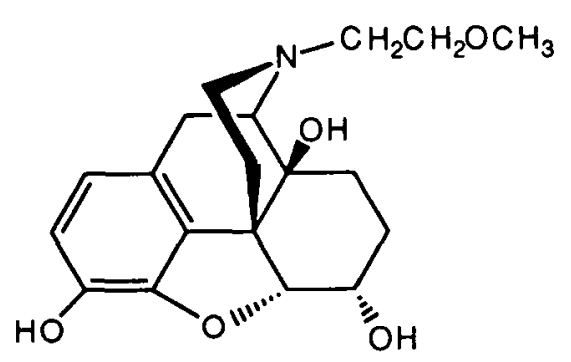

MOUSE DATA - ED50 OR

AD $50, \mathrm{mg} / \mathrm{kg}$

(95\% C.L.) or \% change

1) $\mathrm{TF}-0.2(0.1-0.4)^{\mathrm{a}}$

2) $\mathrm{TF}$ vs. $\mathrm{M}$ - Inactive at 1.0 , 10.0 and $30.0^{\mathrm{a}}$

3) PPQ - $0.03(0.01-0.09)^{\mathrm{a}}$

4) $\mathrm{HP}-0.3(0.1-0.9)^{\mathrm{a}}$

${ }^{\mathrm{a}}$ Vehicle -phosphoric acid and water

Special Tests

1) Naloxone vs ED80 of NIH 10683 in TF - AD50 =0.03 $(0.01-0.1)$

2) Naloxone vs ED80 of NIH 10683 in PPQ - AD50 = $0.6(0.1-1.7)$

\section{MONKEY DATA}

(SDS)

NIH 10683 niether substituted for morphine nor exacerbated withdrawal at doses of $0.5,2.0$ and $6.0 \mathrm{mg} / \mathrm{kg}$ (see graph). A non-dose related reduction in the signs designated retching and wet-dog shakes was noted. Vehicle was $\mathrm{H}_{3} \mathrm{PO}_{4}$ and $\mathrm{H}_{2} \mathrm{O}$.

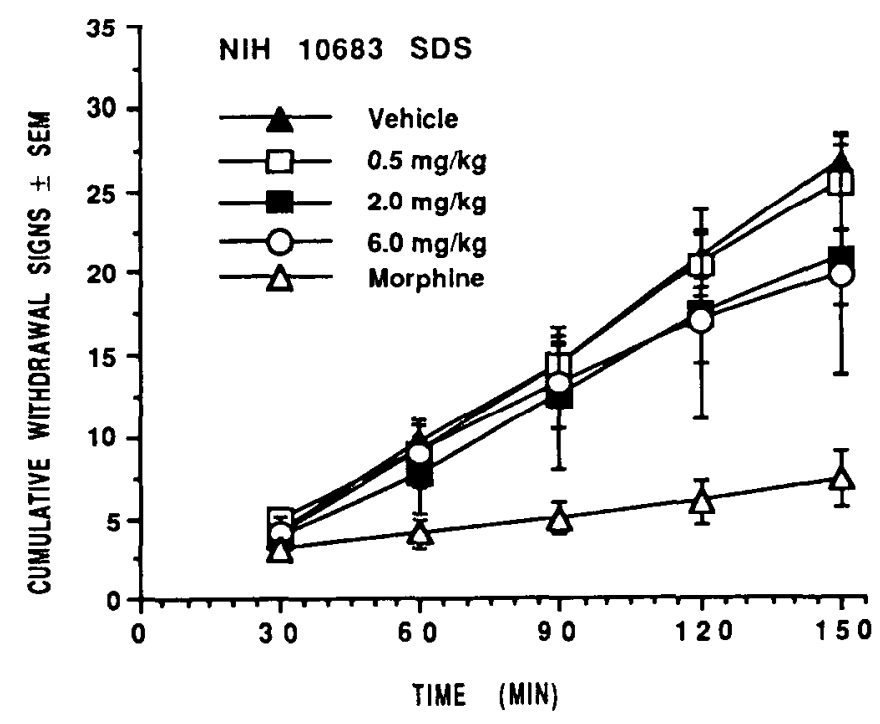

\section{Comments:}

NIH 10683 may have multiple (mu, kappa and/or delta) opioid properties in the mouse. In the monkey, the effects are not mu-like. 


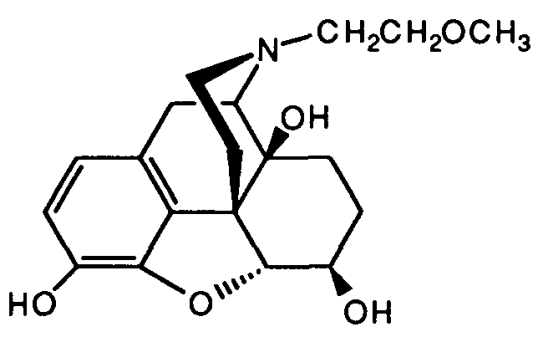

MOUSE DATA - ED50 OR AD50, $\mathrm{mg} / \mathrm{kg}$

( $95 \%$ C.L.) or $\%$ change

1) $\mathrm{TF}$ - Inactive at $1.0,10.0$ and $30.0^{\mathrm{a}}$

2) TFvs.M - $4 \%$ at $1.0,26 \%$ at 10.0 and $30.0^{\mathrm{a}}$

3) PPQ - $2.6(0.5-13.7)^{\mathrm{a}}$

4) $\mathrm{HP}-13 \%$ at $1.0,10.0$ and $30.0^{\mathrm{a}}$

${ }^{a}$ Vehicle -phosphoric acid and water

\section{MONKEY DATA}

A. (SDS)

As shown in the accompanying illustration, this compound did not substitute for morphine. Instead, it appeared to exacerbate withdrawal in a dose-related manner. One monkey was still showing withdrawal signs at 6:00 p.m. in spite of the fact that the dose of morphine was doubled. Some of the animals were very aggressive towards the handler. Vehicle was phosphoric acid and water.

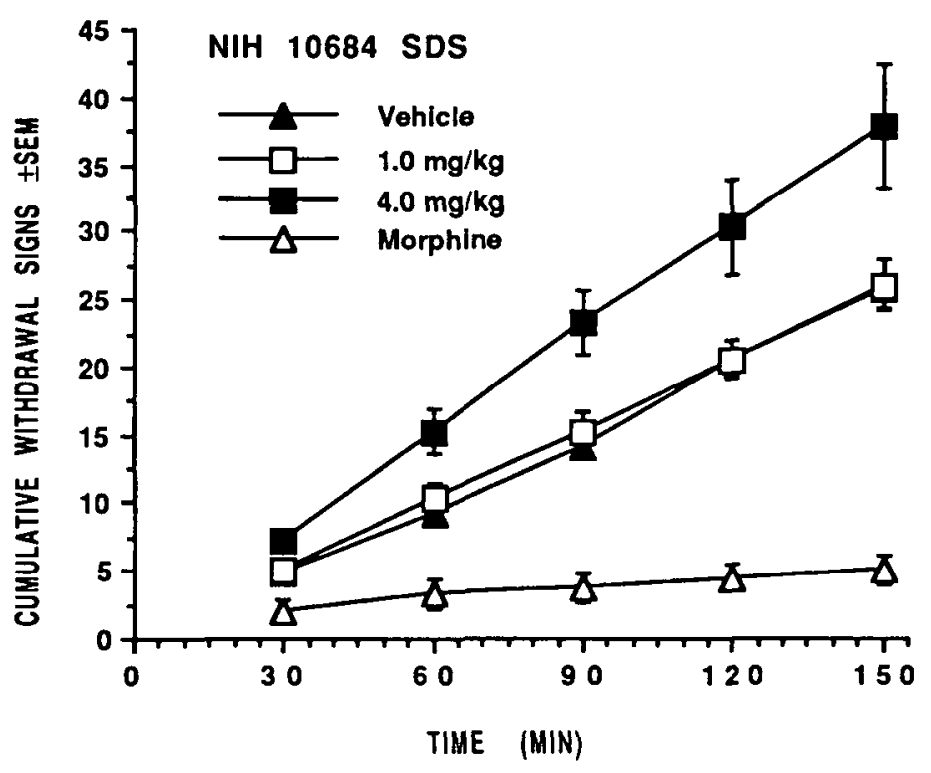


NIH 10684 14-Hydroxy-N-(2-methoxyethyl)-7,8-dihydronorisomorphine (continued)

B. (PPt-W)

NIH 10684 precipitated withdrawal and the actin was dose-related. While naloxone had a quick onset and short duration of action, this compound had a slower onset and long duration of action (see graph).

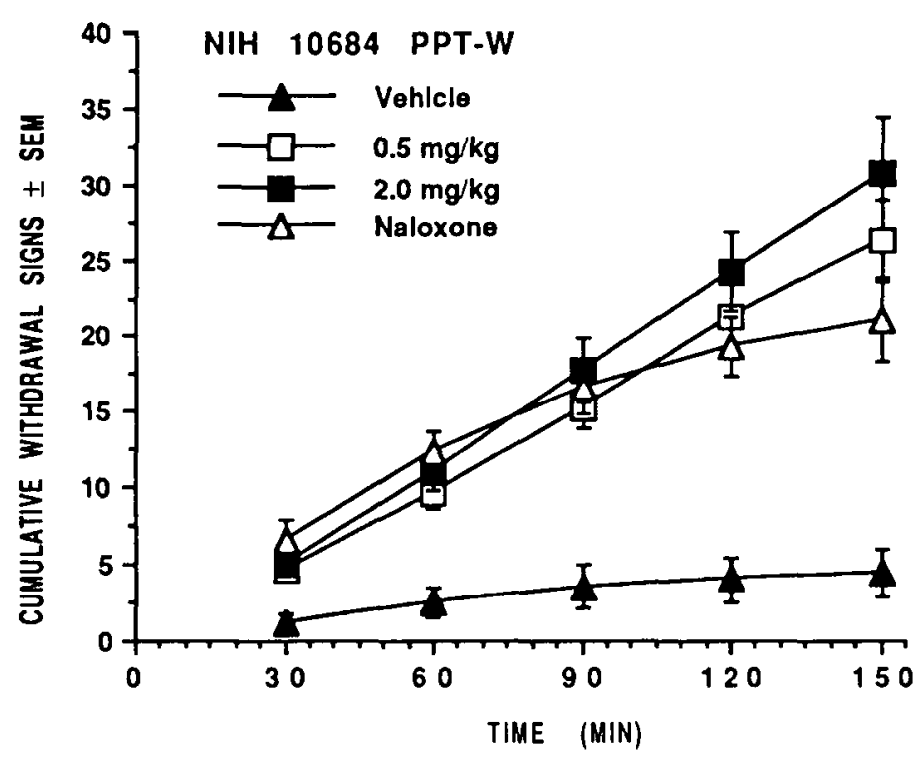

\section{Comments:}

NM 10684 has mixed agonist/antagonist properties in the mouse. In the monkey, the drug has mu antagonist properties. Onset of action is slow and offset is of long duration. This compound appears to be either devoid of mu antinociceptive activity or lacks potency. 


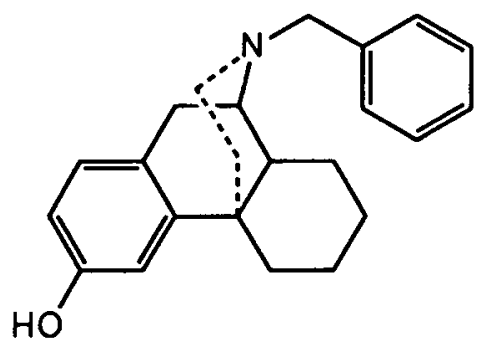

MOUSE DATA - ED50 OR AD50, $\mathrm{mg} / \mathrm{kg}$

(95\% C.L.) or \% change

1) $\mathrm{TF}$ - Inactive at 1.0, 10.0 and $30.0^{\mathrm{a}}$

2) $\mathrm{TF}$ vs. $\mathrm{M}$ - Inactive at $1.0,10.0$ and $30.0^{\mathrm{a}}$

3) PPQ - 6.1(2.0 - 19.2) $)^{\mathrm{a}}$

4) $\mathrm{HP}$ - Inactive at 1.0 and 10.0, $25 \%$ at $30.0^{\mathrm{a}, \mathrm{b}}$

${ }^{\mathrm{a}}$ Vehicle - DMSO, propylene glycol and water

${ }^{\mathrm{b}}$ Some eyelid ptosis, convulsions in one mouse at 30.0

\section{MONKEY DATA}

(SDS)

In the preliminary study, shortly after receiving a cumulative dose of $15.0 \mathrm{mg} / \mathrm{kg}$ $(1.0,2.0,4.0$ and $8.0 \mathrm{mg} / \mathrm{kg}$ at $15-\mathrm{m}$ intervals $)$, the monkey dropped from the perch and convulsed. Pentobarbital quickly terminated the convulsions.

In the regular SDS study, at doses of 1.75 and $7.0 \mathrm{mg} / \mathrm{kg}$, NIH $10735 \mathrm{did}$ not substitute for morphine or exacerbate withdrawal (see fig.). Vehicle was $25 \%$ hydroxypropyl- $\beta$-cyclodextrin.

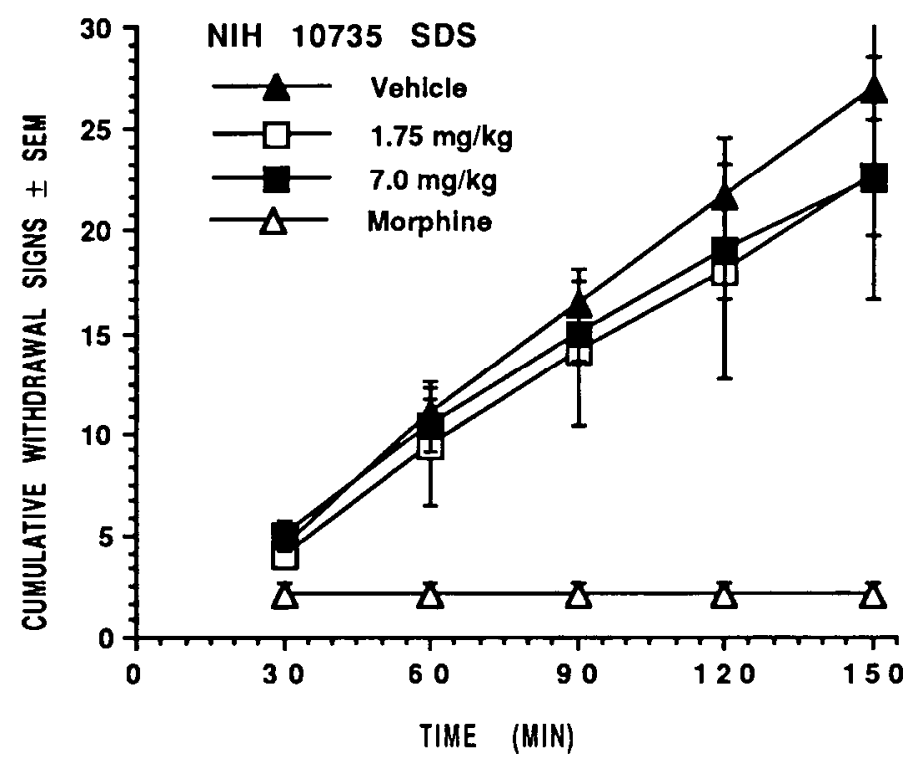


NIH 10738 4-(3-Hydroxyphenyl)-1-(4-nitrobenzyl)-1-4-(1-oxopropyl) piperidine hydrochloride

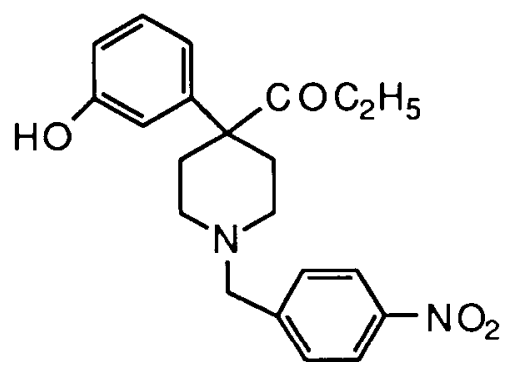

MOUSE DATA - ED50 OR AD50, $\mathrm{mg} / \mathrm{kg}$

(95\% C.L.) or \% change

1) $\mathrm{TF}$ - Inactive at $1.0,10.0$ and $30.0^{\mathrm{a}}$

2) $\mathrm{TF}$ vs. $\mathrm{M}$ - Inactive at $1.0,10.0$ and $30.0^{\mathrm{a}}$

PPQ - $7.3(2.2-24.7)^{\mathrm{a}}$

$\mathrm{HP}$ - Inactive at $1.0,15 \%$ at

10.0 and $30.0^{\mathrm{a}}$

Vehicle - DMSO in water

\section{MONKEY DATA}

(SDS)

As shown in the accompanying fig. (NIH 10738, SDS), this compound neither substituted for morphine nor exacerbated withdrawal at doses of 3.0 or 12.0 $\mathrm{mg} / \mathrm{kg}$. Vehicle was $25 \%$ hydroxypropyl- $\beta$-cyclodextrin in water.

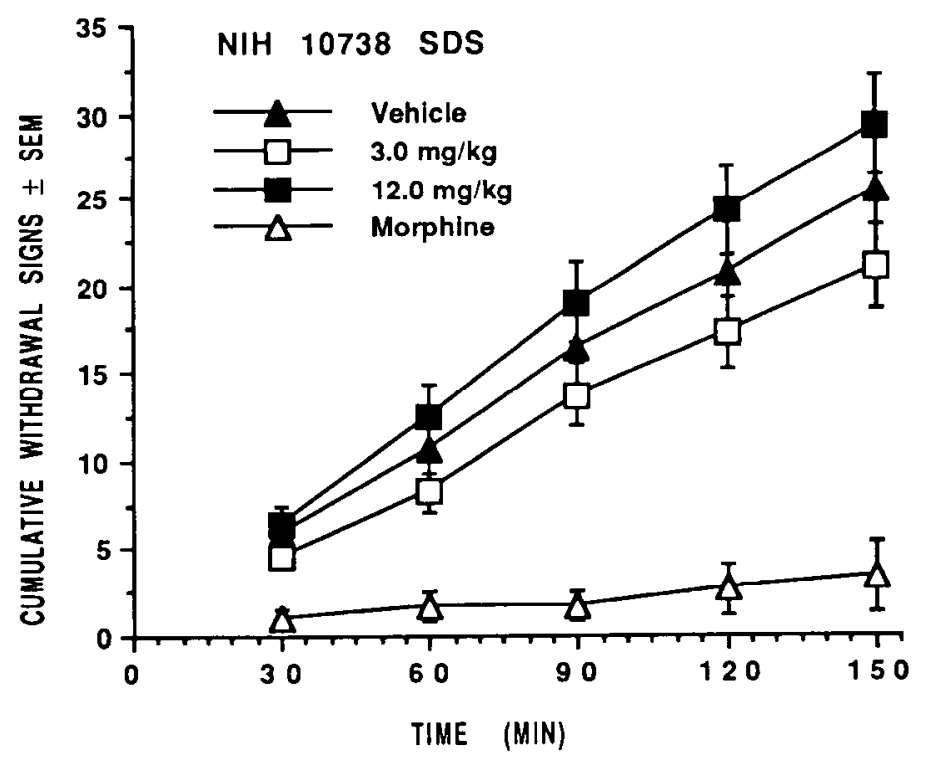

\section{Comments:}

Although some antinociceptive activity was noted, the results do not suggest opioid properties. 
NIH 10739 1-(4-Fluorobenzyl)-4-(3-hydroxyphenyl)-4-(1-oxopropyl)piperidine hydrochloride

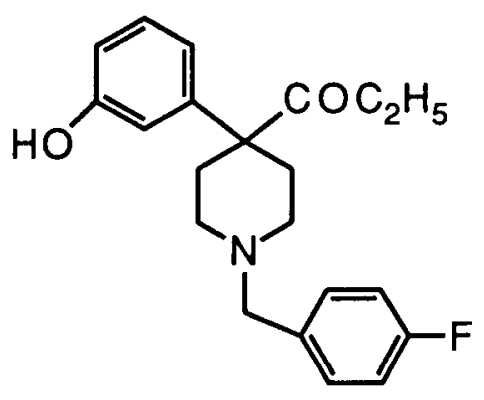

MOUSE DATA - ED50 OR AD50, $\mathrm{mg} / \mathrm{kg}$

(95\% C.L.) or \% change

1) $\mathrm{TF}$ - Inactive at $1.0,10.0$ and $30.0^{\mathrm{a}}$

2) $\mathrm{TF}$ vs. $\mathrm{M}$ - Inactive at $1.0,10.0$ and $30.0^{\mathrm{a}}$

3) PPQ - $18.3(9.7-34.7)^{\mathrm{a}}$

4) $\mathrm{HP}$-Inactive at 1.0 and 10.0 , $25 \%$ at $30.0^{\mathrm{a}}$

${ }^{\mathrm{a}}$ Vehicle - DMSO in water.

\section{MONKEY DATA}

(SDS)

At doses of 2.5 and $10.0 \mathrm{mg} / \mathrm{kg}, \mathrm{NIH} 10739$ did not show appreciable activity in morphine-dependent monkeys, i.e., it neither substituted for morphine nor exacerbated withdrawal. Vehicle was $25 \%$ hydropropyl $\beta$-cyclodextrin aqueous solution.

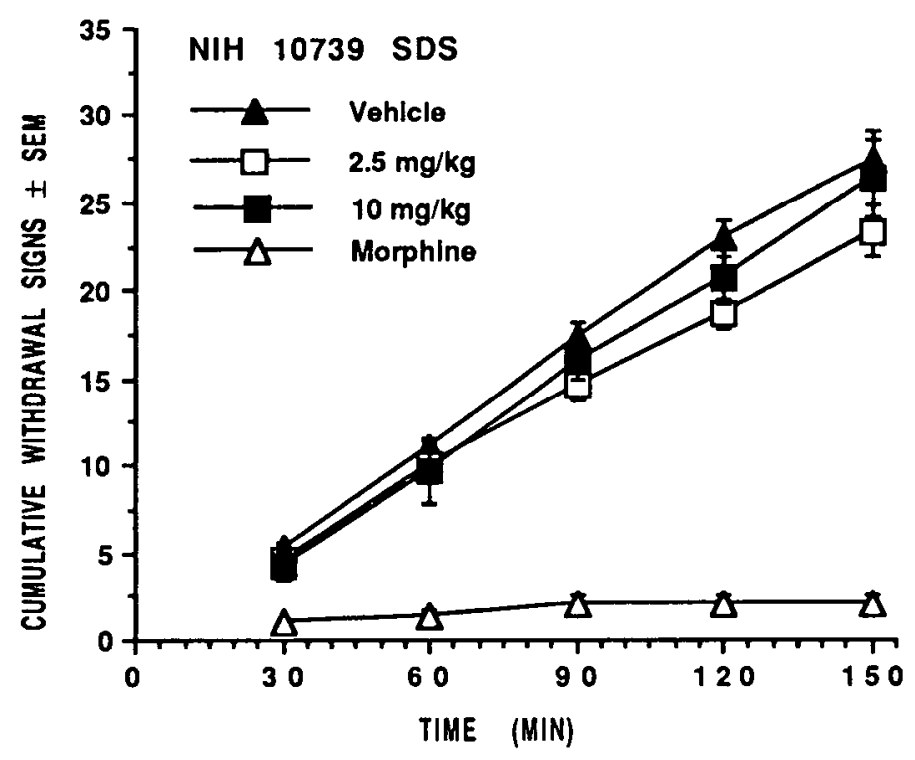

\section{Comments:}

The drug has weak antinociceptive activity. The biological profile suggests that it lacks significant opioid activity. 
NIH 10741 ( $\beta$ S,3R,4S)-(+)-cis-N-[1-(2( $\beta$ )-Hydroxy-2-phenylethyl)-3methyl-4-piperidinyl]-N-phenylpropanamide hydrochloride

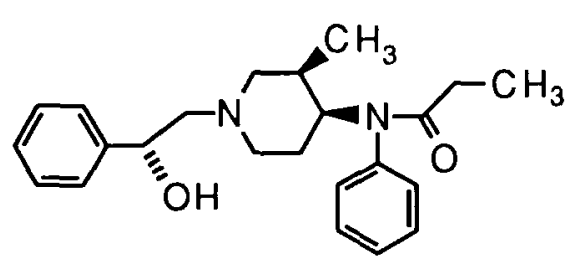

MOUSE DATA-ED50 OR AD50 (95\% C.L.) or \% change $(\mathrm{mg} / \mathrm{kg})$

1) $\mathrm{TF}-2 \times 10^{-4}\left(1 \times 10^{-4}-3 \mathrm{x}\right.$ $\left.10^{-4}\right)^{\mathrm{a}}$

2) TF vs. M - Inactive at 1.0, 10.0 and $30.0^{\mathrm{a}}$

3) PPQ - $9 \times 10^{-5}\left(3 \times 10^{-5}-2 \times\right.$ $\left.10^{-4}\right)^{\mathrm{a}}$

4) $\mathrm{HP}-1 \times 10^{-4}\left(5 \times 10^{-5}-2 \times\right.$ $\left.10^{-4}\right)^{\mathrm{a}}$

${ }^{a}$ Reported Previously. NIDA Res. Monog. (in press).

\section{MOUSE DATA}

Special Test: (op cit) Naloxone vs ED80 of NIH 10741 in TF: AD50 $=8.3 \mathrm{x}$ $10^{-3}\left(4 \times 10^{-3}-1.7 \times 10^{-2}\right)$

$\mathrm{pA}_{2}$ Assay NIH 10741: $\quad \mathrm{A} \mathrm{pA}_{2}$ of 7.2 and slope of -0.98 was calculated. The results suggest a competitive interaction with the mu receptor (see accompanying fig.).

NALOXONE VERSUS NIH 10741 IN TAILFLICK Apparent pA2 Schild

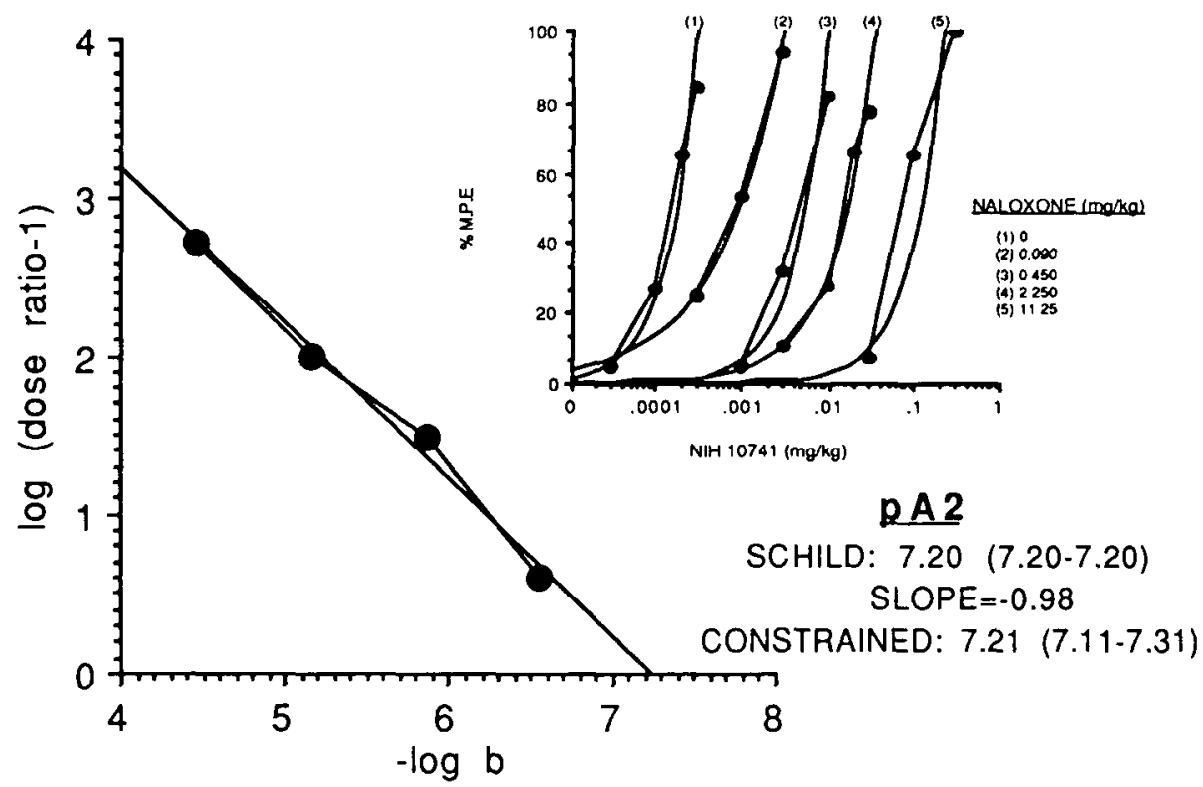


NIH $10741 \quad \beta$ :S,3R,4S)-(+)- c is -N-[1-(2( $\boldsymbol{\beta})$-Hydroxy-2-phenylethyl)-3methyl-4-piperidinyl]-N-phenylpropanamide hydrochloride (continued)

\section{MONKEY DATA \\ SDS}

Reported previously - op cit. Substituted completely for morphine 20,000 $50,000 \times$ more potent than morphine.

NIH 10741 (ßS,3R,4S)-(+)-cis-N-[1-(2(ß)-Hydroxy-2-phenylethyl)-3methyl-4-piperidinyl]-N-phenylpropanamide hydrochloride (continued)

\section{Comments:}

Because this compound was extremely potent and because the naloxone AD50 suggested unusual sensitivity to naloxone, the pA2 test was conducted. The results indicate that $\mathrm{NIH} 10741$ interacts competively with naloxone at the mu receptor.

NIH 10749 (+)-3-Hydroxy-N-(4-nitrobenzyl)morphinan oxalate

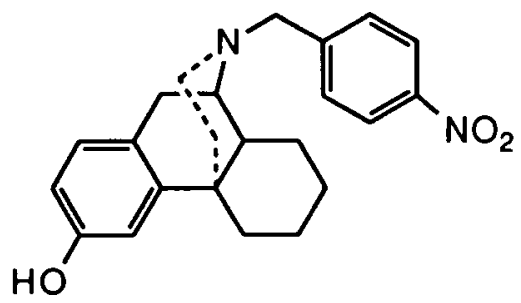

MOUSE DATA - ED50 OR AD50, $\mathrm{mg} / \mathrm{kg}$

(95\% C.L.) or $\%$ change

1) $\mathrm{TF}$ - Inactive at 1.0, 10.0 and $30.0^{\mathrm{a}}$

2) $\mathrm{TF}$ vs. $\mathrm{M}$ - Inactive at $1.0,10.0$ and $30.0^{\mathrm{a}}$

3) PPQ - $23 \%$ at $1.0,14 \%$ at 10.0 and $54 \%$ at $30.0^{\mathrm{a}}$

4) HP - Inactive at 1.0, 10.0 and $30.0^{\mathrm{a}}$

${ }^{\mathrm{a}}$ Vehicle - Tween 80,

Propyleneglycol and water

\section{MONKEY DATA}

SDS

As shown in the graph (NIH 10749 SDS), at doses of 4.0 or $16.0 \mathrm{mg} / \mathrm{kg}$, this compound did not substitute for morphine. Neither did it exacerbate withdrawal. Vehicle was $25 \%$ hydroxypropyl- $\beta$-cyclodextrin. 


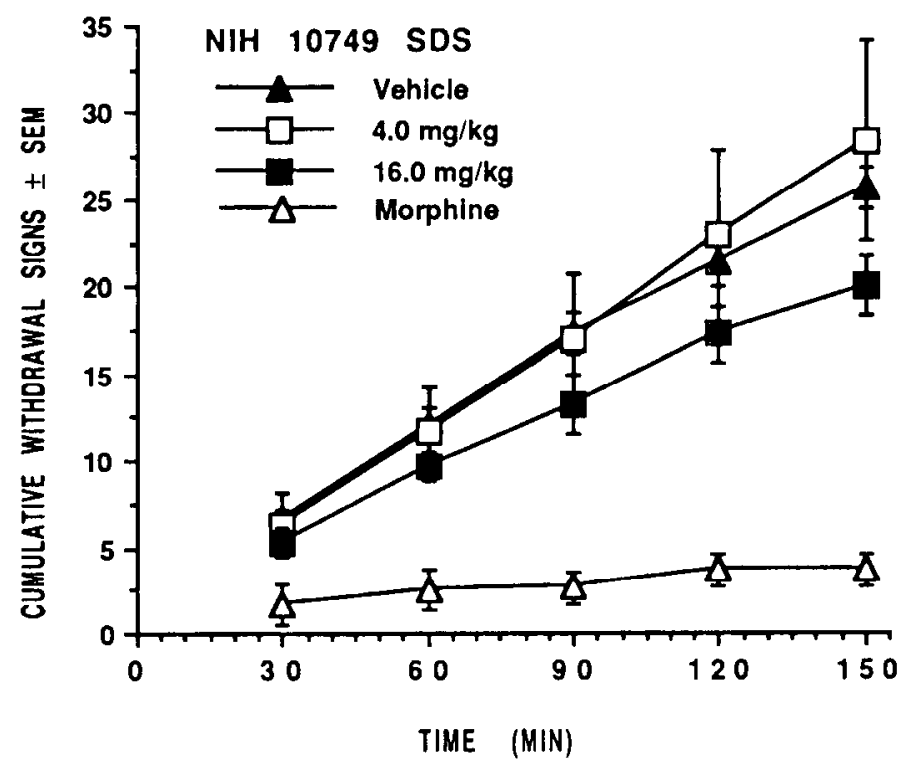

\section{Comments:}

This compound did not display remarkable activity in the mouse antinociceptive tests and in morphine-dependent monkeys, It is probably devoid of mu and kappa opioid properties.

NIH 10773 Morphine 3-acetate 6-sulfate zwitterion

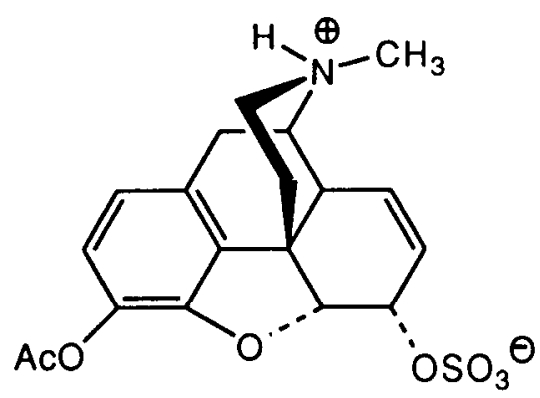

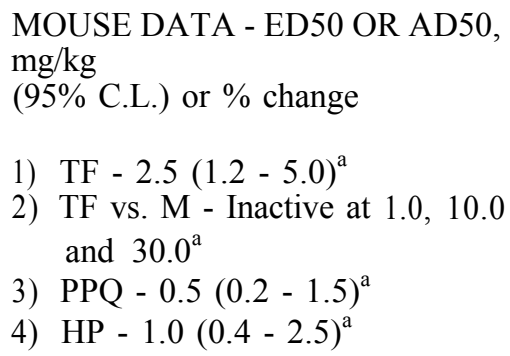

MOUSE DATA - ED50 OR AD50, $\mathrm{mg} / \mathrm{kg}$

(95\% C.L.) or \% change

1) $\mathrm{TF}-2.5(1.2-5.0)^{\mathrm{a}}$

2) $\mathrm{TF}$ vs. $\mathrm{M}$ - Inactive at $1.0,10.0$ and $30.0^{\mathrm{a}}$

3) PPQ - $0.5(0.2-1.5)^{\mathrm{a}}$

4) $\mathrm{HP}-1.0(0.4-2.5)^{\mathrm{a}}$

${ }^{\mathrm{a}}$ Vehicle - 25\% Tween 80, water with gentle warming 
NIH 10773 Morphine 3-acetate 6-sulfate zwitterion (continued)

$\frac{\text { MONKEY DATA }}{\text { SDS }}$

At doses of 2.5 and $10.0 \mathrm{mg} / \mathrm{kg}$, NIH 10773 did not substitute for morphine nor did it exacerbate withdrawal. Vehicle was DMSO $10-15 \%$ in water. Solutions were cloudy.

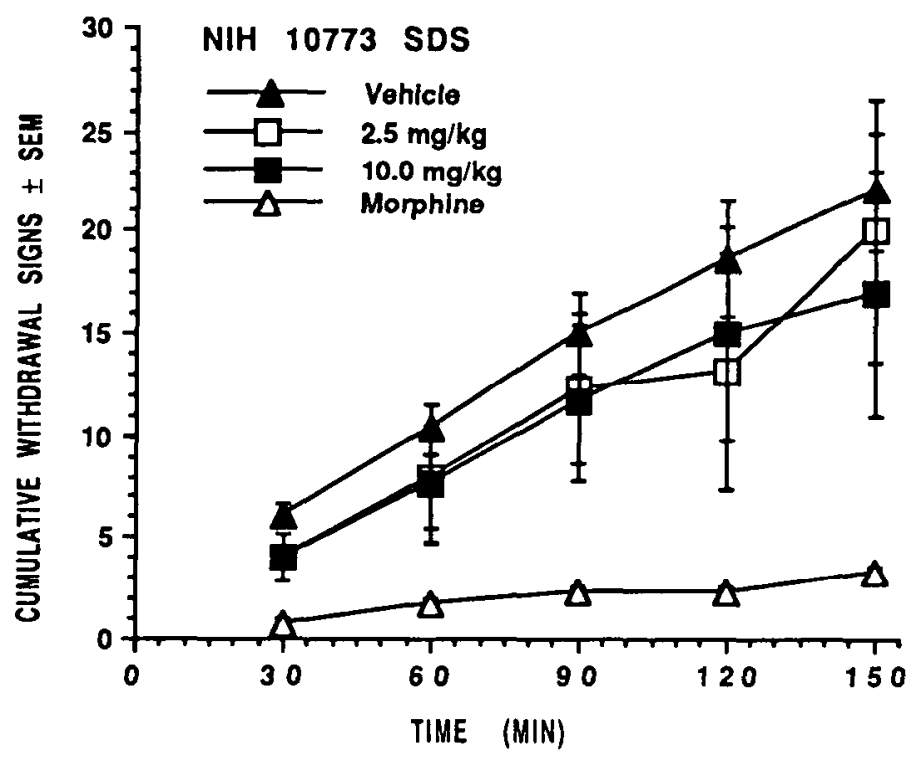

Comments:

The results suggest species difference and/or solubility problems. In any case, in the mouse antinociceptive models, NIH 10773 appears to be morphine-like.

NM 10779 2,3-Dimethyl-5-(3-hydroxyphenyl)morphan hydrochloride

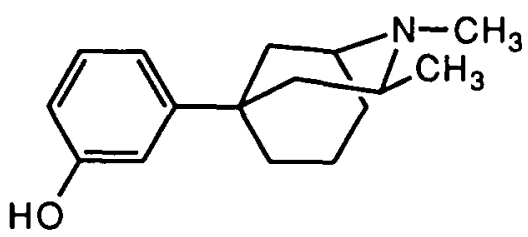
MOUSE DATA - ED50 OR $\mathrm{AD} 50, \mathrm{mg} / \mathrm{kg}$ (95\% C.L.) or \% change

1) $\mathrm{TF}-1.3(0.5-3.2)$

2) $\mathrm{TF}$ vs. $\mathrm{M}$ - Inactive at 1.0 , 10.0 and 30.0

3) PPQ - $0.3(0.1-0.6)$

4) $\mathrm{HP}-4.8(1.9-12.4)$ 
NIH 10779 2,3-Dimethyl-5-(3-hydroxyphenyl)morphan hydrochloride (continued)

\section{MONKEY DATA \\ SDS}

NIH 10779 substituted completely for morphine (see fig. NIH 10779 SDS).

Potency, onset of action and duration of action appear comparable to morphine.

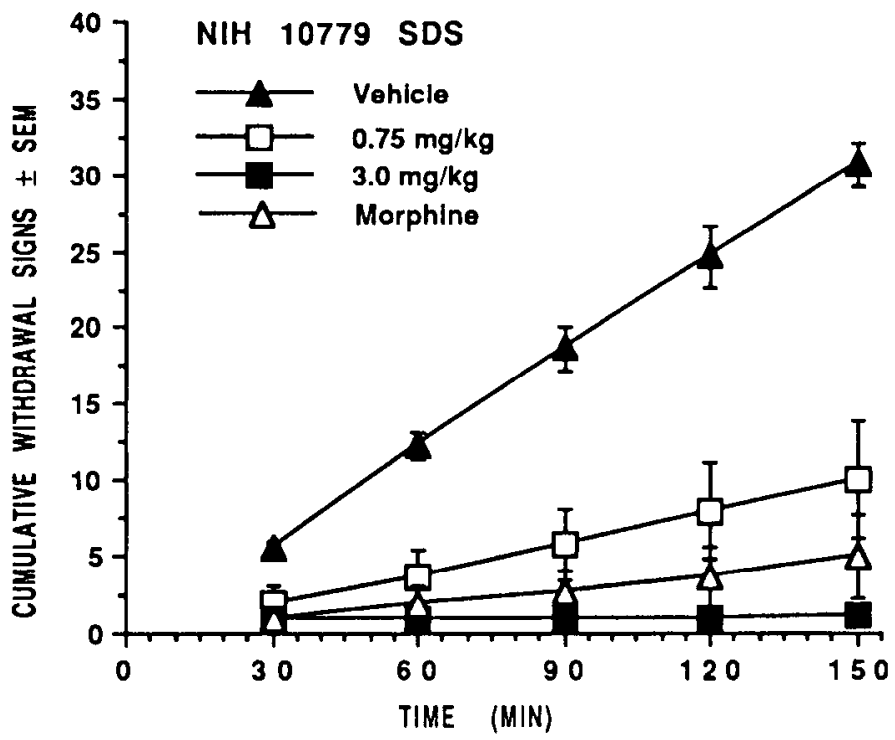

Comments:

This drug displays a profile of activity similar to that of morphine. 


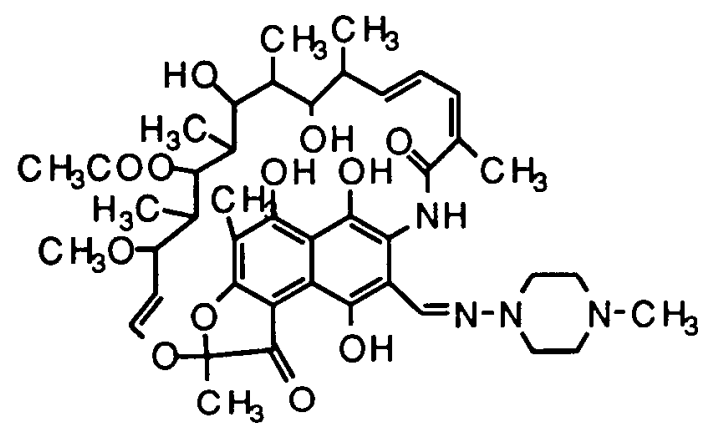

MOUSE DATA - ED50 OF AD $50, \mathrm{mg} / \mathrm{kg}$ (95\% C.L.) or \% change

1) $\mathrm{TF}$ - Inactive at 1.0, 10.0 and $30.0^{\mathrm{a}}$

2) $\mathrm{TF}$ vs. M - Inactive at $1.0,10.0$ and $30.0^{\mathrm{a}}$

3) PPQ - Inactive at 1.0, 10.0 and $30.0^{\mathrm{a}}$

4) $\mathrm{HP}$ - Inactive at $1.0,10.0$ and $30.0^{\mathrm{a}}$

${ }^{\mathrm{a}}$ Vehicle $0.5 \%$ Tween 80 in aqueous solution.

\section{MONKEY DATA}

Preliminary (SDS)

$\mathrm{NIH} 10782$ was injected at $15-\mathrm{m}$ intervals with doses of 1.0, 2.0, 4.0 and 4.0 $\mathrm{mg} / \mathrm{kg}$, respectively for a total cumulative dose of $11.0 \mathrm{mg} / \mathrm{kg}$. Withdrawal signs were neither attenuated nor suppressed. Because the drug formed a reddish solution which stained tissue, there was concern regarding the production of skin lesions in monkeys, no further studies were initiated. Vehicle was hydroxypropyl $\beta$-cyclodextrin in water.

\section{Comments:}

The results from this preliminary study suggested that the compound had no apparent mu or kappa opioid activity.

NIH $10784 \quad( \pm)-c i s-\mathrm{N}-[1-(2-H y d r o x y-2-p h e n y l e t h y l)-3-m e t h y l-4-$ piperidinyl]-N-(3-fluorophenyl)propanamide hydrochloride

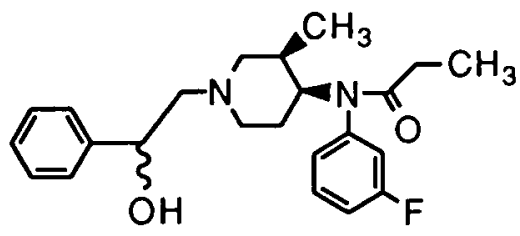

MOUSE DATA - ED50 OR AD50, $\mathrm{mg} / \mathrm{kg}$

( $95 \%$ C.L.) or $\%$ change

1) $\mathrm{TF}-0.004(0.001-0.009)$

2) $\mathrm{TF}$ vs. $\mathrm{M}$ - Inactive at $1.0,10.0$ and $30.0^{\mathrm{a}}$

3) PPQ $-0.001(0.005-0.003)$

4) $\mathrm{HP}-0.005(0.002-0.01)$

${ }^{\mathrm{a}}$ Straub tail at 1.0. Loss of righting reflex at 30.0 and 10.0 
NIH 10784 ( \pm )-cis-N-[1-(2-Hydroxy-2-phenylethyl)-3-methyl-4piperidinyl]-N-(3-fluorophenyl)propanamide hydrochloride (continued)

Special test: Naloxone vs ED80 of NM 10784 in TF - AD50 $=0.11(0.04$ 0.33)

\section{MONKEY DATA}

(SDS)

NIH 10784 is a highly potent (6000 x morphine) mu opioid agonist and, accordingly, substituted completely for morphine in morphine-dependent monkeys. Onset of action was rapid and duration of action was at least as long as that of morphine. See accompanying fig.

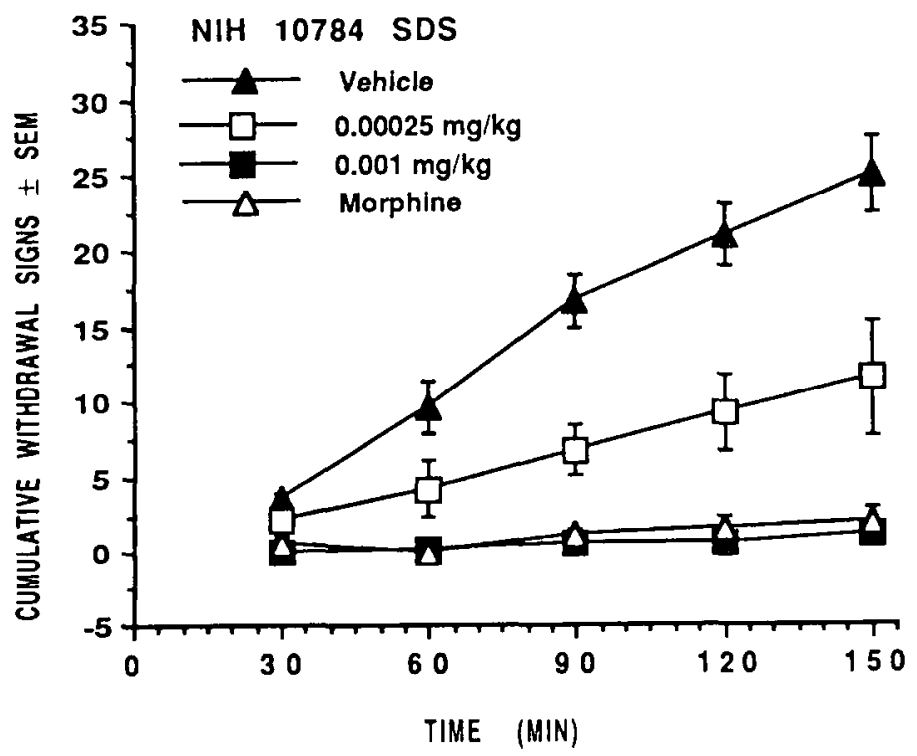

Comments:

This compound is a potent mu agonist. 
NIH $10785 \quad( \pm)$-c is -N-[1-(2-Hydroxy-2-phenylethyl)-3-methyl-4piperidinyl]-N-(4-fluorophenyl)propanamide hydrochloride

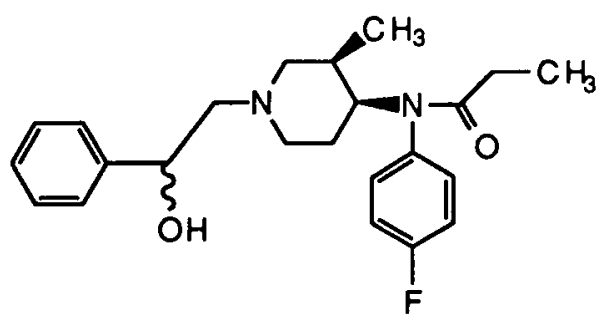

MOUSE DATA - ED50 OF AD $50, \mathrm{mg} / \mathrm{kg}$ (95\% C.L.) or \% change

1) $\mathrm{TF}-0.002(0.001-0.005)$

2) $\mathrm{TF}$ vs. $\mathrm{M}$ - Inactive at 1.0 , 30.0 and $10.0^{\mathrm{a}}$

3) PPQ - $0.00034(0.00012$ 0.00093 )

4) $\mathrm{HP}-0.001(0.0004$ $0.0029)$

at $1.0 \mathrm{mg} / \mathrm{kg}$ dose of righting reflex

Special Test: Naloxone AD50 vs ED80 of NM 10785 in TF $=0.04(0.02-0.09)$

\section{MONKEY DATA}

(SDS)

As can be seen in the figure, NM 10785 produced a dose-related and complete suppression of withdrawal signs. Onset of action was rapid, offset was morphine-like. Potency estimate is $1000 \mathrm{x}$ morphine. Some scratching was noted at the high dose.

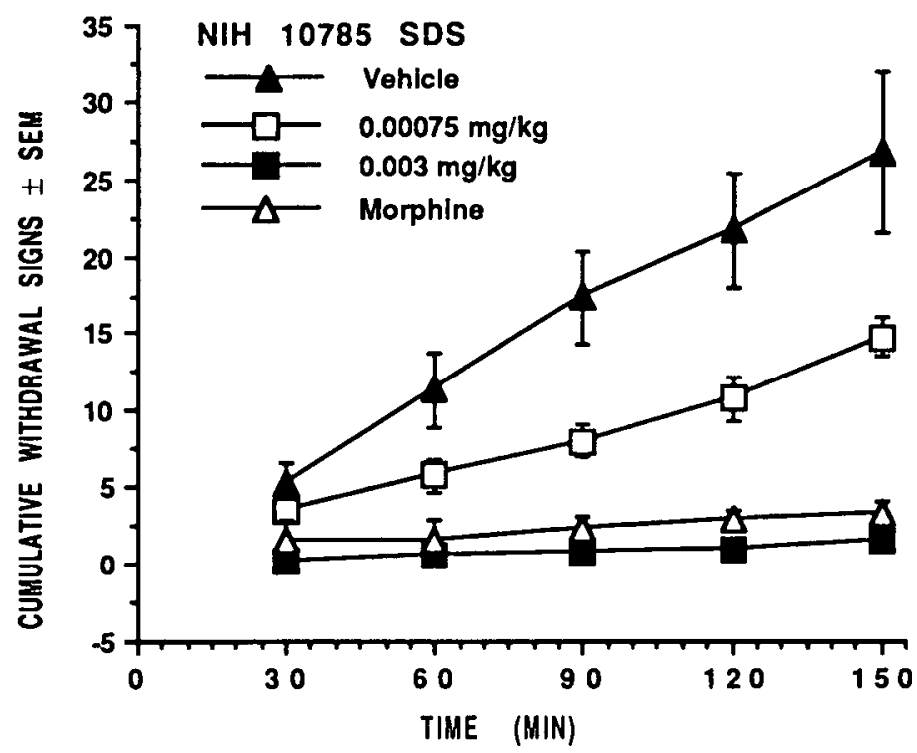

Comments:

The profile of activity indicates that NM 10785 is a potent mu agonist. 
NIH 10786 ( \pm )-cis-N-[1-[1-(4-Bromophenyl)ethyl-2-hydroxyl-3-methyl-4piperidyl]-N-phenylpropanamide $\bullet \mathrm{HCl}$

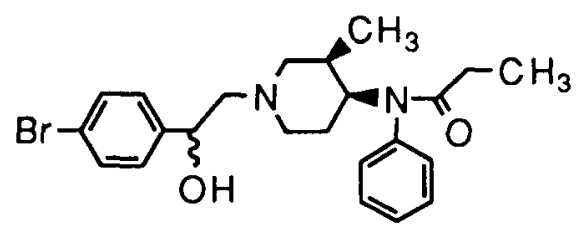

MOUSE DATA - ED50 OR AD50, $\mathrm{mg} / \mathrm{kg}$

( $95 \%$ C.L.) or $\%$ change

1) $\mathrm{TF}-0.03(0.22-0.51)$

2) $\mathrm{TF}$ vs. M - Inactive at 1.0, 10.0 and 30.0

3) PPQ - $0.007(0.003-0.018)$

4) $\mathrm{HP}-0.013(0.006-0.032)$

Special: Naloxone AD50 vs ED80 of 10786 in TF - 0.13 (0.05 - 0.32)

\section{MONKEY DATA}

A. ( SDS)

NIH 10786 dose-dependently substituted completely for morphine (see graph). This compound is estimated to be $60 \mathrm{x}$ more potent than the reference standard morphine sulfate. The drug acts quickly and its duration of action is at least as long as that of morphine.

\section{Comments:}

The profile of activity suggests that NIH 10786 has potent mu agonist properties.

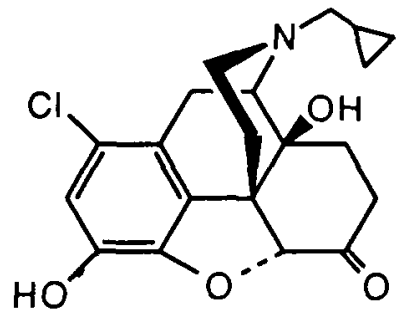

MOUSE DATA - ED50 OR AD50, $\mathrm{mg} / \mathrm{kg}$

$(95 \%$ C.L.) or $\%$ change

1) $\mathrm{TF}$ - Inactive at $1.0,10.0$ and 30.0

2) $\mathrm{TF}$ vs. $\mathrm{M}-4.8 \times 10^{-4}\left(1.9 \times 10^{-4}\right.$ $\left.\mathrm{x} 1.2 \times 10^{-3}\right)$

3) PPQ - Inactive at 1.0, 10.0 and 30.0

4) $\mathrm{HP}$ - Inactive at 1.0, 10.0 and 30.0

\section{MOUSE DATA}

1. $\mathrm{pA}_{2}$ determination vs morphine in $\mathrm{TF}$ test

As shown in the accompanying fig., NIH 10787 competitively antagonized morphine-induced antinociception. The $\mathrm{pA}_{2}$ was calculated at 8.7 which indicates that the affinity for the mu receptor is much greater than that of naloxone or nalmefene. 
$\mathrm{NIH} 10787$ 1-Chloronaltrexone $\cdot \mathrm{HCl}$ (continued)

NIH 10787 VERSUS MORPHINE IN TAIL-FLICK Apparent pA2 Schild

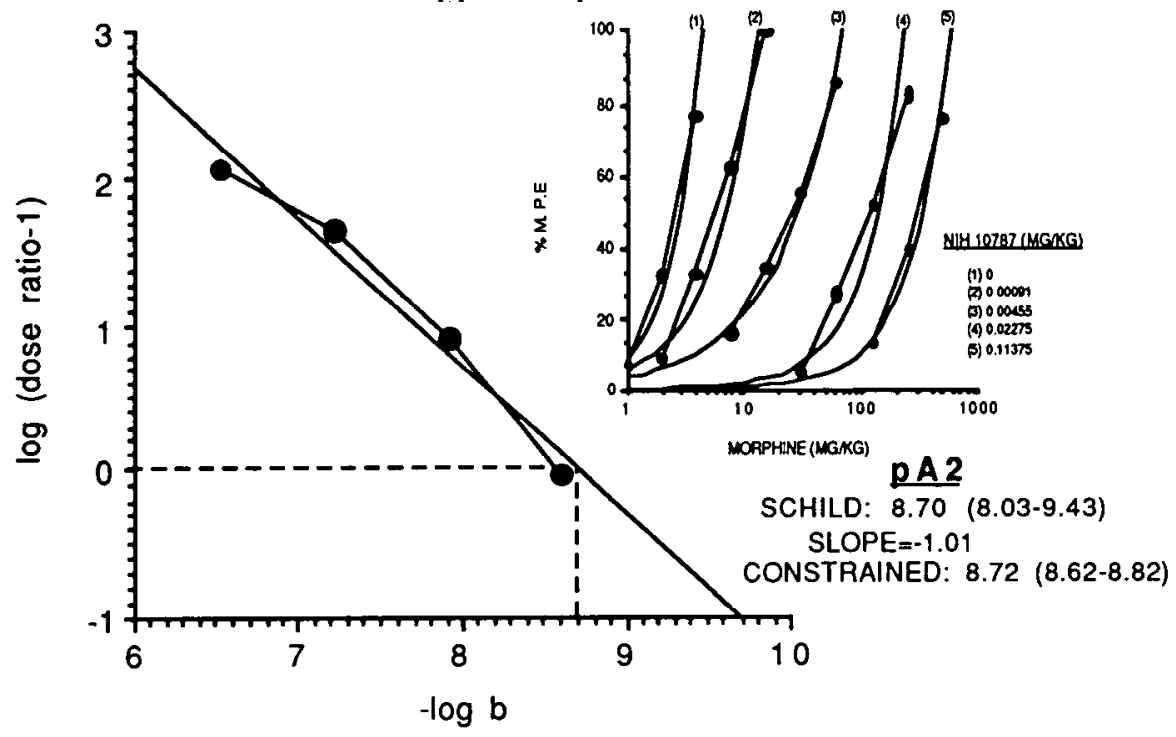

2. $\underline{\mathrm{pA}}_{2}$ determination vs NIH 10672 (Kappa agonist) in TF test

NIH 10787 competitively antagonized antinociception induced by the potent kappa agonist NIH 10672. The affinity of 10787 for the kappa receptor is also impressive.

NIH 10787 VERSUS NIH 10672 IN TAIL-FLICK Apparent pA2 Schild

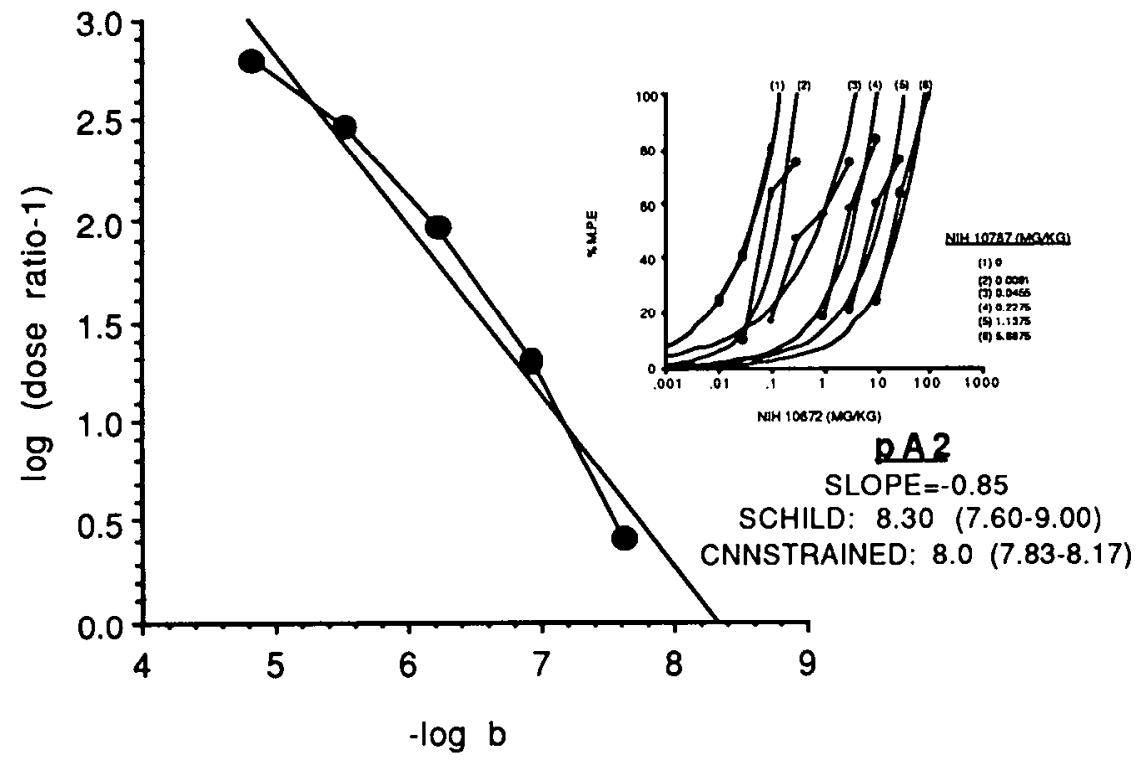


NIH 10787 1-Chloronaltrexone $\cdot \mathrm{HCl}$ (continued)

MONKEY DATA

A. (SDS)

As illustrated in the fig., NIH 10787 SDS, NIH 10787 did not substitute for morphine at doses of 0.0004 or $0.0016 \mathrm{mg} / \mathrm{kg}$.

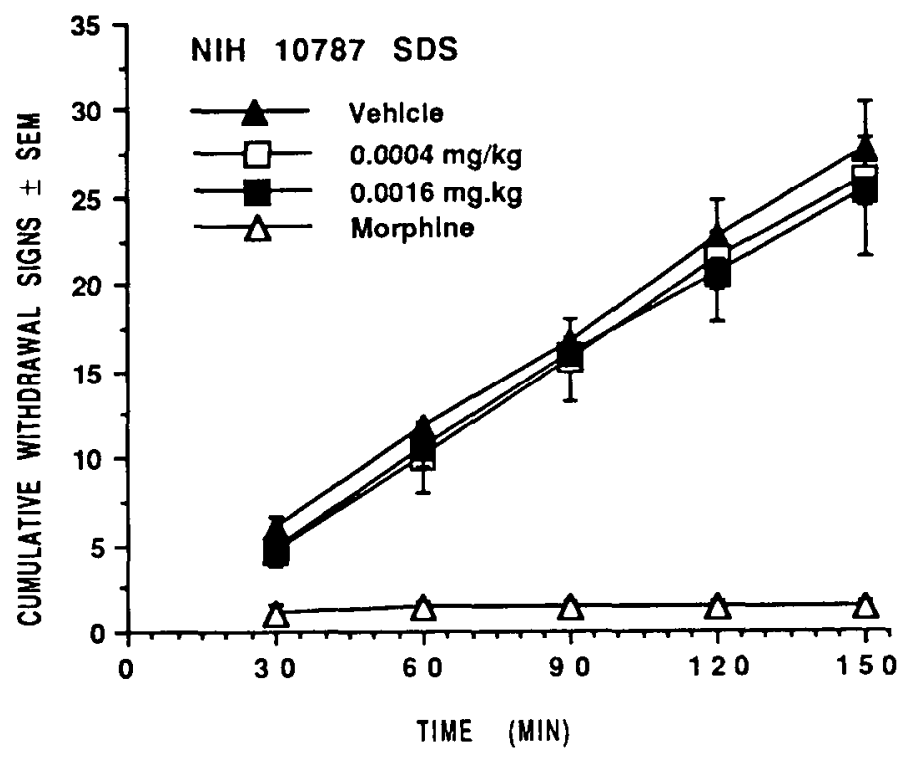




\section{B. (PPt-W)}

NIH 10787 dose-dependently precipitated withdrawal in morphine-dependent monkeys. Onset of action was prompt and duration of action was at least as long as that of naloxone. Potency estimate in the monkey is approximately $5 \mathrm{x}$ that of naloxone.

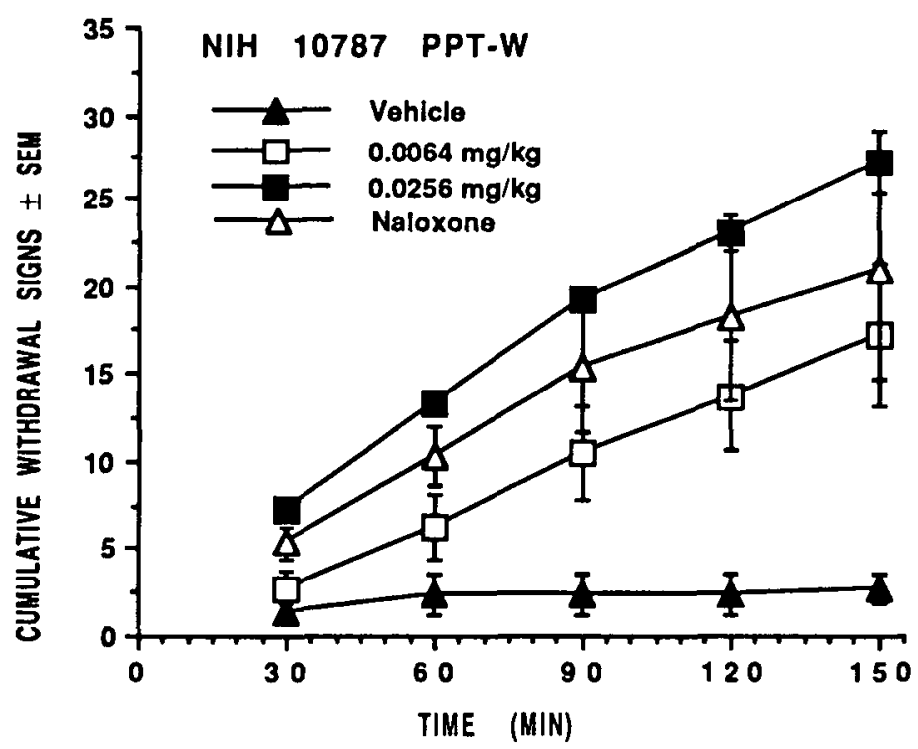

\section{Comments;}

In the mouse, NIH 10787 is an extraordinarily potent competitive antagonist especially at mu and to a lessor extent on kappa receptors. Although the drug is active in the monkey model, potency is only approximately $5 \mathrm{x}$ that of naloxone. Perhaps the species differences can be reconciled by metabolic studies. 
NIH $10790 \quad( \pm)-1-(2-H y d r o x y-2-p h e n y l e t h y l)-t-3-m e t h y l-4-[(1-$ oxopropyl)phenylamino]-r-4-piperidinecarboxamide methyl ester $\bullet \mathrm{HC} 1$

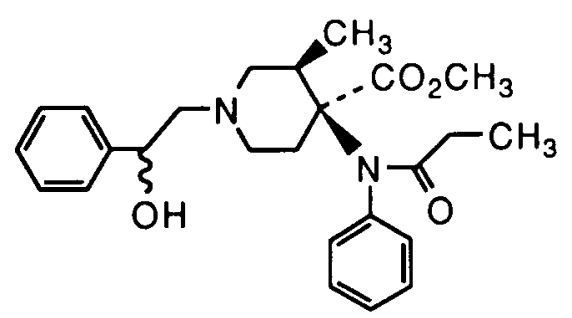

MOUSE DATA - ED50 OR AD50, $\mathrm{mg} / \mathrm{kg}$

(95\% C.L.) or \% change

1) $\mathrm{TF}-4.4 \times 10^{-4}\left(2.2 \times 10^{-4}-\right.$ $\left.8.7 \times 10^{-4}\right)^{\mathrm{a}}$

2) $\mathrm{TF}$ vs. $\mathrm{M}$ - Inactive at $0.1,0.3$ and $1.0^{\mathrm{b}}$

3) PPQ - $1.0 \times 1.0^{-4}$

4) $\mathrm{HP}-6.1 \times 10^{-4}\left(2.8 \times 10^{-4}\right.$ $\left.1.3 \times 10^{-3}\right)^{\mathrm{a}}$

${ }^{\mathrm{a}}$ Straub tail at $0.001 \mathrm{mg} / \mathrm{kg}$

${ }^{\mathrm{b}}$ Two of 6 mice died at 1.0

Special Test: A: Naloxone AD50 vs ED50 of NIH 10790 in $\mathrm{TF}=0.02$ $(0.006-0.09)$

B: $\quad \mathrm{pA}_{2}$ (Naloxone vs NIH 10790) 7.4 (Schild); 7.1 (constrained). See accompanying illustrated data.

NALOXONE VERSUS NIH 10790 IN TAIL-FLICK Apparant pA2 (Schild)

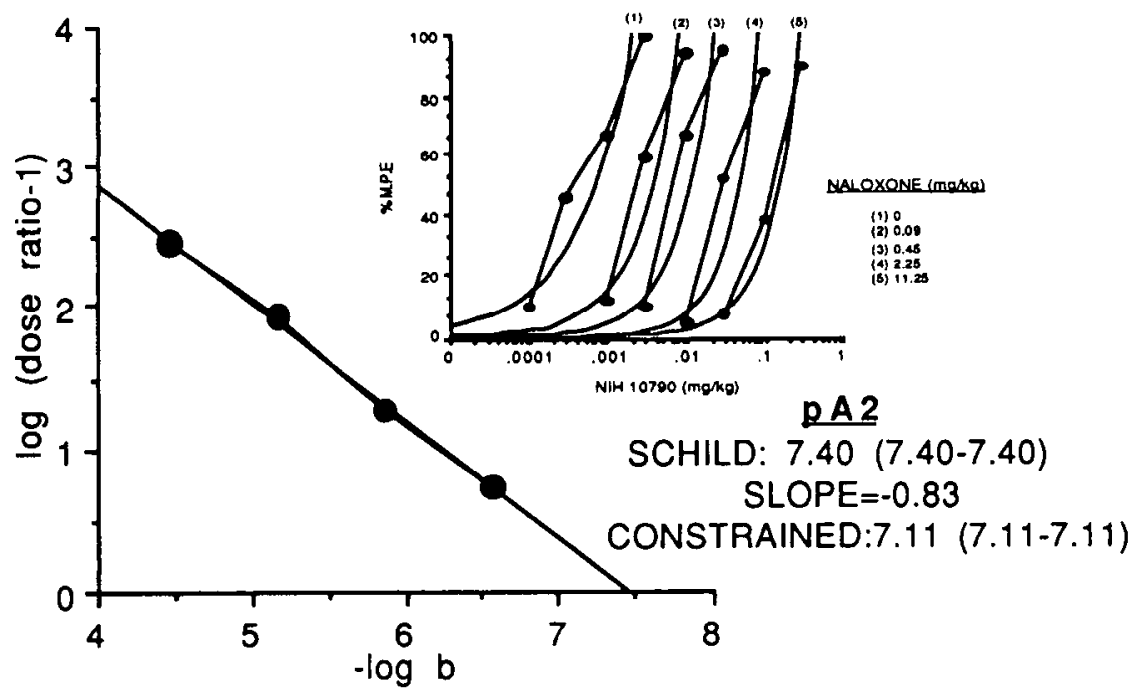


NIH 10790 ( \pm )-1-(2-Hydroxy-2-phenylethyl)-t-3-methyl-4-[(1oxopropyl)phenylamino]-r-4-piperidinecarboxamide methyl ester $\bullet \mathrm{HC} 1$ (continued)

\section{MONKEY DATA}

(SDS)

This compound dose-dependently substituted completely for morphine (see fig.). Onset and offset of action are similar to those of morphine. Potency estimate: $6000 \mathrm{x}$ morphine.

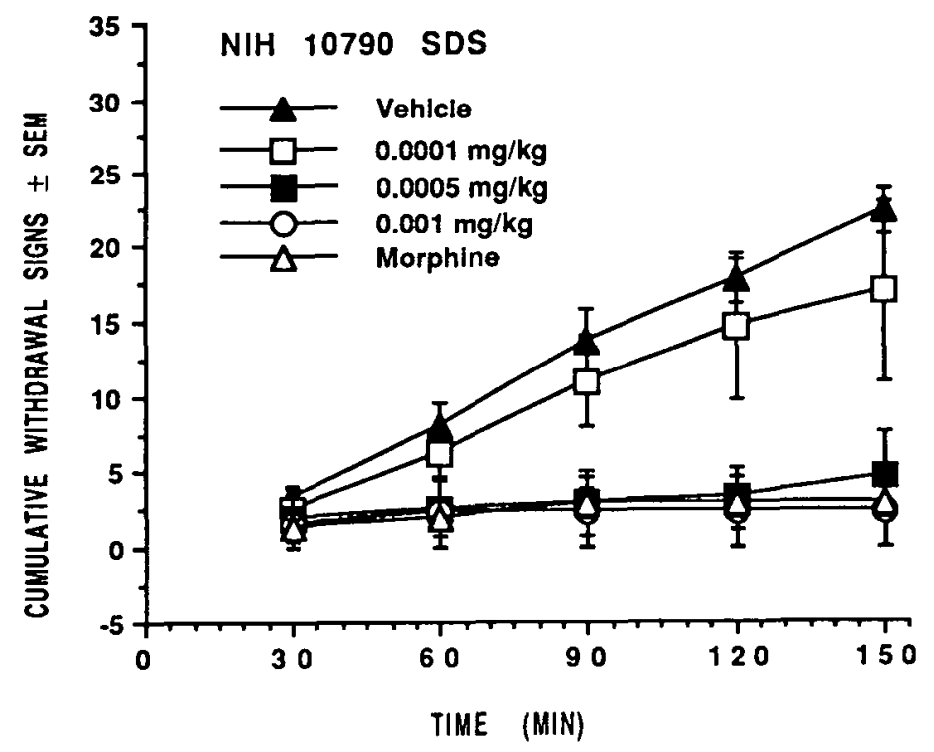

Comments:

All the data indicate that $\mathrm{NIH} 10790$ is a potent mu agonist that interacts competitively with naloxone. 
NIH 10791 ( \pm )-1-(2-Hydroxy-2-phenylethyl)-c-3-methyl-4-[(1oxopropyl)phenylamino]-r-4-piperidinecarboxylic acid methyl ester hydrochloride

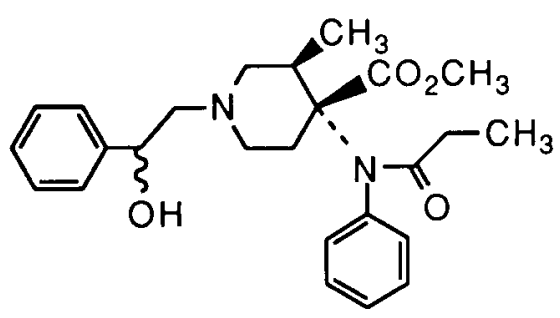

MOUSE DATA - ED50 OR AD50, $\mathrm{mg} / \mathrm{kg}$

(95\% C.L) or \% change

1) $\mathrm{TF}-0.001(0.0004-0.003)^{\mathrm{a}}$

2) $\mathrm{TF}$ vs $\mathrm{M}$ - Inactive at $1.0,10.0$ and $30.0^{\mathrm{b}}$

3) PPQ - $8.0 \times 10^{-4}\left(3.0 \times 1.0^{-4} \times 2.1\right.$ $\left.\mathrm{X} 10^{-4}\right)$

4) $\mathrm{HP}-0.001(0.0005-0.002)^{\mathrm{a}}$

${ }^{\mathrm{a}}$ Straub tail at $0.003 \mathrm{mg} / \mathrm{kg}$

${ }^{\mathrm{b}}$ One mouse died at 30.0

Special Test: A: Naloxone AD50 vs ED80 of NIH 10791 in TF $=0.02(0.01$ $0.04)$

B: $\mathrm{pA}_{2}($ naloxone vs 10791$)=7.95$ (Schild), 7.5 (constrained) see accompanying fig.

NALOXONE VERSUS NIH 10791 IN TAIL-FLICK Apparent pA2 (Schild)

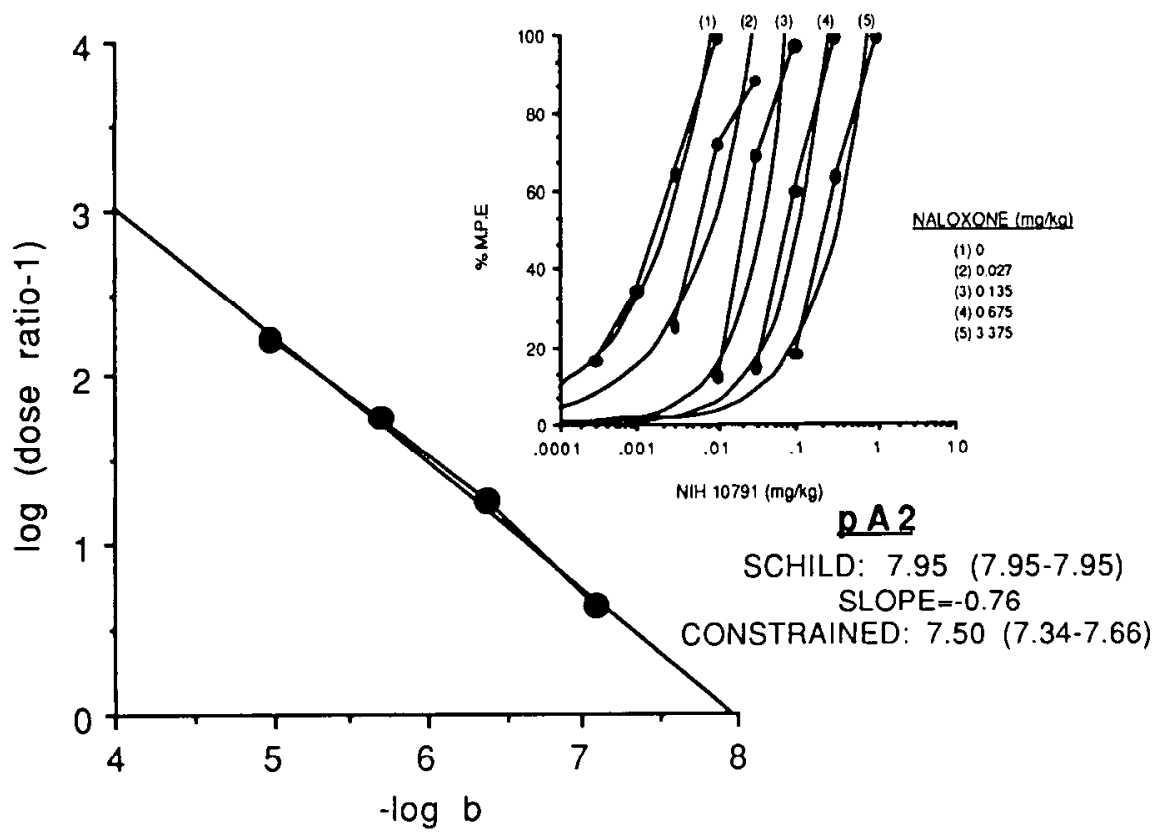


NIH $10791 \quad( \pm)-1-(2-H y d r o x y-2-p h e n y l e t h y l)-c-3-m e t h y l-4-[(1-$ oxopropyl)phenylamino]-r-4-piperidinecarboxylic acid methyl ester hydrochloride (continued)

\section{MONKEY DATA}

(SDS)

As shown in the fig., NIH 10791 dose-dependently substituted completely for morphine. Onset of action was rapid and duration of action was at least as long as that of morphine. Potency estimate: $6000 \mathrm{x}$ morphine.

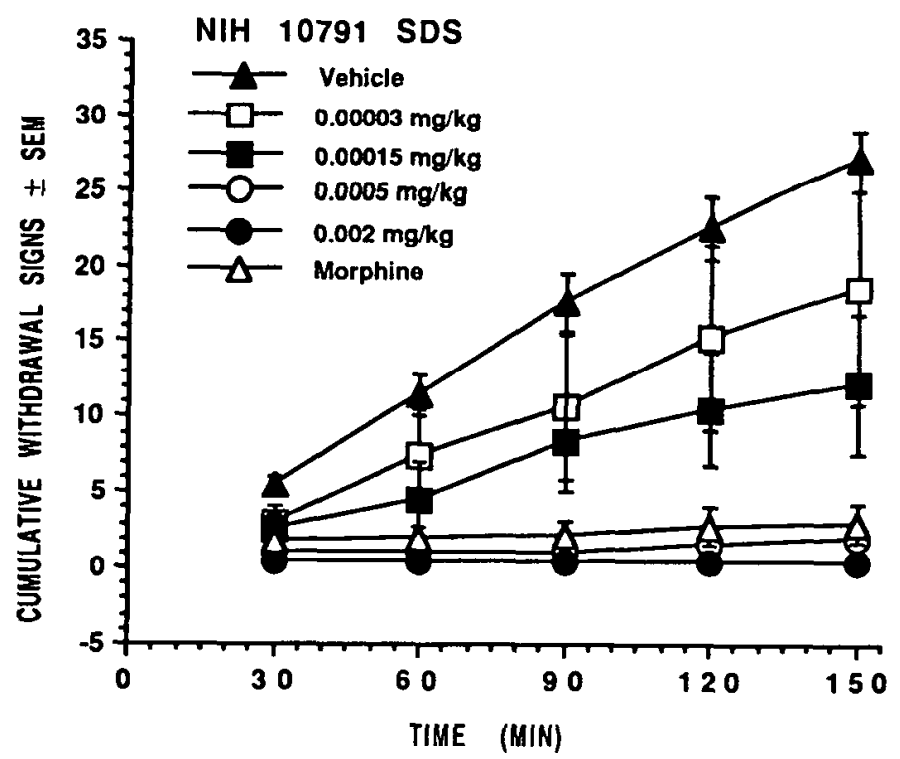

Comments: All the data suggested that NIH 10791 was a potent mu agonist. In addition, naloxone interacted competitively with this compound. 
NIH 10792 ( \pm )-1-(2-Hydroxy-2-phenylethyl)-t-3-methyl-t-[(1oxopropyl)phenylamino-r-4-piperidinecarboxylic acid ethyl ester hydrochloride

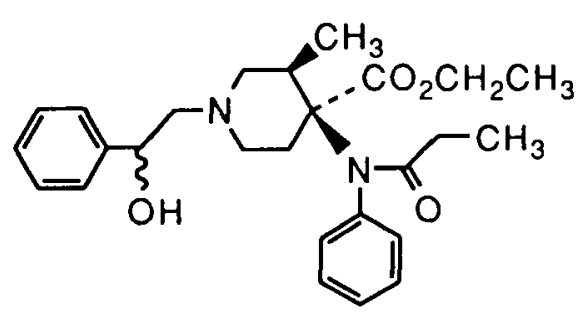

MOUSE DATA - ED50 OR AD50, $\mathrm{mg} / \mathrm{kg} \mathrm{95 \%} \mathrm{C.L.)} \mathrm{or} \mathrm{\%} \mathrm{change}$

1) $\mathrm{TF}-2.4 \times 10^{-4}\left(1.4 \times 10^{-4}-4.0 \times\right.$ $\left.10^{-4}\right)$

2) $\mathrm{TF}$ vs. $\mathrm{M}$ - Inactive at $1.0,10.0$ and $30.0^{\mathrm{a}}$

3) PPQ - $1.0 \times 10^{-4}\left(5.0 \times 10^{-5}-\right.$ $\left.21.0 \times 10^{-4}\right)$

4) HP $-0.003(0.001-0.009)^{\mathrm{a}}$

${ }^{\mathrm{a}}$ One mouse died at $30.0 \mathrm{mg} / \mathrm{kg}$

Special Test: Naloxone $\mathrm{AD}_{50}$ vs $\mathrm{ED}_{80}$ of $\mathrm{NIH} 10792$ in $\mathrm{TF}=0.04$ (0.01 0.12 )

\section{MONKEY DATA}

(SDS)

As shown in the accompanying fig., NIH 10792 substituted completely for morphine at 0.0001 and $0.0004 \mathrm{mg} / \mathrm{kg}$. Onset of action was similar to that of morphine, however, duration of action was shorter. Potency estimate is 30,000 $\mathrm{x}$ morphine. One monkey receiving the high dose was scratching. This sign is commonly seen either when morphine is given to non-dependent monkeys or when excessive doses are given to dependent monkeys.

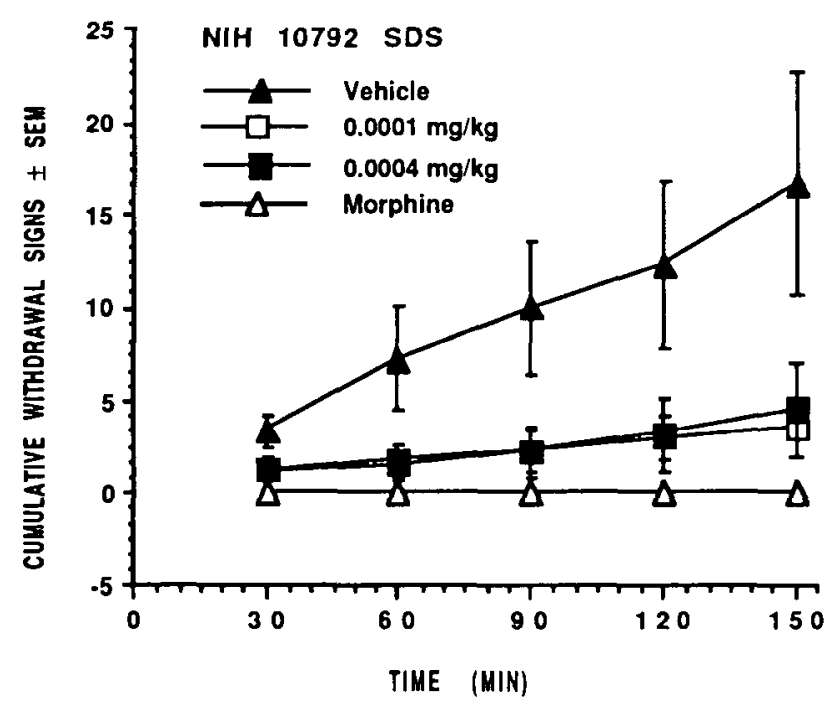

Comments:

The profile of activity indicates that NIH 10792 is an extremely potent mu agonist. 
NIH $10793 \quad( \pm)-\mathrm{N}-[1-(2-H y d r o x y-2-p h e n y l e t h y l)-4-m e t e t h y l-c-3-$ methyl-r-4-piperidinyl]-N-phenylpropanamide oxalate

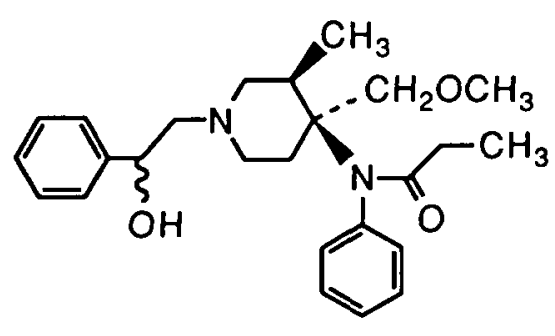

MOUSE DATA - ED50 OR AD50, $\mathrm{mg} / \mathrm{kg}(95 \%$ C.L.) or \% change

1) $\mathrm{TF}-0.0013(0.0004-0.004)^{\mathrm{a}}$

2) $\mathrm{TF}$ vs. $\mathrm{M}$ - Inactive at $1.0,10.0$ and $30.0^{\mathrm{b}}$

3) $P P Q-5 \times 10^{-4}(2.0 \times 10-4-1.9 \times$ $\left.10^{-3}\right)^{\mathrm{a}}$

4) $\mathrm{HP}-3.1 \times 10^{-4}\left(1.0 \times 10^{-4}-9.0 \times\right.$

${ }^{\mathrm{a}}$ Straub test and locomotor stimulation at $0.001,{ }^{\mathrm{b}}$ One mouse died at 10.0

Special Test: Naloxone $\mathrm{AD}_{50}$ vs $\mathrm{ED}_{80} \mathrm{NIH} 10793$ in $\mathrm{TF}=0.04(0.02-0.08)$

\section{MONKEY DATA}

(SDS)

NIH 10793 is approximately 10,000 times more potent than morphine. Onset of action is prompt and duration of action is less than that of morphine. At the high dose $(0.0006 \mathrm{mg} / \mathrm{kg})$ overt signs designated body sag, ataxia, and slowing were noted. The cummulative withdrawal scores were plotted for each observation period and are illustrated in the fig.

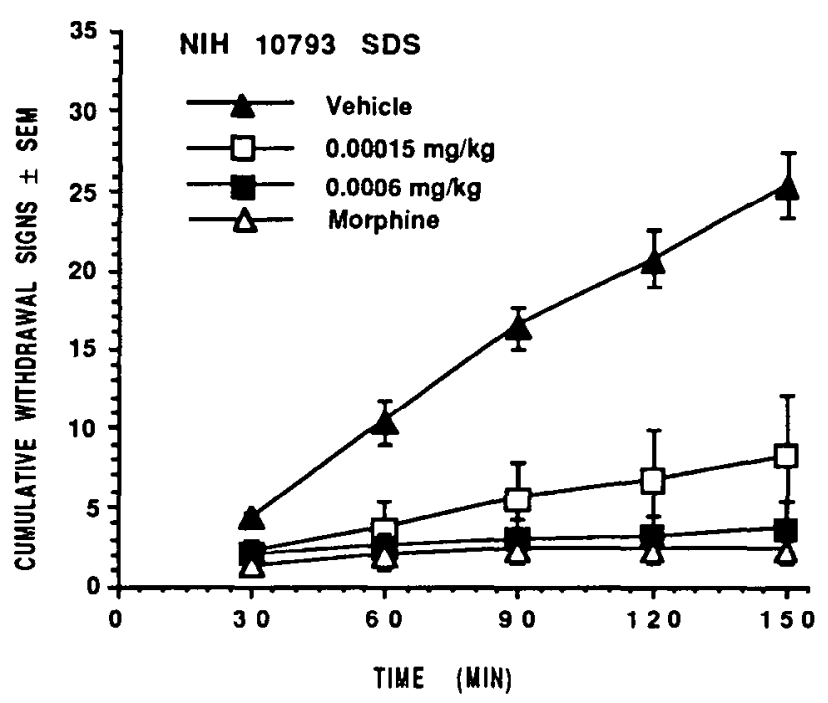

$\underline{\text { Comments: }}$

$\mathrm{NIH} 10793$ displays potent biological properties commonly associated with mu agonists. 


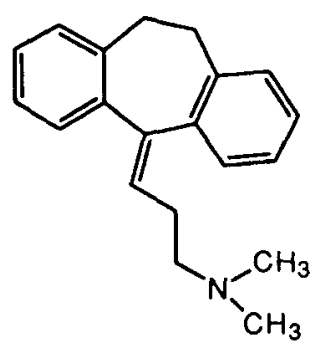

MOUSE DATA - ED50 OR AD50, $\mathrm{mg} / \mathrm{kg}$

(95\% C.L.) or \% change

1) $\mathrm{TF}-11 \%$ at $1.0,16 \%$ at 10.0 and $15 \%$ at 30.0

2) TP vs. M - Inactive at 1.0 and 30.0 and $20 \%$ at 10.0

3) PPQ $-0.09(0.03-0.31)$

4) $\mathrm{HP}-0 \%$ at $1.0,5 \%$ at 10.0 and $38 \%$ at $30.0^{\mathrm{a}}$

${ }^{\mathrm{a}}$ The mice had splayed limbs at 10.0 and $30.0 \mathrm{mg} / \mathrm{kg}$

Special Test: Naloxone Overt Behavior Challenge Tests

\section{A. $\quad \underline{\text { Reversal Test }}$}

Mice were injected s.c. with $30 \mathrm{mg} / \mathrm{kg}$ NIH 10794. Twenty m later they received $1.0 \mathrm{mg} / \mathrm{kg} / \mathrm{s} . c$. of naloxone and observed $10 \mathrm{~m}$ later. Naloxone did not abolish the sign designated splayed legs and eyelid ptosis.

\section{B. Prevention}

Mice received $1.0 \mathrm{mg} / \mathrm{kg} / \mathrm{s} . c$. of naloxone followed $10 \mathrm{~m}$ later by 30.0 $\mathrm{mg} / \mathrm{kg} / \mathrm{s}$.c. of $\mathrm{NIH} 10794$ and observed $20 \mathrm{~m}$ later. Naloxone did not prevent the emergence of the signs indicated above

Results: In both tests, naloxone failed to prevent or reverse the sign designated splayed legs or eyelid ptosis.

\section{Monkey}

\section{A. SDS}

Although the data in the accompanying group suggested that NIH 10794 was dose-dependently suppressing withdrawal, suppression was associated with head and body jerks, slowing, ataxia, or incoordination, jaw sag and eyelid ptosis. The neurological actions probably interfered with the expression of the withdrawal signs. 


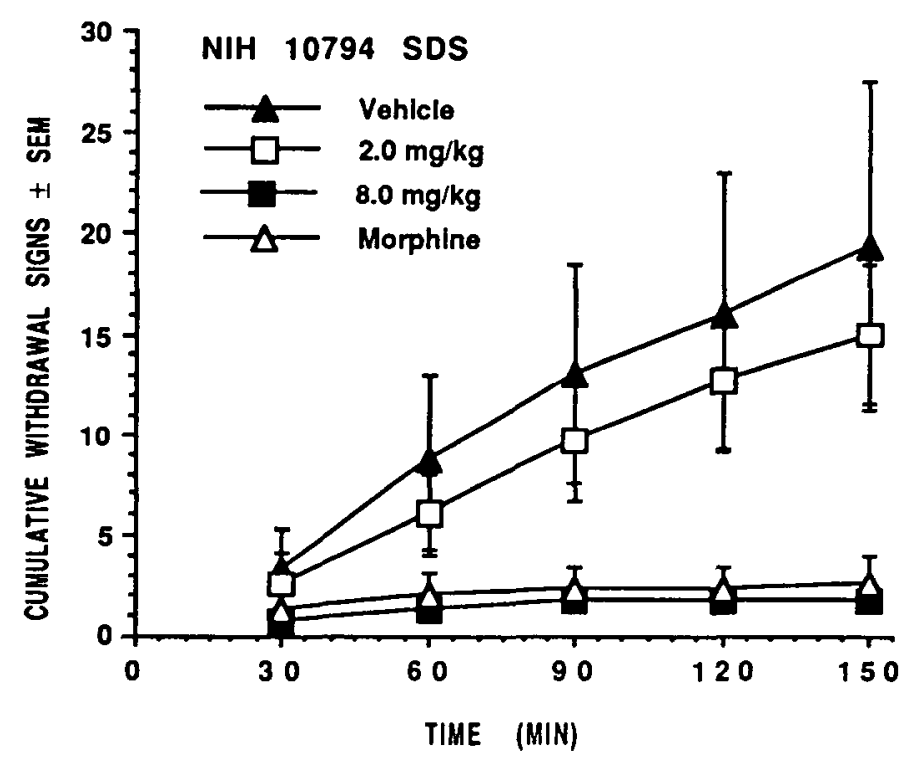

B. Non-Morphine-Dependent

In order to determine whether the overt neurological signs indicated above were associated with opioid properties, one monkey was given $\mathrm{NIH} \mathrm{10794,} \mathrm{at} \mathrm{15-m}$ intervals, $1.0 \mathrm{mg} / \mathrm{kg}, 2.0 \mathrm{mg} / \mathrm{kg}$ and $4.0 \mathrm{mg} / \mathrm{kg}$, respectively for a total dose of $7.0 \mathrm{mg} / \mathrm{kg}$. The signs designated slowing, body sag, ataxia (incoordination) and splayed legs were noted. The animal was given $1.0 \mathrm{mg} / \mathrm{kg} / \mathrm{s}$.c. of naloxone and observed for $90 \mathrm{~m}$. Naloxone failed to antagonize any and all of the signs observed.

\section{Comments:}

10794 produced effects in mice and monkeys which suggested antinociception and suppression of withdrawal. However, these effects were associated with neurological signs which were not antagonized by naloxone. We conclude that NIH 10794's actions are not associated with opiatergic mechanisms. 
NIH 10795 4-(3-Hydroxyphenyl-4-(1-oxopropyl)-1-(2-methyl-2butenyl)piperidine hydrochloride<smiles>CCOC(C)(c1cccc(O)c1)C1(CC)CCN(CC=C(C)C)CC1</smiles>

MOUSE DATA - ED50 OR AD50, $\mathrm{mg} / \mathrm{kg}$

(95\% C.L.) or \% change

1) $\mathrm{TF}-9.7(4.7-20.3)$

2) $\mathrm{TF}$ vs. $\mathrm{M}$ - Inactive at $1.0,10.0$ and 30.0

3) PPQ - $2.2(1.1-4.2)$

4) $\mathrm{HP}-8.3(3.7-19.0)$

\section{MONKEY DATA}

(SDS)

NIH partially suppressed withdrawal in a non-dose-related manner (see fig.). The suppression involved the signs designated lying on side or abdomen, fighting, and restlessness. However, at the high dose, during the first half-hour observation period, vomiting, coughing and frothy salivation was observed in one monkey and vomiting in another. These latter signs are sometimes seen during precipitated withdrawal and not during abrupt withdrawal. Perhaps slow onset of action confounded the interpretation.

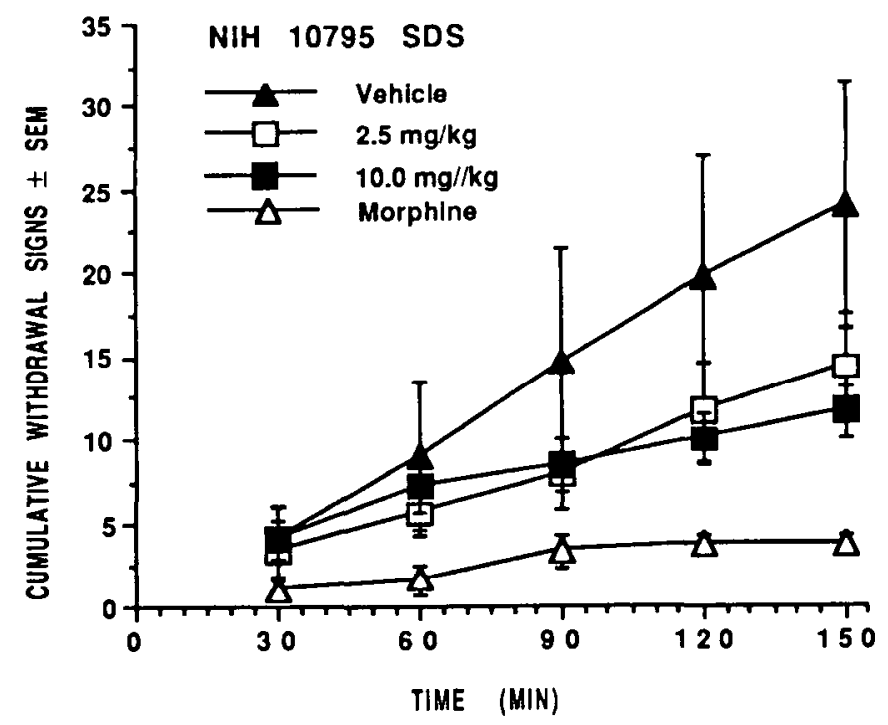

\section{Comments:}

The rodent data are typical of substances with opioid properties. However, the results in primates are not definitive and additional studies are indicated. 


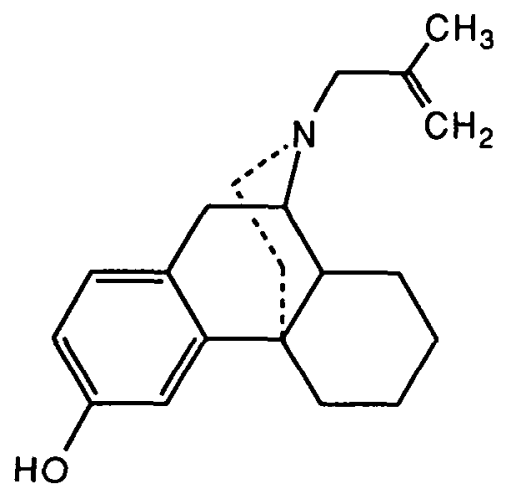

MOUSE DATA - ED50 OR AD50, $\mathrm{mg} / \mathrm{kg}$

(95\% C.L.) or \% change

1) $\mathrm{TF}-23 \%$ at 30.0 , Inactive at 1.0 and $10.0^{\mathrm{a}}$

2) $\mathrm{TF}$ vs. M - Inactive at $1.0,10.0$ and $30.0^{\mathrm{a}}$

3) $\mathrm{PPQ}-11.4(5.3-24.4)^{\mathrm{a}}$

4) $\mathrm{HP}-25 \%$ at 30.0 , Inactive at 1.0 and $10.0^{\mathrm{a}}$

${ }^{\mathrm{a}}$ Vehicle-10\% hydroxypropyl- $\beta$ cyclodextrin aqueous solution

\section{MONKEY DATA}

SDS

As shown in the fig., NIH 10796 partly attenuated withdrawal at $15 \mathrm{mg} / \mathrm{kg}$. Most of this action was due to a reduction in the signs designated retching and wet-dog. One monkey receiving the high dose convulsed. The convulsions were controlled with pentobarbital (30 mg i.p.). Vehicle was $25 \%$ hydroxypropyl-3--cyclodextrin aqueous solution.

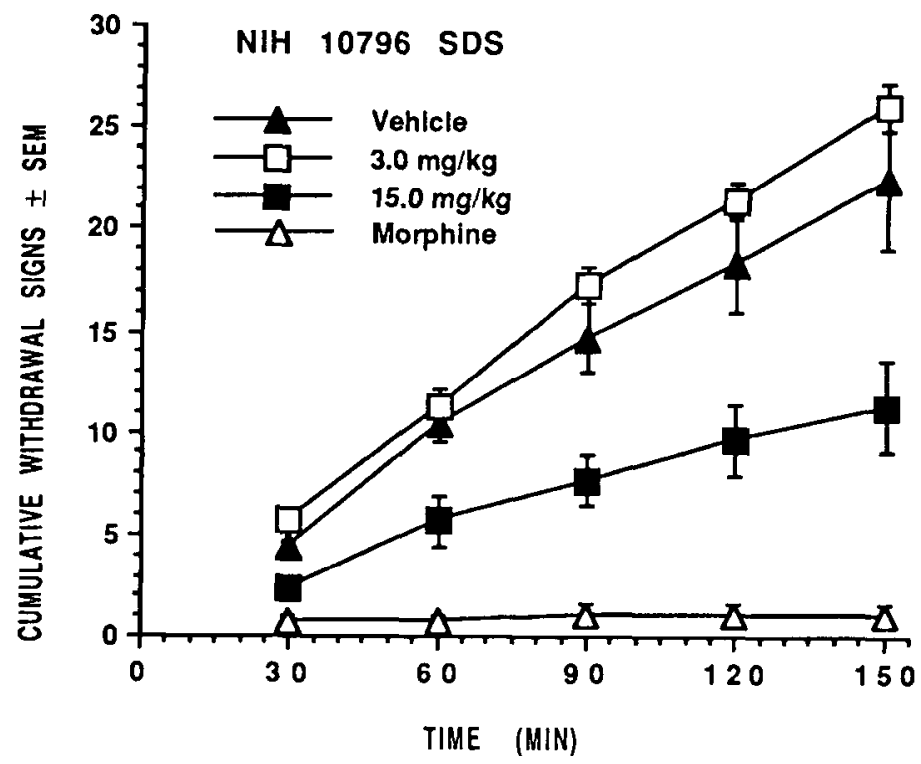

Comments:

NIH 10796 does not display salient mu opioid activity. However, the drug produced CNS excitation. 
NIH 10802 (-)-3-Hydroxy-N-(4-hydroxybenzyl)morphinan hydrobromide

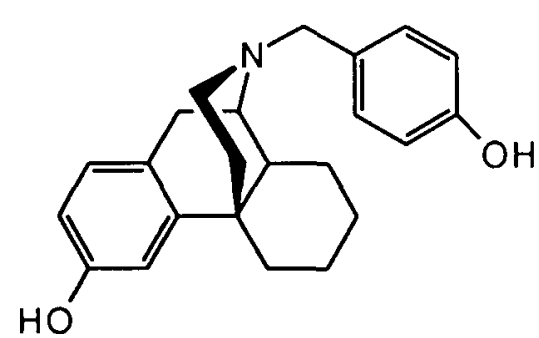

MOUSE DATA - $\mathrm{ED}_{50} \mathrm{OF} \mathrm{AD}_{50}$, $\mathrm{mg} / \mathrm{kg}$

(95\% C.L.) or \% change

1) $\mathrm{TF}$ - Inactive at 1.0, 10.0 and $30.0^{\mathrm{a}}$

2) $\mathrm{TF}$ vs. $\mathrm{M}$ - Inactive at $1.0,10.0$ and $30.0^{\mathrm{a}}$

3) PPQ - Inactive at $1.0,14 \%$ at 10.0 and $24 \%$ at $30.0^{\mathrm{a}}$

4) HP - Inactive at 1.0, 10.0 and $30.0^{\mathrm{a}}$

${ }^{a}$ Vehicle 5\% hydroxypropyl-3cyclodextrin in water.

\section{Comments:}

Antinociceptively, NIH 10802 is not remarkable.

NIH $10803( \pm)$-N-Allylmecamylamine $\bullet \mathrm{HCl}$

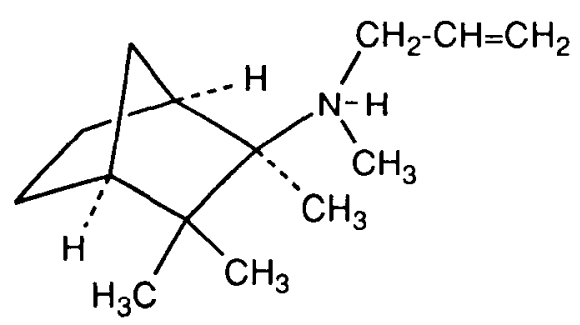

MOUSE DATA - ED50 OR AD50, $\mathrm{mg} / \mathrm{kg}$ (95\% C.L.) or \% change

1) $\mathrm{TF}$ - Inactive at 1.0, 10.0 and 30.0

2) $\mathrm{TF}$ vs. $\mathrm{M}$ - Inactive at $1.0,10.0$ and 30.0

3) PPQ - 9.8 (4.0 - 23.8)

4) $\mathrm{HP}$-Inactive at 1.0 and $10.0,25 \%$ at 30.0

\section{MONKEY DATA}

(SDS)

Although NIH 10803 appeared to partially suppress withdrawal (see fig.), this effect was accompanied by slowing and ataxia. One monkey receiving the high dose $(15 \mathrm{mg} / \mathrm{kg})$ also appeared very subdued and confused. It is concluded that the drug neither substituted for morphine nor exacerbated withdrawal. 
NIH $10803 \quad( \pm)$-N-Allylmecamylamine $\cdot \mathrm{HCl}$ (continued)

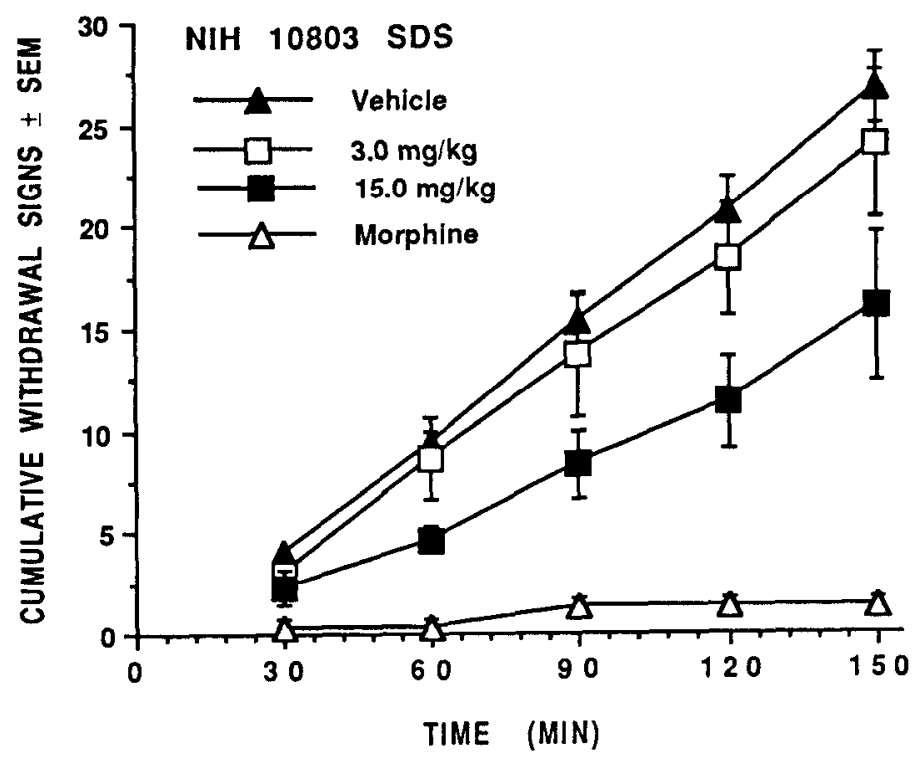

Comments:

NIH 10803 showed weak antinociceptive activity in the PPQ assay and had little effect on abstinence in morphine-dependent monkeys.

NIH 10805 1-Chlorodiprenorphine oxalate

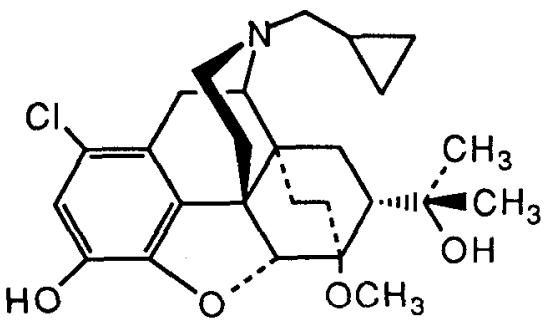

MOUSE DATA - ED50 OR AD50, $\mathrm{mg} / \mathrm{kg}$

(95\% C.L.) or \% change

1) $\mathrm{TF}$ - Inactive at $1.0,10.0$ and 30.0

2) TF vs. M - $0.09(0.03-0.3)$

3) PPQ - Inactive at $1.0,10.0$ and $14 \%$ at 30.0

4) $\mathrm{HP}$ - Inactive at 1.0, 10.0 and $25 \%$ at 30.0

5) $\quad \underline{\text { Apparent }} \mathrm{pA} 2 \underline{2}$ vs Morphine (mu agonist) in Tail-Flick Test

As shown in the fig., NIH 10805 behaved as a non-competitive antagonist. 
NIH 10805 1-Chlorodiprenorphine oxalate (cont.)

NIH 10805 VERSUS MORPHINE IN TAIL-FLICK

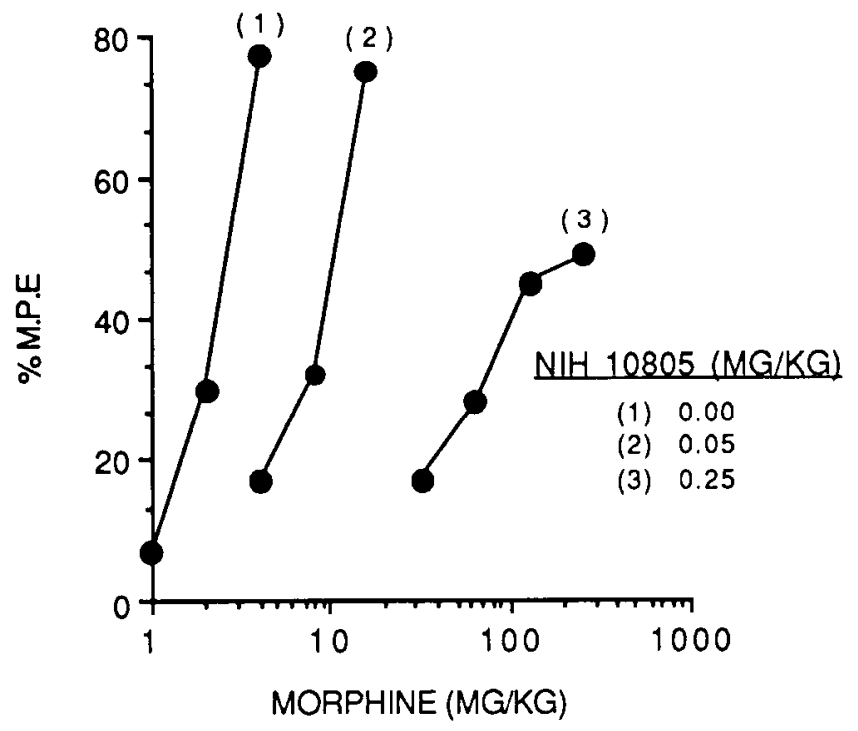

6) Apparent $\mathrm{pA}_{2}$ vs NIH 10672 (kappa agonist) in Tail-Flick Test.

An apparent $\mathrm{pA}_{2}$ of 8.0 was calculated for NIH 10672 (see accompanying fig.). The results indicate that NIH 10672 has a high affinity for the kappa receptor.

NIH 10805 VERSUS NIH 10672 IN TAIL-FLICK Apparent pA2 Schild

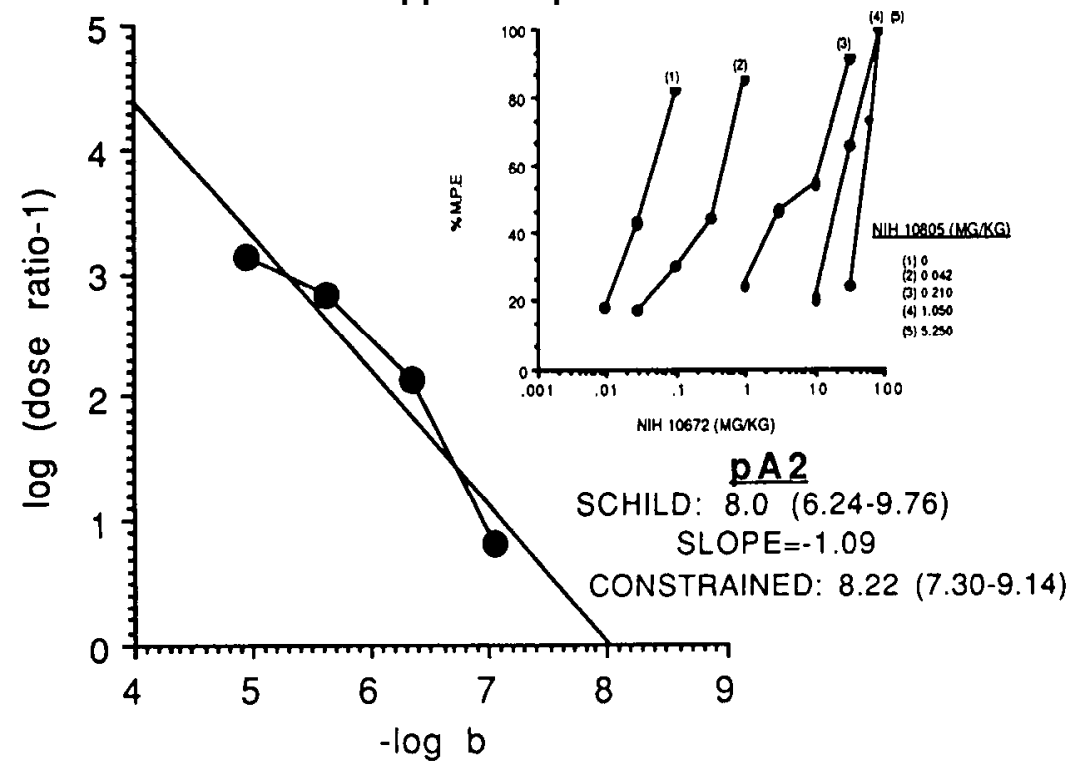


NIH 10805 1-Chlorodiprenorphine oxalate (continued)

7. Special Time-Course Study vs ED80 of morphine in Tail-Flick.

The results illustrated in the fig. suggest that NIH 10805 acts promptly and has a duration of action in excess of $4 \mathrm{hrs}$.

TIME COURSE OF NIH 10805 ANTAGONISM OF MORPHINEINDUCED ANTINOCICEPTION IN TAILFLICK TEST

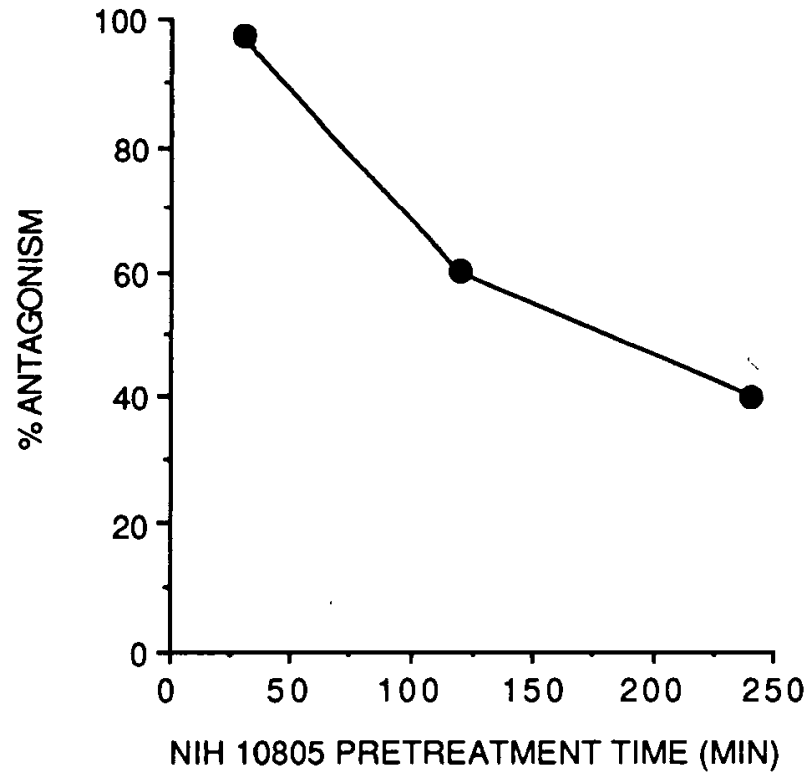

\section{MONKEY}

\section{1. $\quad$ Preliminary SDS}

At doses of $0.01,0.02$ and $0.02 \mathrm{mg} / \mathrm{kg}$ at $15 \mathrm{~m}$ intervals respectively, NIH 10805 exacerbated withdrawal. Two $3.0 \mathrm{mg} / \mathrm{kg}$ doses of morphine did not completely attenuate withdrawal.

\section{2. $\quad \underline{\text { PPt-W }}$}

NIH 10805 dose-dependently precipitated withdrawal in morphine-dependent monkeys. Onset and duration of action are similar to those of naloxone at equipotent doses. In addition, potency is estimated to be equivalent to that of naloxone. At the highest dose tested, some of the monkeys defecated, showed body jerks, ejaculated frequently and uttered clucking noises. 
NIH 10805 1-Chlorodiprenorphine oxalate (continued)

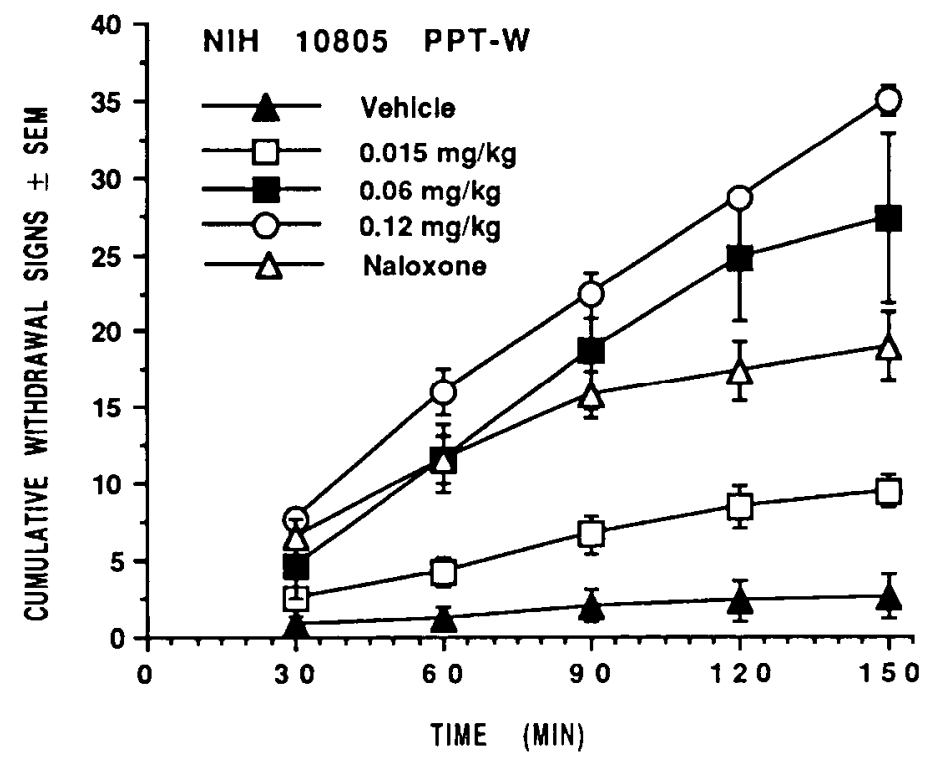

Comments:

NIH 10805 is a potent opioid antagonist with an unusual combination of mechanisms in the mouse; it behaved as a noncompetitive antagonist on mu receptors and as a competitive antagonist on kappa receptors. In the monkey studies, it can be said that NIH 10805 has mu antagonist properties. 
NIH 10809 N-Cyclopropylmethyl[7 $\boldsymbol{\alpha}, 8 \boldsymbol{\alpha}, 2^{\prime} 3^{\prime}$ ']cyclopentano-1'[R]hydroxy6,14-3-endoethenotetrahydronororipavine $\cdot \mathrm{HCl}$

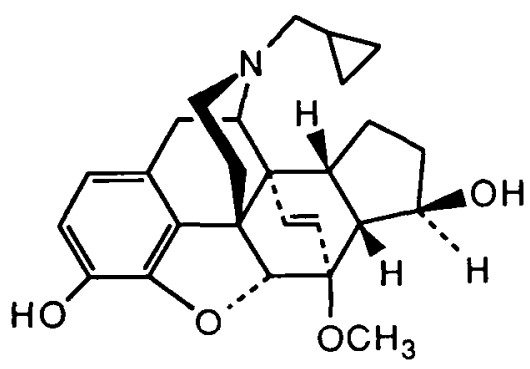

MOUSE DATA - ED50 OR AD50, $\mathrm{mg} / \mathrm{kg}$

(95\% C.L.) or \% change

1) $\mathrm{TF}-1) 0.1(0.03-0.34)$

2) $0.3(0.83-2.7)$

2) $\mathrm{TF}$ vs. $\mathrm{M}-9.3(3.6-23.9)$

$0.3(0.83-2.7)$

3) PPQ - $0.01(0.004-0.03)$

4) $\mathrm{HP}-1) 0.13 \%$ at $0.1,25 \%$ at 0.3 , $50 \%$ at $1.0,3.0,10.0$ and $30.0^{\mathrm{a}}$

2) $50 \%$ at $0.03,0.1$ and 1.0 , $25 \%$ at $3.0,38 \%$ at 10.0 and $63 \%$ at 30.0

${ }^{a}$ Moderate ataxia, copious urination. At the highest dose the mice were not ambulating.

\section{MONKEY DATA}

\section{A. (SDS)}

As shown in the graph, NIH 10809 appeared to exacerbate withdrawal. However, at doses of 0.05 and $0.025 \mathrm{mg} / \mathrm{kg}$, the results were not dose-related. In addition, a number of overt behavioral signs were noted including slowing, ataxia, jaw sag, piloerection, clucking sounds, severe tremors and body jerks. Perhaps lower doses should be tested to detect agonist activity. 


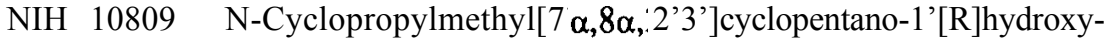
6,14-3-endoethenotetrahydronororipavine $\cdot \mathrm{HC1}$ (continued)

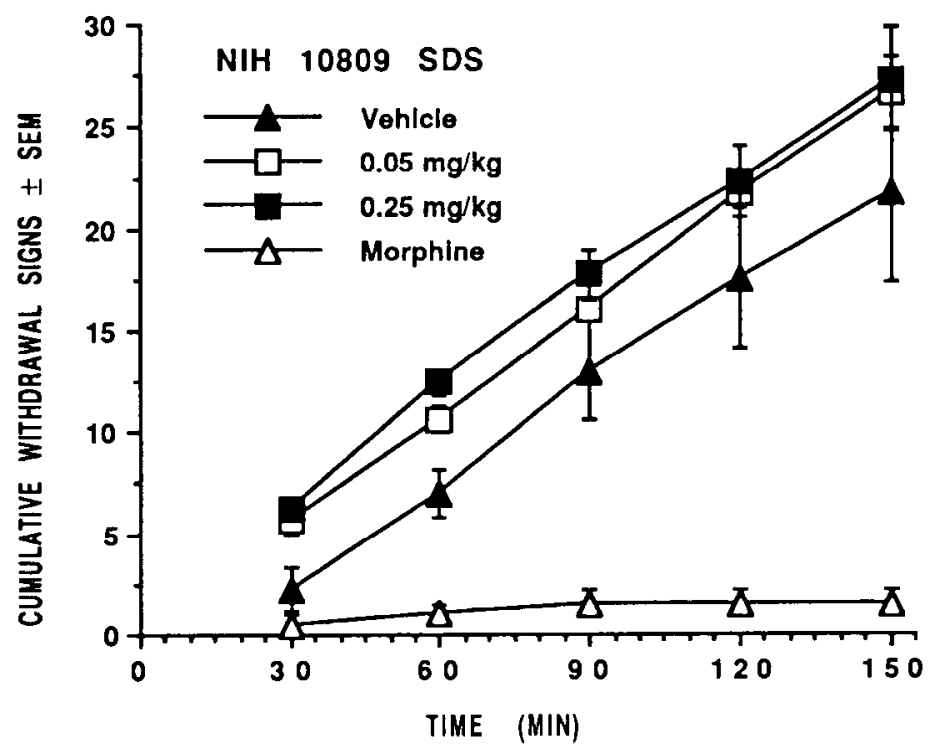

B. $(\mathrm{PPt}-\mathrm{W})$

As shown in the illustration, NIH 10809 precipitated withdrawal at doses of 0.05 and $0.25 \mathrm{mg} / \mathrm{kg}$. Whereas onset of action was as rapid as the control naloxone, duration of action was at least $150 \mathrm{~m}$; much longer than that of naloxone. Potency is estimated as equivalent to naloxone. Many of the monkeys receiving NIH 10809 made a clucking noise and vocalized. Profuse salivation, disorientation, slowing of long duration, and increased aggressive behavior towards handler were also noted in some monkeys. 
NIH 10809 N-Cyclopropylmethyl[7 $\alpha, 8 \alpha, 2^{\prime} 3^{\prime}$ ']cyclopentano-1'[R]hydroxy6,14-3-endoethenotetrahydronororipavine $\cdot \mathrm{HCl}$ (continued)

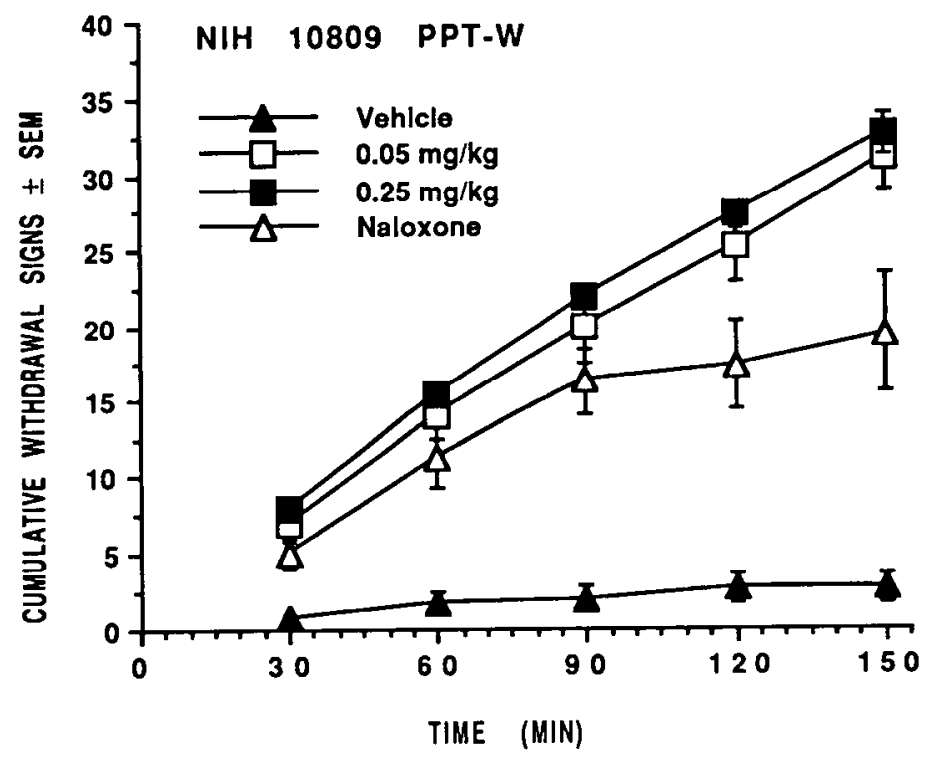

\section{Comments:}

The results in mice suggest that NIH 10809 is a partial opioid (mu) agonist. In the monkey, it manifests itself as a potent mu antagonist with a long duration of action. Some overt behavior also hint at kappa and sigma actions. 


\section{$\underline{\text { ACKNOWLEDGEMENTS }}$}

This study was supported by a contract (\#271-90-7200 and 3-8200) from the National Institute on Drug Abuse, Dr. Heinz Sorer, Contract Officer. We also acknowledge the expert assistance of Susan M. Tucker, and Larry Hughes. Special thanks to Laura Johnson for her help in the preparation of this manuscript using the Macintosh IIci.

\section{REFERENCES}

Aceto, M.D., Flora, R.E. and Harris, L.S. The effects of naloxone and nalorphine during the development of morphine dependence in rhesus monkeys. Pharmacol, 15: 1-9 1977.

Aceto, M.D., Flora, R.E. and Harris, L.S. Caffeine elicited withdrawal signs in morphine-dependent rhesus monkeys. Eur J Pharmacol, 50:203-207, 1978.

Aceto, M.D., McKean, D.B. and Pearl, J. Effects of opiates and opiate antagonists on the Straub tail reaction in mice. Br J Pharmacol, 36:225-239, 1969.

Atwell, L. and Jacobson, A.E. The search for less harmful analgesics. Lab Animal, 7:42-47, 1978.

D'Amour, F.E. and Smith, D.L. A method for determinating loss of pain sensation. J Pharmacol Exp Ther, 72:74-79, 1941.

Deneau, G.A. An analysis of factors influencing the development of physical dependence to narcotic analgesics in the rhesus monkey with methods for predicting physical dependence liability in man. Doctoral Dissertation, University of Michigan, 1956.

Dewey, W.L., Harris, L.S., Howes, J.F. and Nuite, J.A. The effects of various neurohumoral modulators on the activity of morphine and the narcotic antagonists in the tail-flick and phenylquinone tests. J Pharmacol Exp Ther, 175:435-442, 1970 .

Dewey, W.L. and Harris, L.S. Antinociceptive activity of the narcotic antagonists analogues and antagonistic activity of narcotic analgesics in rodents. J Pharmacol Exp Ther, 179:652-659, 1971.

Eddy, N.B. and Leimbach, D. Synthetic analgesics. II. Dithienylbutenyl- and dithienylbutylamines. J Pharmacol Exp Ther, 107:385-393, 1953.

Jacobson, A.E. and May, E.L. Structures related to morphine. XXXI. 2'Substituted benzomorphans. J Med Chem, 8:563-566, 1965.

Pearl, J. and Harris, L.S. Inhibition of writhing by narcotic antagonists. $\underline{\mathrm{J}}$ Pharmacol Exp Ther, 154,319, 1966.

Schild, M.O. $\mathrm{pA}_{2}$, A new scale for the measurement of drug antagonism. $\mathrm{Br} \mathrm{J}$ Pharmacol, 2: 189-206, 1947.

Seevers, M.H. Opiate addiction in the monkey. I. Methods of study. J. Pharmacol Exp Ther, 56:147-156, 1936. 
Seevers, M.H. and Deneau, G.A. Physiological aspects of tolerance and physical dependence. In: Root, W.S. and Hofman, F.G., eds. Physiological Pharmacology, Vol. I. New York: Academic Press, 1963. pp. 565-570.

Tallarida, R.J. and Murray, R.B. Manual of pharmacological calculations with computer programs. Second Edition: New York: Springer-Verlag, 1987. pp. 53-56.

Teiger, D.G. Induction of physical dependence on morphine, codeine, and meperidine in the rat by continuous infusion. J Pharmacol Exp Ther, 190:408415, 1974.

\section{AFFILIATION}

Department of Pharmacology and Toxicology, Medical College of Virginia, Virginia Commonwealth University, Richmond, VA 23298-0613 


\section{SUBJECT INDEX}

In order to simplify the Index, the subject subheadings along with page numbers can be found under both the chemical name and the NIH number.

(+)-4-[( $\alpha \mathrm{R})-\alpha(12 \mathrm{~S}, 5 \mathrm{R})-4-A l l y l-2,5-d i m e t h y l-1-p i p e r a z i n y l)-3-m e t h o x y b e n z y l]-$

N,N-diethylbenzamide (NIH 10815)

biological evaluation of physical-dependence potential and abuse liability,101

displacement of radiolabeled opioid binding, 158

inhibition of electrically stimulated mouse vas deferens, 158-159

$( \pm)$-N-Allylmecamylamine-hydrochloride (NIH 10803)

analgesia in mice, 203

biological evaluation of physical-dependence potential and abuse liability, 101

displacement of radiolabeled opioid binding, 150

inhibition of electrically stimulated mouse vas deferens, 151

physical dependence evaluation in rhesus monkeys, 203-204

Amitriptyline hydrochloride (NIH 10794)

analgesia in mice, 199

biological evaluation of physical-dependence potential and abuse liability, 101 physical dependence evaluation in rhesus monkeys, 199-200

(+)-N-Benzyl-3-hydroxymorphinan hydrochloride (NIH 10735)

analgesia in mice, 178

biological evaluation of physical-dependence potential and abuse liability, 95

physical dependence evaluation in rhesus monkeys, 178

Brain imaging

brain metabolism in substance abusers, 58

dopaminergic involvement in cocaine dependence, 60

EEG brain mapping of drug-induced intoxication, 59

fluoro-DOPA studies of human and animal cocaine abuse, 58-59

in drug-craving states, 61

spect imaging of dopamine transporter in cocaine dependence, 60

Bremazocine

displacement of $3 \mathrm{H}$-etorphine from rat brain membranes, 122

inhibition of sufentanil, DPDPE and U69,593 binding in rat and monkey, 123

inhibition of the mouse vas deferens preparation, 122

( \pm )-cis-N-[1-[1-(4-Bromophenyl)ethyl-2-hydroxy]-3-methyl-4-piperidyl]-N-

phenylpropanamide hydrochloride (NIH 10786)

analgesia in mice, 189

biological evaluation of physical-dependence potential and abuse liability, 99

displacement of radiolabeled opioid binding, 144

inhibition of electrically stimulated mouse vas deferens, 144

physical dependence evaluation in rhesus monkeys, 189

Buprenorphine

clinical pharmacology, 79-80

detoxification agent, $80-81$

improving treatment outcomes with behavioral treatment, 82

inhibition of sufentanil, DPDPE and U69,593 binding in rat and monkey, 123 multicenter study, effects of route of administration on outcome, 81

3-Carbamoyloxyecgonine methyl ester analogs probes for cocaine recognition site on dopamine transporter, 68

1-Chlorodiprenorphine oxalate (NIH 10805)

analgesia in mice, 204

biological evaluation of physical-dependence potential and abuse liability, 94 displacement of radiolabeled opioid binding, 151 
inhibition of electrically stimulated mouse vas deferens, 151-152

pA2 determinations in mouse tail flick, 204-206

physical dependence evaluation in rhesus monkeys, 206-207

2-(7-Chloro-1,8-haphthyridin-2-yl)-3-[(1,4-dioxa-8-azaspiro[4.5]dec-8-

yl)carbonylmethyl]isoindolin-1-one (CPDD 0038)

biological evaluation of physical-dependence potential and abuse liability, 102

effects on spontaneous activity and motor function in mice, 110-111

drug discrimination in rhesus monkeys, 110

reinforcing effects in rhesus monkeys, 110

1-Chloronaltrexone hydrochloride (NIH 10787)

analgesia in mice, 189

biological evaluation of physical-dependence potential and abuse liability, 93

displacement of radiolabeled opioid binding, 145

inhibition of electrically stimulated mouse vas deferens, 145

pA2 determinations in the mouse tail-flick procedure, 189-190

physical dependence evaluation in rhesus monkeys, 191-2

$\beta-\mathrm{CNA}$

inhibition of sufentanil, DPDPE and U69,593 binding in rat and monkey, 123

Cocaine

dopamine uptake inhibitors devoid of cocaine behavioral effects, 69-70

dopaminergic involvement in cocaine dependence, 60

fluoro-DOPA studies of human and animal cocaine abuse, 58-59

spect imaging of dopamine transporter in cocaine dependence, 60

probes for cocaine recognition site on dopamine transporter, 68

psychosocial treatments for abuse, 72-73

Codeine

analgesia in mice, 164

CPDD 0038 [2-(7-Chloro-1,8-haphthyridin-2-yl)-3-[(1,4-dioxa-8-

azaspiro[4.5]dec-8-yl)carbonylmethyl]isoindolin-1-one

biological evaluation of physical-dependence potential and abuse liability, 102

effects on spontaneous activity and motor function in mice, 110-111

drug discrimination in rhesus monkeys, 110

reinforcing effects in rhesus monkeys, 110

CPDD 0041 [Etryptamine acetate; $\boldsymbol{\alpha}$-Ethyl-1H-indole-3-ethanamine acetate; $\boldsymbol{\alpha}$ -

Ethyltryptamine acetate]

biological evaluation of physical-dependence potential and abuse liability, 102 drug discrimination in rhesus monkeys, 112-113

effects on spontaneous activity in mice, 113

infusion in pentobarbital-dependent rats, 114

reinforcing effects in rhesus monkeys, 111-112

substitution in rats chronically infused with cocaine, 115

(-)-Cyclazocine

analgesia in mice, 164

displacement of $3 \mathrm{H}$-etorphine from rat brain membranes, 122

inhibition of the mouse vas deferens preparation, 122

N-Cyclopropylmethyl[7 $\alpha, 8 \alpha, 2^{\prime}$ '']cyclopentano-1'[R]hydroxy-6,14-3-

endoethenotetrahydronororipavine hydrochloride (NIH 10809)

analgesia in mice, 208

biological evaluation of physical-dependence potential and abuse liability, 94

displacement of radiolabeled opioid binding, 152

inhibition of electrically stimulated mouse vas deferens, 153

physical dependence evaluation in rhesus monkeys, 208-210

N-Cyclopropylmethyl[7 $\alpha, 8 \alpha, 2$ ', 3'] cyclopentano-1'-[S]hydroxy-6,14-

endoethenotetrahydronororipavine hydrochloride (NIH 10810) 
biological evaluation of physical-dependence potential and abuse liability, 94 displacement of radiolabeled opioid binding, 153

inhibition of electrically stimulated mouse vas deferens, 154

N-Cyclopropylmethyl[7 $\left.\alpha, 8 \alpha, 2^{\prime}, 3^{\prime}\right]$ cyclohexano-1'-[S]hydroxy-6,14-

endoethenotetrahydronororipavine hydrochloride (NIH 10811)

biological evaluation of physical-dependence potential and abuse liability, 94 displacement of radiolabeled opioid binding, 154

DAMGO

inhibition of electrically stimulated mouse vas deferens, 155

displacement of $3 \mathrm{H}$-etorphine from rat brain membranes, 122

inhibition of sufentanil, DPDPE and U69,593 binding in rat and monkey, 123 inhibition of the mouse vas deferens preparation, 122

Dextrorphan Tartrate [NIH 04591; (+)-3-Hydroxy-N-methylmorphinan tartrate] analgesia in mice, 168

biological evaluation of physical-dependence potential and abuse liability, 95 displacement of 3H-etorphine from rat brain membranes, 122

inhibition of the mouse vas deferens preparation, 122

physical dependence evaluation in rats, 168

physical dependence evaluation in rhesus monkeys, 168

N-[(3,4-Dichlorophenyl)acetyl]-N,2-dimethyl-2-(N',N'-

dimethylamino)ethylamine oxalate (NIH 10703)

biological evaluation of physical-dependence potential and abuse liability, 100 displacement of radiolabeled opioid binding in monkey cortex, 136

(-)-5,9 $\alpha$-Dimethyl-2-ethyl-2'hydroxy-6,7-benzomorphan hydrochloride (NIH $10560)$

analgesia in mice, 170

biological evaluation of physical-dependence potential and abuse liability, 95 pA2 value versus morphine in mouse tail-flick, 171

physical dependence evaluation in rhesus monkeys, 171

(-)-5,9 $\alpha$-Dimelhyl-2-n -heptyl-2'-hydroxyl-6,7-benzomorphan hydrochloride

(NIH 10675)

analgesia in mice, 172

biological evaluation of physical-dependence potential and abuse liability, 96 physical dependence evaluation in rats, 172

physical dependence evaluation in rhesus monkeys, 172

(-)-5,9 $\alpha$-Dimethyl-2'-hydroxy-2- $n$-octyl-6,7-benzomorphan hydrochloride (NIH $10697)$

biological evaluation of physical-dependence potential and abuse liability, 96 displacement of radiolabeled opioid binding in monkey cortex, 133

(+)-5,9 $\alpha$-Dimethyl-2'-hydroxy-2- $n$-octyl-6,7-benzomorphan hydrochloride

(NIH 10698)

biological evaluation of physical-dependence potential and abuse liability, 96 displacement of radiolabeled opioid binding in monkey cortex, 134

2,3-Dimethyl-5-(3-hydroxyphenyl)morphan hydrochloride (NIH 10779) analgesia in mice, 184

biological evaluation of physical-dependence potential and abuse liability, 95 physical dependence evaluation in rhesus monkeys, 185

$3 \alpha$-Diphenylmethoxytropane analogs

dopamine uptake inhibitors devoid of cocaine behavioral effects, 69-70

DPDPE

displacement of $3 \mathrm{H}$-etorphine from rat brain membranes, 122

inhibition of the mouse vas deferens preparation, 122

Drug abuse 
affective disorders, 49-52

health of women, 45-48

historical summary of NIDA supported opioid research, 42-44

relationship of science and policy, 33-36

DSLET

displacement of $3 \mathrm{H}$-etorphine from rat brain membranes, 122

inhibition of sufentanil, DPDPE and U69,593 binding in rat and monkey, 123

inhibition of the mouse vas deferens preparation, 122

Environmental influence

determinants of neurochemical effects of drugs, 53-54

modulation of discriminative stimulus effects of drugs, 54-55

modulation of drug effects in drug abuse treatment, 56-57

modulation of reinforcing effects of drugs, 55-56

modulation of self-reporting effects of drugs, 56-57

$\alpha$-Ethyl-1H-indole-3-ethanamine acetate (CPDD 0041; Etryptamine acetate)

biological evaluation of physical-dependence potential and abuse liability, 102 drug discrimination in rhesus monkeys, 112-113

effects on spontaneous activity in mice, 113

infusion in pentobarbital-dependent rats, 114

reinforcing effects in rhesus monkeys, 111- 112

substitution in rats chronically infused with cocaine, 115

Ethylketazocine

displacement of $3 \mathrm{H}$-etorphine from rat brain membranes, 122

inhibition of the mouse vas deferens preparation, 122

Etorphine

displacement of $3 \mathrm{H}$-etorphine from rat brain membranes, 122

inhibition of sufentanil, DPDPE and U69,593 binding in rat and monkey, 123 inhibition of the mouse vas deferens preparation, 122

Fentanyl

displacement of $3 \mathrm{H}$-etorphine from rat brain membranes, 122

inhibition of the mouse vas deferens preparation, 122

1-(4-Fluorobenzyl)-4-(3-hydroxyphenyl)-4-(1-oxopropyl)piperidine

hydrochloride (NIH 10739)

analgesia in mice, 180

biological evaluation of physical-dependence potential and abuse liability, 97

displacement of radiolabeled opioid binding, 138

inhibition of electrically stimulated mouse vas deferens, 139

physical dependence evaluation in rhesus monkeys, 180

B-FNA

inhibition of sufentanil, DPDPE and U69,593 binding in rat and monkey, 123

Heroin

etiology, epidemiology and natural history of addiction, 74-78

(-)-3-Hydroxy-N-(4-hydroxybenzyl)morphinan hydrobromide (NIH 10802)

analgesia in mice, 203

biological evaluation of physical-dependence potential and abuse liability, 95

displacement of radiolabeled opioid binding, 149

inhibition of electrically stimulated mouse vas deferens, 150

14-Hydroxy-N-(2-methoxyethyl)-7,8-dihydronorisomorphine (NIH 10684) analgesia in mice, 176

analgesia in monkeys, 131

biological evaluation of physical-dependence potential and abuse liability, 92

displacement of radiolabeled opioid binding, 130

drug discrimination studies in rhesus monkeys, 131

inhibition of electrically stimulated mouse vas deferens, 130-131

physical dependence evaluation in rhesus monkeys, 175-177 
respiratory function studies in rhesus monkeys, 132

self-administration in rhesus monkeys, 132

14-Hydroxy-N-(2-methoxyethyl)-7,8-dihydronormorphine (NIH 10683)

analgesia in mice, 175

analgesia in monkeys, 129

biological evaluation of physical-dependence potential and abuse liability, 92

discriminative stimulus effects in rhesus monkeys, 128

displacement of radiolabeled opioid binding, 128

inhibition of electrically stimulated mouse vas deferens, 128

physical dependence evaluation in rhesus monkeys, 175

respiratory function studies in rhesus monkeys, 129

self-administration in rhesus monkeys, 129

(+)-3-Hydroxy-N-methylmorphinan tartrate [Dextrorphan Tartrate; NIH 04591] analgesia in mice, 168

biological evaluation of physical-dependence potential and abuse liability, 95

physical dependence evaluation in rats, 168

physical dependence evaluation in rhesus monkeys, 168

(+)-3-Hydroxy-N-(4-nitrobenzyl)morphinan oxalate (NIH 10749)

analgesia in mice, 182

biological evaluation of physical-dependence potential and abuse liability, 95

physical dependence evaluation in rhesus monkeys, 182

1-[1-(2-Hydroxyphenyl)cyclohexyl]-3,4-dehydropiperidine hydrochloride (NIH

10700)

biological evaluation of physical-dependence potential and abuse liability, 100 displacement of radiolabeled opioid binding in monkey cortex, 134

( \pm )-N-[1-(2-Hydroxy-2-phenylethyl)-4-methoxymethyl-c-3-methyl-r-4-

piperidinyl]-N-phenylpropanamide oxalate (NIH 10793)

analgesia in mice, 198

biological evaluation of physical-dependence potential and abuse liability, 99

displacement of radiolabeled opioid binding, 148

inhibition of electrically stimulated mouse vas deferens, 149

physical dependence evaluation in rhesus monkeys, 198

( \pm )-1-(2-Hydroxy-2-phenylethyl)-t-3-meLhyl-4-[(1-oxopropyl)phenylamino]-r-4-

piperidinecarboxamide methyl ester hydrochloride (NIH 10790)

analgesia in mice, 193

biological evaluation of physical-dependence potential and abuse liability, 99 displacement of radiolabeled opioid binding, 146

inhibition of electrically stimulated mouse vas deferens, 146

pA2 determinations in the mouse tail-flick procedure, 193

physical dependence evaluation in rhesus monkeys, 194

( \pm )-1-(2-Hydroxy-2-phenylethyl)-c-3-methyl-4-[(1-oxopropyl)phenylamino]-r-4-

piperidinecarboxylic acid methyl ester hydrochloride (NIH 10791)

analgesia in mice, 195

biological evaluation of physical-dependence potential and abuse liability, 99

displacement of radiolabeled opioid binding, 147

inhibition of electrically stimulated mouse vas deferens, 147

pA2 determinations in the mouse tail-flick procedure, 195

physical dependence evaluation in rhesus monkeys, 196

( \pm )-1-(2-Hydroxy-2-phenylethyl)-t-3-methyl-t-](1-oxopropyl)phenylamino-r-4-

piperidinecarboxylic acid ethyl ester hydrochloride (NIH 10792)

analgesia in mice, 197

biological evaluation of physical-dependence potential and abuse liability, 99

displacement of radiolabeled opioid binding, 148

inhibition of electrically stimulated mouse vas deferens, 148

physical dependence evaluation in rhesus monkeys, 197 
( \pm ) - cis-N-[1-(2-Hydroxy-2-phenylethyl)-3-methyl-4-piperidinyl]-N-(4-

fluorophenyl)propanamide hydrochloride (NIH 10785)

analgesia in mice, 188

biological evaluation of physical-dependence potential and abuse liability, 98

displacement of radiolabeled opioid binding, 143

inhibition of electrically stimulated mouse vas deferens, 143

physical dependence evaluation in rhesus monkeys, 188

( \pm )-cis-N-[1-(2-Hydroxy-2-phenylethyl)-3-methyl-4-piperidinyl]-N-(3-

fluorophenyl)propanamide hydrochloride (NIH 10784)

analgesia in mice, 186

biological evaluation of physical-dependence potential and abuse liability, 98 physical dependence evaluation in rhesus monkeys, 186

$(\beta ! S, 3 R, 4 S)-(+)-c i s-\mathrm{N}-[1-(2(\beta)-$-Hydroxy-2-phenylethyl)-3-methyl-4-

piperidinyl]-N-phenylpropanamide hydrochloride (NIH 10741)

analgesia in mice, 181

biological evaluation of physical-dependence potential and abuse liability, 98 naloxone $\mathrm{pA} 2$ value in the mouse tail-flick, 181

physical dependence evaluation in rhesus monkeys, 182

( \pm )-cis-N-[1-(2-Hydroxy-1-phenylethyl)-3-methyl-4-piperidinyl]-N-

phenylpropanamide hydrochloride (NIH 10765)

biological evaluation of physical-dependence potential and abuse liability, 98 displacement of radiolabeled opioid binding, 140

inhibition of electrically stimulated mouse vas deferens, 140-141

4-(3-Hydroxyphenyl)-1-(4-nitrobenzyl)-1-4-(1-oxopropyl) piperidine

hydrochloride (NIH 10738)

analgesia in mice, 179

biological evaluation of physical-dependence potential and abuse liability, 97

displacement of radiolabeled opioid binding, 137

inhibition of electrically stimulated mouse vas deferens, 138

physical dependence evaluation in rhesus monkeys, 179

4-(3-Hydroxyphenyl-4-(1-oxopropyl)-1-(2-methyl-2-butenyl)piperidine

hydrochloride (NIH 10795)

analgesia in mice, 201

biological evaluation of physical-dependence potential and abuse liability, 97

physical dependence evaluation in rhesus monkeys, 201

ICI 174,864

inhibition of sufentanil, DPDPE and U69,593 binding in rat and monkey, 123

$6 \boldsymbol{\alpha}$-Iodo-3,14-dihydroxy-17-cyclopropylmethyl-4,5 $\boldsymbol{\alpha}$-epoxymorphinan oxalate

(NIH 10702)

biological evaluation of physical-dependence potential and abuse liability, 92 displacement of radiolabeled opioid binding in monkey cortex, 136

$6 \beta$-Iodo-3,14-dihydroxy-17-cyclopropylmethyl-4,5 $\alpha$-epoxymorphinan oxalate

(NIH 10701)

biological evaluation of physical-dependence potential and abuse liability, 92 displacement of radiolabeled opioid binding in monkey cortex, 135

Joint probability

outcome index for pharmacology, 83

Ketazocine

displacement of $3 \mathrm{H}$-etorphine from rat brain membranes, 122

inhibition of the mouse vas deferens preparation, 122

Kleber, $\mathrm{H}$.

relationship of science to drug abuse policy, 33-36

Leshner, A.I.

drug abuse and addiction research, 6-15

Meperidine 
analgesia in mice, 164

cis-( \pm )-N-[3-Methyl-1-[2-oxo-2-(2-thienyl)ethyl]-4-piperidinyl]-N

phenylpropanamide hydrochloride (NIH 10762)

biological evaluation of physical-dependence potential and abuse liability, 98

displacement of radiolabeled opioid binding in monkey cortex, 139

inhibition of electrically stimulated mouse vas deferens, 140

3-(4-Methylpiperazinyliminomethyl)rifamycin, Rifampin or Rifampicin (NIH

10782)

analgesia in mice, 186

biological evaluation of physical-dependence potential and abuse liability, 100

displacement of radiolabeled opioid binding, 142

inhibition of electrically stimulated mouse vas deferens, 142

physical dependence evaluation in rhesus monkeys, 186

(+)-N-(2-Methylpropenyl)-3-hydroxymorphinan hydrochloride (NIH 10796) analgesia in mice, 202

biological evaluation of physical-dependence potential and abuse liability, 95 physical dependence evaluation in rhesus monkeys, 202

MK 801

structure-activity studies, 68069

Morphine

analgesia in mice, 164

displacement of $3 \mathrm{H}$-etorphine from rat brain membranes, 122

inhibition of sufentanil, DPDPE and U69,593 binding in rat and monkey, 123

inhibition of the mouse vas deferens preparation, 122

Morphine 3-acetate 6-sulfate zwitterion (NIH 10773)

analgesia in mice, 183

biological evaluation of physical-dependence potential and abuse liability, 93 displacement of radiolabeled opioid binding, 141

inhibition of electrically stimulated mouse vas deferens, 141

physical dependence evaluation in rhesus monkeys, 183-184

Nalorphine

analgesia in mice, 164

Naloxone

analgesia in mice, 164

inhibition of sufentanil, DPDPE and U69,593 binding in rat and monkey, 123

Naltrexone

analgesia in mice, 164

displacement of $3 \mathrm{H}$-etorphine from rat brain membranes, 122

inhibition of the mouse vas deferens preparation, 122

Nathan B. Eddy Memorial Award

introduction by J.V. Brady, 16-17

lecture by J.H. Jaffe, $18-32$

NIH 04591 [(+)-3-Hydroxy-N-methylmorphinan tartrate or Dextrorphan Tartrate] analgesia in mice, 168

biological evaluation of physical-dependence potential and abuse liability, 95 physical dependence evaluation in rats, 168

physical dependence evaluation in rhesus monkeys, 168

NIH 10560 [(-)-5,9 $\alpha$-Dimethyl-2-ethyl-2'hydroxy-6,7-benzomorphan

hydrochloride]

analgesia in mice, 170

biological evaluation of physical-dependence potential and abuse liability, 95

pA2 value versus morphine in mouse tail-flick, 171

physical dependence evaluation in rhesus monkeys, 171

NIH 10675 [(-)-5,9 $\alpha$--Dimethyl-2- n-heptyl-2'-hydroxyl-6,7-benzomorphan

hydrochloride] 
analgesia in mice, 172

biological evaluation of physical-dependence potential and abuse liability, 96 physical dependence evaluation in rats, 172

physical dependence evaluation in rhesus monkeys, 172

NIH 10683 [14-Hydroxy-N-(2-methoxyethyl)-7,8-dihydronormorphine]

analgesia in mice, 175

analgesia in monkeys, 129

biological evaluation of physical-dependence potential and abuse liability, 92 discriminative stimulus effects in rhesus monkeys, 128

displacement of radiolabeled opioid binding, 128

inhibition of electrically stimulated mouse vas deferens, 128

physical dependence evaluation in rhesus monkeys, 175

respiratory function studies in rhesus monkeys, 129

self-administration in rhesus monkeys, 129

$\mathrm{NIH} 10684$ [14-Hydroxy-N-(2-metoxyethyl)-7,8-dihydronorisomorphine]

analgesia in mice, 176

analgesia in monkeys, 131

biological evaluation of physical-dependence potential and abuse liability, 92

displacement of radiolabeled opioid binding, 130

drug discrimination studies in rhesus monkeys, 131

inhibition of electrically stimulated mouse vas deferens, 130-13 1

physical dependence evaluation in rhesus monkeys, 175-177

respiratory function studies in rhesus monkeys, 132

self-administration in rhesus monkeys, 132

NIH 10697 [(-)-5,9 $\boldsymbol{\alpha}$-Dimethyl-2'-hydroxy-2- $n$-octyl-6,7-benzomorphan

hydrochloride]

biological evaluation of physical-dependence potential and abuse liability, 96

displacement of radiolabeled opioid binding in monkey cortex, 133

NIH 10698 [(+)-5,9 $\alpha$-Dimethyl-2'-hydroxy-2- $n$-octyl-6,7-benzomorphan

hydrochloride]

biological evaluation of physical-dependence potential and abuse liability, 96 displacement of radiolabeled opioid binding in monkey cortex, 134

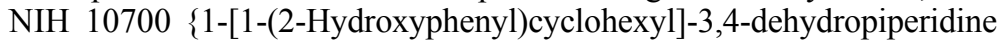

hydrochloride)

biological evaluation of physical-dependence potential and abuse liability, 100 displacement of radiolabeled opioid binding in monkey cortex, 134

NIH 10701 (6ß-Iodo-3,14-dihydroxy-17-cyclopropylmethyl-4,5 $\alpha$ epoxymorphinan oxalate)

biological evaluation of physical-dependence potential and abuse liability, 92 displacement of radiolabeled opioid binding in monkey cortex, 135

NIH 10702 ( $6 \alpha$-Iodo-3,14-dihydroxy-17-cyclopropylmethyl-4,5 $\alpha$ epoxymorphinan oxalate)

biological evaluation of physical-dependence potential and abuse liability, 92 displacement of radiolabeled opioid binding in monkey cortex, 136

NIH $10703\{\mathrm{~N}-[(3,4-\mathrm{Dichlorophenyl)acetyl]-N,2-dimethyl-2-(N',N'-}$

dimethylamino)ethylamine oxalate

biological evaluation of physical-dependence potential and abuse liability, 100 displacement of radiolabeled opioid binding in monkey cortex, 136

NIH 10705 [N-(n -Propyl)-N'-(3,4-dichlorophenylethyl)piperazine dhydrobromide]

biological evaluation of physical-dependence potential and abuse liability, 100 displacement of radiolabeled opioid binding in monkey cortex, 137

$\mathrm{NIH} 10735$ [(+)-N-Benzyl-3-hydroxymorphinan hydrochloride] analgesia in mice, 178 
biological evaluation of physical-dependence potential and abuse liability, 95 physical dependence evaluation in rhesus monkeys, 178

NIH 10738 [4-(3-Hydroxyphenyl)-1-(4-nitrobenzyl)-1-4-(1-oxopropyl)

piperidine hydrochloride]

analgesia in mice, 179

biological evaluation of physical-dependence potential and abuse liability, 97

displacement of radiolabeled opioid binding, 137

inhibition of electrically stimulated mouse vas deferens, 138

physical dependence evaluation in rhesus monkeys, 179

$\mathrm{NIH} 10739$ [1-(4-Fluorobenzyl)-4-(3-hydroxyphenyl)-4-(1-oxopropyl)piperidine

hydrochloride]

analgesia in mice, 180

biological evaluation of physical-dependence potential and abuse liability, 97

displacement of radiolabeled opioid binding, 138

inhibition of electrically stimulated mouse vas deferens, 139

physical dependence evaluation in rhesus monkeys, 180

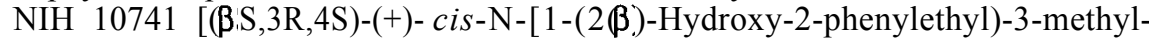

4-piperidinyl]-N-phenylpropanamide hydrochloride]

analgesia in mice, 181

biological evaluation of physical-dependence potential and abuse liability, 98

naloxone $\mathrm{pA} 2$ value in the mouse tail-flick, 181

physical dependence evaluation in rhesus monkeys, 182

NIH 10749 [(+)-3-Hydroxy-N-(4-nitrobenzyl)morphinan oxalate]

analgesia in mice, 182

biological evaluation of physical-dependence potential and abuse liability, 95 physical dependence evaluation in rhesus monkeys, 182

NIH 10762 \{cis-( \pm )-N-[3-Methyl-1-[2-oxo-2-(2-thienyl)ethyl]-4-piperidinyl]-N phenylpropanamide hydrochloride $\}$

biological evaluation of physical-dependence potential and abuse liability, 98 displacement of radiolabeled opioid binding in monkey cortex, 139

inhibition of electrically stimulated mouse vas deferens, 140

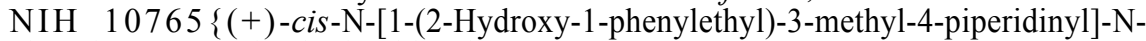
phenylpropanamide hydrochloride $\}$

biological evaluation of physical-dependence potential and abuse liability, 98

displacement of radiolabeled opioid binding, 140

inhibition of electrically stimulated mouse vas deferens, 140-141

NIH 10773 (Morphine 3-acetate 6-sulfate zwitterion)

analgesia in mice, 183

biological evaluation of physical-dependence potential and abuse liability, 93

displacement of radiolabeled opioid binding, 141

inhibition of electrically stimulated mouse vas deferens, 141

physical dependence evaluation in rhesus monkeys, 183-184

NIH 10779 [2,3-Dimethyl-5-(3-hydroxyphenyl)morphan hydrochloride] analgesia in mice, 184

biological evaluation of physical-dependence potential and abuse liability, 95 physical dependence evaluation in rhesus monkeys, 185

NIH 10782 [3-(4-Methylpiperazinyliminomethyl)rifamycin, Rifampin or

Rifampicin]

analgesia in mice, 186

biological evaluation of physical-dependence potential and abuse liability, 100 displacement of radiolabeled opioid binding, 142

inhibition of electrically stimulated mouse vas deferens, 142

physical dependence evaluation in rhesus monkeys, 186

$\mathrm{NIH} 10784$ [( \pm )-cis-N-[1-(2-Hydroxy-2-phenylethyl)-3-methyl-4-piperidinyl]-N-

(3-fluorophenyl)propanamide hydrochloride]

analgesia in mice, 186 
biological evaluation of physical-dependence potential and abuse liability, 98 physical dependence evaluation in rhesus monkeys, 186

$\mathrm{NIH} 10785$ [( \pm )-cis-N-[1-(2-Hydroxy-2-phenylethyl)-3-methyl-4-piperidinyl]-N(4-fluorophenyl)propanamide hydrochloride]

analgesia in mice, 188

biological evaluation of physical-dependence potential and abuse liability, 98 displacement of radiolabeled opioid binding, 143

inhibition of electrically stimulated mouse vas deferens, 143

physical dependence evaluation in rhesus monkeys, 188

$\mathrm{NIH} 10786$ [( \pm )-cis-N-[1-[1-(4-Bromophenyl)ethyl-2-hydroxy]-3-methyl-4-

piperidyl]-N-phenylpropanamide-hydrochloride]

analgesia in mice, 189

biological evaluation of physical-dependence potential and abuse liability, 99

displacement of radiolabeled opioid binding, 144

inhibition of electrically stimulated mouse vas deferens, 144

physical dependence evaluation in rhesus monkeys, 189

NIH 10787 [1-Chloronaltrexone•hydrochloride]

analgesia in mice, 189

biological evaluation of physical-dependence potential and abuse liability, 93 displacement of radiolabeled opioid binding, 145

inhibition of electrically stimulated mouse vas deferens, 145

pA2 determinations in the mouse tail-flick procedure, 189-190

physical dependence evaluation in rhesus monkeys, 191-192

NIH 10790 [( \pm )-1-(2-Hydroxy-2-phenylethyl)-t-3-methyl-4-[(1-

oxopropyl)phenylamino]-r-4-piperidinecarboxamide methyl ester hydrochloride] analgesia in mice, 193

biological evaluation of physical-dependence potential and abuse liability, 99 displacement of radiolabeled opioid binding, 146

inhibition of electrically stimulated mouse vas deferens, 146

pA2 determinations in the mouse tail-flick procedure, 193

physical dependence evaluation in rhesus monkeys, 194

NIH 10791 [( \pm )-1-(2-Hydroxy-2-phenylethyl)-c-3-methyl-4-[(1-

oxopropyl)phenylamino]-r-4-piperidinecarboxylic acid methyl ester

hydrochloride]

analgesia in mice, 195

biological evaluation of physical-dependence potential and abuse liability, 99 displacement of radiolabeled opioid binding, 147

inhibition of electrically stimulated mouse vas deferens, 147

pA2 determinations in the mouse tail-flick procedure, 195

physical dependence evaluation in rhesus monkeys, 196

NIH 10792 [(+)-1-(2-Hydroxy-2-phenylethyl)-t-3-metyl-t-[(1-

oxopropyl)phenylamino-r-4-piperidinecarboxylic acid ethyl ester hydrochloride] analgesia in mice, 197

biological evaluation of physical-dependence potential and abuse liability, 99 displacement of radiolabeled opioid binding, 148

inhibition of electrically stimulated mouse vas deferens, 148

physical dependence evaluation in rhesus monkeys, 197

$\mathrm{NIH} 10793[( \pm)-\mathrm{N}-[1-(2-H y d r o x y-2-p h e n y l e t h y l)-4-m e t h o x y m e t h y l-c-3-m e t h y l-$ r-4-piperidinyl]-N-phenylpropanamide oxalate]

analgesia in mice, 198

biological evaluation of physical-dependence potential and abuse liability, 99

displacement of radiolabeled opioid binding, 148

inhibition of electrically stimulated mouse vas deferens, 149

physical dependence evaluation in rhesus monkeys, 198

NIH 10794 (Amitriptyline hydrochloride) 
analgesia in mice, 199

biological evaluation of physical-dependence potential and abuse liability, 101 physical dependence evaluation in rhesus monkeys, 199-200

NIH 10795 [4-(3-Hydroxyphenyl-4-(1-oxopropyl)-1-(2-methyl-2-

butenyl)piperidine hydrochloride]

anlgesia in mice, 201

biological evaluation of physical-dependence potential and abuse liability, 97 physical dependence evaluation in rhesus monkeys, 201

MH 10796 [(+)-N-(2-Methylpropenyl)-3-hydroxymorphinan hydrochloride] analgesia in mice, 202

biological evaluation of physical-dependence potential and abuse liability, 95 physical dependence evaluation in rhesus monkeys, 202

$\mathrm{NIH} 10802$ [(-)-3-Hydroxy-N-(4-hydroxybenzyl)morphinan hydrobromide] analgesia in mice, 203

biological evaluation of physical-dependence potential and abuse liability, 95 displacement of radiolabeled opioid binding, 149

inhibition of electrically stimulated mouse vas deferens, 150

$\mathrm{NIH} 10803$ [( \pm -N-Allylmecamylamine•hydrochloride]

analgesia in mice, 203

biological evaluation of physical-dependence potential and abuse liability, 101 displacement of radiolabeled opioid binding, 150

inhibition of electrically stimulated mouse vas deferens, 151

physical dependence evaluation in rhesus monkeys, 203-204

$\mathrm{NIH} 10805$ [1-Chlorodiprenorphine oxalate]

analgesia in mice, 204

biological evaluation of physical-dependence potential and abuse liability, 94 displacement of radiolabeled opioid binding, 151

inhibition of electrically stimulated mouse vas deferens, 151-152

pA2 determinations in mouse tail flick, 204-206

physical dependence evaluation in rhesus monkeys, 206-207

NIH 10809 \{N-Cyclopropylmethyl[7 $\alpha, 8 \alpha, 22^{\prime} 3$ '] cyclopentano-1'[R]hydroxy-

6,14-3-endoethenotetrahydronororipavine hydrochloride)

analgesia in mice, 208

biological evaluation of physical-dependence potential and abuse liability, 94 displacement of radiolabeled opioid binding, 152

inhibition of electrically stimulated mouse vas deferens, 153

physical dependence evaluation in rhesus monkeys, 208-210

NIH 10810 (N-Cyclopropylmethyl[7 $\alpha, 8 \alpha, 2^{\prime}, 3^{\prime}$ ']cyclopentano-1'-[S]hydroxy-

6,14-endoethenotetrahydronororipavine hydrochloride)

biological evaluation of physical-dependence potential and abuse liability, 94 displacement of radiolabeled opioid binding, 153

inhibition of electrically stimulated mouse vas deferens, 154

NIH $10811\{$ N-Cyclopropylmethyl[7 $\alpha, 8 \alpha, 2$ ',3']cyclohexano-1'-[S]hydroxy-

6,14-endoethenotetrahydronororipavine hydrochloride\}

biological evaluation of physical-dependence potential and abuse liability, 94

displacement of radiolabeled opioid binding, 154

inhibition of electrically stimulated mouse vas deferens, 155

$\mathrm{NIH} 10812$ (2-Nitrobuprenorphine hydrochloride)

biological evaluation of physical-dependence potential and abuse liability, 94 displacement of radiolabeled opioid binding, 155

inhibition of electrically stimulated mouse vas deferens, 155-156

$\mathrm{NIH} 10813$ (2-Nitrodiprenorphine hydrochloride)

biological evaluation of physical-dependence potential and abuse liability, 94 displacement of radiolabeled opioid binding, 156

inhibition of electrically stimulated mouse vas deferens, 156-157 
NIH 10814 (2-Nitronaltrexone hydrochloride)

biological evaluation of physical-dependence potential and abuse liability, 93 displacement of radiolabeled opioid binding, 157

inhibition of electrically stimulated mouse vas deferens, 157-158

$\mathrm{NIH} 10815\{(+) 4-[(\alpha \cdot \mathrm{R})-\boldsymbol{\alpha}(12 \mathrm{~S}, 5 \mathrm{R})-4-A l l y l-2,5-d i m e t h y l-1-p i p e r a z i n y l)-3-$

methoxybenzyl]-N,N-diethylbenzamide\}

biological evaluation of physical-dependence potential and abuse liability, 101 displacement of radiolabeled opioid binding, 158

inhibition of electrically stimulated mouse vas deferens, 158-159

2-Nitrobuprenorphine hydrochloride (NIH 10812)

biological evaluation of physical-dependence potential and abuse liability, 94 displacement of radiolabeled opioid binding, 155

inhibition of electrically stimulated mouse vas deferens, 155-156

2-Nitrodiprenorphine hydrochloride (NIH 108 13)

biological evaluation of physical-dependence potential and abuse liability, 94

displacement of radiolabeled opioid binding, 156

inhibition of electrically stimulated mouse vas deferens, 156-157

2-Nitronaltrexone hydrochloride (NIH 10814)

biological evaluation of physical-dependence potential and abuse liability, 93

displacement of radiolabeled opioid binding, 157

inhibition of electrically stimulated mouse vas deferens, 157-158

Opioid

analgesics to treatment drugs, 67

Opioid receptors

characterization at the molecular level, 37

development of selective nonpeptide $\delta$ ligands, 66

genomic structure and analysis of promotor sequence of $\mu$ receptor gene, 39

human receptors and their genes, 38

interaction of selective nonpeptide ligands, 65-66

mapping and modulation of receptor gene expression, 40

molecular cloning and cellular function, 38-39

quantitation and localization of receptor gene expression, 41

Pentazocine

analgesia in mice, 164

President's comments

by George E. Bigelow, 1-5

$\mathrm{N}-(n$-Propyl)-N'-(3,4-dichlorophenylethyl)piperazine dhydrobromide (NIH

10705)

biological evaluation of physical-dependence potential and abuse liability, 100 displacement of radiolabeled opioid binding in monkey cortex, 137

Robinson, F.M.

memorial symposium, 63-64

(-)-SDF 10047

displacement of $3 \mathrm{H}$-etorphine from rat brain membranes, 122

inhibition of the mouse vas deferens preparation, 122

Service

effects on drug abuse treatment outcome, 71-72

psychosocial services during substance abuse treatment, 73

psychosocial treatments for abuse, 72-73

Simon, E.J.

historical summary of MDA supported opioid research, 42-44

Sufentanil

displacement of $3 \mathrm{H}$-etorphine from rat brain membranes, 122

inhibition of sufentanil, DPDPE and U69,593 binding in rat and monkey, 123

inhibition of the mouse vas deferens preparation, 122 


\section{Superfit}

inhibition of sufentanil, DPDPE and U69,593 binding in rat and monkey, 123 Treatment effects of amount of service on outcome, 71-72 psychosocial services during substance abuse treatment, 73 psychosocial treatments for drug abuse, 72-73

U50,488

displacement of $3 \mathrm{H}$-etorphine from rat brain membranes, 122 inhibition of mouse vas deferens preparation, 122 inhibition of sufentanil, DPDPE and U69,593 binding in rat and monkey, 123 U69,593

inhibition of sufentanil, DPDPE and U69,593 binding in rat and monkey, 123 UM $10712 \mathrm{R}$ displacement of $3 \mathrm{H}$-etorphine from rat brain membranes, 122 Women drug abuse and health, $45-48$ 
Aceto, Mario D.; 162

Amass, Leslie; 53, 82

Anderson, Paul S.; 63, 65

Augustin, Lance B.; 37

Ball, J. C.; 74

Bickel, Warren K.; 82

Bigelow, George E.; 1, 79

Bowman, Edward R.; 162

Brady, J. V.; 16

Bush, C. R.; 53

Calderon, Silvia; 65

Caudill, B. D.; 72

Childress, Anna Rose; 58

Clayton, R. C.; 74

Compton, M. A.; 83

Cottler, Linda B.; 45

Craddock, S. G.; 71

Dunteman, G.; 71

English, J. A.; 105

Etheridge, R. M.; 71

Evans, Chris; 37

Felsheim, Roderick F.; 37

Finnegan, Loretta P.; 45

Flynn, P. M.; 72

Foltin, R. W.; 53

France, C. P.; 117

Frank, D. A.; 45

Fuchs, James A.; 37

Gardner, E.; 49

Gatley, John; 60

Goodwin, F.; 49
Groves, G. A.; 74

Hagan, T. A.; 74

Harris, Louis S.; 105, 162

Haverkos, H. W.; 45

Hawkins, W. T.; 105

Higgins, Stephen T.; 82

Hill, J. L.; 81

Hoffman, J. A.; 72

Hubbard, R. L.; 71

Imai, Yasuo; 37

Inturrisi, Charles E.; 37

Jacobson, Arthur; 84

Jaffe, J. H.; 18

Johnson, Peter; 37

Johnson, R. E.; 80

Kamien, Jonathan B.; 53

Kleber, H.; 33

Kleiman, M.; 33

Klett, C. J.; 83

Kline, Richard H.; 65

Koman, III, J. J.; 72

Kosten, T. R.; 49

Kreek, Mary Jeanne; 37, 45

Lerner, M.; 74

Leshner, Alan I.; 6

Lewis, John; 65

Ling, Walter; 83

Loh, Horace H.; 37

London, Edythe; 58

Luckey, J. W.; 72

Lukas, Scott; 58 
Malison, Robert; 60

Markou, A.; 49

Massey, B. W.; 105

May, Everette L.; 162

Mayo, D. W.; 72

McDaniel, R. E.; 105

McLellan, A. T.; 73

Medizhradsky, F.; 117

Melega, William; 58

Mello, Nancy K.; 45

Min, Bon H.; 37

Moriwaki, Akiyoshi; 37

Nader, Michael A.; 53, 105

Newman, Amy Hauck; 65

Nurco, D. N.; 74

O'Brien, Charles P.; 58

Patrick, G. A.; 105

Pickens, R.; 49

Portoghese, Philip S.; 65

Rice, Kenner C.; 65

Rowlett, J. K.; 105

Rush, Craig R.

Schuh, K. J.; 79

Segal, D. L.; 81

Simon, Eric J.; 37, 42

Sloboda, Z.; 45

Smith, C. B.; 117

Solarz, A.; 33

Stitzer, M. L.; 79

Strain, Eric C.; 79

Uhl, George; 37

Vrana, Sheila L.; 53

Walsh, S. L.; 79

Walther, Donna; 37

Wang, Jia-Bei; 37

Wang, Wen-Fei; 37

Weich, R.; 33
Winger, G.; 105, 117

Woods, James H.; 105, 117

Woolverton, W. L.; 105

Wu, Jun Min; 37

Yu, Lei; 37 



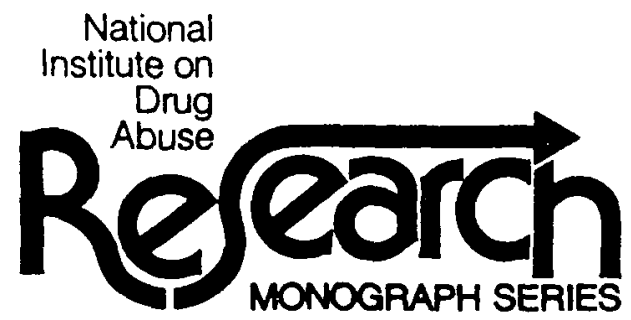

While limited supplies last, single copies of the monographs may be obtained free of charge from the National Clearinghouse for Alcohol and Drug Information (NCADI). Please also contact NCADI for information about availability of coming issues and other publications of the National Institute on Drug Abuse relevant to drug abuse research.

Additional copies may be purchased from the U.S. Government Printing Office (GPO) and/or the National Technical Information Service (NTIS) as indicated. NTIS prices are for paper copy; add $\$ 3.00$ handling charge for each order. Microfiche copies are also available from NTIS. Prices from either source are subject to change.

Addresses are:

\section{NCADI}

National Clearinghouse for Alcohol and Drug Information

P.O. Box 2345

Rockville, MD 20852

(301) 468-2600

(800) 729-6686

GPO

Superintendent of Documents

U.S. Government Printing Office

P.O. Box 371954

Pittsburgh, PA 15220-7954

(202) 738-3238

FAX (202) 512-2233

\section{NTIS}

National Technical Information Service

U.S. Department of Commerce

Springfield, VA 22161

(703) $487-4650$

For information on availability of NIDA Research Monographs from 1975-1993 and those not listed, write to NIDA, Community and Professional Education Branch, Room 10A-39, 5600 Fishers Lane, Rockville, MD 20857. 
26 THE BEHAVIORAL ASPECTS OF SMOKING. Norman A. Krasnegor, Ph.D., ed. (Reprint from 1979 Surgeon General's Report on Smoking and Health.) NCADI \#M26 NTIS PB \#80-118755/AS(A09) \$27.00

42 THE ANALYSIS OF CANNABINOIDS IN BIOLOGICAL FLUIDS. Richard L. Hawks, Ph.D., ed. NCADI \#M42 NTIS PB \#83-136044/AS(A07) \$27.00

50 COCAINE: PHARMACOLOGY, EFFECTS, AND TREATMENT OF ABUSE. John Grabowski, Ph.D., ed. NCADI \#M50

NTIS PB \#85-150381/AS(A07) $\$ 27.00$

52 TESTING DRUGS FOR PHYSICAL DEPENDENCE POTENTIAL AND ABUSE LIABILITY. Joseph V. Brady, Ph.D., and Scott E. Lukas, Ph.D., eds. NCADI \#M52 NTIS PB \#85-150373/AS(A08) \$27.00

53 PHARMACOLOGICAL ADJUNCTS IN SMOKING CESSATION. John Grabowski, Ph.D., and Sharon M. Hall, Ph.D., eds. NCADI \#M53 NTIS PB \#89-123186/AS(A07) \$27.00

54 MECHANISMS OF TOLERANCE AND DEPENDENCE. Charles Wm. Sharp, Ph.D., ed. NCADI \#M54 NTIS PB \#89-103279/AS(A19) $\$ 52.00$

56 ETIOLOGY OF DRUG ABUSE: IMPLICATIONS FOR PREVENTION. CoryI LaRue Jones, Ph.D., and Robert J. Battjes, D.S.W., eds. NCADI \#M56 NTIS PB \#89-123160/AS(A13) \$36.50

61 COCAINE USE IN AMERICA: EPIDEMIOLOGIC AND CLINICAL PERSPECTIVES. Nicholas J. Kozel, M.S., and Edgar H. Adams, M.S., eds. NCADI \#M61 NTIS PB \#89-131866/AS(A11) \$36.50

62 NEUROSCIENCE METHODS IN DRUG ABUSE RESEARCH. Roger M. Brown, Ph.D., and David P. Friedman, Ph.D., eds. NCADI \#M62 NTIS PB \#89-130660/AS(A08) $\$ 27.00$

63 PREVENTION RESEARCH: DETERRING DRUG ABUSE AMONG CHILDREN AND ADOLESCENTS. Catherine S. Bell, M.S., and Robert J. Battjes, D.S.W., eds. NCADI \#M63 NTIS PB \#89-103287/AS(A11) \$36.50

64 PHENCYCLIDINE: AN UPDATE. Doris H. Clouet, Ph.D., ed. NCADI \#M64 NTIS PB \#89-131858/AS(A12) $\$ 36.50$

65 WOMEN AND DRUGS: A NEW ERA FOR RESEARCH. Barbara A. Ray, Ph.D., and Monique C. Braude, Ph.D., eds. NCADI \#M65 NTIS PB \#89-130637/AS(A06) \$27.00

69 OPIOID PEPTIDES: MEDICINAL CHEMISTRY. Rao S. Rapaka, Ph.D.; Gene Barnett, Ph.D.; and Richard L. Hawks, Ph.D., eds. NCADI \#M69" NTIS PB \#89-158422/AS(A17) \$44.50 
70 OPIOID PEPTIDES: MOLECULAR PHARMACOLOGY, BIOSYNTHESIS, AND ANALYSIS. Rao S. Rapaka, Ph.D., and Richard L. Hawks, Ph.D., eds. NCADI \#M70 NTIS PB \#89-158430/AS(A18) \$52.00

72 RELAPSE AND RECOVERY IN DRUG ABUSE. Frank M. Tims, Ph.D., and Carl G. Leukefeld, D.S.W., eds. NCADI \#M72

NTIS P8 \#89-151963/AS(A09) \$36.50

74 NEUROBIOLOGY OF BEHAVIORAL CONTROL IN DRUG ABUSE. Stephen I. Szara, M.D., D.Sc., ed. NCADI \#M74

77 ADOLESCENT DRUG ABUSE: ANALYSES OF TREATMENT RESEARCH. Elizabeth R. Rahdert, Ph.D., and John Grabowski, Ph.D., eds. NCADI \#M77 NTIS PB \#89-125488/AS(A0) $\$ 27.00$

78 THE ROLE OF NEUROPLASTICITY IN THE RESPONSE TO DRUGS. David P. Friedman, Ph.D., and Doris H. Clouet, Ph.D., eds.

NCADI \#M78

NTIS PB \#88-245683/AS(A10) \$36.50

79 STRUCTURE-ACTIVITY RELATIONSHIPS OF THE CANNABINOIDS. Rao S. Rapaka, Ph.D., and

Alexandros Makriyannis, Ph.D., eds.

NCADI \#M79

NTIS PB \#89-109201/AS(A10) $\$ 36.50$

80 NEEDLE SHARING AMONG INTRAVENOUS DRUG ABUSERS: NATIONAL AND INTERNATIONAL PERSPECTIVES. Robert J. Battjes, D.S.W., and Roy W. Pickens, Ph.D., eds.

NCADI \#M80

NTIS PB \#88-236138/AS(A09) $\$ 36.50$

82 OPIOIDS IN THE HIPPOCAMPUS. Jacqueline F. McGinty, Ph.D., and David P. Friedman, Ph.D., eds.

NCADI \#M82

NTIS PB \#88-245691/AS(A06) $\$ 27.00$

83 HEALTH HAZARDS OF NITRITE INHALANTS. Harry W. Haverkos, M.D., and John A. Dougherty, Ph.D., eds. NCADI \#M83

NTIS PB \#89-125496/AS(A06) \$27.00

84 LEARNING FACTORS IN SUBSTANCE ABUSE. Barbara A. Ray, Ph.D., ed. NCADI \#M84 NTIS PB \#89-125504/AS(A10) \$36.50

85 EPIDEMIOLOGY OF INHALANT ABUSE: AN UPDATE. Raquel A. Crider, Ph.D., and Beatrice A. Rouse, Ph.D., eds.

NCADI \#M85

NंTIS PB \#89-123178/AS(A10) $\$ 36.50$

86 COMPULSORY TREATMENT OF DRUG ABUSE: RESEARCH AND CLINICAL PRACTICE. Carl G. Leukefeld, D.S.W., and Frank M. Tims, Ph.D., eds. NCADI \#M86 NTIS PB \#89-151997/AS(A12) \$36.50

87 OPIOID PEPTIDES: AN UPDATE. Rao S. Rapaka, Ph.D., and Bhola N. Dhawan, M.D., eds. NCADI \#M87 
88 MECHANISMS OF COCAINE ABUSE AND TOXICITY. Doris H. Clouet, Ph.D.; Khursheed Asghar, Ph.D.; and Roger M. Brown, Ph.D., eds. NCADI \#M88

NTIS PB \#89-125512/AS(A16) \$44.50

89 BiOlogicAl VULNERABILITY TO DRUG ABUSE. Roy W. Pickens, Ph.D., and Date S. Svikis, B.A., eds.

NCADI \#M89

NTIS PB \#89-125520/AS(A09) $\$ 27.00$

92 TESTING FOR ABUSE LIABILITY OF DRUGS IN HUMANS. Marian W. Fischman, Ph.D., and Nancy K. Mello, Ph.D., eds. NCADI \#M92 NTIS PB \#90-148933/AS(A17) $\$ 44.50$

93 AIDS AND INTRAVENOUS DRUG USE: FUTURE DIRECTIONS FOR COMMUNITY-BASED PREVENTION RESEARCH. C.G. Leukefeld, D.S.W., R.J. Batties, D.S.W., and Zili Amsel, D.S.C., eds. NCADI \#M93 NTIS PB \#90-148933/AS(A14) $\$ 44.50$

94 PHARMACOLOGY AND TOXICOLOGY OF AMPHETAMINE AND RELATED DESIGNER DRUGS. Khursheed Asghar, Ph.D., and Errol De Souza, Ph.D., eds. NCADI \#M94

NTIS PB \#90-148958/AS(A16) $\$ 44.50$

95 PROBLEMS OF DRUG DEPENDENCE, 1989. PROCEEDINGS OF THE 51st ANNUAL SCIENTIFIC MEETING. THE COMMITTEE ON PROBLEMS OF DRUG DEPENDENCE, INC. Louis S. Harris, Ph.D., ed.

NCADI \#M95

NTIS PB \#90-237660/AS(A99) $\$ 67.00$

96 DRUGS OF ABUSE: CHEMISTRY, PHARMACOLOGY, IMMUNOLOGY,

AND AIDS. Phuong Thi Kim Pham, Ph.D., and Kenner Rice, Ph.D., eds.

NCADI \#M96

NTIS PB \#90-237678/AS(A11) \$36.50

97 NEUROBIOLOGY OF DRUG ABUSE: LEARNING AND MEMORY. Lynda Erinoff, Ph.D., ed.

NCADI \#M97

NTIS PB \#90-237686/AS(A11) $\$ 36.50$

98 THE COLLECTION AND INTERPRETATION OF DATA FROM HIDDEN POPULATIONS. Elizabeth Y. Lambert, M.S., ed. NCADI \#M98

NTIS PB \#90-237694/AS(A08) $\$ 27.00$

99 RESEARCH FINDINGS ON SMOKING OF ABUSED SUBSTANCES. C. Nora Chiang, Ph.D., and Richard L. Hawks, Ph.D., eds. NCADI \#M99

NTIS PB \#91-141119(A09) $\$ 27.00$

100 DRUGS IN THE WORKPLACE: RESEARCH AND EVALUATION DATA. VOL II. Steven W. Gust, Ph.D.; J. Michael Walsh, Ph.D.; Linda B.

Thomas, B.S.; and Dennis J. Crouch, M.B.A., eds.

NCADI \#M100

GPO Stock \#017-024-01458-3 \$8.00

101 RESIDUAL EFFECTS OF ABUSED DRUGS ON BEHAVIOR. John W.

Spencer, Ph.D., and John J. Boren, Ph.D., eds.

NCADI \#M101

NTIS PB \#91-172858/AS(A09) $\$ 27.00$ 
103 DRUGS AND VIOLENCE: CAUSES, CORRELATES, AND CONSEQUENCES. Mario De La Rosa, Ph.D., Elizabeth Y. Lambert, M.S., and Bernard Gropper, Ph.D., eds NCADI \#M103

NTIS PB \#91-172841/AS(A13) $\$ 36.50$

104 PSYCHOTHERAPY AND COUNSELING IN THE TREATMENT OF DRUG ABUSE. Lisa Simon Onken, Ph.D., and Jack D. Blaine, M.D., eds. NCADI \#104

NTIS PB \#91-172874/ÄS(A07) $\$ 27.00$

106 IMPROVING DRUG ABUSE TREATMENT. Roy W. Pickens, Ph.D.; Carl G. Leukefeld, D.S.W.; and Charles R. Schuster, Ph.D., eds. NCADI \#M106 NTIS PB \#92-105873(A18) $\$ 50.00$

107 DRUG ABUSE PREVENTION INTERVENTION RESEARCH:

METHODOLOGICAL ISSUES. Carl G. Leukefeld, D.S.W., and William J. Bukoski, Ph.D., eds.

NCADI \#M107

NTIS P8 \#92-160985(A13) $\$ 36.50$

108 CARDIOVASCULAR TOXICITY OF COCAINE: UNDERLYING MECHANISMS. Pushpa V. Thadani, Ph.D., ed. NCADI \#MI 08 NTIS PB \#92-106608(A11) $\$ 36.50$

109 LONGITUDINAL STUDIES OF HIV INFECTION IN INTRAVENOUS DRUG USERS: METHODOLOGICAL ISSUES IN NATURAL HISTORY RESEARCH. Peter Hartsock, Dr.P.H., and Sander G. Genser, M.D., M.P.H., eds. NCADI \#M109 NTIS PB \#92-106616(A08) $\$ 27.00$

111 MOLECULAR APPROACHES TO DRUG ABUSE RESEARCH: RECEPTOR CLONING, NEUROTRANSMITTER EXPRESSION, AND MOLECULAR GENETICS: VOLUME I. Theresa N.H. Lee, Ph.D., ed. NCADI \#M111 NTIS PB \#92-135743(A10) \$36.50

112 EMERGING TECHNOLOGIES AND NEW DIRECTIONS IN DRUG ABUSE RESEARCH. Rao S. Rapaka, Ph.D., Alexandros Makriyannis, Ph.D., and Michael J. Kuhar, Ph.D., eds. NCADI \#M112

NTIS P8 \#92-155449(A15) $\$ 44.50$

113 ECONOMIC COSTS, COST EFFECTIVENESS, FINANCING, AND COMMUNITY-BASED DRUG TREATMENT. William S. Cartwright, Ph.D., and James M. Kaple, Ph.D., eds. NCADI \#M113

NTIS PB \#92-155795(A10) $\$ 36.50$

114 METHODOLOGICAL ISSUES IN CONTROLLED STUDIES ON EFFECTS OF PRENATAL EXPOSURE TO DRUG ABUSE. M. Marlyne Kilbey, Ph.D., and Khursheed Asghar, Ph.D., eds. NCADI \#M114

NTIS PB \#92-146216(A16) $\$ 44.50$

115 METHAMPHETAMINE ABUSE: EPIDEMIOLOGIC ISSUES AND IMPLICATIONS. Marissa A. Miller, D.V.M., M.P.H., and Nicholas J. Kozel, M.S., eds.

NCADI \#M115

NTIS PB \#92-146224/II(A07) $\$ 27.00$ 
116 DRUG DISCRIMINATION: APPLICATIDNS TO DRUG ABUSE RESEARCH. R.A. Glennon, Ph.D., Toubjörn U.C. Järbe, Ph.D., and J. Frankenheim, Ph.D., eds.

NCADI \#M116

NTIS PB \#94-169471(A20) $\$ 52.00$

117 METHODOLOGICAL ISSUES IN EPIDEMIOLOGY, PREVENTION, AND TREATMENT RESEARCH ON DRUG-EXPOSED WOMEN AND THEIR CHILDREN. M. Marlyve Kilbey, Ph.D., and Kursheed Asghar, Ph.D., eds. NCADI \#M117 GPO Stock \#017-024-01472-9 \$12.00 NTIS PB \#93-102101/LL(A18) \$52.00

118 DRUG ABUSE TREATMENT IN PRISONS AND JAILS. Carl G. Leukefeld, D.S.W., and Frank M. Tims, Ph.D., eds.

NCADI \#M118

GPO Stock \#017-024-01473-7 \$16.00 NTIS PB \#93-102143/LL(A14) \$44.50

121 BUPRENORPHINE: AN ALTERNATIVE TREATMENT FOR OPIOID DEPENDENCE. Jack D. Blaine, Ph.D., ed.

NCADI \#M121

GPO Stock \#017-024-01482-6 \$5.00

NTIS PB \#93-129781/LL(A08) $\$ 27.00$

123 ACUTE COCAINE INTOXICATION: CURRENT METHODS OF TREATMENT. Heinz Sorer, Ph.D., ed.

NCADI \#M123

GPO Stock \#017-024-01501-6 \$6.50 NTIS PB \#94-115433/LL(A09) $\$ 27.00$

124 NEUROBIOLOGICAL APPROACHES TO BRAIN-BEHAVIOR INTERACTION. Roger M. Brown, Ph.D., and Joseph Fracella, Ph.D., eds.

NCADI \#M124

GPO Stock \#017-024-01492-3 \$9.00 NTIS PB \#93-203834/LL(A12) \$36.50

125 ACTIVATION OF IMMEDIATE EARLY GENES BY DRUGS OF ABUSE.

Reinhard Grzanna, Ph.D., and Roger M. Brown, Ph.D., eds.

NCADI \#M125

GPO Stock \#017-024-01503-2 \$7.50

NTIS PB \#94-169489(A12) $\$ 36.50$

126 MOLECULAR APPROACHES TO DRUG ABUSE RESEARCH VOLUME II: STRUCTURE, FUNCTION, AND EXPRESSION. Theresa N.H. Lee, Ph.D., ed.

NCADI \#M126

NTIS PB \#94-169497(A08) $\$ 27.00$

127 PROGRESS AND ISSUES IN CASE MANAGEMENT.

Rebecca Sager Ashery, D.S.W., ed.

NCADI \#MI 27

NTIS PB \#94-169505(A18) $\$ 52.00$

128 STATISTICAL ISSUES IN CLINICAL TRIALS FOR TREATMENT OF OPIATE DEPENDENCE. Ram B. Jain, Ph.D., ed. NCADI \#M128

NTIS PB \#93-203826/LL(A09) \$27.00

129 INHALANT ABUSE: A VOLATILE RESEARCH AGENDA. Charles W. Sharp, Ph.D.; Fred Beauvais, Ph.D., and Richard Spence, Ph.D., eds.

NCADI \#M129 
130 DRUG ABUSE AMONG MINORITY YOUTH: ADVANCES IN RESEARCH AND METHODOLOGY. Mario De La Rosa, Ph.D., and Juan-Luis Recio Adrados, Ph.D., eds.

NCADI \#M130

GPO Stock \#017-024-01506-7 \$14:00 NTIS PB \#94-169513 (A15) $\$ 44.50$

131 IMPACT OF PRESCRIPTION DRUG DIVERSION CONTROL SYSTEMS ON MEDICAL PRACTICE AND PATIENT CARE. James R. Cooper, Ph.D.; Dorynne J. Czechowicz, M.D., Stephen P. Molinari, J.D., R.Ph., and Robert C. Peterson, Ph.D., eds.

NCADI \#M131

GPO Stock \#017-024-01505-9 \$14.00 NTIS PB \#94-169521(A15) \$44.50

134 MEDICATIONS DEVELOPMENT: DRUG DISCOVERY, DATABASES, AND COMPUTER-AIDED DRUG DESIGN. Rao S. Rapaka, Ph.D., and Richard L. Hawks, Ph.D., eds.

NCADI \#M134

GPO Stock \#017-024-01511-3 \$11.00 NTIS PB \#94-169547(A14) $\$ 44.50$

135 COCAINE TREATMENT: RESEARCH AND CLINICAL PERSPECTIVES. Frank M. Tims, Ph.D., and Carl G. Leukefeld, D.S.W., eds.

NCADI \#M135

GPO Stock \#017-024-01520-2 \$11.00 NTIS PB \#94-169554(A13) \$36.50

136 ASSESSING NEUROTOXICITY OF DRUGS OF ABUSE. Lynda Erinoff, Ph.D., ed.

NCADI \#M136

GPO Stock \#017-024-01518-1 \$11.00 NTIS PB \#94-169562(A13) \$36.50

137 BEHAVIORAL TREATMENTS FOR DRUG ABUSE AND DEPENDENCE. Lisa Simon Onken, Ph.D.; Jack D. Blaine, M.D., and John J. Boren, Ph.D., eds. NCADI \#M137 GPO Stock \#017-024-01519-9 \$13.00 NTIS PB \#94-169570(A15) $\$ 44.50$

138 IMAGING TECHNIQUES IN MEDICATIONS DEVELOPMENT: CLINICAL AND PRECLINICAL ASPECTS. Heinz Sorer, Ph.D., and Rao S. Rapaka, Ph.D., eds.

NCADI \#M138

139 SCIENTIFIC METHODS FOR PREVENTION INTERVENTION RESEARCH. Arturo Cazares, M.D., M.P.H., and Lula A. Beatty, Ph.D., eds.

NCADI \#M139

140 PROBLEMS OF DRUG DEPENDENCE, 1993: PROCEEDINGS OF THE 55TH ANNUAL SCIENTIFIC MEETING, THE COLLEGE ON PROBLEMS OF DRUG DEPENDENCE, INC. VOLUME I: PLENARY SESSION SYMPOSIA AND ANNUAL REPORTS. Louis S. Harris, Ph.D., ed.

NCADI \#M140

141 PROBLEMS OF DRUG DEPENDENCE, 1993: PROCEEDINGS OF THE 55TH ANNUAL SCIENTIFIC MEETING, THE COLLEGE ON PROBLEMS OF DRUG 
DEPENDENCE, INC. VOLUME II: ABSTRACTS. Louis S. Harris, Ph.D., ed. NCADI \#M141

142 ADVANCES IN DATA ANALYSIS FOR PREVENTION INTERVENTION RESEARCH. Linda M. Collins, Ph.D., and Larry A. Seitz, Ph.D., eds.

NCADI \#M142

143 THE CONTEXT OF HIV RISK AMONG DRUG USERS AND THEIR SEXUAL PARTNERS. Robert J. Battjes, D.S.W., Zili Sloboda, Sc.D., and William C. Grace, Ph.D., eds.

NCADI \#M143

144 THERAPEUTIC COMMUNITY: ADVANCES IN RESEARCH AND APPLICATION. Frank M. Tims, Ph.D., George De Leon, Ph.D., and Nancy Jainchill, Ph.D., eds.

NCADI \#M144

145 NEUROBIOLOGICAL MODELS FOR EVALUATING MECHANISMS UNDERLYING COCAINE ADDICTION. Lynda Erinoff, Ph.D., and Roger M. Brown, Ph.D., eds.

NCADI \#M145

146 HALLUCINOGENS: AN UPDATE. Geraline C. Lin, Ph.D., and Richard A. Glennon, Ph.D., eds.

NCADI \#M146

147 DISCOVERY OF NOVEL OPIOID MEDICATIONS. Rao S. Rapaka, Ph.D., and Heinz Sorer, Ph.D., eds.

NCADI \#M147

148 EPIDEMIOLOGY OF INHALANT ABUSE: AN INTERNATIONAL

PERSPECTIVE. Nicholas J. Kozel, M.S., Zili Sloboda, Sc.D., and Mario R. De La Rosa, Ph.D., eds.

NCADI \#M148

149 MEDICATIONS DEVELOPMENT FOR THE TREATMENT OF PREGNANT ADDICTS AND THEIR INFANTS. C. Nora Chiang, Ph.D., and Loretta P. Finnegan, M.D., eds.

NCADI \#M149

150 INTEGRATING BEHAVIORAL THERAPIES WITH MEDICATIONS IN THE TREATMENT OF DRUG DEPENDENCE. Lisa Simon Onken, Ph.D., Jack D. Blaine, M.D., and John J. Boren, Ph.D., eds.

NCADI \#M150 
151 SOCIAL NETWORKS, DRUG ABUSE, AND HIV TRANSMISSION. Richard H. Needle, Ph.D., M.P.H., Susan L. Coyle, M.A., Sander G. Genser, M.D., M.P.H., and Robert T. Trotter II, Ph.D., eds.

NCADI \#M151 


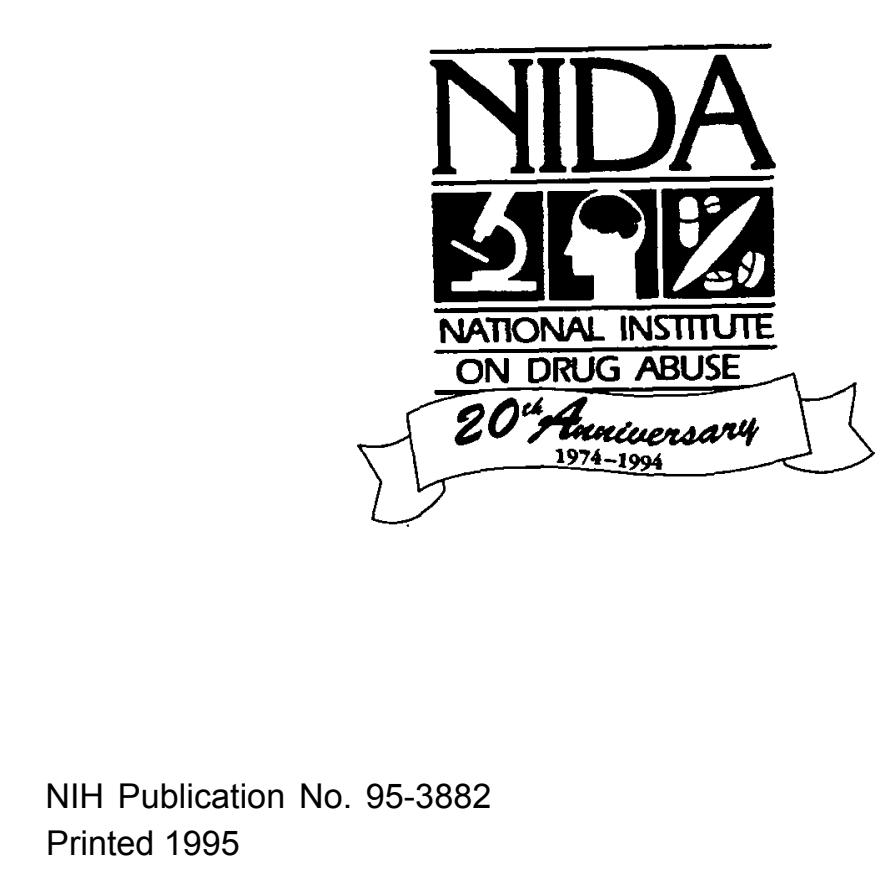

